\title{
Diatom diversity in the lakes of the Pyrenees: an iconographic reference
}

\author{
Carlos A. Rivera-Rondón ${ }^{1, *}$ and Jordi Catalan ${ }^{2}$ \\ ${ }^{1}$ UNESIS, Departamento de Biología, Pontificia Universidad Javeriana, Cra. 7. 40-82, Bogotá, Colombia. \\ ${ }^{2}$ CREAF- CSIC, Edifici C, Campus UAB, Cerdanyola del Vallès, E-08193, Spain.
}

* Corresponding author: crivera@javeriana.edu.co

Received: $15 / 07 / 16$

Accepted: 20/02/17

\begin{abstract}
Diatom diversity in the lakes of the Pyrenees: an iconographic reference

Environmental assessments and palaeoreconstructions can take advantage of the high diatom diversity present in mountain lakes. There are only a few studies dealing with the diatom flora of the Pyrenees and research on species taxonomy, distribution and ecology are still at an initial stage. With the aim of updating the list of species present and providing iconographic information for harmonising past and future studies, we report 549 taxa from a survey of 83 lakes across the geographical extent of the Pyrenean lakes comprising a broad range of environmental conditions and in the sedimentary record of the Burg lake. This study is a first step in the development of a comprehensive on-line atlas of Pyrenean diatoms including species occurrence, distribution and ecology (www.diatopyr.com). Samples were collected both from littoral epilithic substrates and the top of the sediment in the deepest zone of the lake. Additionally, we include also taxa that were present in the Late Glacial and Holocene sedimentary records of the Burg palaeolake, which were not found in the survey. The genera including more diversity were Pinnularia, Gomphonema, Eunotia and Nitzschia. About 25\% of taxa remain to be determined at fine taxonomic resolution, many of them are likely to be species or varieties not described yet.
\end{abstract}

Key words: Diatom diversity, epilithon, oligotrophic lakes, mountain lakes.

\section{RESUMEN}

\section{La diversidad de diatomeas en los lagos de los Pirineos: Una referencia iconográfica}

La gran diversidad de diatomeas presentes en los lagos de montaña puede ser utilizada para el desarrollo de evaluaciones y paleoreconstrucciones ambientales. Hay pocos estudios sobre la flora de diatomeas de los Pirineos y los trabajos sobre su taxonomía, distribución y ecología están todavía en una fase inicial. Con el objetivo de actualizar la lista de especies registradas en los Pirineos y facilitar la armonización taxonómica de los trabajos pasados y futuros sobre diatomeas, se presenta un trabajo iconográfico que incluye 549 taxones encontrados en 83 lagos estudiados a lo largo de amplio rango de condiciones ambientales y en el registro sedimentario del lago Burg. Este es un primer paso para el desarrollo de un extenso atlas en Internet de las diatomeas de los Pirineos que incluya la ocurrencia, distribución y ecología de las especies (www.diatopyr.com). Las muestras se colectaron en el epiliton de la zona litoral y en la superficie de los sedimentos de la zona más profunda del lago. Adicionalmente, se incluyen taxones encontrados en el registro sedimentario del Tardiglacial y Holoceno del paleolago de Burg. Los géneros que tuvieron mayor diversidad fueron Pinnularia, Gomphonema, Eunotia y Nitzschia. Alrededor del $25 \%$ de los taxones encontrados no se identificaron con una resolución taxonómica a nivel de especie, probablemente muchos de ellos corresponden a identidades taxonómicas no descritas todavía.

Palabras clave: Diversidad de diatomeas, epiliton, lagos oligotróficos, lagos de montaña. 


\section{INTRODUCTION}

Diatom diversity is particularly high in oligotrophic environments including mountain lakes similar as those in the Pyrenees (Lange-Bertalot \& Metzeltin, 1996). Of the 1088 taxa recorded for the Iberian Peninsula, approximately 300 are registered in the Spanish Pyrenean area and Andorra (Aboal et al., 2003). Likely these taxa number is still a high under-estimation of the diatom diversity in the Pyrenees as monographic studies on diatoms are limited and outdated (Hustedt, 1939; Carter, 1970). The presence of taxa not yet described is also likely. The progression in the description of new species in European freshwater ecosystems points to a large unknown diversity in remote and oligotrophic environments. Even for the Central European freshwater ecosystems that have been studied extensively, recent taxonomical investigations continue describing new taxa (Krammer \& Lange-Bertalot, 1991b; Lange-Bertalot, 1993, 2001; Lange-Bertalot \& Metzeltin, 1996; Krammer, 1997a, 1997b, 2002, 2003; Reichardt, 1999; Lange-Bertalot et al., 2003; Werum \& Lange-Bertalot, 2004; Levkov, 2009; Lange-Bertalot et al., 2011). On the other hand, beyond biodiversity studies, diatoms are used in many applications, from ecological assessments (Denicola et al., 2004) to environmental reconstructions (Quillen et al., 2013). This has also been the case in the Pyrenean lakes; diatoms are included in the protocols for the assessment and monitoring of the ecological status of lakes (Clarke et al.,

Table 1. Geographic, morphological, lithological, land cover and chemical characteristic of the lakes studied. Chemical data were obtained in a unique sampling performed from 9/7/2000 to 23/8/2000 (Catalan et al., 2009a). Características geográficas, morfológicas, litológicas, cobertura de la cuenca y descripción general química de los lagos estudiados. Los datos químicos provienen de un único muestreo realizado entre 9/7/2000 to 23/8/2000 (Catalan et al., 2009a).

\begin{tabular}{|c|c|}
\hline Variable & Median (range) \\
\hline Altitude (m.a.s.1.) & $2305(1620-2990)$ \\
\hline Lake area (ha) & $5.5(0.2-53.2)$ \\
\hline Catchment area (ha) & $114.6(7-5437.9)$ \\
\hline Maximum depth (m) & $17(0.7-123)$ \\
\hline Relative depth $(\%)$ & $6.5(0.8-18)$ \\
\hline Ice-cover duration (days) & $185(115-215)$ \\
\hline \multicolumn{2}{|l|}{ Lithology (>30\% lake catchment area) } \\
\hline Metamorphic $(\%)$ & $27.1(0-100)$ \\
\hline Plutonic $(\%)$ & $48.2(0-100)$ \\
\hline Detrital $(\%)$ & $17.7(0-100)$ \\
\hline Carbonate $(\%)$ & $17.5(0-90)$ \\
\hline \multicolumn{2}{|l|}{ Land cover } \\
\hline Glacier presence $(\%)$ & $0(0-15)$ \\
\hline Bare rock $(\%)$ & $30(0-90)$ \\
\hline Meadows $(\%)$ & $15(0-90)$ \\
\hline Shrubs $(\%)$ & $0(0-60)$ \\
\hline Coniferous $(\%)$ & $0(0-40)$ \\
\hline Irradiance at lake bottom $(\%)$ & $6.6(0-88.8)$ \\
\hline Summer surface temperature $\left({ }^{\circ} \mathrm{C}\right)$ & $12.6(3.8-18.5)$ \\
\hline $\mathrm{pH}$ & $7.0(4.5-9.0)$ \\
\hline Acid Neutralizing Capacity ( $\mu \mathrm{eq} / \mathrm{L})$ & $123(0-1696)$ \\
\hline $\mathrm{SO}_{4}^{2-}(\mu \mathrm{eq} / \mathrm{L})$ & $41.3(10.3-1240.0)$ \\
\hline $\mathrm{Ca}^{2+}(\mu \mathrm{eq} / \mathrm{L})$ & $139.5(20.0-1195.0)$ \\
\hline $\mathrm{Mg}^{2+}(\mu \mathrm{eq} / \mathrm{L})$ & $14.6(4.0-557.3)$ \\
\hline $\mathrm{TP}(\mu \mathrm{g} / \mathrm{L})$ & $3.4(0.94-33.3)$ \\
\hline $\mathrm{TN}(\mu \mathrm{g} / \mathrm{L})$ & $177.1(43.9-967.9)$ \\
\hline $\mathrm{NO}_{3}^{-}(\mu \mathrm{eq} / \mathrm{L})$ & $5.1(0-19.9)$ \\
\hline
\end{tabular}


2005; Catalan et al., 2006) and have been used in reconstructing $\mathrm{pH}$ and alkalinity in paleolimnological studies (Catalan et al., 2009b; Catalan et al., 2014). Any consistent application of diatoms as indicators requires as much taxonomic quality as accuracy in the measurement of environmental variables and adequate statistical procedures (Birks, 1994). Although discussions about the latter aspect are common (Anderson, 2000; Juggins, 2013), there is no statistical improvement able to deal with the lack of harmonised and precise taxonomy. For instance, the higher the taxonomical resolution, the better is the performance of models used to reconstruct nutrients and major-ion content (Rimet \& Bouchez, 2012). Beyond the need to pursuit the best and most updated taxonomy, ecological applications will benefit from rich iconographical information facilitating the harmonisation among current studies and those of the past under a long-term situation of periodic taxonomic revisions.

\section{METHODS}

The study of the diatom species distribution was based on a survey of 83 lakes of the Pyrenees performed from 9/7/2000 to 23/8/2000 (Catalan et al., 2009a). Lakes were distributed across a wide range of environmental conditions determined by bedrock, altitude and lake size changes (Table 1, Table 2, Fig. 1). The lakes were selected to achieve a stratified representation of the environmental variability and covering the geographical extremes. The taxonomical study was complemented with the taxa in the diatom record of the Burg palaeolake (Pèlachs et al., 2011), including a Late Glacial and early Holocene sedimentary sequence.

Two types of samples were collected at each lake in the regional study: top sediment and epilithon. Top sediment samples were from the deepest part of the lake, collected using a gravity corer and slicing the upper $0.5 \mathrm{~cm}$. Epilithon sam-

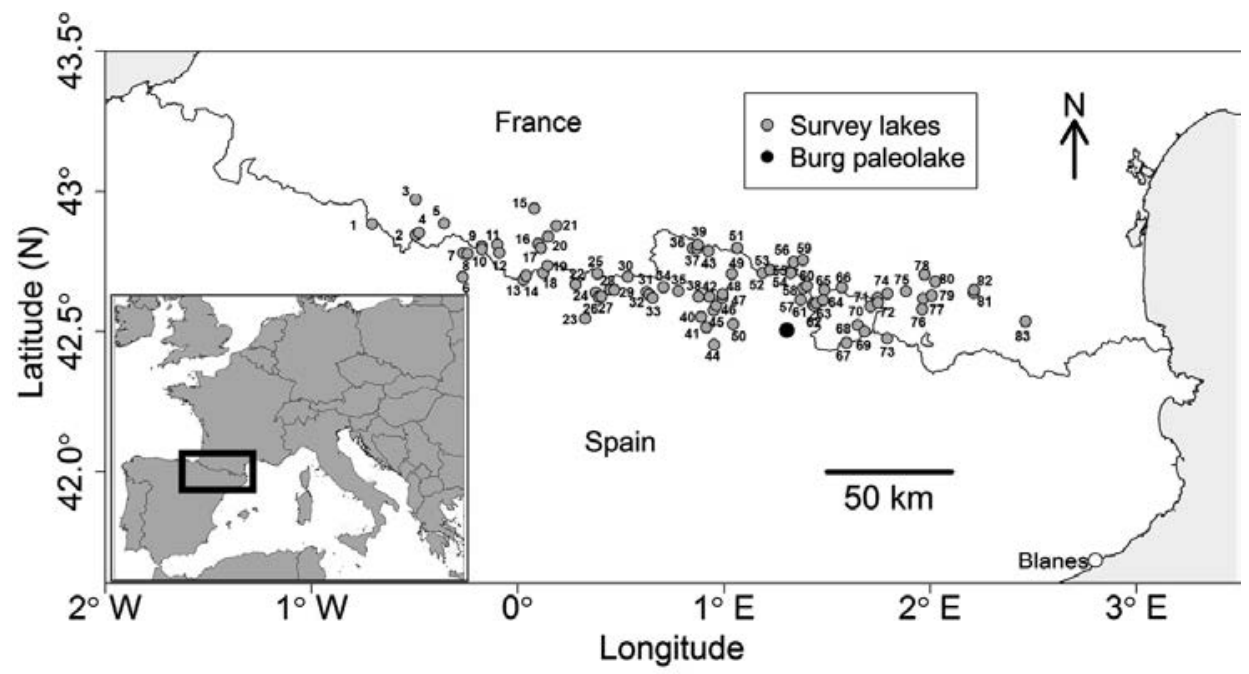

Figure 1. Map showing the location of the lakes included in the survey and Burg palaeolake (black point). Acherito (1), Bersau (2), Montagnon (3), Roumassot (4), Ormiélas (5), Asnos (6), Pondiellos sup. (7), Arnales (8), Arratille (9), Col d'Arratille (10), Estom (11), Glacé (12), Helado del Monte Perdido (13), Helado de Marboré (14), Bleu (15), Tourrat (16), Cap Long (17), La Munia Sup. (18), Barroude Inf. (19), Les Laquettes 1 (20), Port Bielh (21), Urdiceto (22), Basa de la Mora (23), Pixón (24), Bachimala Sup. (25), Sen (26), Chelau Sup. (27), Posets (28), Eriste (29), Lliterola (30), Cregüeña (31), Coronas (32), Llosás (33), Puis (34), Redon (35), Nere de Güèrri (36), Pica Palòmera (37), Monges (38), Long de Liat (39), Llebreta (40), Gran del Pessó (41), Plan (42), Montoliu (43), Filià (44), Llong (45), Gelat Bergús (46), Illa (47), Gerber (48), Airoto (49), Gran de Mainera (50), Rond (51), Inferior de la Gallina (52), Mariola (53), Senó (54), Romedo de Dalt (55), Aubé (56), Aixeus (57), Sotllo (58), Garbet (59), Pica (60), Baiau Superior (61), Negre (62), Forcat Inf. (63), Angonella de Més Amunt (64), Més amunt de Tristaina (65), Blaou (66), Gran de la Pera (67), Ensangents Sup. (68), Montmalús (69), Canals Roges (70), Albe (71) Siscar (72), Malniu (73), Compte (74), Aygue (75), Longue, Trebens (76), Blau (77), Bleu de Rabassoles (78), Gros de Camporrells (79), Laurenti (80), Negre (81), Estelat (82), L'Estagnol (83). Mapa en el que se presenta la ubicación de los lagos muestreados y el paleolago Burg. 
Table 2. List of lakes studied. ID corresponds to the number of each lake in Figure 1. Lista de los lagos estudiados. El número en paréntesis corresponde con el número de cada lago en la figura 1.

\begin{tabular}{|c|c|c|c|c|}
\hline ID & Lake & Latitude $\left({ }^{\circ} \mathrm{N}\right)$ & Longitude $\left({ }^{\circ} \mathrm{E}\right)$ & Altitude (m a.s.l.) \\
\hline 1 & Acherito & 42.88089 & -0.70608 & 1875 \\
\hline 2 & Bersau & 42.84062 & -0.49454 & 2077 \\
\hline 3 & Montagnon & 42.96846 & -0.49387 & 2003 \\
\hline 4 & Roumassot & 42.84925 & -0.47815 & 1845 \\
\hline 5 & Ormiélas & 42.8838 & -0.35562 & 1974 \\
\hline 6 & Asnos & 42.6918 & -0.26629 & 2060 \\
\hline 7 & Pondiellos sup. & 42.77699 & -0.26312 & 2745 \\
\hline 8 & Arnales & 42.77503 & -0.24231 & 2305 \\
\hline 9 & Arratille & 42.80158 & -0.17364 & 2247 \\
\hline 10 & Col d'Arratille & 42.79104 & -0.17274 & 2501 \\
\hline 11 & Estom & 42.80752 & -0.09853 & 1804 \\
\hline 12 & Glacé & 42.7783 & -0.08845 & 2571 \\
\hline 13 & Helado del Monte Perdido & 42.68213 & 0.02771 & 2990 \\
\hline 14 & Helado de Marboré & 42.69659 & 0.04104 & 2592 \\
\hline 15 & Bleu & 42.93705 & 0.08174 & 1950 \\
\hline 16 & Tourrat & 42.80998 & 0.09966 & 2636 \\
\hline 17 & Cap Long & 42.7951 & 0.11305 & 2845 \\
\hline 18 & La Munia Sup. & 42.70615 & 0.12499 & 2537 \\
\hline 19 & Barroude Inf. & 42.73264 & 0.14478 & 2377 \\
\hline 20 & Les Laquettes 1 & 42.83592 & 0.14806 & 2085 \\
\hline 21 & Port Bielh & 42.87417 & 0.18846 & 2290 \\
\hline 22 & Urdiceto & 42.66672 & 0.2816 & 2378 \\
\hline 23 & Basa de la Mora & 42.54526 & 0.32771 & 1908 \\
\hline 24 & Pixón & 42.63682 & 0.37986 & 2199 \\
\hline 25 & Bachimala Sup. & 42.7044 & 0.38761 & 2630 \\
\hline 26 & Sen & 42.62148 & 0.39312 & 2360 \\
\hline 27 & Chelau Sup. & 42.62419 & 0.40667 & 2805 \\
\hline 28 & Posets & 42.64681 & 0.4494 & 2550 \\
\hline 29 & Eriste & 42.64646 & 0.46808 & 2411 \\
\hline 30 & Lliterola & 42.69367 & 0.5338 & 2734 \\
\hline 31 & Cregüeña & 42.63867 & 0.6253 & 2640 \\
\hline 32 & Coronas & 42.62997 & 0.63848 & 2740 \\
\hline 33 & Llosás & 42.61766 & 0.65483 & 2480 \\
\hline 34 & Puis & 42.65542 & 0.7076 & 2056 \\
\hline 35 & Redon & 42.64208 & 0.77951 & 2235 \\
\hline 36 & Nere de Güèrri & 42.79334 & 0.85029 & 2280 \\
\hline 37 & Pica Palòmera & 42.79377 & 0.86878 & 2308 \\
\hline 38 & Monges & 42.62301 & 0.87701 & 2418 \\
\hline 39 & Long de Liat & 42.80655 & 0.87398 & 2140 \\
\hline 40 & Llebreta & 42.55083 & 0.89031 & 1620 \\
\hline 41 & Gran del Pessó & 42.51264 & 0.91563 & 2493 \\
\hline 42 & Plan & 42.62248 & 0.9307 & 2188 \\
\hline
\end{tabular}


Table 2. (cont.) List of lakes studied. ID corresponds to the number of each lake in Figure 1. Lista de los lagos estudiados. El número en paréntesis corresponde con el número de cada lago en la figura 1.

\begin{tabular}{|c|c|c|c|c|}
\hline ID & Lake & Latitude $\left({ }^{\circ} \mathrm{N}\right)$ & Longitude $\left({ }^{\circ} \mathrm{E}\right)$ & Altitude (m a.s.l.) \\
\hline 43 & Montoliu & 42.78467 & 0.92614 & 2375 \\
\hline 44 & Filià & 42.45122 & 0.95328 & 2140 \\
\hline 45 & Llong & 42.57431 & 0.95063 & 2000 \\
\hline 46 & Gelat Bergús & 42.59106 & 0.96331 & 2493 \\
\hline 47 & Illa & 42.61836 & 0.99348 & 2452 \\
\hline 48 & Gerber & 42.63065 & 0.99471 & 2170 \\
\hline 49 & Airoto & 42.70281 & 1.03922 & 2210 \\
\hline 50 & Gran de Mainera & 42.52516 & 1.04585 & 2450 \\
\hline 51 & Rond & 42.7944 & 1.0645 & 1929 \\
\hline 52 & Inferior de la Gallina & 42.70618 & 1.18763 & 2270 \\
\hline 53 & Mariola & 42.71737 & 1.22434 & 2276 \\
\hline 54 & Senó & 42.71203 & 1.32291 & 2130 \\
\hline 55 & Romedo de Dalt & 42.70601 & 1.32465 & 2110 \\
\hline 56 & Aubé & 42.74549 & 1.33801 & 2094 \\
\hline 57 & Aixeus & 42.61098 & 1.3718 & 2370 \\
\hline 58 & Sotllo & 42.652 & 1.38445 & 2346 \\
\hline 59 & Garbet & 42.7526 & 1.38299 & 1683 \\
\hline 60 & Pica & 42.66079 & 1.4024 & 2880 \\
\hline 61 & Baiau Superior & 42.59627 & 1.43188 & 2480 \\
\hline 62 & Negre & 42.58913 & 1.43826 & 2627 \\
\hline 63 & Forcat Inf. & 42.60074 & 1.44883 & 2631 \\
\hline 64 & Angonella de Mes Amunt & 42.61015 & 1.48138 & 2440 \\
\hline 65 & Mes amunt de Tristaina & 42.64685 & 1.48741 & 2300 \\
\hline 66 & Blaou & 42.655 & 1.57264 & 2350 \\
\hline 67 & Gran de la Pera & 42.45818 & 1.59509 & 2350 \\
\hline 68 & Ensangents Sup. & 42.52134 & 1.64923 & 2550 \\
\hline 69 & Montmalús & 42.49832 & 1.68263 & 2440 \\
\hline 70 & Canals Roges & 42.58673 & 1.7118 & 2410 \\
\hline 71 & Albe & 42.61835 & 1.74514 & 2355 \\
\hline 72 & Siscar & 42.6014 & 1.74718 & 2187 \\
\hline 73 & Malniu & 42.47378 & 1.79238 & 2250 \\
\hline 74 & Compte & 42.63366 & 1.79306 & 1726 \\
\hline 75 & Aygue Longue & 42.64189 & 1.88263 & 2076 \\
\hline 76 & Trebens & 42.5778 & 1.96255 & 2306 \\
\hline 77 & Blau & 42.61554 & 1.96708 & 2531 \\
\hline 78 & Bleu de Rabassoles & 42.70038 & 1.97274 & 1920 \\
\hline 79 & Gros de Camporrells & 42.62583 & 2.00788 & 2255 \\
\hline 80 & Laurenti & 42.67525 & 2.02582 & 1936 \\
\hline 81 & Negre & 42.63592 & 2.21141 & 2083 \\
\hline 82 & Estelat & 42.64632 & 2.21351 & 2021 \\
\hline \multirow[t]{2}{*}{83} & L'Estagnol & 42.53361 & 2.46276 & 2164 \\
\hline & Burg Palaeolake & 42.503929 & 1.304633 & 1821 \\
\hline
\end{tabular}


ples were obtained by brushing five stones in the shoreline area. Stones were selected in areas between 0.3 and $1.0 \mathrm{~m}$ depth. The number of samples studied for sediment and epilithon was smaller than 83 provided that in some lakes was not possible to collect both or there were not diatoms in the samples.

The total number of samples processed were 778 (76 top sediment; 78 epilithon, and 624 Burg Lake core). Formaldehyde was removed from the lake survey samples before further processing. Burg Lake samples were placed directly in borosilicate tubes and rehydrated with one millilitre of Milli-Q water. Samples were digested following an oxidative procedure that started by adding $0.3 \mathrm{ml} 1 \mathrm{~N} \mathrm{HCl}$ and $5 \mathrm{ml} 30 \%$ $\mathrm{H}_{2} \mathrm{O}_{2}$. The oxidation was initially performed in a water bath at room temperature to avoid out of control exothermic reactions in samples with high metal content. The temperature of the bath was gradually increased to $70-80{ }^{\circ} \mathrm{C}$, and the level of $\mathrm{H}_{2} \mathrm{O}_{2}$ was maintained until all the organic material was removed. To open the diatoms valves and make transparent some non-digested material, $1 \mathrm{ml} 1 \mathrm{~N} \mathrm{HCl}$ was added. After digestion, residual dissolved chemicals were removed by washing until the sample $\mathrm{pH}$ reached 6 .

Once the samples were cleaned, samples were kept in an ultrasonic bath during five minutes to disperse the valves. After that, the diatom suspension was diluted to obtain a suitable solution that was carefully dropped on a round glass coverslip. A large drop was added to assured that the coverslip margin was reached. The coverslips were kept undisturbed in dust-free conditions until they were completely dry. Finally, the samples were mounted in Naphrax (refractive index $=1.74$ ).

Diatom taxonomic determination was made using a Zeiss Axio Imager A.1 differential interference contrast microscope with a plan-apochromatic $100 \times$ objective. A minimum of 1000 and 500 valves was counted from the survey lakes and Burg Lake samples, respectively. Diagnostic morphological traits were studied using a field emission scanning electron microscope Hitachi S-4100-FE. The determination was based firstly on general taxonomical studies of the diatoms of the Pyrenees (Hustedt, 1939; Carter, 1970) and European freshwater ecosystems (Krammer \& Lange-Bertalot, 1986, 1988, 1991a, 1991b; Lange-Bertalot \& Metzeltin, 1996; Krammer, 2000, 2002, 2003; Lange-Bertalot, 2001; LangeBertalot et al., 2003; Krammer \& Lange-Bertalot, 2004; Werum \& Lange-Bertalot, 2004a; Levkov, 2009; Hofmann et al., 2011; LangeBertalot et al., 2011), and, secondly, on a large number of papers and books on specific taxonomical updates and regional iconographies (Lange-Bertalot \& Krammer, 1987, 1989; Reichardt \& Lange-Bertalot, 1991; Lange-Bertalot, 1993, 1997; Bukhtiyarova \& Round, 1996; Krammer, 1997, 1997b; Reichardt, 1997, 1999, 2007; Håkansson, 2002; Houk, 2003; Houk \& Klee, 2004; Van De Vijver et al., 2004; Houk et al., 2010).

Specimens that resembled a known species but showed differences in diagnostic traits or requiring additional comparisons were distinguished as confer ("cf."). Specimens with traits markedly different from the most similar species were operatively named using a combination of the name of the lake where they were found the first time and consecutive numbers, if necessary. They are likely new species or varieties.

Richness estimators, such as Chao, Jackknife, and Bootstrapping (Colwell \& Coddington, 1995) were performed using $\mathrm{R}$ language and the package vegan 2.4-0 (Oksanen et al., 2012; $\mathrm{R}$ Core Team 2015).

\section{RESULTS AND DISCUSSION}

\section{General description of the diatom flora}

The diatom taxa found in this study are listed in the iconographic catalogue. A total number of 73 genera were found in the lakes studied. The genera with more species were Pinnularia (40 taxa), Gomphonema (39), Eunotia (35), Nitzschia (32), Navicula (27), Naviculadicta (24), Encyonema (22) and Fragilaria (19). Considering the survey lakes exclusively, the ranking was similar: $E u$ notia (34 taxa), Gomphonema (31), Pinnularia (31), Nitzschia (30), Navicula (23), Naviculadicta (22), Encyonema (20) and Fragilaria (18). 


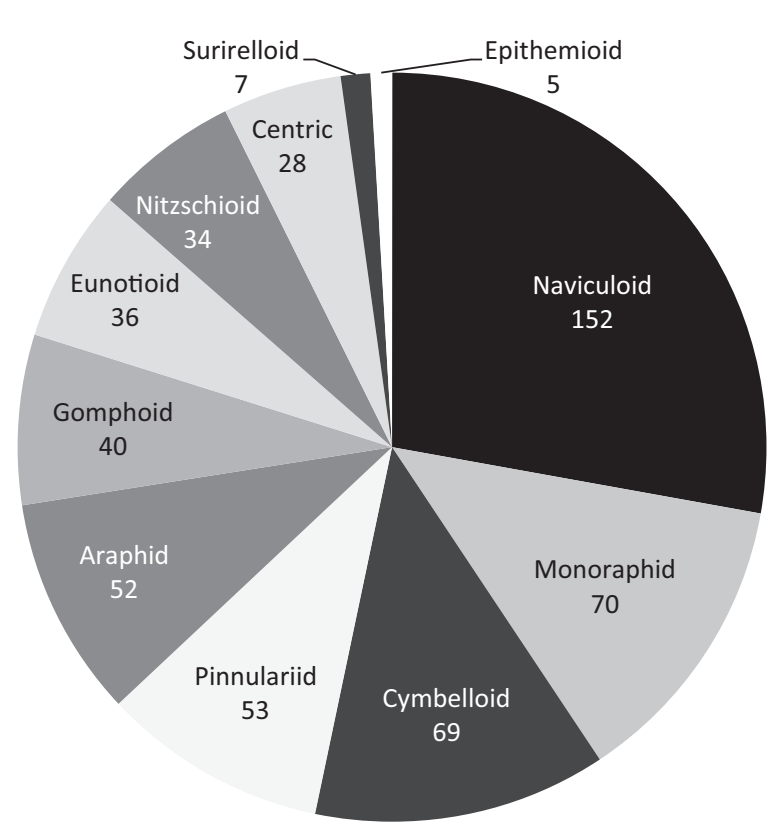

Figure 2. Taxa distribution into morphological types. Distribución de los taxones de acuerdo con su tipo morfológico.

Considering artificial morphological types, not strictly evolutionary (Fig. 2), the highest number of taxa were in Naviculoid, Monoraphid and Cymbelloid forms, respectively. The types with the lowest number were Epithemioid, Surirelloid and Centric. This general pattern was replicated in the epilithon, sediment and Burg Lake sample sets.

The total taxa (species and infraspecies taxa) distinguished were 549: 477 in the lake survey and 244 in the Burg Lake (Fig. 3). In the lake survey, the sediment samples showed a higher number of taxa (417) than the epilithon samples (355). 89 and 42 taxa were exclusive of the sediment and epilithon samples, respectively.

A $7.6 \%$ of taxa were exclusive of epilithon samples, among them: Chamaepinnularia No. 1 Negre, Eunotia tenella (Grunow) Hustedt, Sellaphora stroemii (Hustedt) Kobayasi, Fragilaria sp. No. 6 Blaou, Pinnularia cf. kuetzingii Krammer, Cymbella neoleptoceros var. tenuistriata Krammer, Gomphoneis cf. olivaceoides (Hustedt) Carter. A $16 \%$ of taxa were only found in the sediments, among them: Sellaphora pseudopupula (Krasske) Lange-Bertalot, Fragilaria

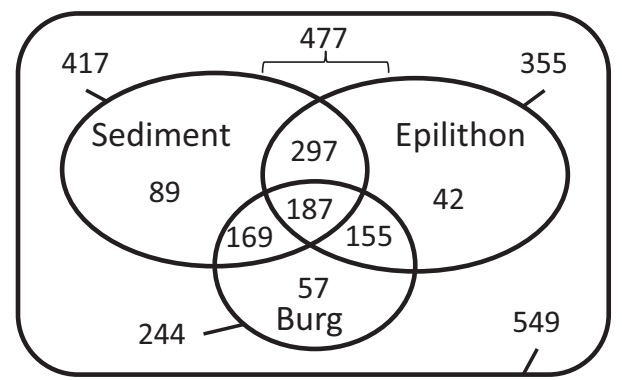

Figure 3. Total, coincident and exclusive taxa found in the lake survey set (sediment and epilithon) and Burg palaeolake samples. Taxones totales, coincidentes y exclusivos encontrados en el muestreo regional (sedimento y epiliton) y en el paleolago de Burg.

sp. No. 1 Airoto, Navicula venerablis Hohn \& Hellerman, Navicula sp. No. 3 Laurenti, Encyonema sp. No. 1 Mora, Navicula cryptocephala Kützing, Pinnularia cf. rumrichae Krammer, Geissleria similis (Krasske) Lange-Bertalot \& Metzeltin, Cymbopleura subaequalis (Grunow) Krammer, Navicula trophicatrix Lange-Bertalot, Surirella angusta Kützing, Meridion circulare var. constrictum (Ralfs) Van Heurck and Stauroneis gracilis Ehrenberg.

There were many taxa more frequent in the epilithon samples than in the sediments; the most common were: Encyonopsis cf. krammeri Reichardt, Navicula notha Wallace, Pinnularia subinterrupta Krammer \& Schroeter, Nitzschia cf. alpina Hustedt, Encyonopsis minuta Krammer et Reichardt, Eunotia glacialis Meister, Encyonema reichardtii (Krammer) Mann, Cymbella cf. neocistula Krammer, Cymbella cf. cymbiformis Agardh, Eunotia novaisiae var. altopyrenaica Lange-Bertalot \& Rivera-Rondón, Gomphonema capitatum Ehrenberg, Delicata delicatula (Kützing) Krammer, Reimeria sinuata (Gregory) Kociolek \& Stoermer, and Cymbella parva (Smith) Kirchner.

Representative taxa of the sediment samples with little presence in the epilithon were: Navicula opportuna Hustedt, Nitzschia garrensis Hustedt, Caloneis sp. No. 2 Posets, Sellaphora disjuncta (Hustedt) Mann, Diploneis cf. puella (Schumann) Cleve, Nitzschia pura Hustedt, Cymbella excisa Kützing, Sellaphora laevissima (Kützing) Mann, Surirella cf. roba Leclercq, Stauro- 
neis neohyalina Lange-Bertalot \& Krammer, and Pinnularia cf. brebissonii var. minuta Krammer.

\section{Commonness, dominance and richness}

The genera with the highest species richness did not correspond with those with the highest number of dominant taxa. The genus Achnanthidium was the most frequently dominant taxon both in the sediment and epilithon samples (Fig. 4), although the sediment samples showed a higher variability in the dominant genus than epilithon samples.

Four species were the most frequently dominant in the sediment samples: Achnanthidium minutissimum (Kützing) Czarnecki (13 lakes), Discostella stelligera (Cleve \& Grunow) Houk \& Klee (11 lakes), Denticula tenuis Kützing (4 lakes) and Pseudostaurosira microstriata (Marciniak) Flower (4 lakes). The most frequently dominant in the epilithon samples were: Achnanthidium minutissimum (Kützing) Czarnecki (54 lakes), Brachysira intermedia (Östrup) Lange-Bertalot (4 lakes), Encyonema minutum (Hilse) Mann (3 lakes) and Psammothidium acidoclinatum (Lange-Bertalot) Lange-Bertalot (3 lakes).
A. minutissimum is one of the most common species recorded in the epilithon of oligotrophic lakes (Linares Cuesta et al., 2007; Cantonati et al., 2012; Falasco et al., 2012). This species has usually been considered a complex due to its high morphological variability (Plates 41 to 46). Potapova and Hamilton (2007) found that despite some morphological groups of A. minutissimum apparently show a differential ecological response, it is hard to define morphological boundaries among them. Thus, the indicator potential of this morphological complex in the Pyrenean lakes, and worldwide, cannot be exploited until criteria for unambiguous morphological separation could be provided.

Rare species (frequency $<3 \%$ and maximum abundance $<3 \%$ ) showed a similar distribution in sediment and epilithon samples (Fig. 5). Thus the total of rare species was $93(22 \%)$ in sediment samples and $113(32 \%)$ in epilithon samples. Genera with the highest number of rare species approximately correspond with those with the highest species number: Eunotia, Gomphonema, Encyonema and Pinnularia in the sediment samples and Gomphonema, Naviculadicta, Nitzschia, Pinnularia and Eunotia in the epilithon samples.
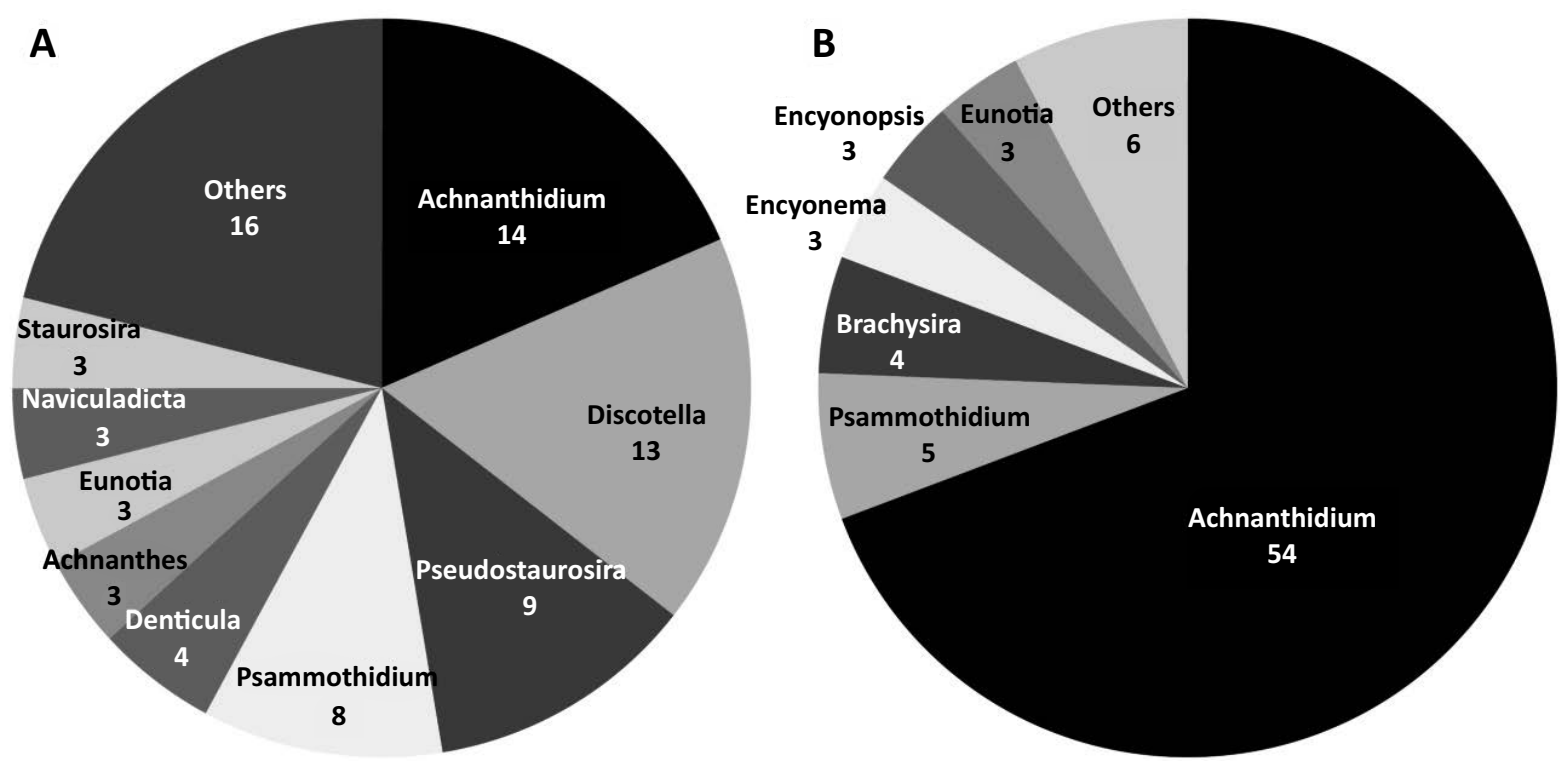

Figure 4. Frequency of lakes in which each genus was dominant in sediment (A) and epilithon samples (B) of the survey lakes. Frecuencia de lagos en los cuales cada género fue dominante en las muestras de sedimento (A) y epiliton (B) de los lagos muestreados. 

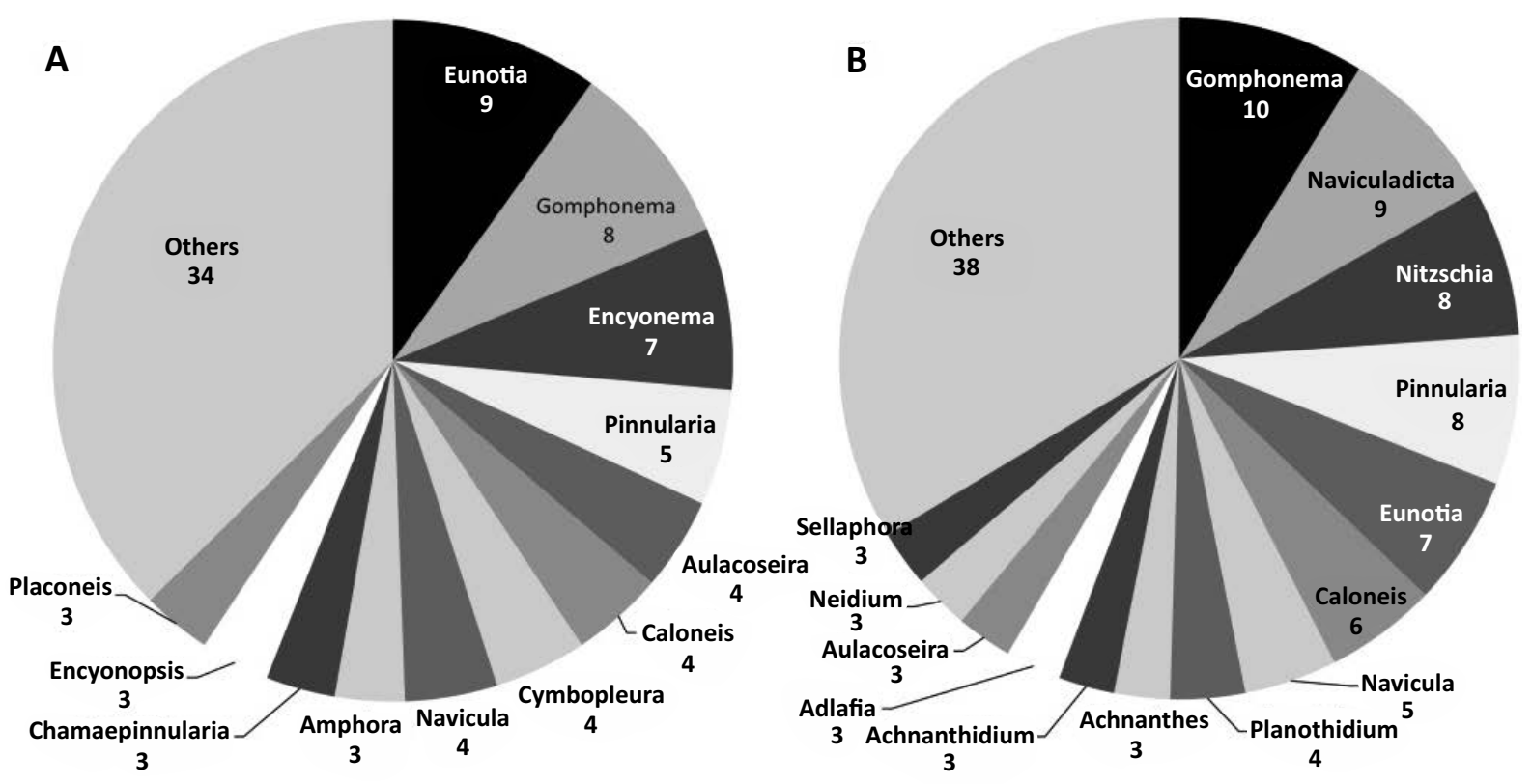

Figure 5. Rare taxa (frequency $<3 \%$ and maximum abundance $<3 \%$ ) per genus in the sediment (A) and epilithon (B) samples. Taxones raros (frecuencia <3\% y máxima abundancia <3\%) por género en las muestras de sedimento (A) y epiliton (B).

Eunotia was mainly present in acid environments (Rivera-Rondón et al., in prep.) and frequently included species with rare distribution as found elsewhere (Cantonati \& Lange-Bertalot, 2011). However, 34 Eunotia taxa were found showing a high diversity in comparison with other regions of the Iberian Peninsula (Aboal et al., 2003; Ortiz-Lerín \& Cambra, 2007). Despite the survey included a broad $\mathrm{pH}$ gradient (Table 1), the mean and many of the sampled lakes were around circumneutral conditions. Ponds were not studied, and littoral samples were collected only in the epilithic substrate. Therefore, organo-acidic environments were undersampled, which might content a good amount of additional species.

The species accumulation curves showed similar patterns of increase but richer assemblages in the sediments (Fig. 6). Richness estimators indicated that expected taxa richness could be 445-479 in sediments and 387-432 in the epilithon. As the total sediment and epilithon taxa richness found in our study were 417 and 355, respectively, we estimate that we identified about $85-93 \%$ and $79-92 \%$ of the taxa present in the sediments and epilithon, respectively. The

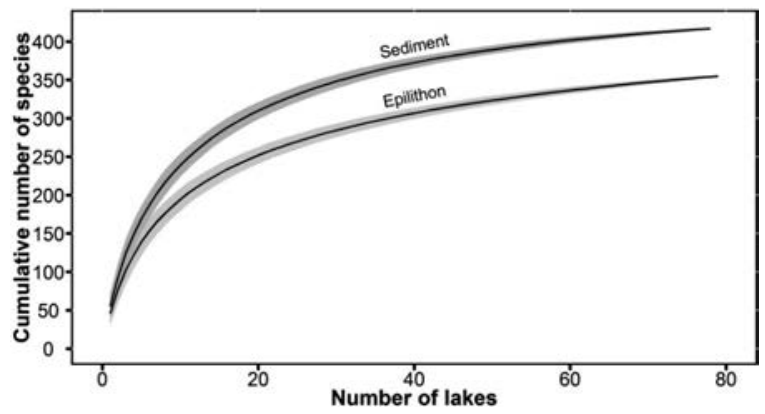

Figure 6. Comparison of the species-accumulation curves between sediment and epilithon samples. Shaded areas correspond to one standard deviation. Comparación de las curvas de acumulación de especies entre las muestras de sedimento y epiliton. Las áreas sombreadas corresponden a una desviación estándar.

diversity found is slightly higher than found in other studies carried out in sediment and epilithic samples of similar mountain lakes (Cameron et al., 1999; Clarke et al., 2005) and equivalent to other studies both in alkaline (Reavie \& Smol, 2001) and acid environments (Enache \& Prairie, 2002). However, the comparison with other studies could be strongly affected by differences in the taxonomic resolution. Despite our study in- 
clude a representative sample of Pyrenean lakes, other types of ecosystems such as small ponds, peatlands and streams, have not been extensively studied and the expected diversity of diatom in the Pyrenees is potentially higher.

\section{Taxonomical remarks}

There is still a great potential for diatom taxonomic studies in the Pyrenees. A significant amount of the taxa found $(25 \%$ of the taxa showed in the iconography) were not determined at the species level; most of them are likely new species or varieties. Among the most diverse genera, those with fewer species identified were Pinnularia (44\% of the taxa) Gomphonema (39\% of the taxa), Nitzschia (38\% of the taxa) and nonNavicula naviculoid diatoms (36\% of the taxa). Genera with little diversity but also with low species identification were Stauroneis $(60 \%$ of the taxa) and Caloneis (41\% of the taxa). Indeed, some of the species determined have not been described until recently. This is the case for several Eunotia (Cantonati \& Lange-Bertalot, 2011; Lange-Bertalot et al., 2011): Eunotia neocompacta var. vixcompacta Lange-Bertalot (Plate 33, Figs. 15-19), Eunotia catalana Lange-Bertalot \& Rivera-Rondón (Plate 24, Figs. 1-12), Eunotia novaisiae Lange-Bertalot \& Luc Ector (Plate 30, Figs. 1-10), Eunotia novaisiae var. altopyrenaica Lange-Bertalot \& Rivera-Rondón (Plate 30, Figs. 12-39), Eunotia fallacoides Lange-Bertalot \& Cantonati (Plate 35, Fig. 11). Another recently described taxa Fragilaria pararumpens LangeBertalot, Hofmann \& Werum, is common in the Pyrenean lakes and Central Europe (Hofmann et al., 2011). This species has been confused up to present with Fragilaria crotonensis Kitton and Fragilaria rumpens (Kützing) Carlson.

There were several cases of problematic identification. For example, some Eunotia specimens of a strongly acid lake showed morphological traits between E. pseudogroenlandica LangeBertalot \& Tagliaventi and E. botuliformis Wild, Nörpel \& Lange-Bertalot (Plate 31). Similarly, widespread common morphotypes were showing mixed traits, this is the case for an Encyonema showing traits of E. minutum (Hilse) Mann and
E. ventricosum (Kützing) Grunow (Plate 105, Figs. 14-31). Some small Nitzschia specimens presented the same problems (Plate 117, Figs. 7-15), even when SEM images were compared.

In conclusion, the Pyrenean lakes contain a remarkable diversity in diatoms that requires further exploration and taxonomic insights. Meanwhile, the iconography provided in this study will help in the harmonisation of current and past ecological studies.

\section{ACKNOWLEDGEMENTS}

The study was funded by EU EMERGE Programme (EVK1-CT-1999-00032) and the Spanish Government LACUS (CGL2013-45348P) and CUL-PA (Parques Nacionales 998/2013) projects. CRR was supported by a PhD scholarship of the Departamento Administrativo de Ciencia, Tecnología e Innovación de Colombia (COLCIENCIAS-ICETEX-LASPAU, 070-2007) and the Pontificia Universidad Javeriana (DJE025-2007). Professor Horst Lange-Bertalot kindly checked the species determination. We want also to thank the researchers and field assistants who assisted during the survey.

\section{REFERENCES}

ABOAL, M., A. WITKOWSKI, M.A. COBELAS, J. CAMBRA \& L. ECTOR. 2003. Floristic list of the non marine diatoms (Bacillariophyceae) of Iberian Peninsula, Balearic Islands and Canary Islands: updated taxonomy and bibliographic. In: Diatoms Monographs. Vol. 4. Witkowski, A. (ed.). A.R.G. Gantner Verlag K.G., Ruggell.

ANDERSON, N.J. 2000. Miniview: Diatoms, temperature and climatic change. European Journal of Phycology, 35: 307-314.

BIRKS, H.J.B. 1994. The importance of pollen and diatom taxonomic precision in quantitative palaeoenvironmental reconstructions. Review of Palaeobotany and Palynology, 83: 107-117.

BUKHTIYAROVA, L. \& F.E. ROUND. 1996. Revision of the genus Achnanthes sensu lato. Psammothidium, a new genus based on A. marginulatum. Diatom Research, 11: 1-30. 
CAMERON, N.G., H.J.B. BIRKS, V.J. JONES, F. BERGE, J. CATALAN, R.J. FLOWER, J. GARCIA, B. KAWECKA, K.A. KOINIG, A. MARCHETTO, P. SÁNCHEZ-CASTILLO, R. SCHMIDT, M. ŠIŠKO, N. SOLOVIEVA, E. ŠTEFKOVÁ \& M. TORO. 1999. Surface-sediment and epilithic diatom $\mathrm{pH}$ calibration sets for remote European mountain lakes (AL:PE project) and their comparison with the Surface Waters Acidification Programme (SWAP) calibration set. Journal of Paleolimnology, 22: 291-317.

CANTONATI, M., N. ANGELI, E. BERTUZZI, D. SPITALE \& H. LANGE-BERTALOT. 2012. Diatoms in springs of the Alps: spring types, environmental determinants, and substratum. Freshwater Science, 31: 499-524.

CANTONATI, M. \& H. LANGE-BERTALOT. 2011. Diatom monitors of close-to-pristine, very-low alkalinity habitats: Three new Eunotia species from springs in nature parks of the south-eastern Alps. Journal of Limnology, 70: 209-221.

CARTER, J.R. 1970. Diatoms from Andorra. Nova Hedwigia Beihefte, 31: pp. 605-633.

CATALAN, J., C.J. CURTIS \& M. KERNAN. 2009a. Remote European mountain lake ecosystems: regionalisation and ecological status. Freshwater Biology, 54: 2419-2432.

CATALAN, J., S. PLA, J. GARCIA \& L. CAMARERO. 2009b. Climate and $\mathrm{CO}_{2}$ saturation in an alpine lake throughout the Holocene. Limnology and Oceanography, 54: 2542-2552.

CATALAN, J., S. PLA-RABÉS, J. GARCÍA \& L. CAMARERO. 2014. Air temperature-driven $\mathrm{CO}_{2}$ consumption by rock weathering at short timescales: Evidence from a Holocene lake sediment record. Geochimica et Cosmochimica Acta, 136: 67-79.

CATALAN, J., M. VENTURA, M.A., C. SOLÀ \& J.M. PAGÉS. 2006. ECOES. Protocol d'avaluació de l'estat ecològic dels estanys. Agència Catalana de l'Aigua. Barcelona.

CLARKE, G., M. KERNAN, A. MARCHETTO, S. SORVARI \& J. CATALAN. 2005. Using diatoms to assess geographical patterns of change in highaltitude European lakes from pre-industrial times to the present day. Aquatic Sciences, 67: 224-236.

COLWELL, R.K. \& J.A. CODDINGTON. 1995. Estimating terrestrial biodiversity through extrapolation. In: Biodiversity: measurement and estimation. Hawksworth D.L. (ed.): 101-118. Chapman \& Hall. London.
DENICOLA, D.M., E. DE EYTO, A. WEMAERE \& K. IRVINE. 2004. Using epilithic algal communities to assess trophic status in Irish lakes. Journal of Phycology, 40: 481-495.

ENACHE, M. \& Y.T. PRAIRIE. 2002. WA-PLS diatom-based $\mathrm{pH}, \mathrm{TP}$ and DOC inference models from 42 lakes in the Abitibi clay belt area (Quebec, Canada). Journal of Paleolimnology, 27: 151-171.

FALASCO, E., L. ECTOR, E. CIACCIO, L. HOFFMANN \& F. BONA. 2012. Alpine freshwater ecosystems in a protected area: a source of diatom diversity. Hydrobiologia: 1-19.

HÅKANSSON, H. 2002. A compilation and evaluation of species in the general Stephanodiscus, $C y$ clostephanos and Cyclotella with a new genus in the family Stephanodiscaceae. Diatom Research, 17: 1-139.

HOFMANN, G., M. WERUM \& H. LANGE-BERTALOT. 2011. Diatomeen im Süßwasser-Benthos von Mitteleuropa. Bestimmungsflora Kieselalgen für die ökologische Praxis. Über 700 der häufigsten Arten und ihre Ökologie. In: Lange-Bertalot, H. (ed.). A.R.G. Gantner Verlag K.G. Ruggell.

HOUK, V. 2003. Atlas of freshwater centric diatoms with a brief key and descriptions-Part I. Melosiraceae, Orthoseiraceae, Paraliaceae and Aulacoseiraceae. Czech Phycology Supplement, 1: 1-111.

HOUK, V. \& R. KLEE. 2004. The stelligeroid taxa of the genus Cyclotella (Kützing) Brébisson (Bacillariophyceae) and their transfer into the new genus Discostella gen. nov. Diatom Research, 19: 203228.

HOUK, V., R. KLEE \& H. TANAKA. 2010. Atlas of freshwater centric diatoms with a brief key and descriptions Part III. Stephanodiscaceae A: Cyclotella, Tertiarius, Discostella. Fottea, 10 (Supplement): 1-498.

HUSTEDT, F. 1939. Diatomeen aus den Pyrenäen. Bericht der Deutschen Botanischen Gessellschaft, 56: 543-572.

JUGGINS, S. 2013. Quantitative reconstructions in palaeolimnology: New paradigm or sick science? Quaternary Science Reviews, 64: 20-32.

KRAMMER, K. 1997a. Die cymbelloiden Diatomeen-Eine Monographie der weltweit bekannten Taxa. Teil 1. Allgemeines und Encyonema Part. In: Bibliotheca Diatomologica. Vol. 36. Lange-Bertalot H. \& P. Kociolek. (eds.). J. Cramer. Berlin.

KRAMMER, K. 1997b. Die cymbelloiden Diatomeen. Ein Monographie der weltweit bekannten Taxa. Teil 2. Encyonema part., Encyonopsis and 
Cymbellopsis. In: Bibliotheca Diatomologica. Vol. 37. Lange-Bertalot H. \& P. Kociolek (eds.). J. Cramer. Berlin.

KRAMMER, K. 2000. The genus Pinnularia. In: Diatoms of Europe. Diatoms of the European Inland Waters and Comparable Habitats. Vol. 1. LangeBertalot, H. (ed.). A.R.G. Gantner Verlag K.G. Ruggell.

KRAMMER, K. 2002. Cymbella. In: Diatoms of Europe. Diatoms of the European Inland Waters and Comparable Habitats. Vol. 3. Lange-Bertalot, H. (ed.). A.R.G. Gantner Verlag K.G. Ruggell.

KRAMMER, K. 2003. Cymbopleura, Delicata, Navicymbula, Gomphocymbellopsis, Afrocymbella Supplements to cymbelloid taxa. In: Diatoms of Europe. Diatoms of the European Inland Waters and Comparable Habitats. Vol. 4. Lange-Bertalot, H. (ed.). A.R.G. Gantner Verlag K.G. Ruggell.

KRAMMER, K. \& H. LANGE-BERTALOT. 1986. Bacillariophyceae. 1. Teil: Naviculaceae. In: Süsswasser flora von Mitteleuropa. Vol. 2/1. Ettl, H., Gerloff, J., Heynig, H. \& D. Mollenhauer (eds.). Gustav Fischer Verlag. Stuttgart.

KRAMMER, K. \& H. LANGE-BERTALOT. 1988. Bacillariophyceae. 2. Teil: Bacillariaceae, Epithemiaceae, Surirellaceae. In: Süsswasserflora von Mitteleuropa. Vol. 2/2. Ettl, H., Gerloff, J., Heynig, H. $\&$ D. Mollenhauer (eds.). Gustav Fischer Verlag. Jena.

KRAMMER, K. \& H. LANGE-BERTALOT. 1991a. Bacillariophyceae. 3. Teil: Centrales, Fragilariaceae, Eunotiaceae. In: Süsswasserflora von Mitteleuropa. Vol. 2/3. Ettl, H., Gerloff, J., Heynig, H. \& D. Mollenhauer (eds.). Gustav Fischer Verlag: Stuttgart. Jena.

KRAMMER, K. \& H. LANGE-BERTALOT. 1991b. Bacillariophyceae. 4. Teil: Achnanthaceae, Kritische Ergänzungen zu Navicula (Lineolatae) und Gomphonema, Gesamtliteraturverzeichnis Teil 14. In: Süsswasserflora von Mitteleuropa. Vol. 2/4. Ettl, H., Gärtner, G., Gerloff, J., Heynig, H. \& D. Mollenhauer (eds.). Gustav Fischer Verlag: Stuttgart. Jena.

KRAMMER, K. \& H. LANGE-BERTALOT. 2004. Bacillariophyceae 4. Teil: Achnanthaceae, Kritische Erganzungen zu Navicula (Lineolatae) und Gomphonema Gesamtliteraturverzeichnis Teil 14. Second revised edition with "Ergänzungen und Revisionen" by H. Lange-Bertalot. In: Süsswasserflora von Mitteleuropa. Vol. 2/4. Ettl, H.,
Gärtner, G., Gerloff, J., Heynig, H. \& D. Mollenhauer (eds.). Spektrum Akademischer Verlag Heidelberg. Berlin.

LANGE-BERTALOT, H. 1993. 85 Neue Taxa und über 100 weitere neu definierte Taxa ergänzend zur Sübwasserflora von Mitteleuropa. In: Bibliotheca Diatomologica 27. Vol. 2/1-4. Kociolek, P. \& H. Lange-Bertalot (eds.). J. Cramer. Berlin.

LANGE-BERTALOT, H. 1997. Zur revision der Gattung Achnanthes sensu lato (Bacillariophyceae): Achnantheiopsis, eine neue Gattung mit dem Typus generis A. lanceolata, Archiv für Protistenkunde, 148: 199-208.

LANGE-BERTALOT, H. 2001. Navicula sensu stricto, 10 Genera Separated from Navicula sensu stricto, Frustulia. In: Diatoms of Europe. Diatoms of the European Inland Waters and Comparable Habitats. Vol. 4. Lange-Bertalot, H. (ed.). A.R.G. Gantner Verlag K.G. Ruggell.

LANGE-BERTALOT, H. \& K. KRAMMER. 1987. Bacillariaceae Epithemiaceae Surirellaceae. Neae und wenig bekannte Taxa, neae Kombinationen und Synonyme sowie Bemerkungen und Erganzungen zu den Naviculaceae In: Bibliotheca Diatomologica. Vol. 15. Lange-Bertalot H. \& P. Kociolek (eds.). J. Cramer. Berlin.

LANGE-BERTALOT, H. \& K. KRAMMER. 1989. Achnanthes eine Monographie der Gattung mit Definition der Gattung Cocconeis und Nachtragen $\mathrm{zu}$ den Naviculaceae. In: Bibliotheca Diatomologica. Vol. 18. Kociolek, P. \& H. Lange-Bertalot (eds.). J. Cramer. Berlin.

LANGE-BERTALOT, H. \& D. METZELTIN. 1996. Indicators of oligotrophy-800 taxa representative of three ecologically distinct lake types, Carbonate buffered-Oligodystrophic-Weakly buffered soft water. In: Iconographia Diatomologica. Annotated Diatom Micrographs. Vol 2. Lange-Bertalot H. (ed.). Koeltz Scientific Books. Königstein.

LANGE-BERTALOT, H., P. CAVACINI, N. TAGLIAVENTI \& S. ALFINITO. 2003. Diatoms of Sardinia. Rare and 76 new species in rock pools and other ephemeral waters. In: Iconographia Diatomologica. Annotated Diatom Micrographs. Vol. 12. Lange-Bertalot H. (ed.). A.R.G. Gantner Verlag K.G. Ruggell.

LANGE-BERTALOT, H., M. BAK, A. WITKOWSKI \& N. TAGLIAVENTI. 2011. Eunotia and some related genera. In: Diatoms of Europe. Diatoms of the European Inland Waters and Comparable 
Habitats. Vol. 4. Lange-Bertalot, H. (ed.). A.R.G. Gantner Verlag K.G. Ruggell.

LEVKOV, Z. 2009. Amphora sensu lato. In: Diatoms of Europe. Diatoms of the European Inland Waters and Comparable Habitats. Vol. 5. Lange-Bertalot H. (ed.). A.R.G. Gantner Verlag K.G. Ruggell. $916 \mathrm{pp}$.

LINARES CUESTA, J.E., L. OLOFSSON \& P. SÁNCHEZ CASTILLO. 2007. Epipelic diatom communities from the high mountain lakes of Sierra Nevada (Granada, Spain). Comunidades de diatomeas epipélicas en las lagunas de alta montaña de Sierra Nevada (Granada, España). Limnetica, 26(1): 99-113.

OKSANEN, J., F.A. BLANCHET, M. FRIENDLY, R. KINDT, P. LEGENDRE, D. MCGLINN, P.R. MINCHIN, R.B. O'HARA, G.L. SIMPSON, P. SOLYMOS, M.H.H. STEVENS, E. SZOECS \& H. WAGNER. 2016. Vegan: Community ecology package. R package version 2.4-0. [https://CRAN. R-project.org/package=vegan].

ORTIZ-LERIN, R. \& J. CAMBRA 2007. Distribution and taxonomic notes of Eunotia Ehrenberg 1837 (Bacillariophyceae) in rivers and streams of Northern Spain. Limnetica, 26(2): 415-434.

PÈLACHS, A., R. JULIÀ, R. PÉREZ-OBIOL, J.M. SORIANO, M.C. BAL, R. CUNILL \& J. CATALAN. 2011. Potential influence of bond events on mid-Holocene climate and vegetation in southern Pyrenees as assessed from Burg Lake LOI and pollen records. Holocene, 21: 95-104.

POTAPOVA, M. \& P.B. HAMILTON. 2007. Morphological and ecological variation within the Achnanthidium minutissimum (Bacillariophyceae) species complex. Journal of Phycology, 43: 561-575.

QUILLEN, A.K., E.E. GAISER \& E.C. GRIMM. 2013. Diatom-based paleolimnological reconstruction of regional climate and local land-use change from a protected sinkhole lake in southern Florida, USA. Journal of Paleolimnology, 49: 15-30.

R CORE TEAM (2016) R: A language and environ- ment for statistical computing. R: A language and environment for statistical computing. R Foundation for Statistical Computing. Vienna. Austria.

REAVIE, E. \& J. SMOL. 2001. Diatom-environmental relationships in 64 alkaline southeastern Ontario (Canada) lakes: a diatom-based model for water quality reconstructions. Journal of Paleolimnology, 25: 25-42.

REICHARDT, E. 1997. Taxonomische Revision des Artenkomplexes um Gomphonema pumilum (Bacillariophyceae). Nova Hedwigia, 65: 99-129.

REICHARDT, E. 1999. Zur Revision der Gattung Gomphonema. Die Arten um G. affine/insigne, $G$. angustatum/micropus, G. acuminatum sowie gomphonemoide Diatomeen aus dem Oberoligozan in Bohmen. In: Iconographia Diatomologica. Annotated Diatom Micrographs. Vol. 8. Lange-Bertalot, H. (ed.). A.R.G. Gantner Verlag K.G. Ruggell.

REICHARDT, E. 2007. Neue und wenig bekannte Gomphonema-Arten (Bacillariophyceae) mit Areolen in Doppelreihen. Nova Hedwigia, 85: 103-137.

REICHARDT, E. \& H. LANGE-BERTALOT. 1991. Taxonomische revision des artenkomplexes um Gomphonema angustatum-G. dichotomun-G. intricatum-G. vibrio und ähnliche taxa (Bacillariophyceae). Nova Hedwigia, 53: 519-544.

RIMET, F. \& A. BOUCHEZ. 2012. Biomonitoring river diatoms: Implications of taxonomic resolution. Ecological Indicators, 15: 92-99.

VAN DE VIJVER, B., L. BEYENS \& H. LANGEBERTALOT. 2004. The genus Stauroneis in the Arctic and (Sub-) Antartic Regions. In: Bibliotheca Diatomologica. Vol. 51. Lange-Bertalot H. \& P. Kociolek (eds.). J. Cramer. Berlin.

WERUM, M. \& H. LANGE-BERTALOT. 2004. Diatoms in Springs from Central Europe and elsewhere under the influence of hydrogeology and anthropogenic impacts. In: Iconographia Diatomologica. Annotated Diatom Micrographs. Vol. 13. Lange-Bertalot, H. (ed.). A.R.G. Gantner Verlag K.G. Ruggell. 
Diatom iconography of the Pyrenean lakes

\section{Index}

\begin{tabular}{|c|c|}
\hline Genera & Plates \\
\hline Achnanthes & $36,39,41,42,47,49,51,68$ \\
\hline Achnanthidium & $41-48$ \\
\hline Adlafia & 70 \\
\hline Amphipleura & 83 \\
\hline Amphora & 109 \\
\hline Asterionella & 13 \\
\hline Aulacoseira & $7-11$ \\
\hline Brachysira & $77-79$ \\
\hline Caloneis & 85 \\
\hline Cavinula & 63 \\
\hline Chamaepinnularia & 67 \\
\hline Cocconeis & 52 \\
\hline Craticula & 71 \\
\hline Cyclotella & $1,3-6$ \\
\hline Cymatopleura & 127 \\
\hline Cymbella & $93-99,102$ \\
\hline Cymbopleura & $100-102$ \\
\hline Delicata & 102 \\
\hline Denticula & 122 \\
\hline Diadesmis & 67 \\
\hline Diatoma & 12 \\
\hline Diploneis & 84 \\
\hline Discostella & 6 \\
\hline Encyonema & $104-108$ \\
\hline Encyonopsis & 102,103 \\
\hline Eolimna & 69 \\
\hline Epithemia & $123-124$ \\
\hline Eucocconeis & 36 \\
\hline Eunotia & $23-35$ \\
\hline Fallacia & 67 \\
\hline Fragilaria & $14-17$ \\
\hline Frustulia & $80-83$ \\
\hline Geissleria & 66 \\
\hline Gomphoneis & 113 \\
\hline Gomphonema & $110-116$ \\
\hline Gyrosigma & 72 \\
\hline Hannaea & 21 \\
\hline Hantzschia & 120 \\
\hline Hippodonta & 67 \\
\hline Humidophila & 67,68 \\
\hline Hygropetra & 88 \\
\hline Karayevia & 36 \\
\hline Kobayasiella & 70 \\
\hline Krasskella & 67 \\
\hline Luticola & 67 \\
\hline Meridion & 12 \\
\hline Mayamaea & 68 \\
\hline Microcostatus & 67 \\
\hline Navicula (sensu stricto) & $53-58$ \\
\hline
\end{tabular}


Diatoms of the Pyrenean lakes

\begin{tabular}{ll}
\hline \multicolumn{1}{c}{ Genera } & \multicolumn{1}{c}{ Plates } \\
\hline Navicula (sensu lato) & $65,67-70$ \\
Naviculadicta & $67-71$ \\
Neidiopsis & 73 \\
Neidium & $73-74$ \\
Nitzschia & $117-121$ \\
Nupela & 37 \\
Orthoseira & 5 \\
Peronia & 28 \\
Pinnularia & $86-92$ \\
Placoneis & 64 \\
Planothidium & 38 \\
Platessa & 38 \\
Psammothidium & $36,40-41,49-51$ \\
Pseudostaurosira & $16-19$ \\
Punctastriata & 19,20 \\
Punticulata & 2 \\
Reimeria & 108 \\
Rhopalodia & 124 \\
Rossithidium & 48 \\
Sellaphora & $59-62,69-70$ \\
Stauroforma & 16 \\
Stauroneis & $75-76$ \\
Staurosira & 16,22 \\
Staurosirella & $19-20$ \\
Stenopterobia & 126 \\
Surirella & 125,126 \\
Tabellaria & 13 \\
Tabularia & 18 \\
Ulnaria & 21 \\
\hline
\end{tabular}


Plate $1 \quad$ LM: $x 1500$

SEM: Figs. 15,18 x7000, Fig. 16 x810000, Fig. 17 x20000

Figs. 1-2 Cyclotella cf. radiosa (Grunow) Lemmermann

Figs. 3-18 Cyclotella radiosa (Grunow) Lemmermann

Fig. 1 Lake Estom, sediment PYR15

Figs. 2-12 Lake Sen, sediment PYR40

Figs. 13-14 Lake Llebreta, sediment PYR58

Figs. 15,17,18 Lake Laurenti, sediment PYR111

Fig. 16 Lake Gros de Camporrells, sediment PYR110 

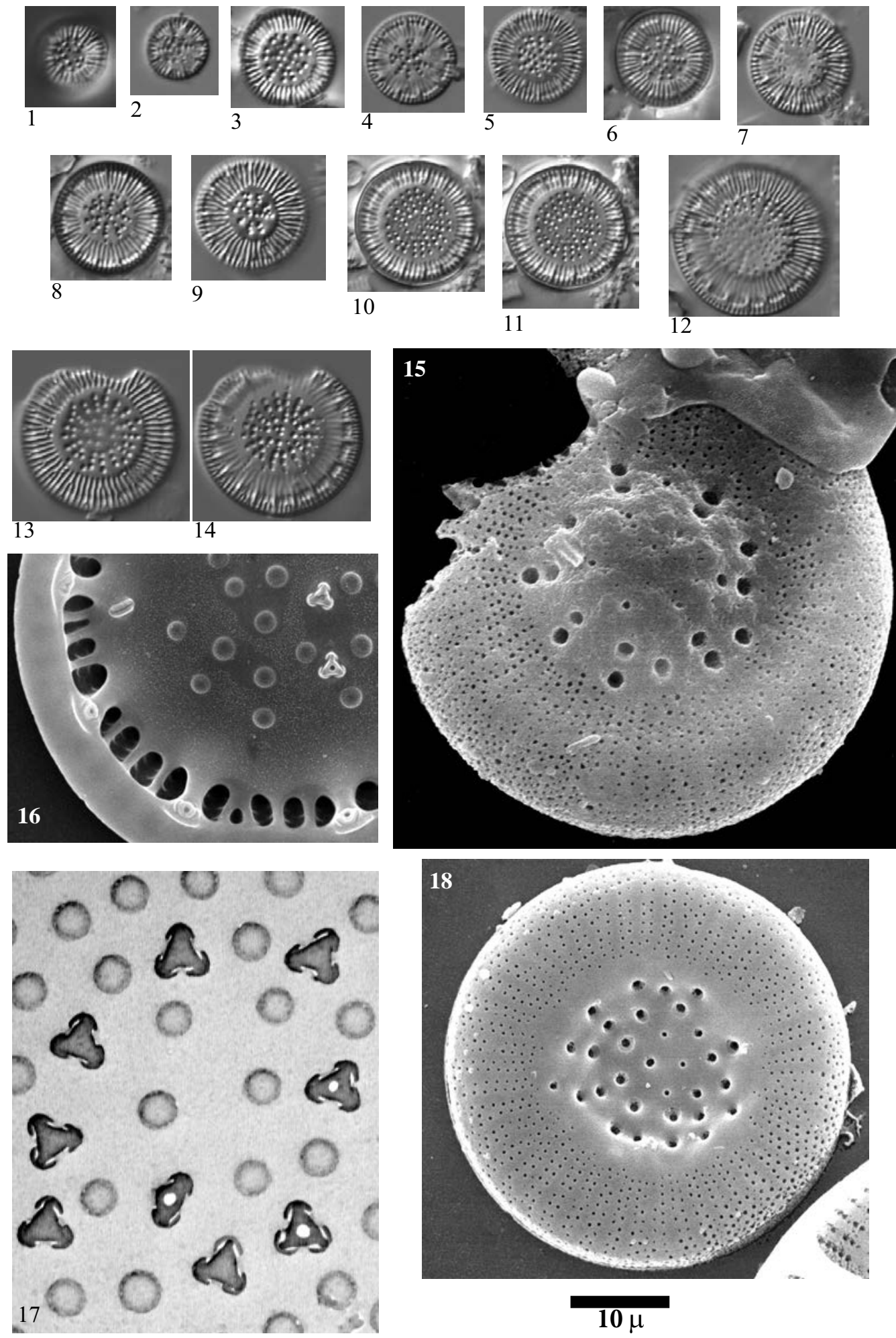
Plate 2 LM: $x 1500$

SEM: Figs. 3,5 x4000, Fig. 4 ×3000

Figs. 1-3,5 Puncticulata praetermissa (Lund) Håkansson

Figs. 4

Puncticulata cf. praetermissa (Lund) Håkansson

Figs. 1, 2 Lake Acherito, sediment PYR01

Figs. 3,5 Lake Laurenti, sediment PYR111

Fig. $4 \quad$ Lake Arnales, sediment PYR09 

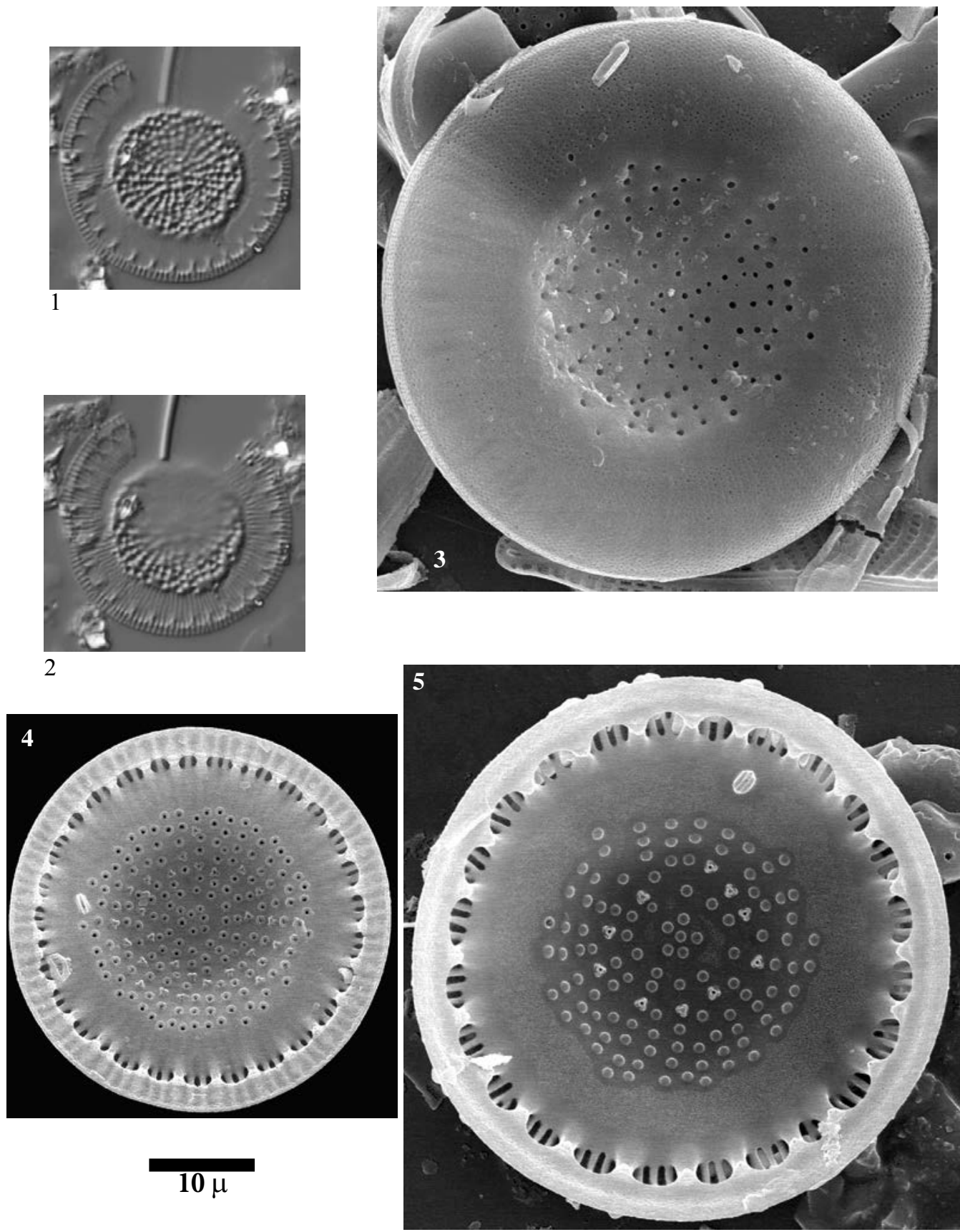
Plate 3

LM: x1500

Figs. 1-9

Cyclotella sp. No. 1 Llebreta

Figs. 1, 2, 8, 9 Lake Llebreta, sediment PYR58

Figs. 3-7

Lake Estom, sediment PYR15 


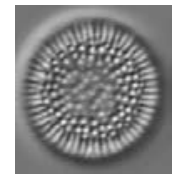

1

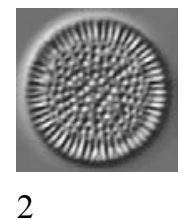

2

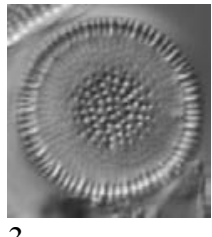

3

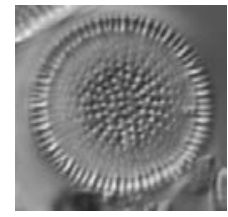

4

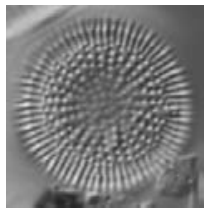

5

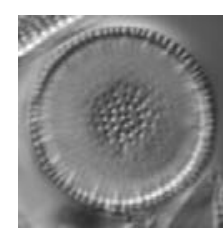

6

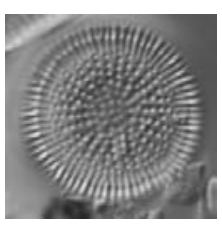

7

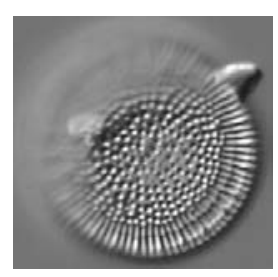

8

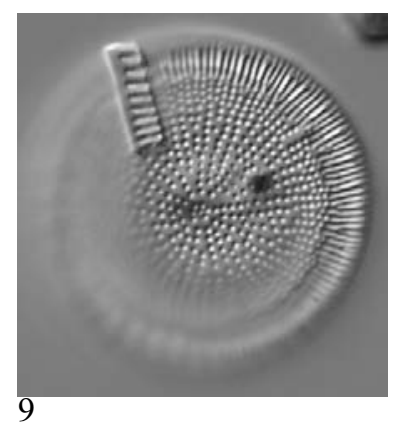


Figs. 1-12

Cyclotella intermedia (Manguin) Houk, Klee \& Tanaka

Figs. 1-7 Lake Airoto, sediment PYR73

Figs. 8-12 Lake Sen, sediment PYR40 

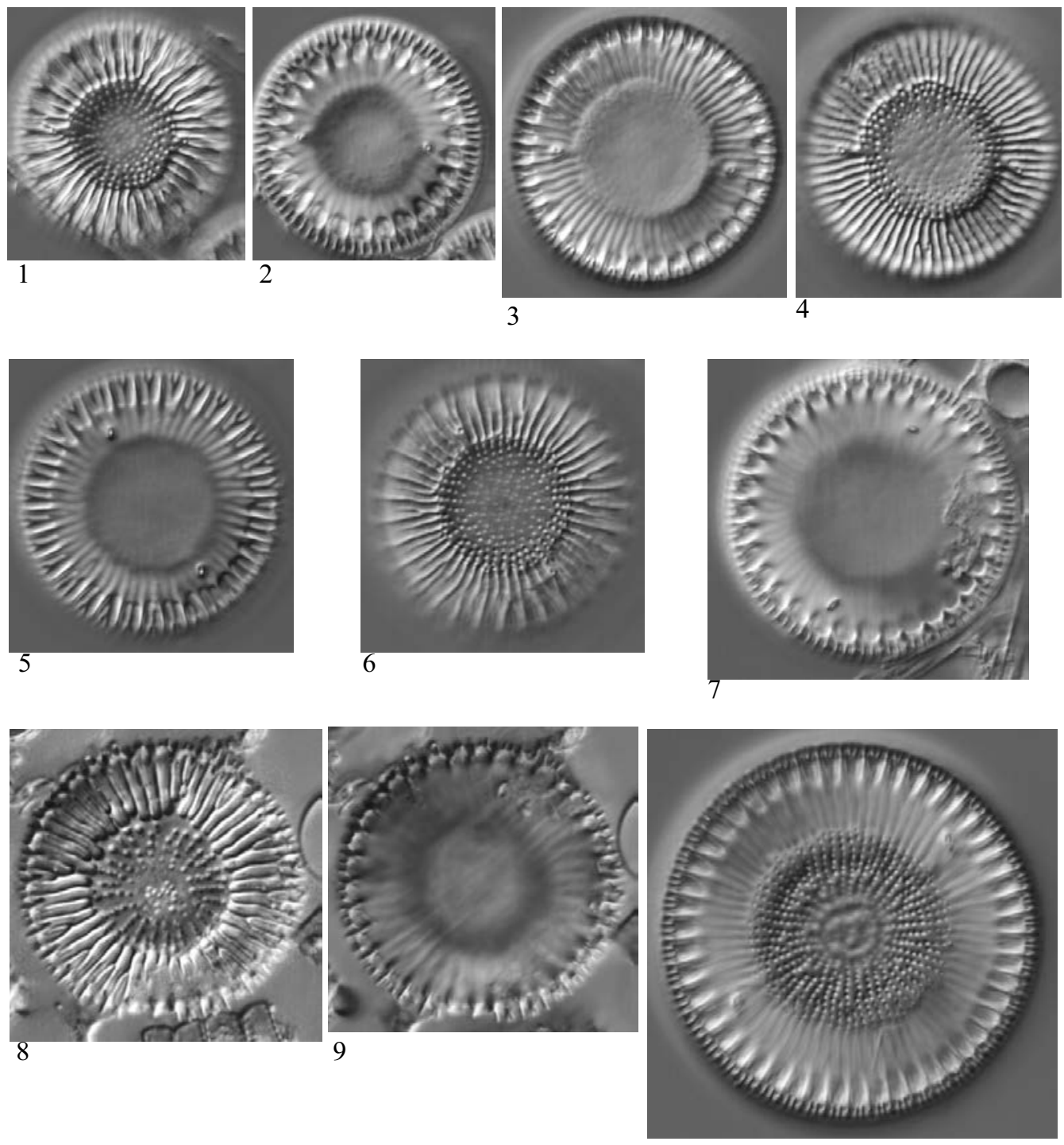

10
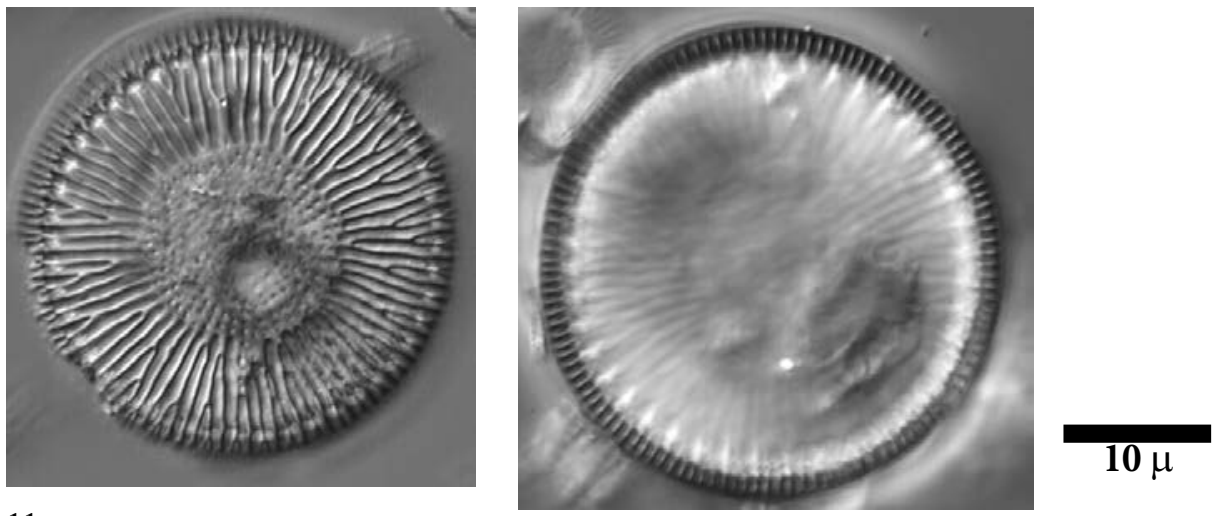
Plate 5 LM: $x 1500$

SEM: Fig. $16 \times 15000$

Figs. 1-4 Cyclotella antiqua Smith

Figs. 5-16 Cyclotella sp. No. 3 laurenti, aff. cyclopuncta Håkansson \& Carter

Fig. 17 Cyclotella ocellata Pantocsek

Fig. 18 Cyclotella cf. polymorpha Meyer \& Håkansson

Fig. 19 Cyclotella cf. comensis Grunow

Fig. 20-22 Orthosira roeseana (Rabenhorst) O’Meara

Figs. 1-2 Lake Sen, sediment PYR40

Figs. 3,4,19,20 Lake Estom, sediment PYR15

Figs. 5-11,16 Lake Laurenti, sediment PYR111

Figs. 12, 14, 15 Lake Acherito, sediment PYR27

Fig. 13

Lake Les Laquettes 1, sediment PYR27

Fig. 17 Lake Glacé, sediment PYR42

Fig. 18 Lake Puis, epilithic EpiPYR45

Figs. 21-22 Lake Monges, sediment PYR57 

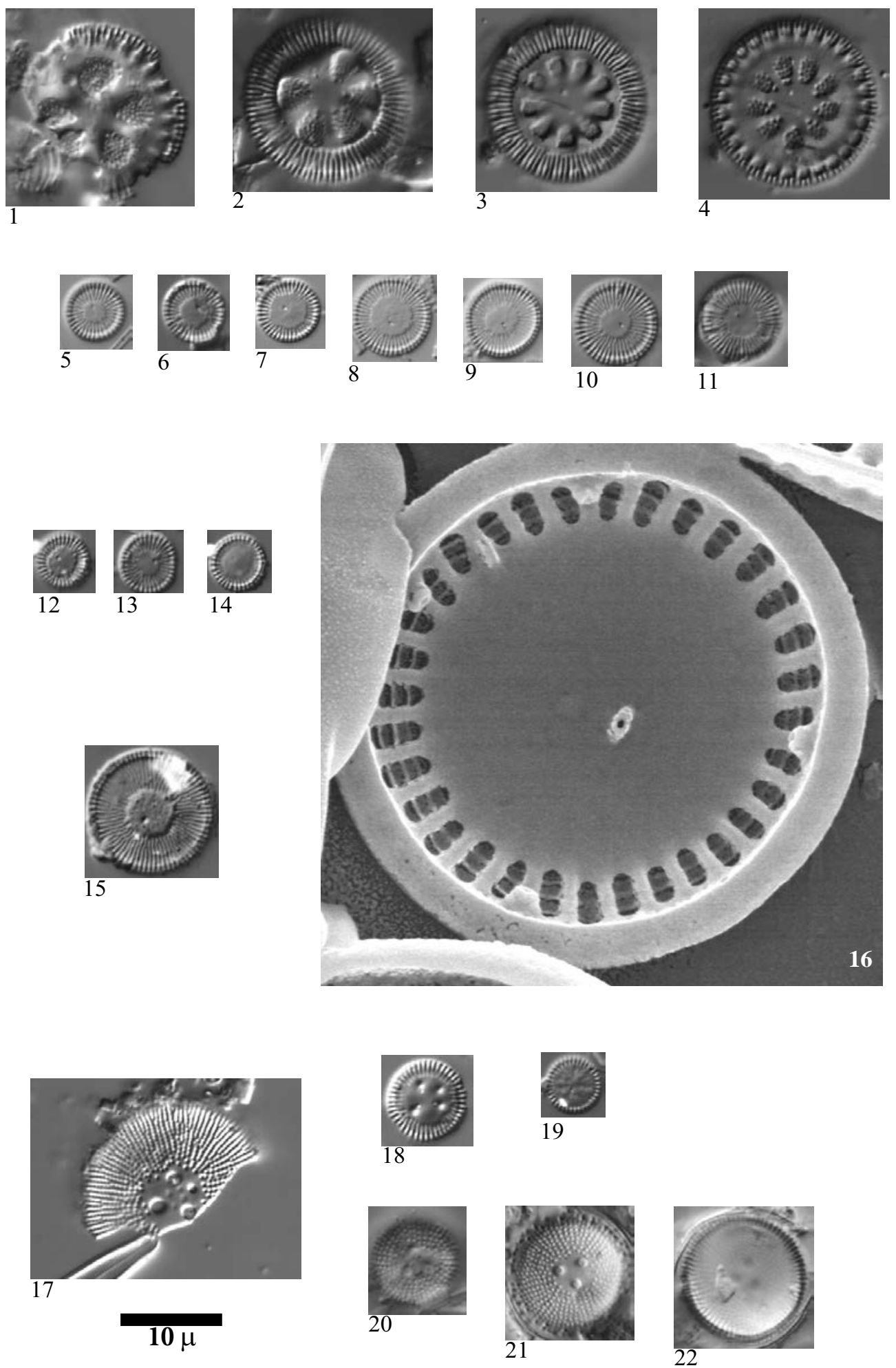
Plate 6

LM: $\mathrm{x} 1500$

SEM: $x 11000$

Fig. 1

Cyclotella sp. No. 2 Llong

Figs. 2-16

Discostella cf. pseudostelligera (Hustedt) Houk \& Klee emend.

Genkal

(Discostella sp. No. 1 Gerber)

Figs. 17-25 Discostella stelligera (Cleve \& Grunow) Houk \& Klee

Fig. $1 \quad$ Lake Llong, sediment PYR51

Fig. 3 Lake Llebreta, sediment PYR58

Figs. 4-13 Lake Gerber, sediment PYR63

Figs. 14-16 Lake Redon, sediment REDOM

Figs 17-24 Lake Port Bielh, sediment PYR28

Fig. 25 Lake Gros de Camporrells, sediment PYR110 

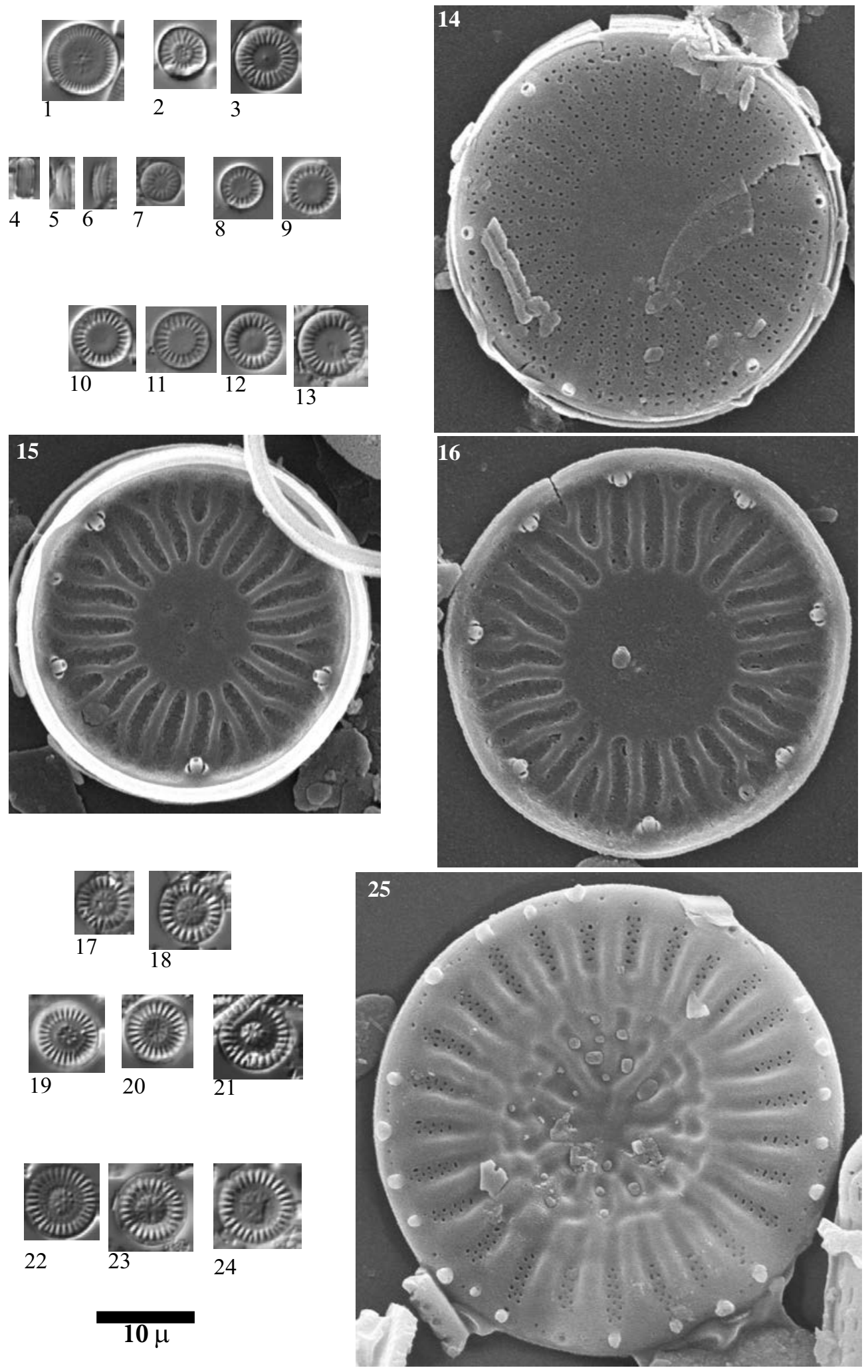

Limnetica, 36 (1): 127-395 (2017) 
Figs. 1-11 Aulacoseira pfaffiana (Reinsch) Krammer

Figs. 1-5 Lake Bleu de Rabassoles, sediment PYR112

Figs. 6, 7, 9 Lake Illa, sediment PYR66

Figs. 8, 10, 11 Lake Senó, sediment PYR84 

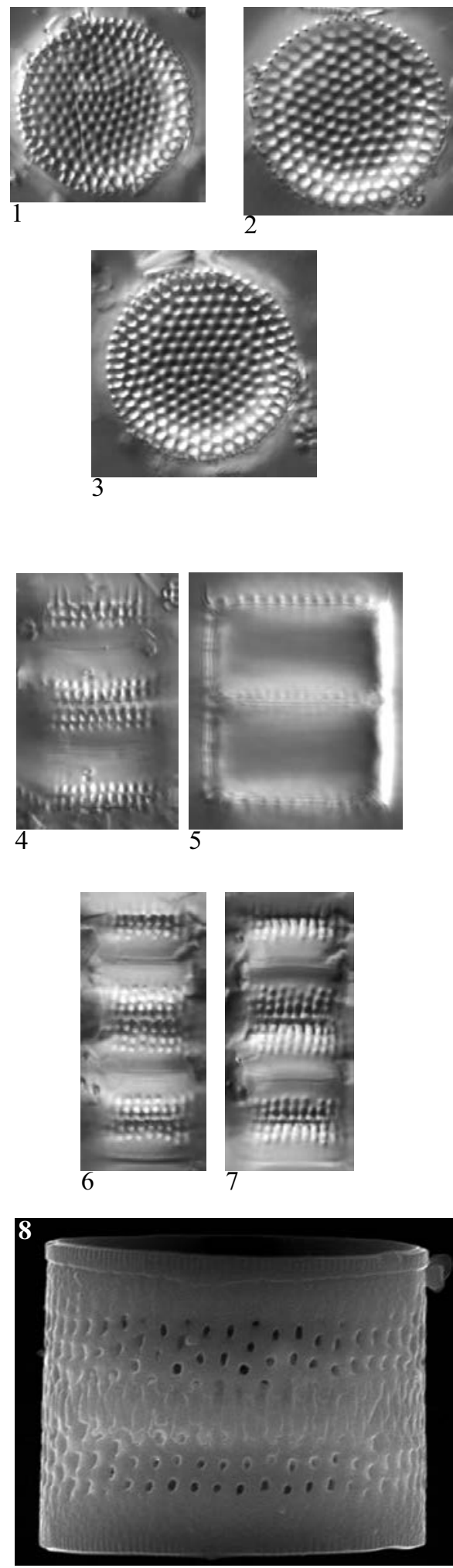
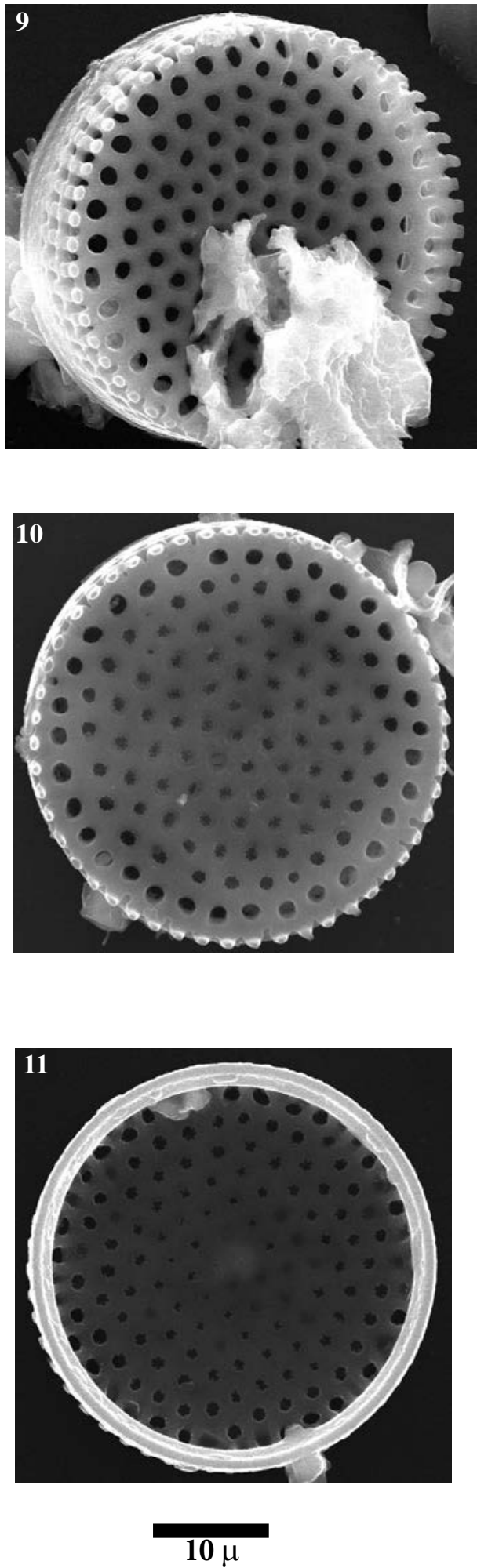
Plate 8

LM: x1500

Fig. 1

Aulacoseira granulata (Ehrenberg) Simonsen

Figs. 2-4

Aulacoseira crenulata (Ehrenberg) Thwaites

Figs. 5-6

Aulacoseira valida (Grunow) Krammer

Figs. 7-11

Aulacoseira cf. valida (Grunow) Krammer

Figs. 12-15

Aulacoseira cf. subarctica (O. Müller) Haworth

Figs. 16-20

Aulacoseira perglabra (Østrup) Haworth

Fig. 1

Lake Forcat Inferior, sediment PYR77

Figs. 2-6, 18- Lake Albe, sediment PYR96

22

Figs. 7-8 Lake Siscar, sediment PYR126

Figs. 9-15 Lake Ensangents Superior, sediment PYR106

Figs. 16-17 Lake Llong, sediment PYR59 

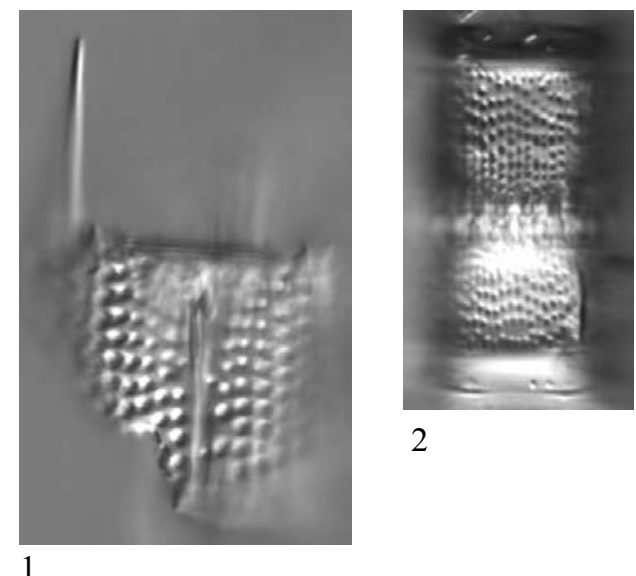

2
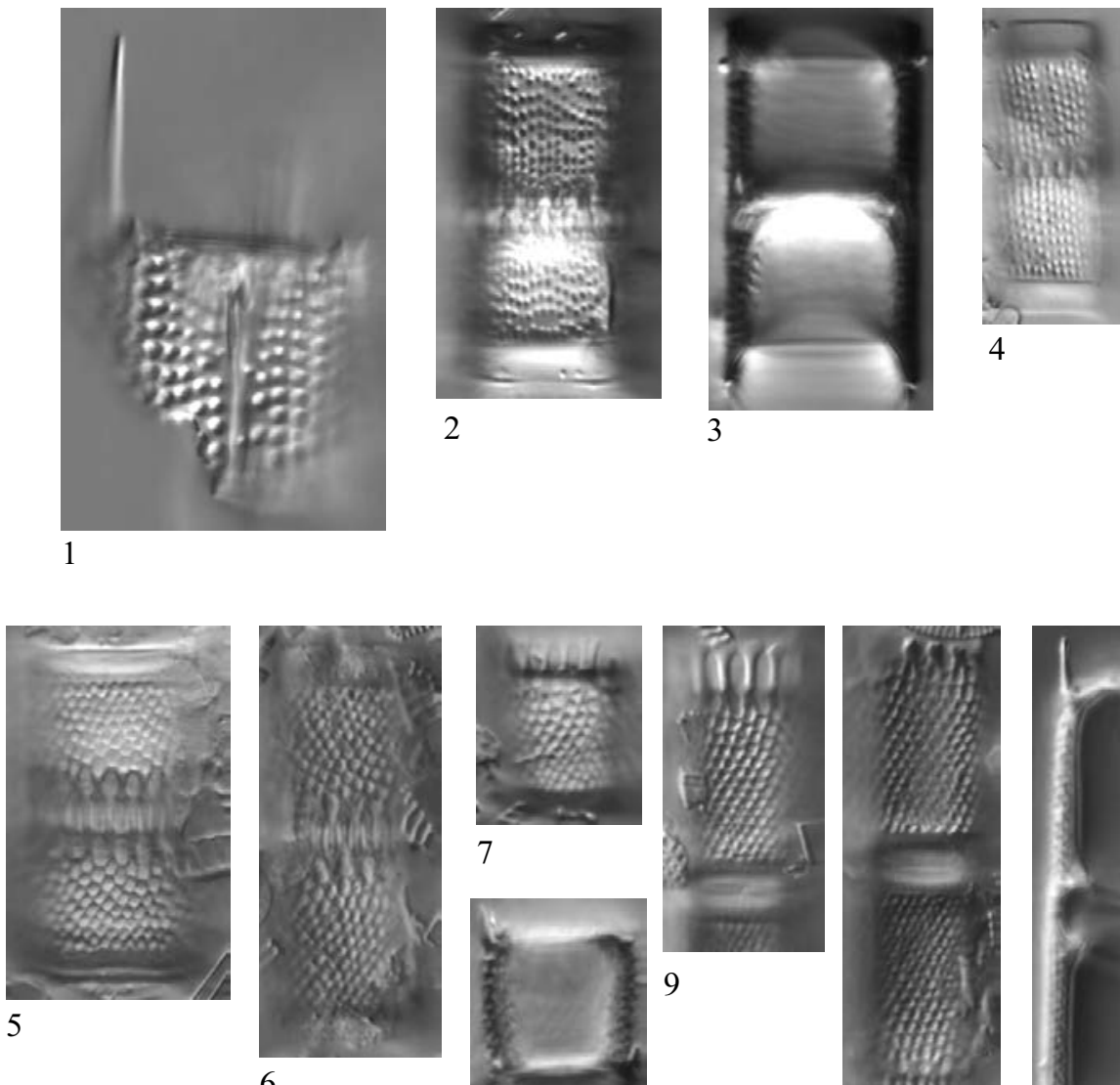

7
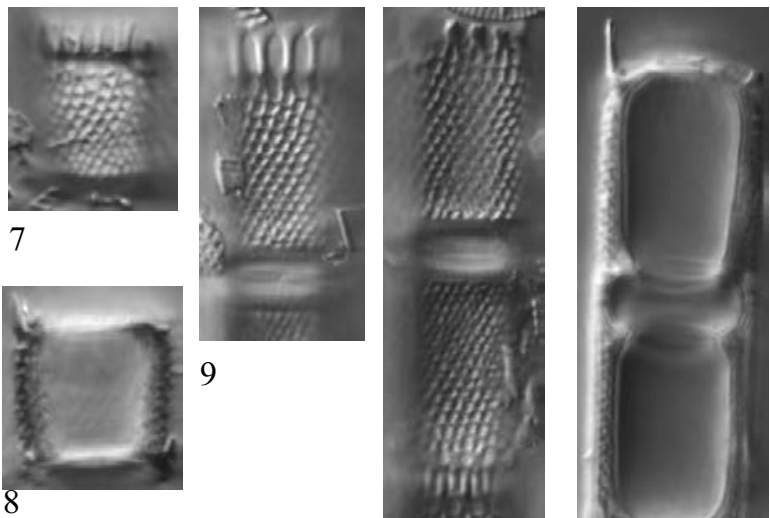

5

6

$$
8
$$

9
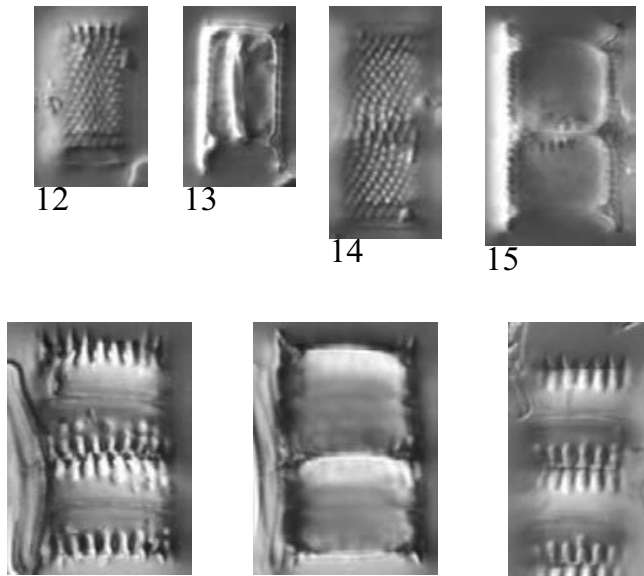

16

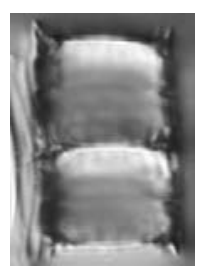

17

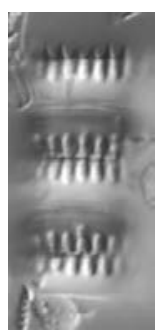

18
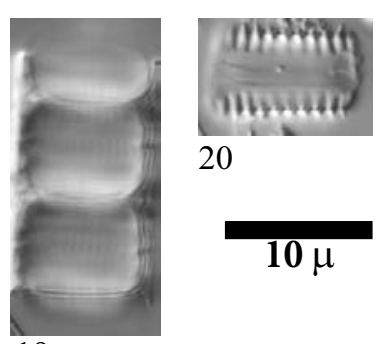
Plate $9 \quad$ LM: $x 1500$

SEM: Figs. 8-10 x4000, Fig. 11 x10000

Figs. 1-11 Aulacoseira lirata (Ehrenberg) Ross

Figs. 1-2, 8-11 Lake Redon, sediment REDOM

Figs. 3-4, 7 Lake Albe, sediment PYR96

Figs. 5-6 Lake Posets, sediment PYR42 

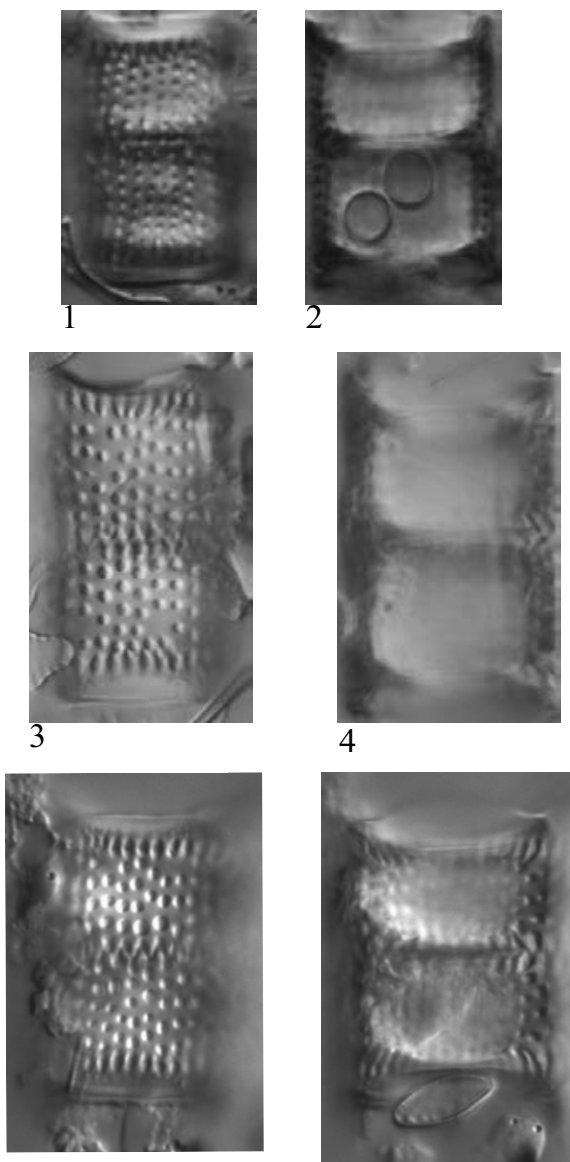

5
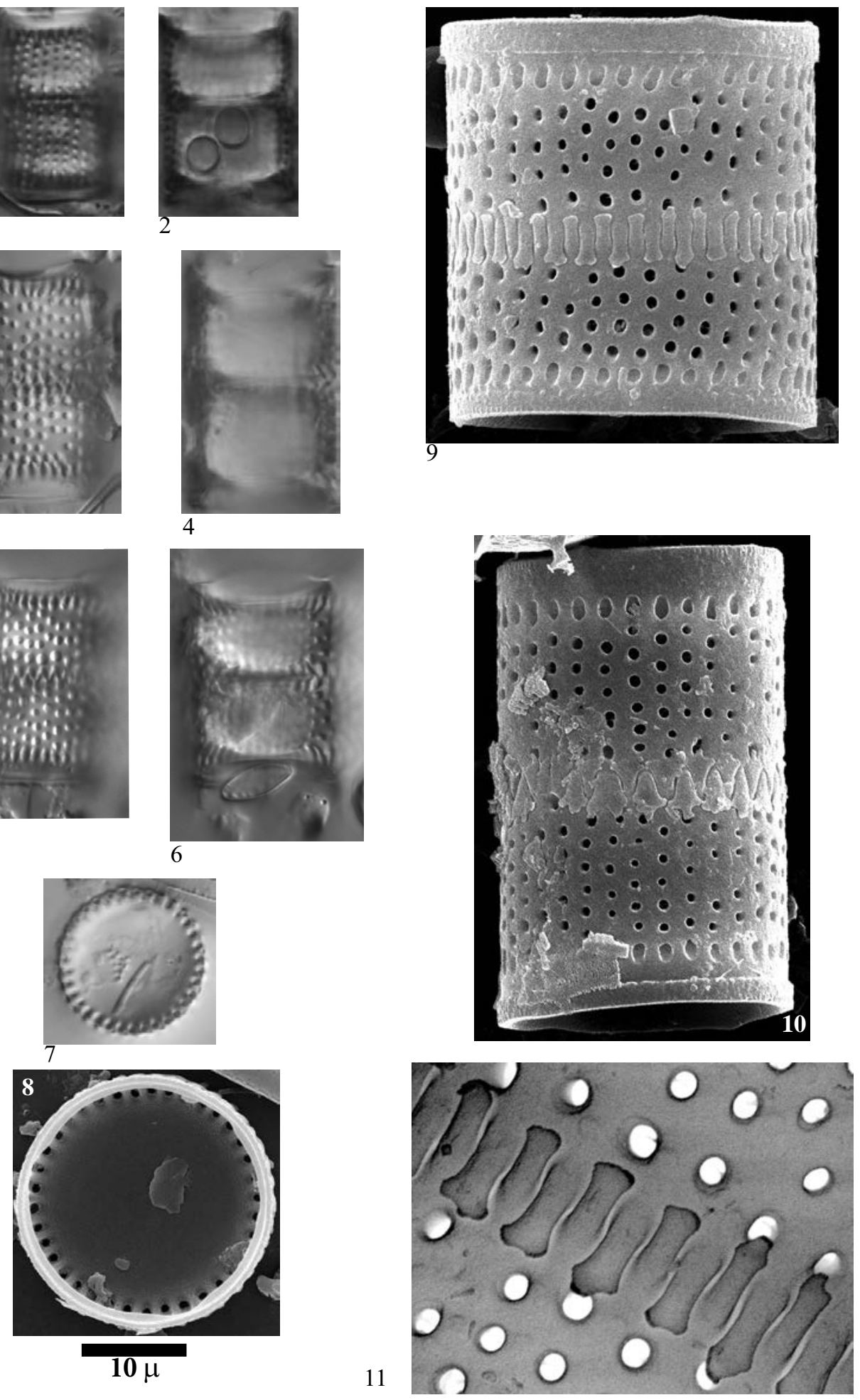
Figs. 1-2

Figs. 3-6

Figs. 7-22

Figs. 23-44

Figs. 1-4

Figs. 5-6

Figs. 7-22

Figs. 23-24

Figs. 25-28

Figs. 29-32

Figs. 33-40

Figs. 41-44
Aulacoseira cf. lirata var. biseriata (Grunow) Haworth

Aulacoseira cf. ambigua (Grunow) Simonsen

Aulacoseira sp. No. 1 Gerber

Aulacoseira cf. alpigena (Grunow) Krammer

Lake Redón, sediment REDOM

Lake Cregueña, sediment PYR49

Lake Gerber, sediment PYR63

Lake Les Laquettes 1, sediment PYR27

Lake Forcat Inferior, sediment PYR77

Lake Pica, sediment PYR100

Lake Bleu de Rabassoles, sediment PYR112

Lake Negre, sediment PYR79 

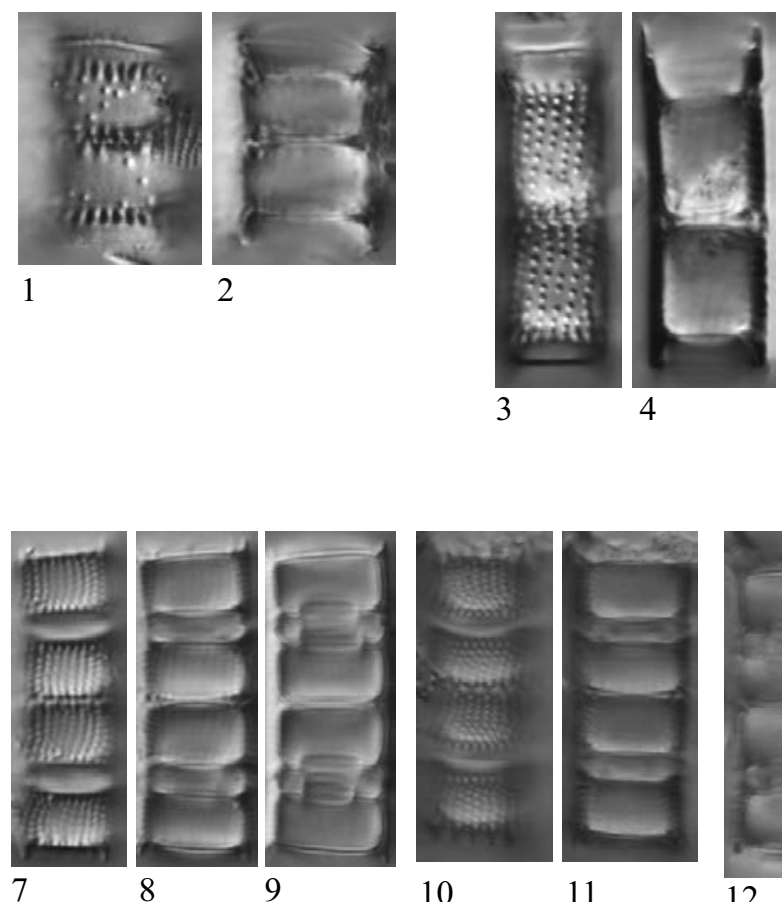

11
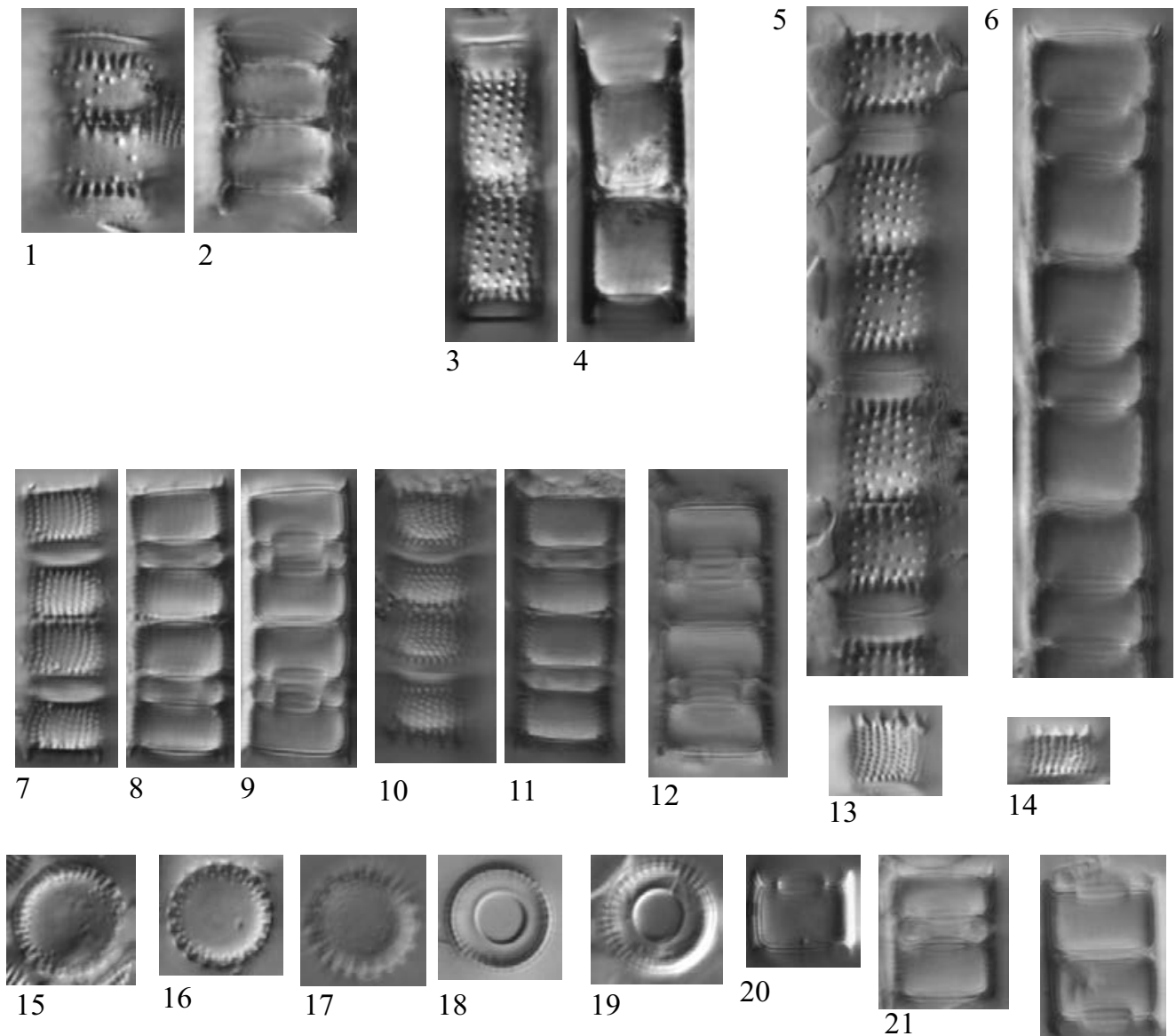

13

14
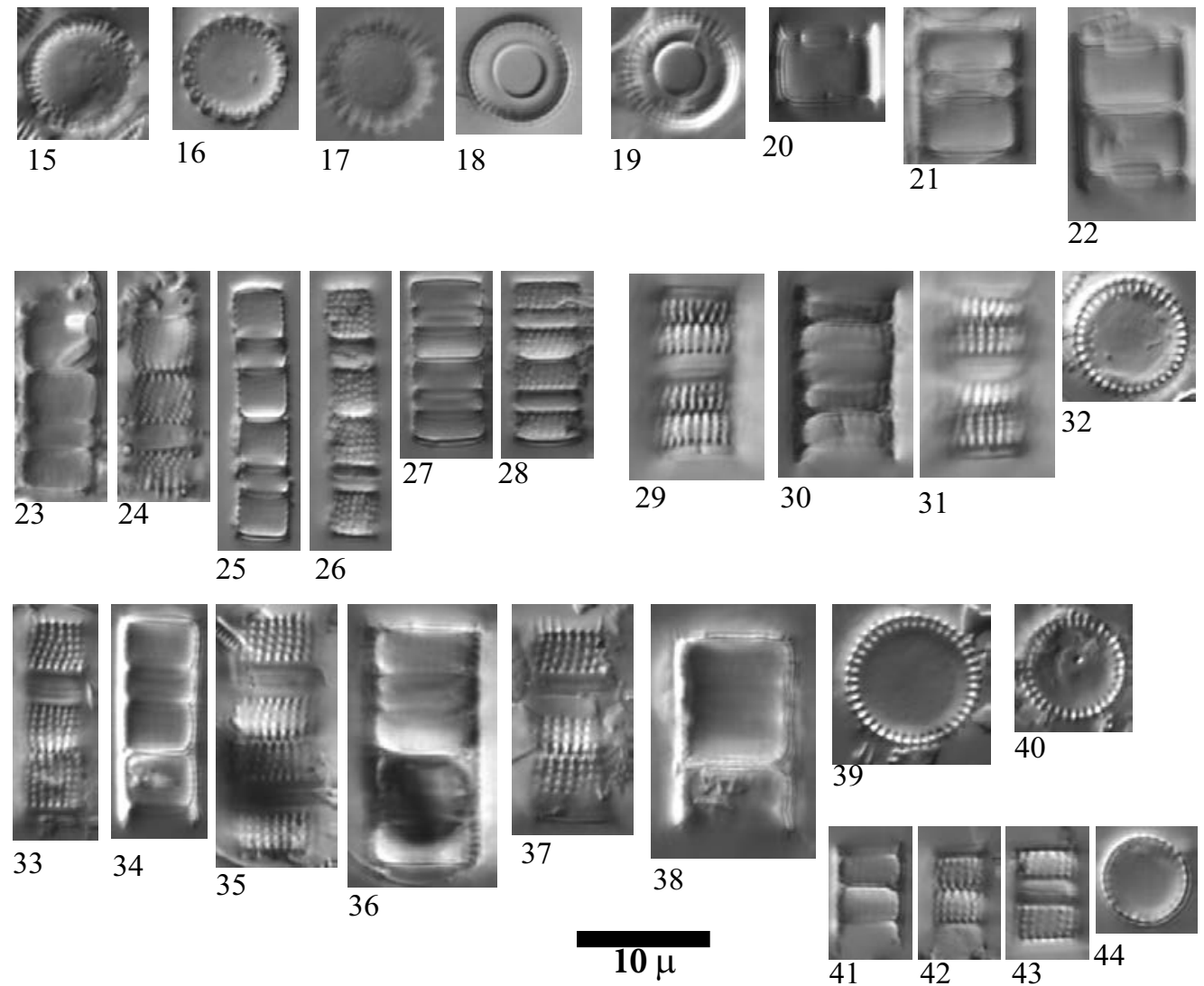

Limnetica, 36 (1): 127-395 (2017) 
Plate $11 \quad$ LM: $x 1500$

SEM: $\mathrm{x} 4000$

Figs. 1-5 Aulacoseira distans (Ehrenberg) Simonsen

Fig. 6

Aulacoseira cf. distans (Ehrenberg) Simonsen

Figs. 7-9

Aulacoseira humilis (Cleve-Euler) Genkal \& Trifonova in Trifonova \& Genkal

Figs. 10-16 Aulacoseira sp.

Figs. 17-19 Aulacoseira cf. nygaardii (Camburn) Camburn \& Charles

Figs. 1-5 Lake Negre, sediment PYR79

Fig. 6 Lake Redon, sediment REDOM

Figs. 7-10, Lake Albe, sediment PYR96

14-16

Figs. 11-13 Lake Llong, sediment PYR59

Figs. 17-19 Lake Sotllo, sediment PYR89 

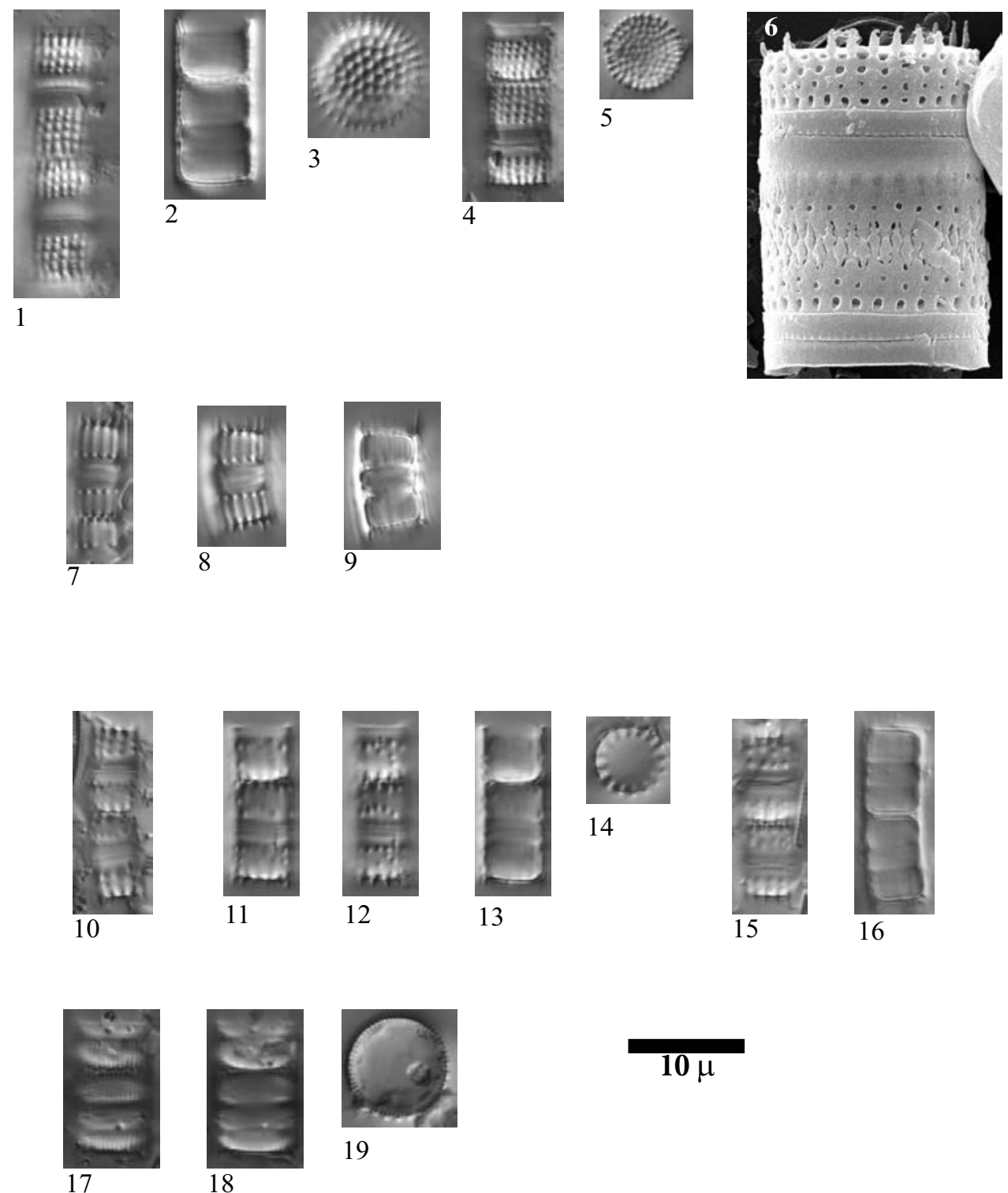
Fig. 1

Figs. 2-8

Figs. 9-14

Fig. 15

Figs. 16-17

Figs. 18-22
Diatoma vulgaris Bory

Diatoma mesodon Kützing

Diatoma hyemalis (Roth) Heiberg sensu Krammer \& Lange-Bertalot 1991

Diatoma sp. No. 1 Estom

Meridion circulare var. constrictum (Ralfs) Van Heurck sensu

Krammer \& Lange-Bertalot 1991

Meridion circulare (Greville) Agardh
Fig. 1

Figs. 2, 7, 18

Fig. 3

Figs. $4,5,8,12$

Figs. 6, 19, 22

Figs. 9-11, 13-15

Fig. 16

Fig. 17

Fig. 20

Fig. 21
Lake Roumassot, sediment PYR04

Lake Arratille, sediment PYR11

Lake Posets, sediment PYR42

Lake Llebreta, sediment PYR58

Lake Laurenti, sediment PYR111

Lake Estom, sediment PYR15

Lake Labas, sediment PYR63

Lake Estelat, sediment PYR120

Lake Baiau Superior, sediment PYR76

Lake Burg, sediment BURG1210 

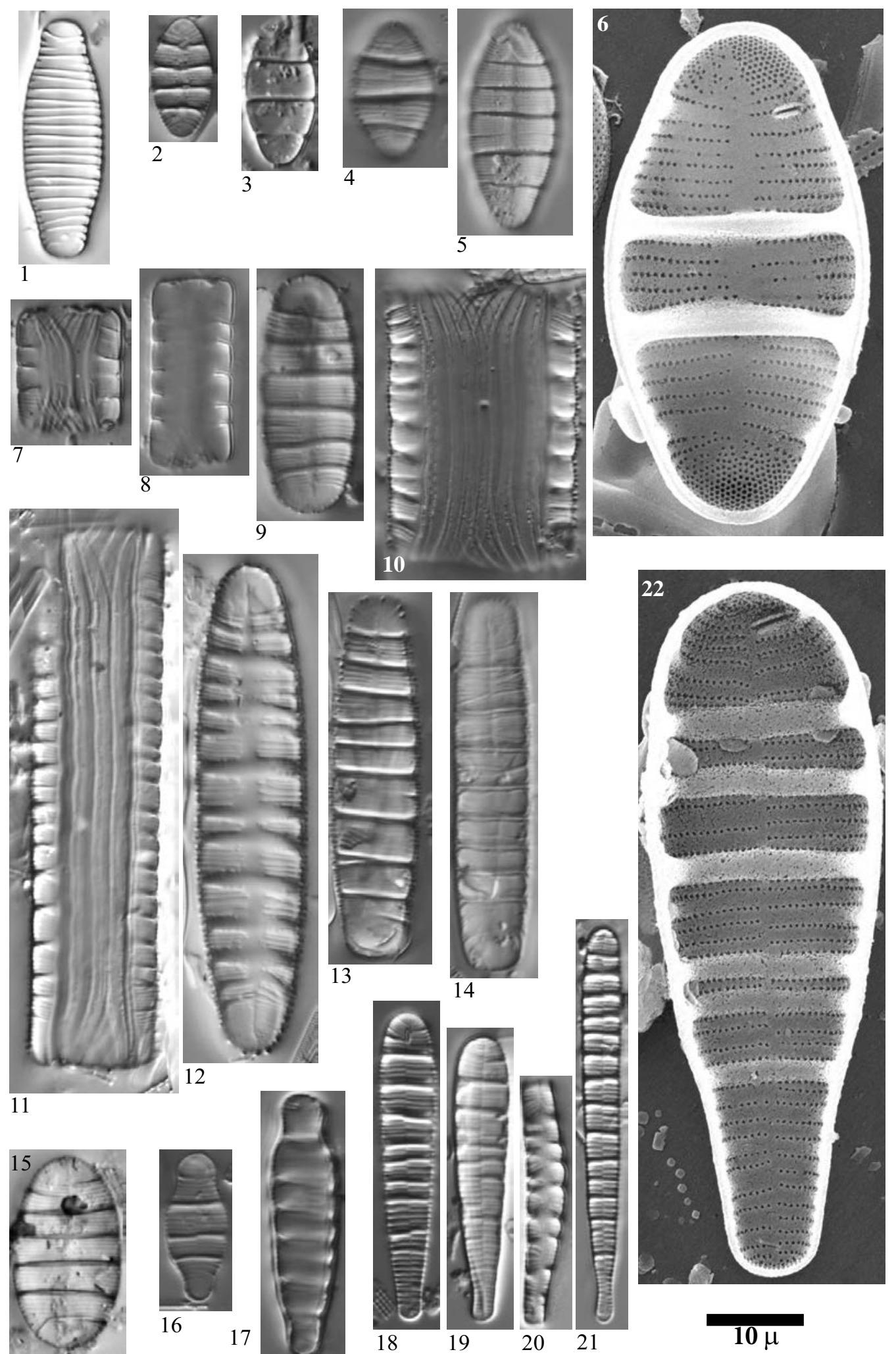

Limnetica, 36 (1): 127-395 (2017) 
Plate $13 \quad$ LM: $x 1500$

Figs. 1-23 Tabellaria flocculosa (Roth) Kützing

Figs. 23-25 Tabellaria ventricosa Kützing

Fig. $27 \quad$ Tabellaria fenestrata (Lyngbye) Kützing

Figs. 28-29 Asterionella formosa Hassall

Figs. 1-4 Lake Pica, epilithic EpiPYR100

Figs. 5-8, 11- Lake Senó, epilithic EpiPYR84

16

Figs. 9-10 Lake Bleu de Rabassoles, epilithic EpiPYR112

Figs. 17, 19, 21 Lake L'Estagnol, epilithic EpiPYR119

Fig. 18 Lake L'Estagnol, sediment PYR119

Figs. 22, $27 \quad$ Lake Llebreta, sediment PYR58

Figs. 23, 24, 26 Lake Senó, sediment PYR84

Fig. $25 \quad$ Lake Senó, epilithic EpiPYR84

Fig. 28 Lake Bersau, sediment PYR03

Fig. 29 Lake Airoto, sediment PYR73 

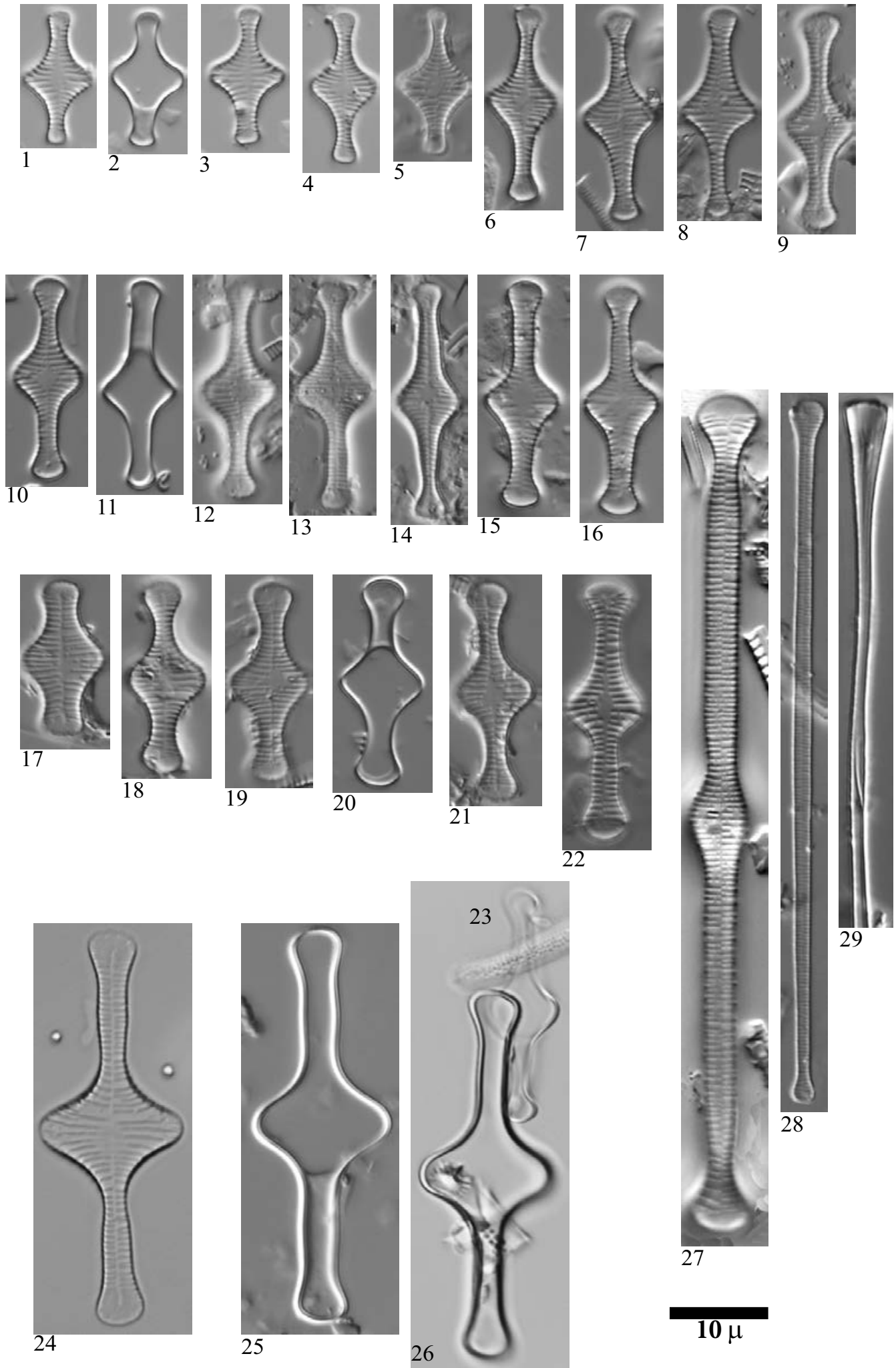

29

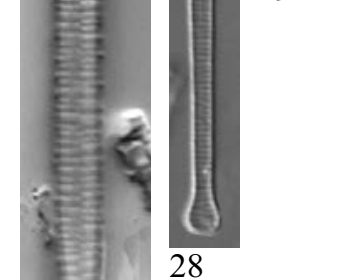


Plate $14 \quad$ LM: $x 1500$

SEM: Figs. $4-5$ x2000, Fig. 22 x6000, Fig. 25 x9000, Figs. 23-24, $26-27 \times 10000$

Fig. 1-2

Fragila sp. No. 1 Airoto

Figs. 3-5,

Fragilaria delicatissima (Smith) Lange-Bertalot

23-28

Figs. 6-13 Fragilaria sp. (?nanoides)

Figs. 14-17 Fragilaria cf. gracilis Østrup

Figs. 18-19 Fragilaria cf. tenera (Smith) Lange-Bertalot

Fig. 20

Fragilaria saxoplanctonica nom. prov.

Fig. 21

Fragilaria cf. nanana Lange-Bertalot

Fig. 22

Fragilaria cf. nanoides Lange-Bertalot

Figs. 1, 10-13 Lake Airoto, sediment PYR73

Fig. 2

Lake Les Laquettes, sediment PYR27

Figs. 3-5, 23- Lake Redón, sediment REDOM

28

Figs. 6-9 Lake Sen, sediment PYR40

Figs. 14, 18, 20 Lake Bersau, sediment PYR03

Fig. 19 Lake Posets, sediment PYR42

Fig. $21 \quad$ Lake Tourrat, sediment PYR23

Fig. 22 Lake Monges, sediment PYR57 


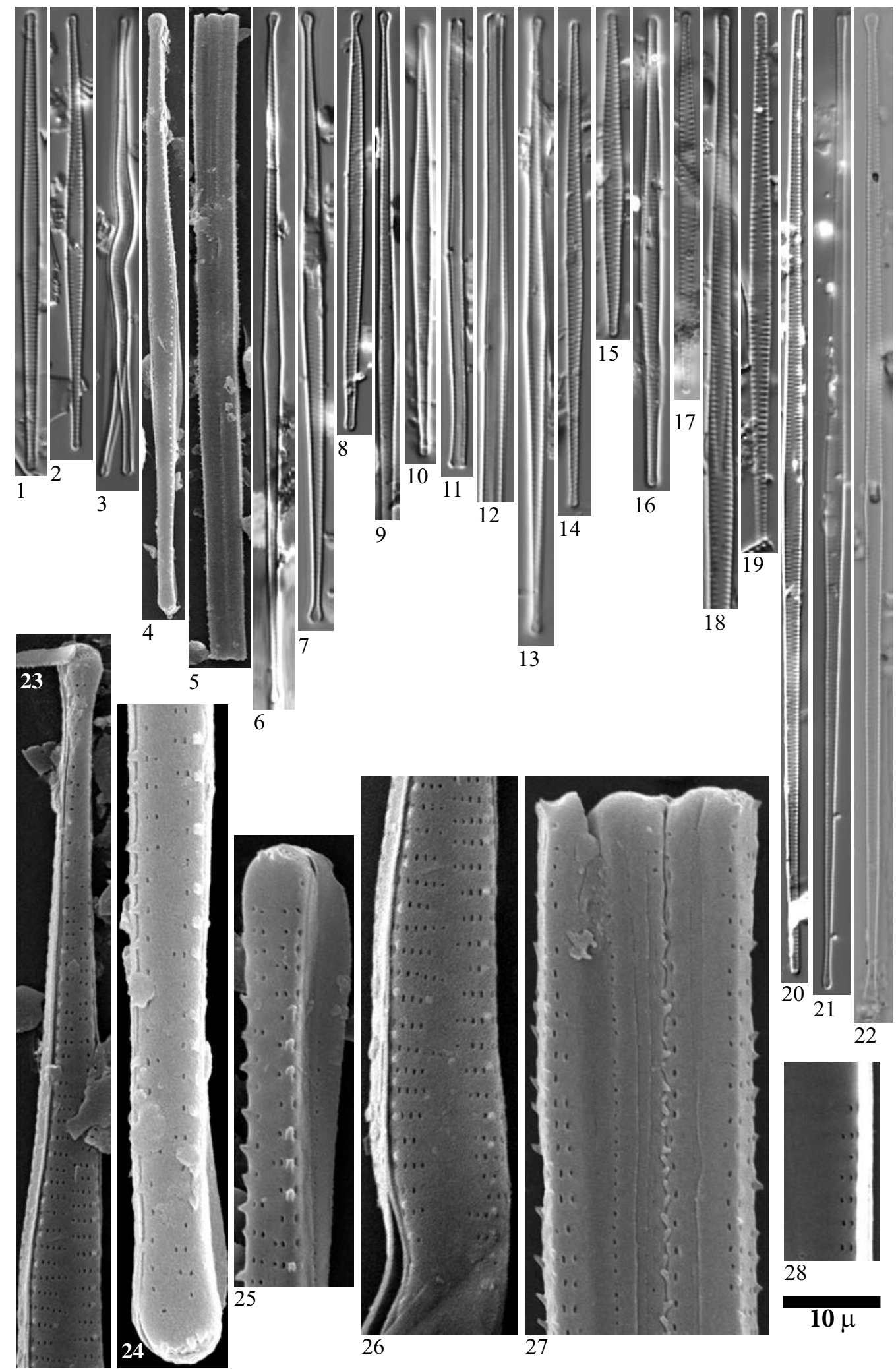


Figs. 1-27 Fragilaria cf. pararumpens Lange-Bertalot, Hofmann \& Werum

Figs. 28-31 Fragilaria spp.

Fig. $32 \quad$ Fragilaria sp.

Fig. 33-41 Fragilaria sp. No. 2 Bersau

Fig. $42 \quad$ Fragilaria sp. No. 3 Airoto

Fig. $43 \quad$ Fragilaria cf. rumpens (Kützing) Carlson

Figs. 44-46 Fragilaria cf. perminuta (Grunow) Lange-Bertalot

Figs. 47-48 Fragilaria cf. vaucheriae (Kützing) Petersen

Fig. $49 \quad$ Fragilaria cf. recapitellata Lange-Bertalot \& Metzeltin

Fig. $50 \quad$ Fragilaria sp. No. 4 Laquettes

Figs. 1-15, Lake Bersau, sediment PYR03 $18-28$, $32-41$

Figs. 16-17 Lake Burg, sediment BURG1007

Figs. 29-31 Lake Sen, sediment PYR40

Figs. 42 Lake Airoto, sediment PYR73

Fig. 43 Lake Eriste, sediment PYR43

Figs. 44-45 Lake Inferior de la Gallina, sediment PYR87

Fig. 46 Lake Bleu de Rabassoles, sediment PYR112

Fig. 47-49 Lake Llebreta, sediment PYR58

Fig. $50 \quad$ Lake Les Laquettes, sediment PYR27 

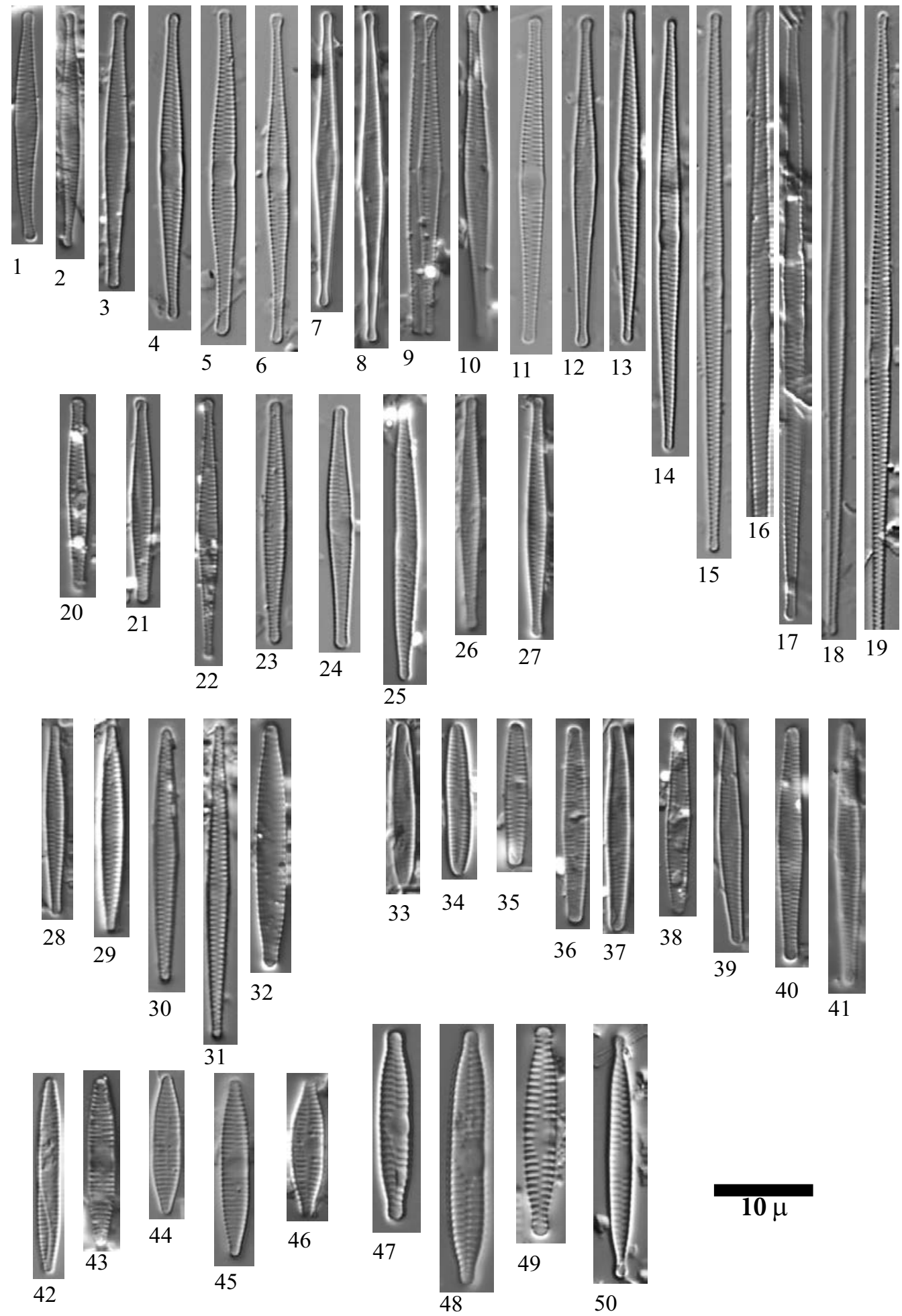
Plate $16 \quad$ LM: x1500

SEM: Fig. 11 x3500, Fig. 12 x3000

Fig. 1 Fragilaria sp. No. 5 Aube, aff. F. nevadensis Linares-Cuesta \& Sanchez-Castillo

Figs. 2-8 Fragilaria alpestris Krasske

Figs. 9-11 Fragilaria sp. No. 6 Blaou

Figs. 12-14 Stauroforma cf. exiguiformis (Lange-Bertalot) Flower, Jones \& Round

Fig. $15 \quad$ Fragilaria cf. mesolepta Rabenhorst

Figs. 16-17 Fragilaria sp. No. 7 Arratille

Figs. 18-19 Staurosira parasitoides Lange-Bertalot, Schmidt \& Klee

Fig. $20 \quad$ Pseudostaurosira cf. microstriata (Marciniak) Flower

Figs 21-22 Pseudostaurosira parasitica (Smith) Morales

Fig. 23 Pseudostaurosira parasitica var. subconstricta (Grunow) Morales

Fig. $1 \quad$ Lake Senó, sediment PYR84

Figs. 2-8 Lake Helado del Monte Perdido, sediment PYR19

Figs. 9-10 Lake Blaou, epilithic EpiPYR94

Fig. $11 \quad$ Lake Port Bielh, sediment PYR28

Fig. 12 Lake Redon, sediment REDOM

Fig. 13 Lake Plan, sediment PYR69

Fig. 14 Lake Romedo de Dalt, sediment PYR85

Fig. 15 Lake Burg, sediment BURG1169

Figs. 16-17, 20 Lake Arratille, sediment PYR11

Figs. 18-19, 21 Lake Acherito, sediment PYR01

Fig. 22 Lake Sen, sediment PYR40

Fig. 23 Lake Posets, sediment PYR42 

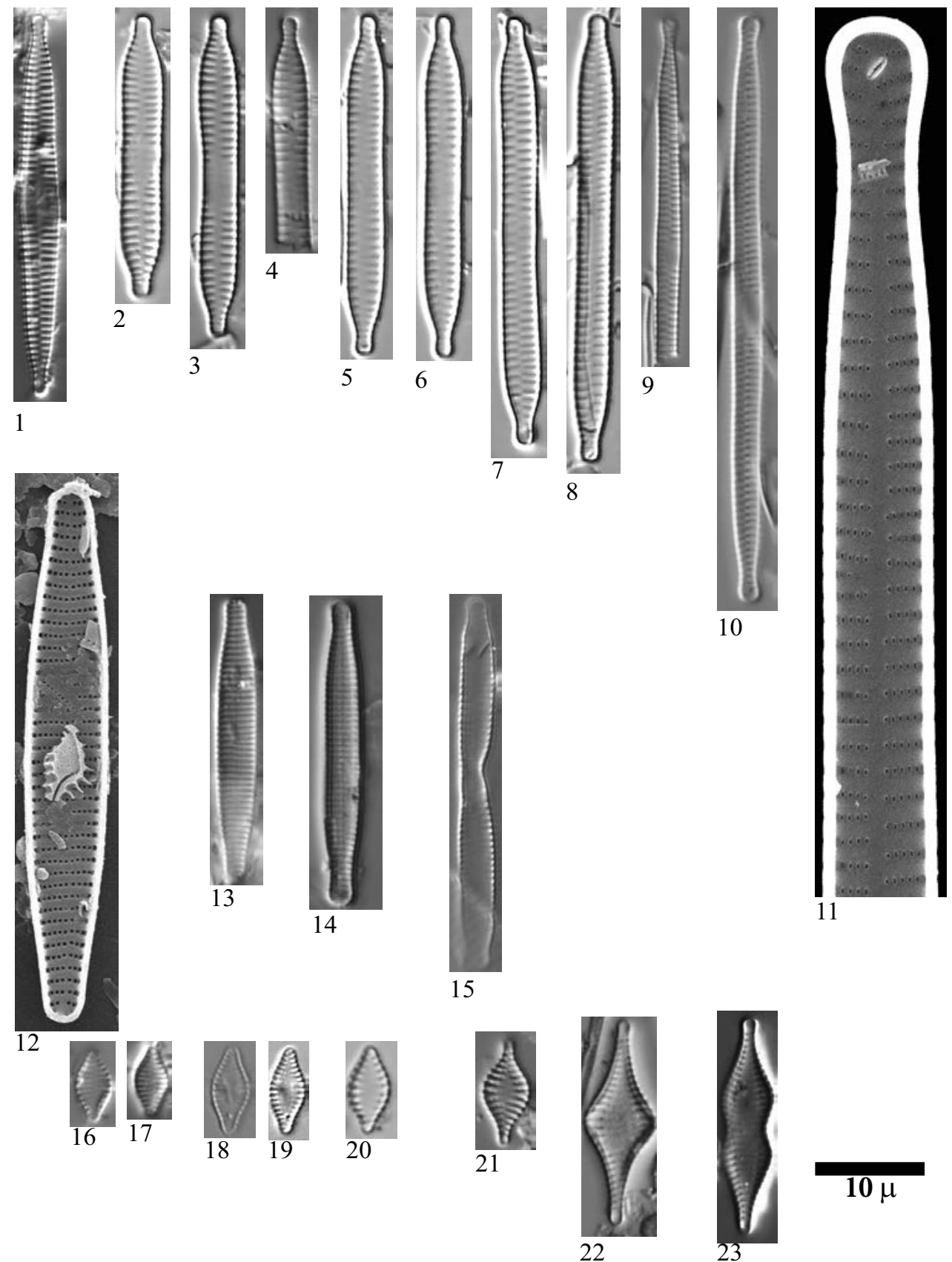
Plate 17 LM: $x 1500$

SEM: Figs. 9-10,13 x10000, Figs.21-22 x6000

Figs. 1-10 Pseudostaurosira microstriata (Marciniak) Flower

Figs. 11-12 Pseudostaurosira cf. brevistriata (Grunow) Williams \& Round

Fig. 13 Pseudostaurosira sp.

Fig. $14 \quad$ Pseudostaurosira sp.

Figs. 15-22 Fragilaria cf. opacolineata Lange-Bertalot

Figs. 1-4, 9-10 Lake Posets, sediment PYR42

Figs. 5-7, 11- Lake Arratille, sediment PYR11

12

Figs. 8, $14 \quad$ Lake Sen, sediment PYR40

Fig. 13 Lake Port Bielh, sediment PYR28

Figs. 15, 17-18 Lake Siscar, sediment PYR126

Fig. 16 Lake Ensangents Superior, sediment PYR106

Fig. 19 Lake Canals Roges, sediment PYR124

Fig. $20 \quad$ Lake Basa de la Mora, sediment PYR32

Figs. 21-22 Lake Argonella de Mes Amunt, sediment PYR78 

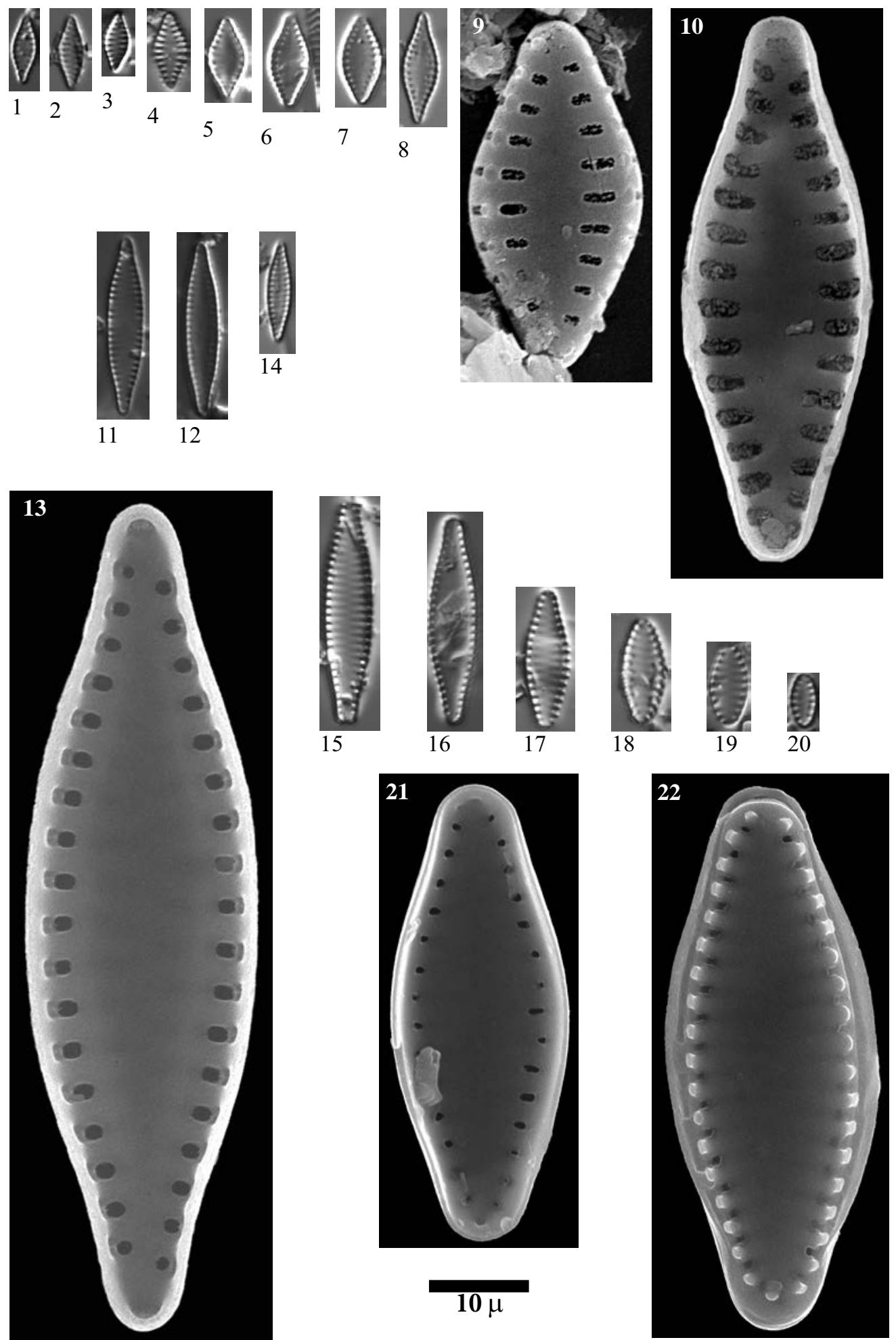
Plate 18

LM: $x 1500$

SEM: $x 6000$

Figs. 1-6

Pseudostaurosira pseudoconstruens (Marciniak) Williams \& Round

Figs. 7-8

Pseudostaurosira cf. robusta (Fusey) Williams \& Round

Figs. 9-11

Pseudostaurosira robusta (Fusey) Williams \& Round

Figs. 12-38

Pseudostaurosira sp. No. 1 Arratille

Figs. 39-41

Pseudostaurosira sp. No. 2 Acherito

Figs. 42-43

Tabularia fasciculata (Agardh) Williams \& Round

Fig. 1

Figs. 2, 11

Figs. 3, 13, 15,

31-32

Figs. 4-6, 12, 14, Lake Arratille, sediment PYR11

16-21, 23-30, $33-35$

Fig. 7

Fig. 8

Fig. 9

Fig. 10

Figs. 22, 37-38, 42

Fig. 36

Figs. 39-41, 43
Lake Burg, sediment

Lake Estom, sediment PYR15

Lake Sen, sediment PYR40

Lake Siscar, sediment PYR126

Lake Posets, sediment PYR42

Lake Burg, sediment BURG1136

Lake Burg, sediment BURG1132

Lake Laurenti, sediment PYR111

Lake Arnales, sediment PYR09

Lake Acherito, sediment PYR01 

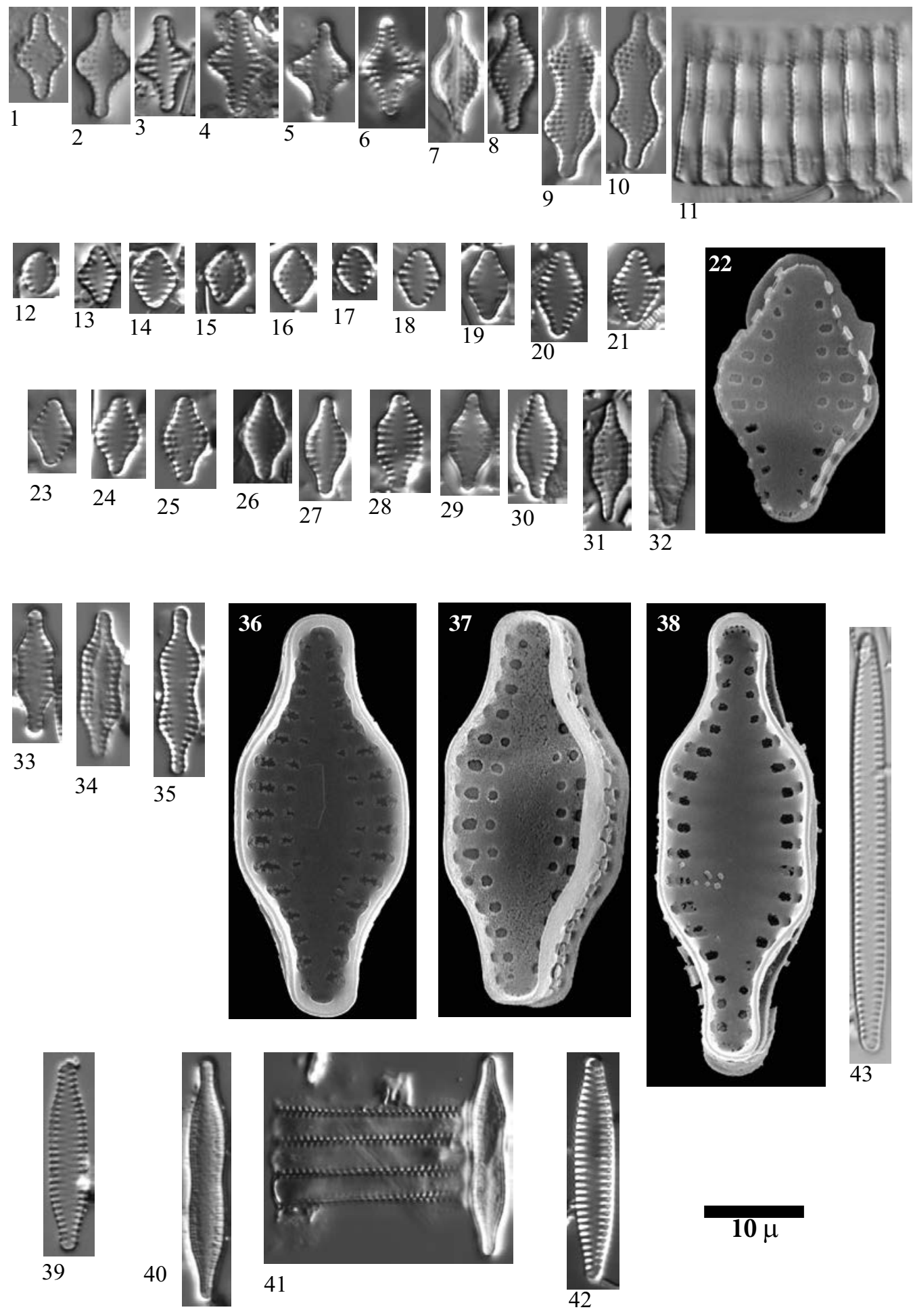
Plate 19 LM: $\mathrm{x} 1500$

SEM: Fig. 3 x 20000, Fig. $4 \times 6000$

Figs. 1-4

Figs. 5-22

Figs. 23-31

Fig. 32

Figs. 33-51

Figs. 52-55

Figs. 56-57
Pseudostaurosira cf. robusta (Fusey) Williams \& Round

Staurosirella pinnata (Ehrenberg) Williams \& Round sensu lato

Staurosirella cf. confusa Morales

Staurosirella oldenburgiana (Hustedt) Morales

Staurosirella pinnata (Ehrenberg) Williams \& Round sensu lato

Punctastriata cf. lancettula (Schumann) Hamilton \& Siver M3

Staurosirella leptostauron (Ehrenberg) Williams \& Round

Figs. 1, 5-8, 19-25 Lake Arratille, sediment PYR11

$28-30,33-36$

$41-46,48-51$

Fig. 2

Lake Posets, sediment PYR42

Figs. 3-4

Lake Roumassot, sediment PYR04

Figs. 9-12, 26-27

Lake Burg, sediment BURG 953

Fig. 13-14

Lake Sen, sediment PYR40

Figs. 15-18

Lake Posets, sediment PYR42

Fig. 31

Lake Burg, sediment BURG 857

Fig. 32

Lake Arnales, sediment PYR09

Figs. 37, 39, 40, 47 Lake Burg, sediment BURG 543 $52-54$

Fig. 38

Lake Burg, sediment BURG 1192

Fig. 55

Lake Burg, sediment BURG 694

Fig. 56

Lake Acherito, sediment PYR01

Fig. 57

Lake Laurenti, sediment PYR111 


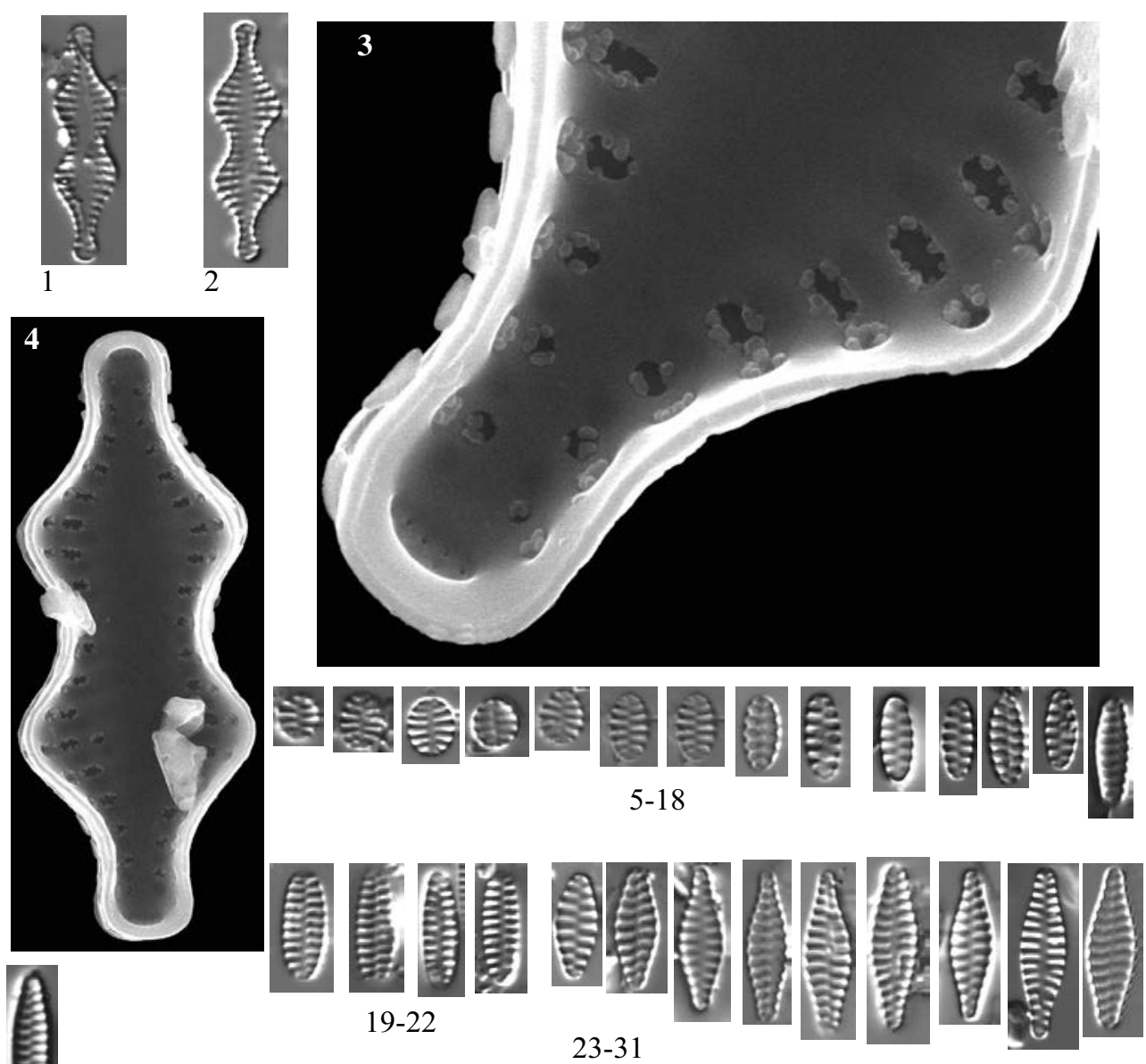

32
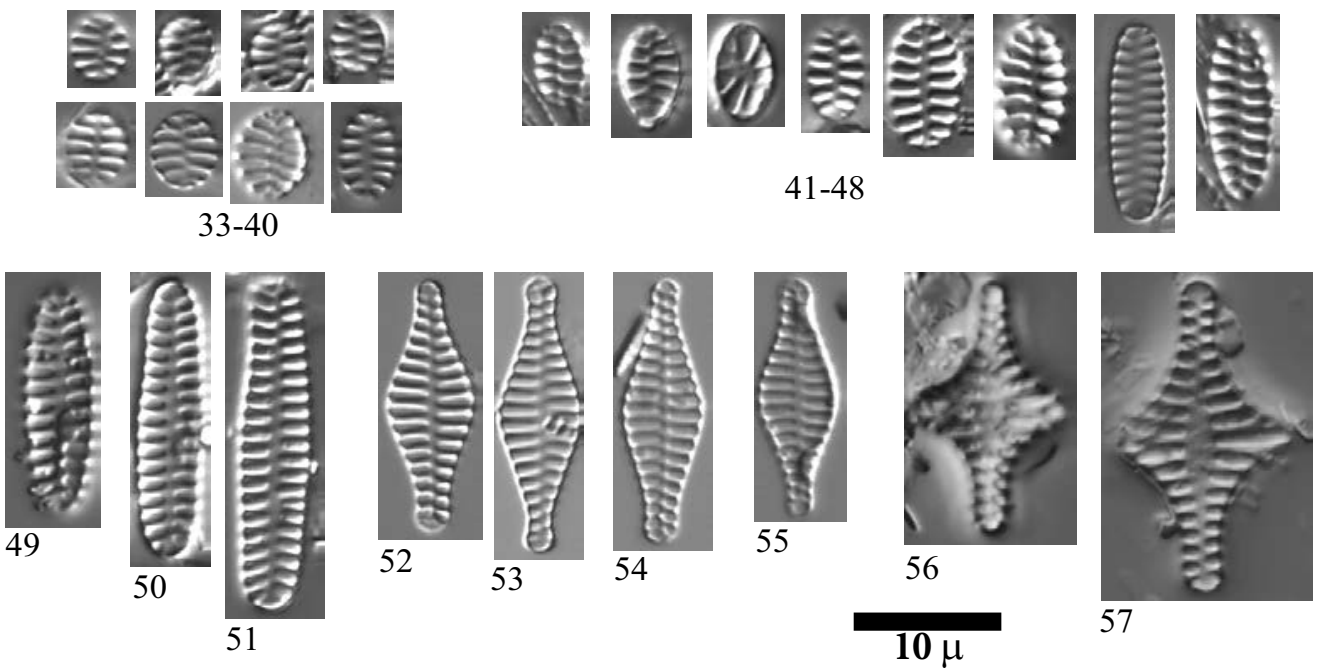
Plate $20 \quad$ LM: $x 1500$

SEM: Figs. 1-5,9 x5000, 7-8 x10000

Figs. 1-4, $7 \quad$ Staurosirella pinnata (Ehrenberg) Williams \& Round

Figs. 5-6 Staurosirella cf. confusa Morales

Figs. 8-9 Punctastriata lancettula (Schumann) Hamilton \& Siver

Fig. 1 Lake Gran de Mainera, sediment PYR70

Figs. 2, 5-6, 9 Lake Laurenti, sediment PYR111

Fig. 3 Lake Gors de Camporrells, sediment PYR110

Fig. 4 Lake Posets, sediment PYR42

Figs. 7-8 Lake Burg, sediment BURG 930 

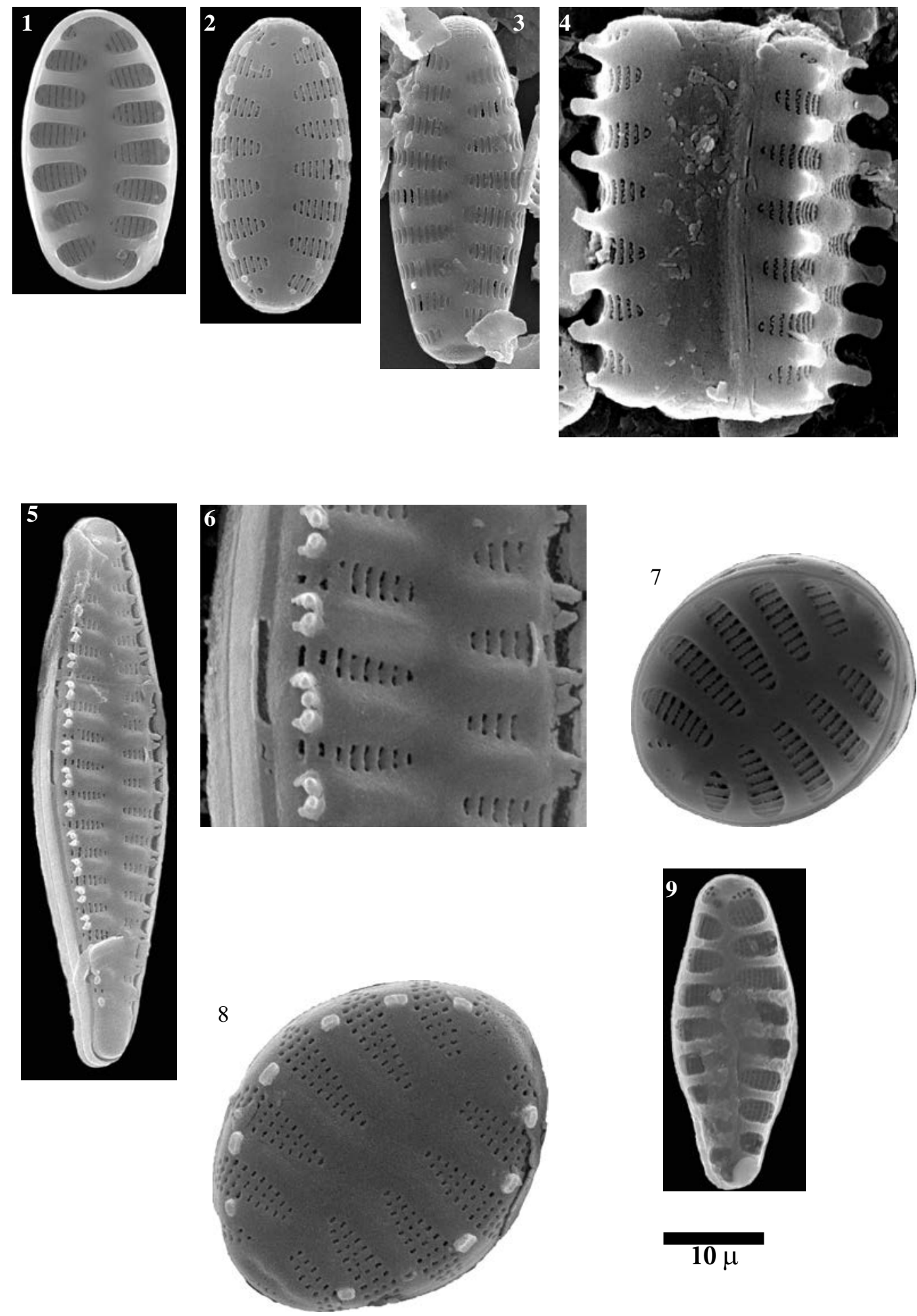
Plate $21 \quad$ LM: Figs. $1.5,7-8 \times 1500$, Fig. $9 \times 750$

SEM: $x 6000$

Figs. 1-6

Figs. 7-9
Hannaea arcus (Ehrenberg) Patrick

Ulnaria biceps (Kützing) Compère sensu lato

Fig. 1 Lake Llebreta sediment PYR58

Figs. 2, 7-9 Lake Arratille, sediment PYR11

Fig. 3

Lake Redon, sediment REDOM

Fig. 4

Lake Les Laquettes 1, sediment PYR27

Fig. 5

Lake Inferior de la Gallina, sediment PYR87

Fig. 6

Lake Laurenti, sediment PYR111 

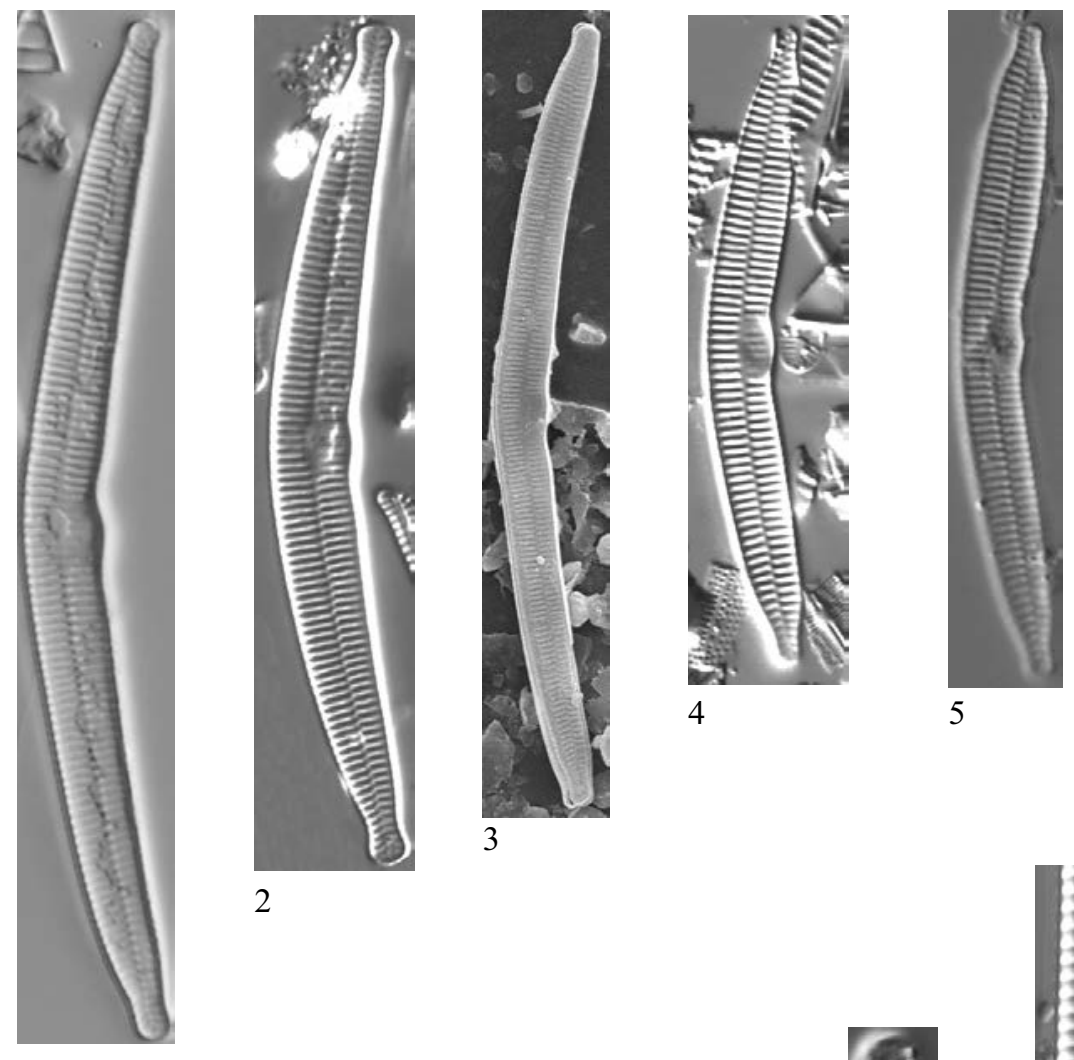

2

1

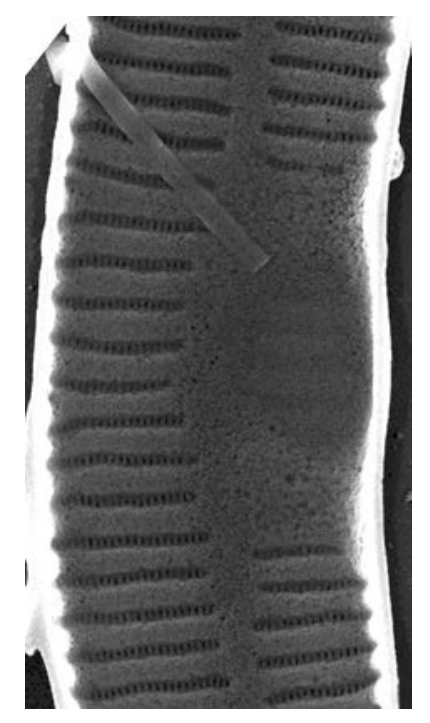

6

$10 \mu$
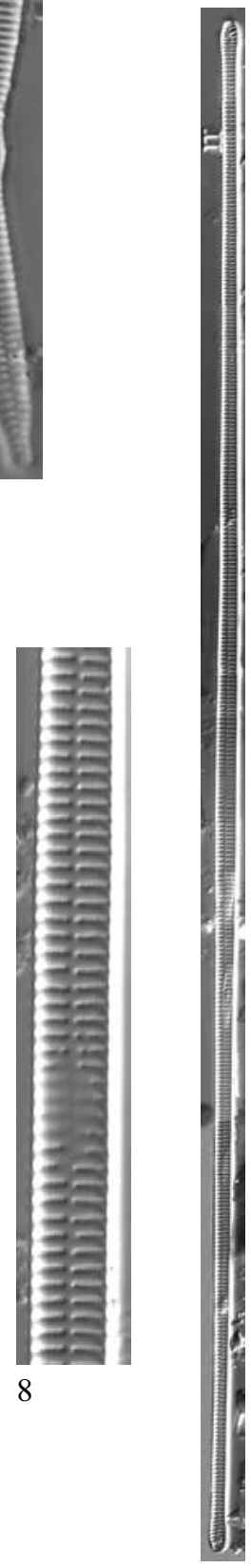
SEM: Figs. $43-47$ x10000, Figs. $48-49$ x8000

Figs. 1-7

Figs. 8-32 $43-48$

Figs. 33-36

Figs. 37-39

Figs. 40-42, 49
Staurosira construens Ehrenberg sensu lato

Staurosira construens var. venter (Ehrenberg) Hamilton

Staurosira construens var. venter (Ehrenberg) Hamilton

Staurosira construens var. binodis (Ehrenberg) Hamilton

Staurosira construens aff. var. venter (Ehrenberg) Hamilton

Figs. 1-2, 4-7, Lake Burg, sediment BURG 543 8,10

Figs. 3, 22, Lake Burg, sediment BURG 1136 31-32

Fig. 11

Lake Burg, sediment BURG 853

Fig. 12

Lake Burg, sediment BURG 844

Figs. 13-14 Lake Burg, sediment BURG 831

Fig. 15

Lake Estom, sediment PYR15

Figs. 16-17

Lake Sen, sediment PYR40

Figs. 19-24, 30

Lake Arratille, sediment PYR11

Figs. 26-29, 39 Lake Ormiélas, sediment PYR05

Figs. 33-35 Lake Burg, sediment BURG 513

Fig. 36 Lake Les Laquettes 1, sediment PYR27

Fig. 37 Lake Burg, sediment BURG 420

Fig. 38 Lake Burg, sediment BURG 1181

Fig. 39 Lake Asnos, sediment PYR14

Figs. 41-42 Lake Acherito, sediment PYR01

Figs. 43-47 Lake Burg, sediment BURG 930

Fig. 48

Lake Mariola, sediment PYR80

Fig. 49 Lake Port Bielh, sediment PYR28 

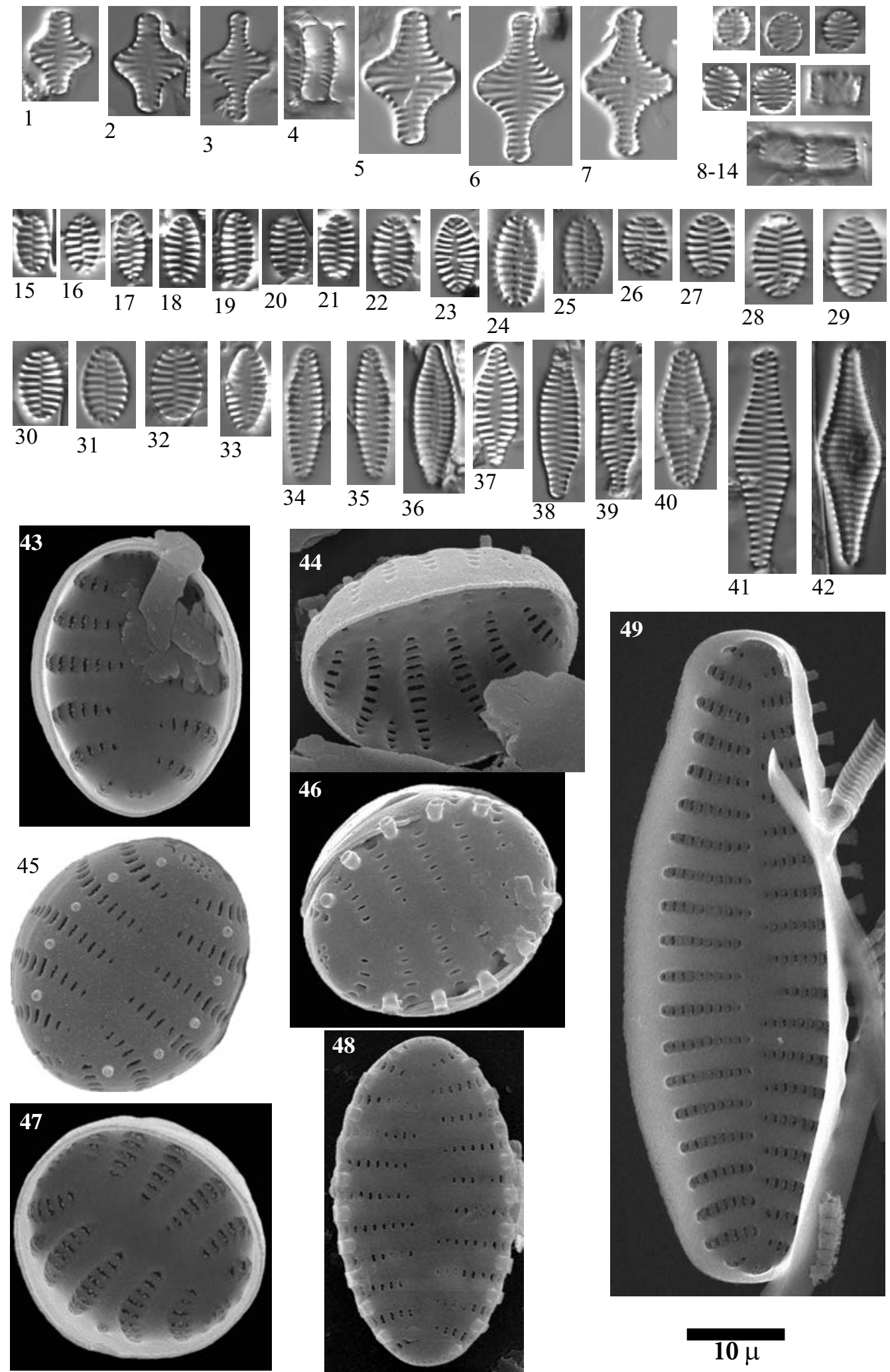

Limnetica, 36 (1): 127-395 (2017) 
Plate $23 \quad$ LM: $x 1500$

SEM: $\mathrm{x} 7500$

Figs. 1-13 Eunotia palatina Lange-Bertalot \& Krüger

Fig. 14 Eunotia cf. palatina Lange-Bertalot \& Krüger

Fig. 1 Lake Airoto, sediment PYR73

Figs. 2-13 Lake Pica, sediment PYR100

Fig. 14 Lake Monges, sediment PYR57

Fig. 13 Manfred Ruppel photo 

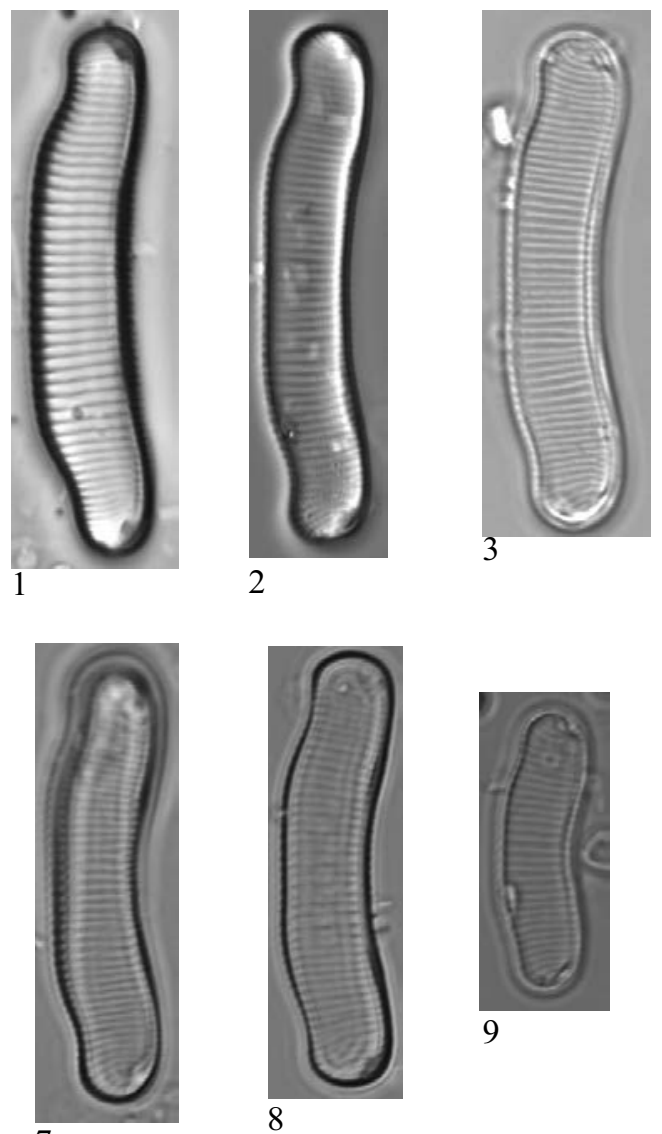

7
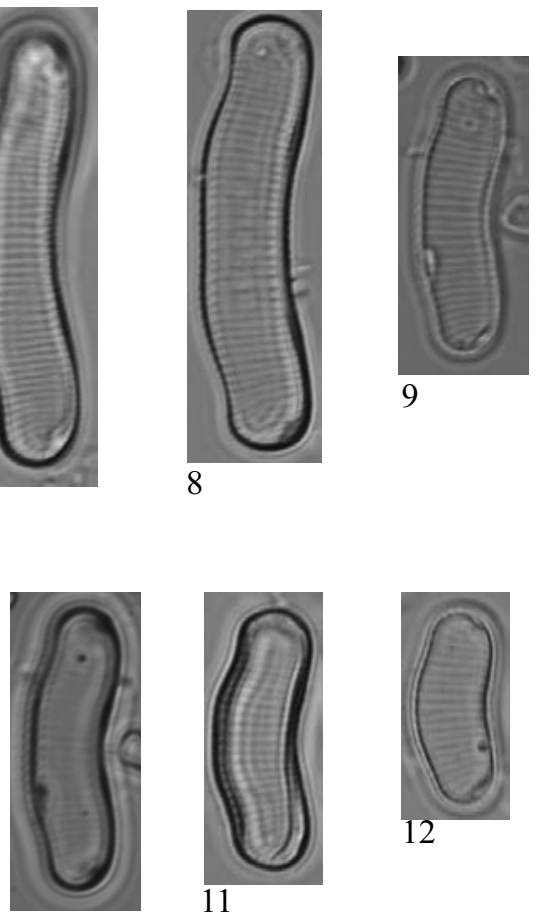

10
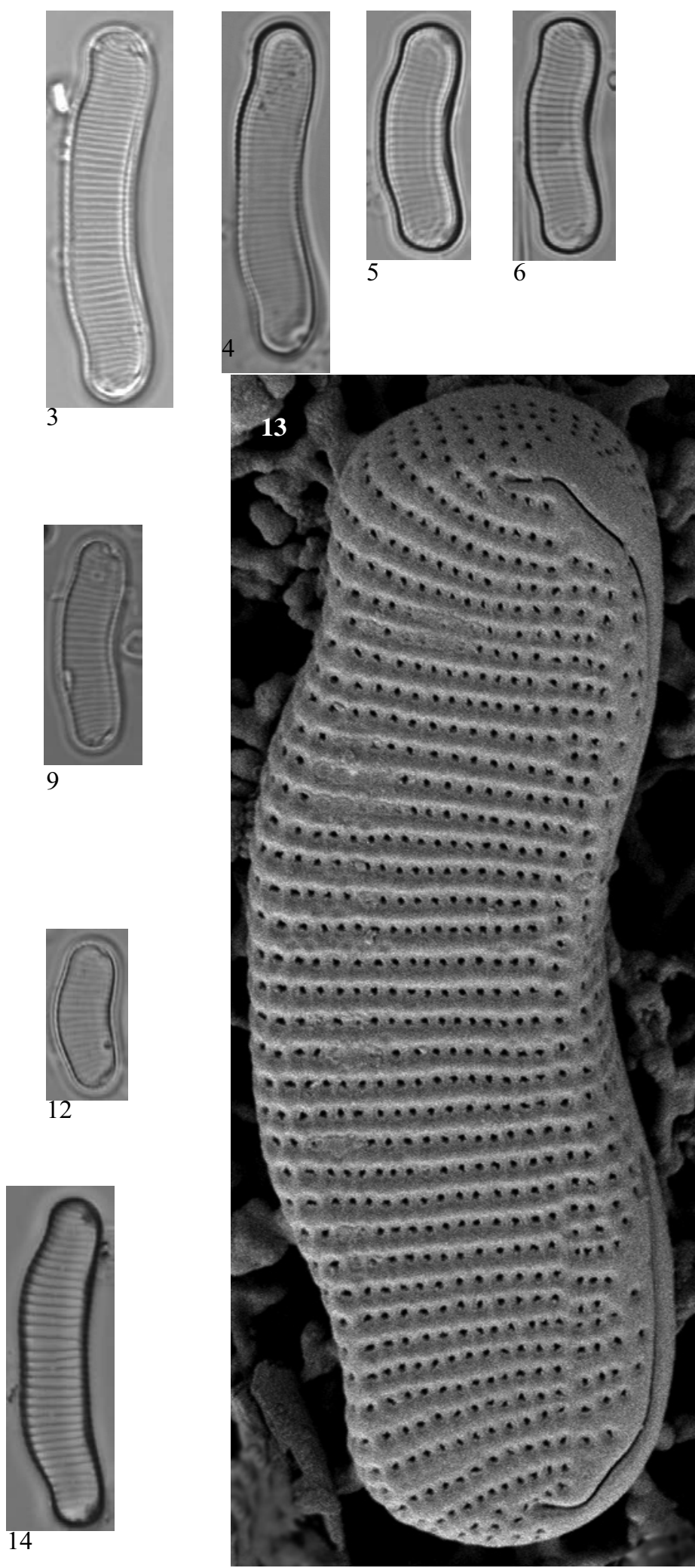

Limnetica, 36 (1): 127-395 (2017) 
Plate $24 \quad$ LM: $x 1500$

SEM: Figs. 4-5 x2000, Fig. 22 x6000, Fig. 25 x9000, Figs. 23-24, $26-27 \times 10000$

Figs. 1-11 Eunotia catalana Lange-Bertalot \& Rivera-Rondón

Fig. 12

Eunotia lapponica Grunow ex Cleve

Fig. $1 \quad$ Lake Sotllo, epilithic EpiPYR89

Figs. 2-4, 9-12 Lake Baiau Superior, sediment PYR76

Figs. 5-6 Lake Negre, sediment PYR79

Figs. 7-8 Lake Pica Palòmera, sediment PYR52

Figs. 9-11 Manfred Ruppel photos 

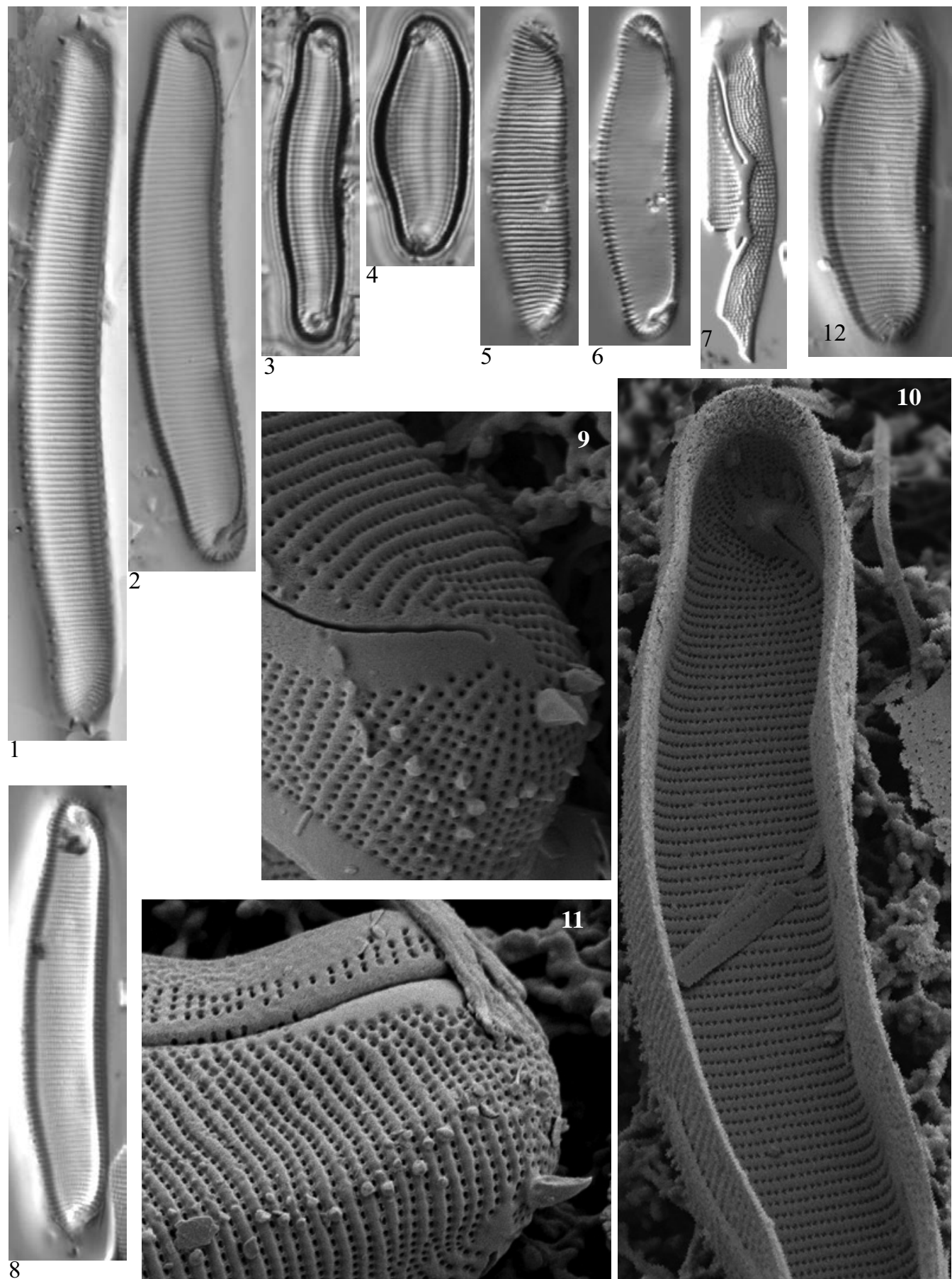

ร:ก:

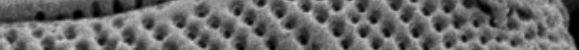

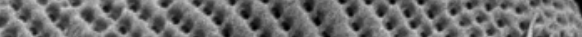

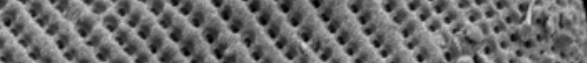

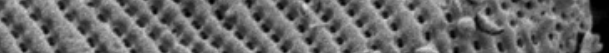

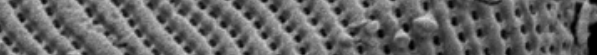

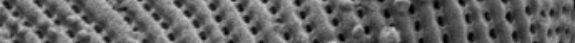

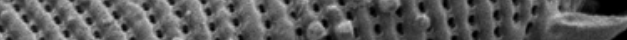

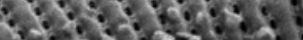

Hon:

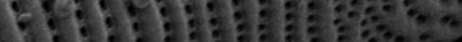

$2: 2: 25: 25 \%$

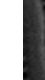

$10 \mu$
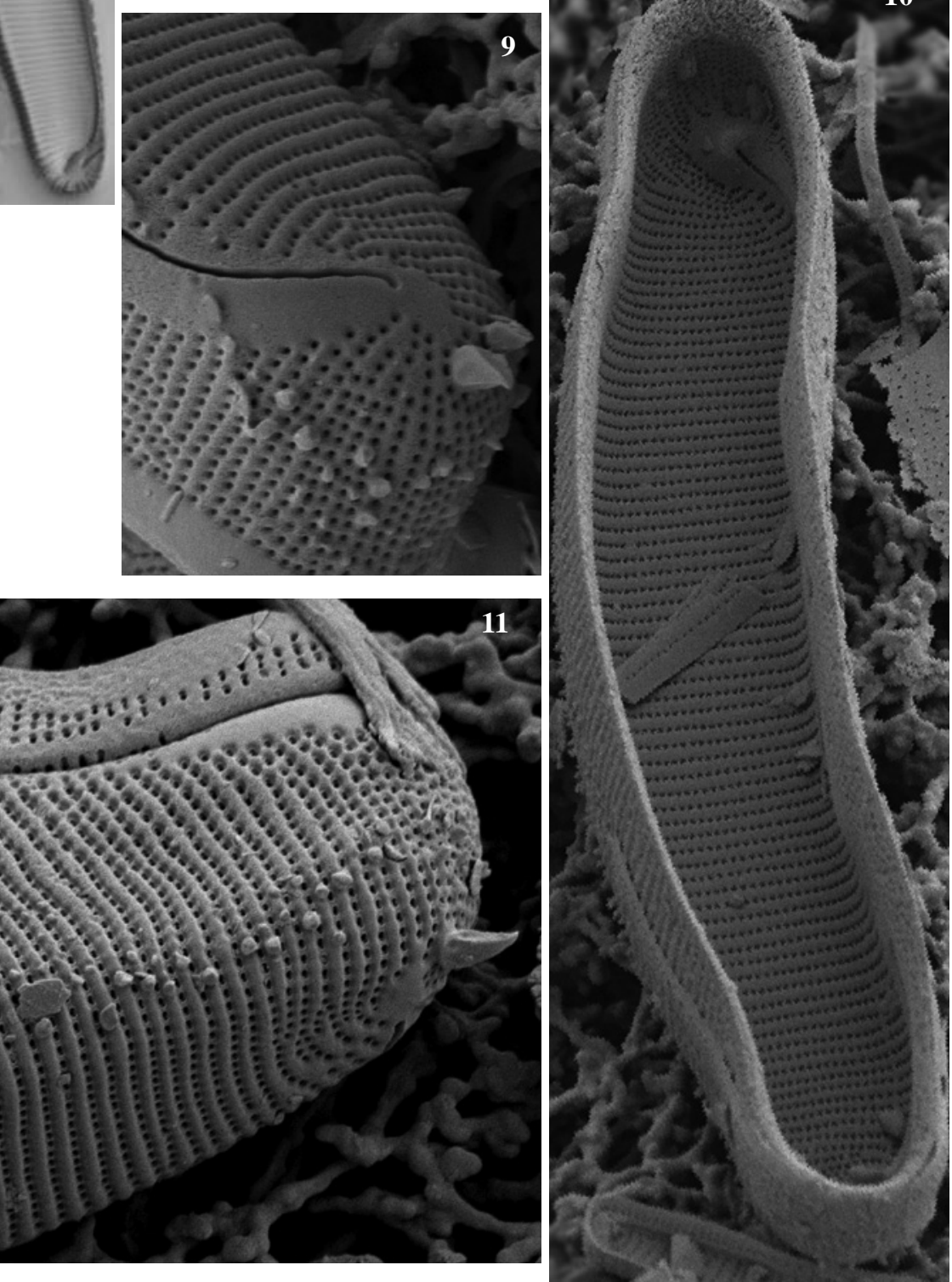

Limnetica, 36 (1): 127-395 (2017) 
LM: $x 1500$

SEM $\times 3500$

Fig. 1

Fig. 2-3

Fig. 4

Figs. 5-6

Figs. 7-9

Figs. 10-11

Fig. 12

Figs. 13-14

Fig. 15

Figs. 16-17

Figs. 18-19

Fig. 1

Fig. 2

Fig. 3

Fig. 4

Figs. 5-6

Fig. 7

Fig. 8

Figs. 9-11

Fig. 12

Figs. 13-15

Fig. 16

Fig. 17

Figs. 18-19
Eunotia suecica A. Cleve

Eunotia diadema Ehrenberg

Eunotia praerupta Ehrenberg

?Eunotia circumborealis Lange-Bertalot \& Nörpel

Eunotia cf. dorofeyukae Lange-Bertalot \& Kulikovskiy

Eunotia cf. circumborealis Lange-Bertalot \& Nörpel

Eunotia aff. minor (Kützing) Grunow

Eunotia curtagrunowii Nörpel-Schempp \& Lange-Bertalot

?Eunotia meridionalis Lange-Bertalot \& Tagliaventi

? Eunotia islandica Østrup

Eunotia cisalpina Lange-Bertalot \& Cantonati

Eunotia sp.

Lake Forcat Inferior, epilithic PYR77

Lake Baiau Superior, sediment PYR69

Lake Redon, sediment REDOM

Lake Estelat, sediment PYR120

Lake Mariola, sediment PYR80

Lake Burg, sediment BURG830

Lake Acherito, epilithic EpiPYR01

Lake Acherito, sediment PYR01

Lake Burg, sediment BURG506

Lake Senó, sediment PYR84

Lake Monges, sediment PYR57

Lake Llosás, sediment PYR46

Lake PYR127, sediment sample 

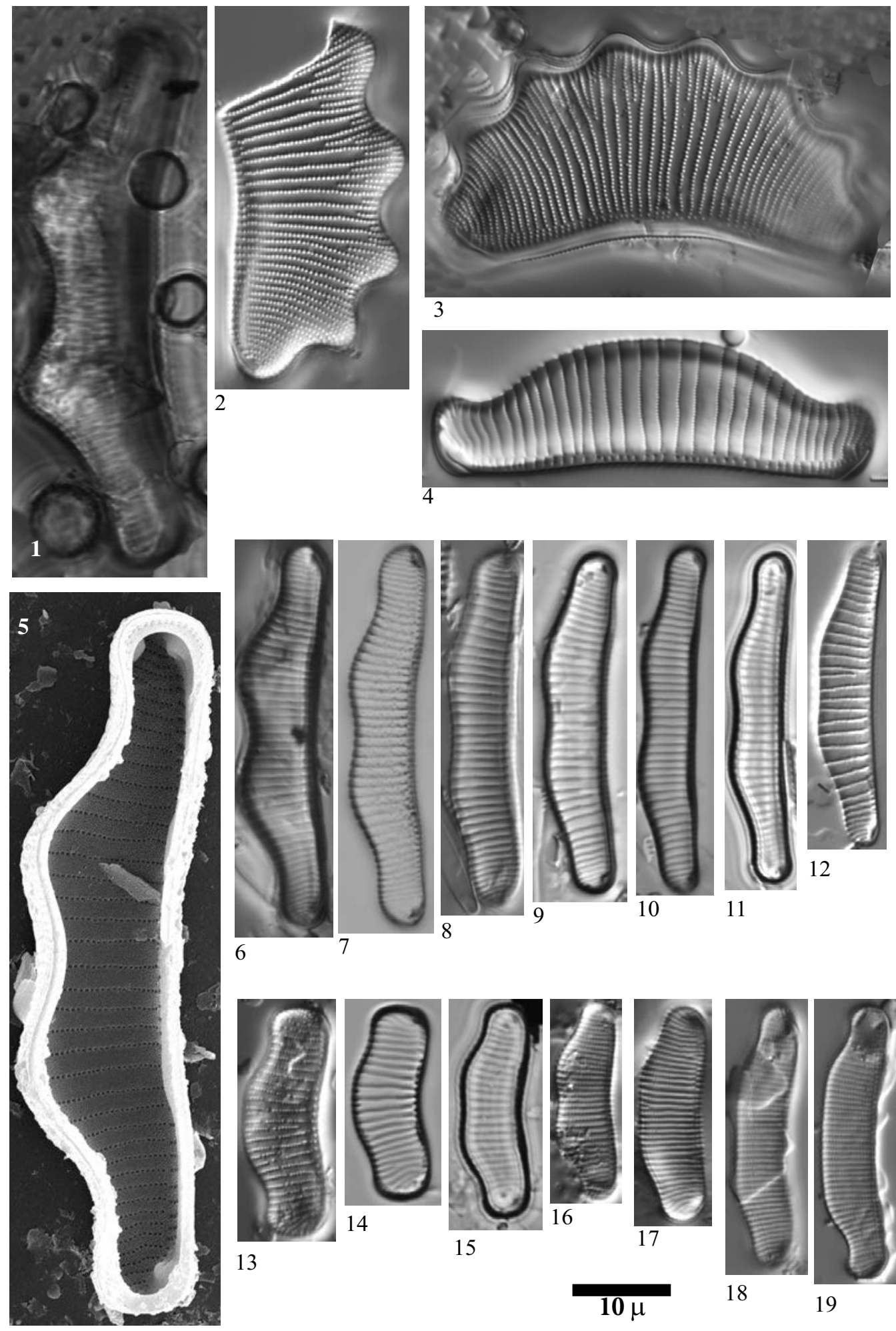

13
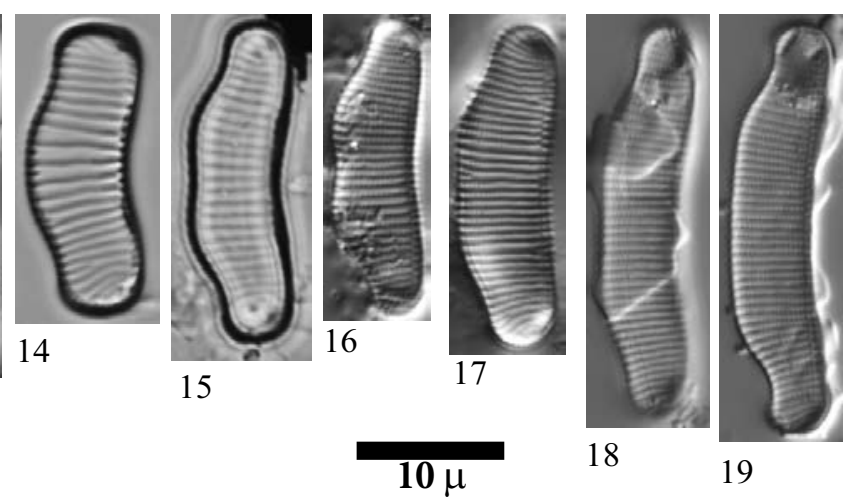
Plate 26

LM: $x 1500$

Figs. 1-3

Figs. 4-5

Fig. 6

Fig. 7

Fig. 8

Figs. 1, 6

Fig. 2

Fig. 3

Fig. 4

Fig. 5

Fig. 7

Fig. 8
Eunotia glacialis Meister

Eunotia valida Hustedt

Eunotia minor (Kützing) Grunow

?Eunotia minor. primary cell?

Eunotia pectinalis (Kützing) Rabenhorst
Lake Mariola, sediment PYR80

Lake Illa, sediment PYR66

Lake Senó, sediment PYR84

Lake Blaou, epilithic EpiPYR94

Lake Angonella, sediment PYR78

Lake Long de Liat, sediment PYR55

Lake Blaou, sediment PYR94 

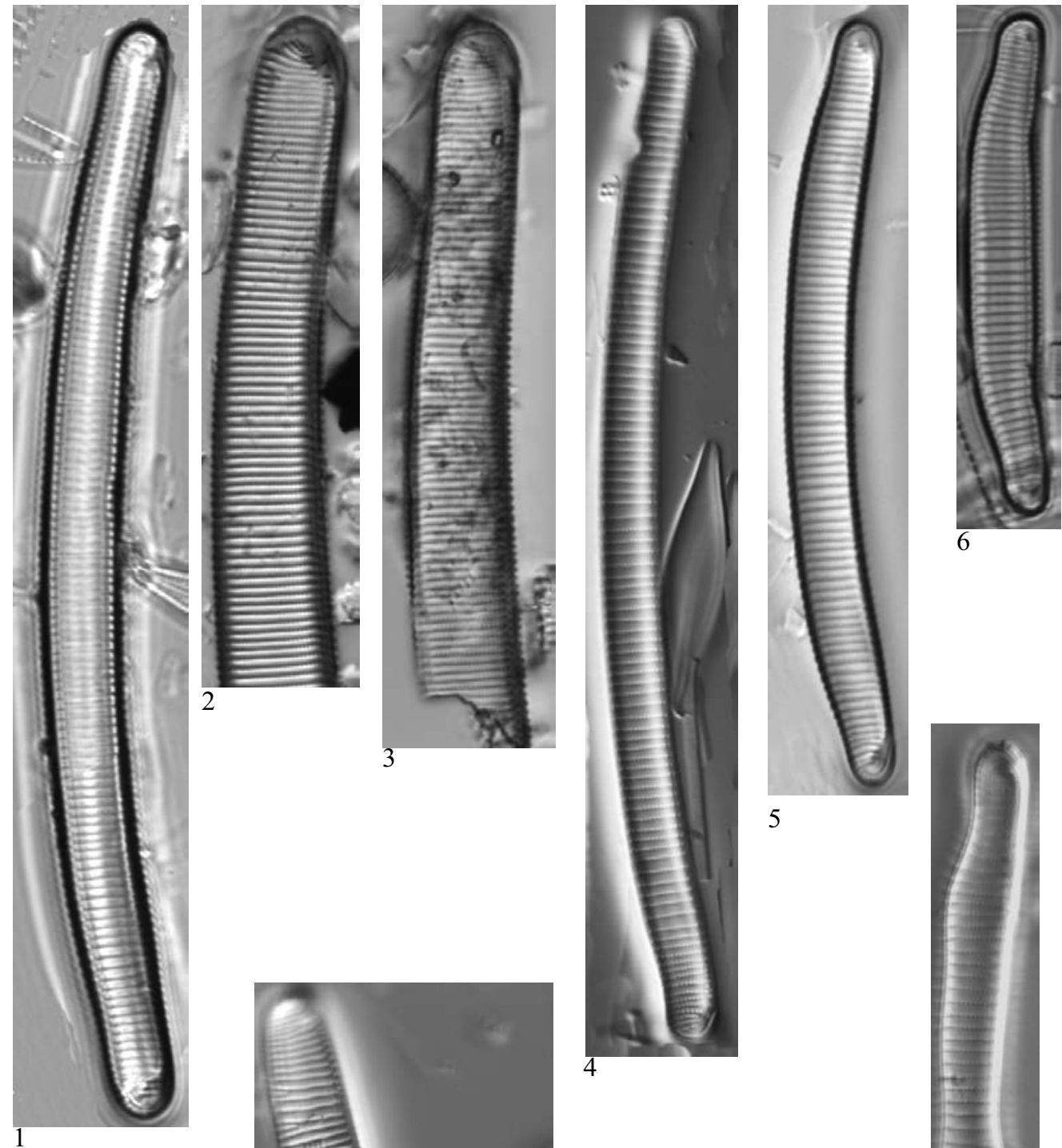

5

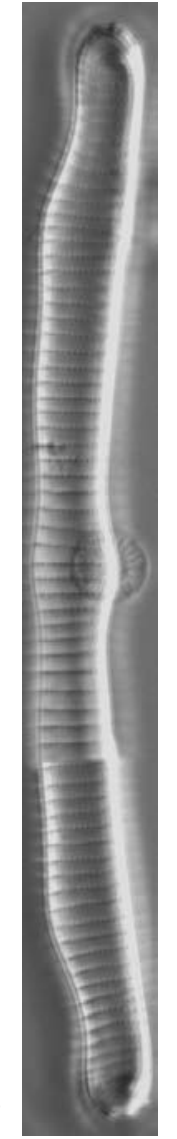

Limnetica, 36 (1): 127-395 (2017) 
Plate 27 LM: $x 1500$

SEM: Figs. 4 x2000, Figs. $5-6$ x3000, Fig. 7 x8000

Eunotia arcus Ehrenberg sensu lato

Fig. 1 Lake Port Bielh, sediment PYR28

Fig. 2 Lake Senó, epilithic EpiPYR84

Figs. 3-4 Lake Redon, sediment REDOM

Fig. 5 Lake Laurenti, sediment PYR111

Figs. 6-7 Lake Angonella, epilithic EpiPYR78 

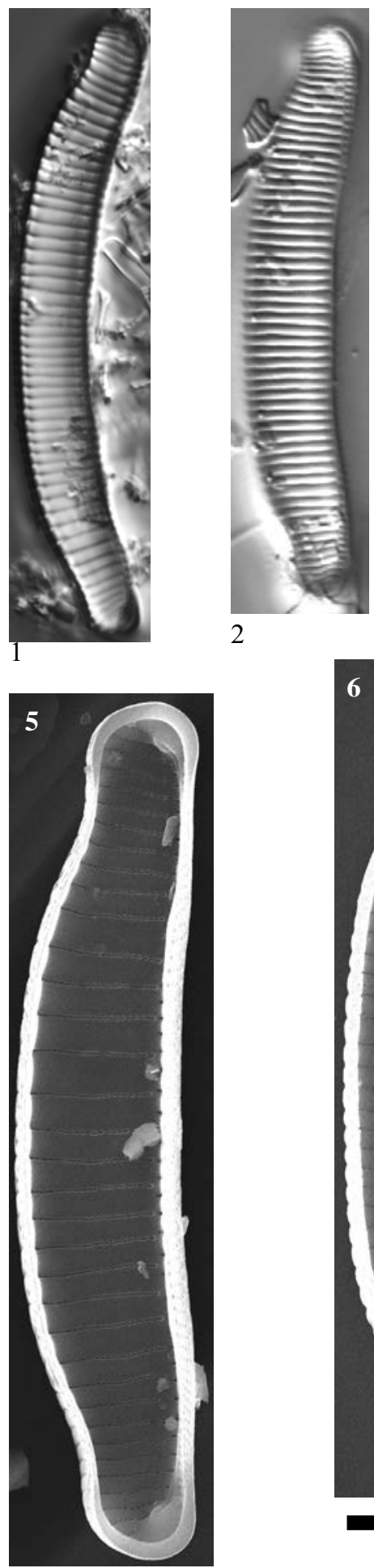

$$
6
$$

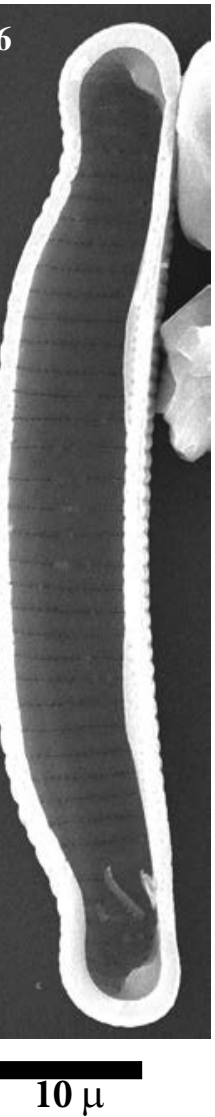

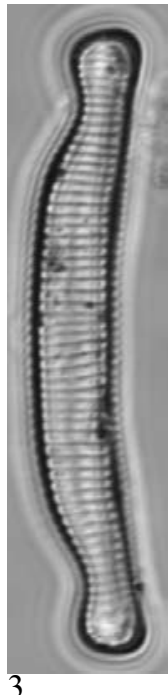

4
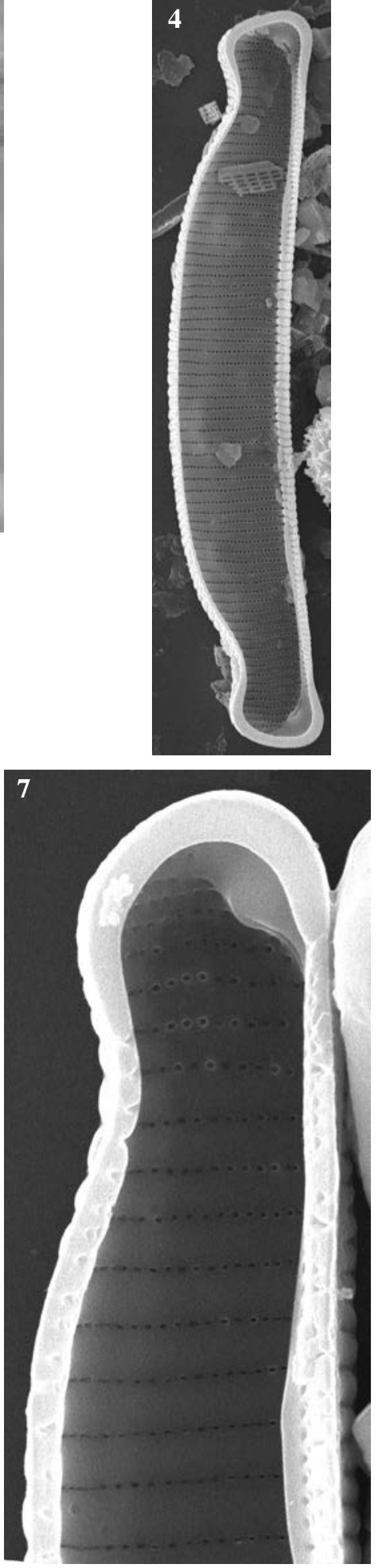
LM: $x 1500$

SEM: $x 6000$

Figs. 1-3 Eunotia aff. soleirolii (Kützing) Rabenhorst

Figs. 4-5 Eunotia novaisiae Lange-Bertalot \& Luc Ector

Figs. 6-9 Eunotia aff. soleirolii (Kützing) Rabenhorst

Fig. $10 \quad$ Eunotia sudetica O. Müller

Figs. 11-12 Eunotia boreoalpina Lange-Bertalot \& Nörpel-Schempp

Figs. 13-17 Eunotia incisa Gregory

Fig. $18 \quad$ Eunotia cf. faba Ehrenberg

Fig. $19 \quad$ Eunotia sp

Figs. 20-22 Eunotia intermedia (Krasske) Nörpel \& Lange-Bertalot

Figs. 23-25 Eunotia cf. implicata Norpel, Alles \& Lange-Bertalot

Figs. 26-29 Peronia fibula (Brébisson in Kützing) Ross

Figs. 1-2 Lake Gelat Bergús, sediment PYR65

Figs. 3-5 Lake Monges, sediment PYR57

Fig. 6 Lake Mariola, sediment PYR80

Figs. 7-9 Lake Illa, sediment PYR66

Fig. $10 \quad$ Lake Negre, sediment PYR79

Figs. 11, 16-17, Lake Senó, sediment PYR84

24-26

Figs. 12, 27 Lake Inferior de la Gallina, sediment PYR87

Figs. 13, $20 \quad$ Lake Romedo de Dalt, sediment PYR85

Figs. 14, 15 Lake Aixeus, epilithic PYR92

Figs. 18-19, 27 Lake Senó, epilithic EpiPYR84

Figs. 21, $23 \quad$ Lake Sotllo, sediment PYR89

Fig. 22 Lake Baiau superior, sediment PYR76 

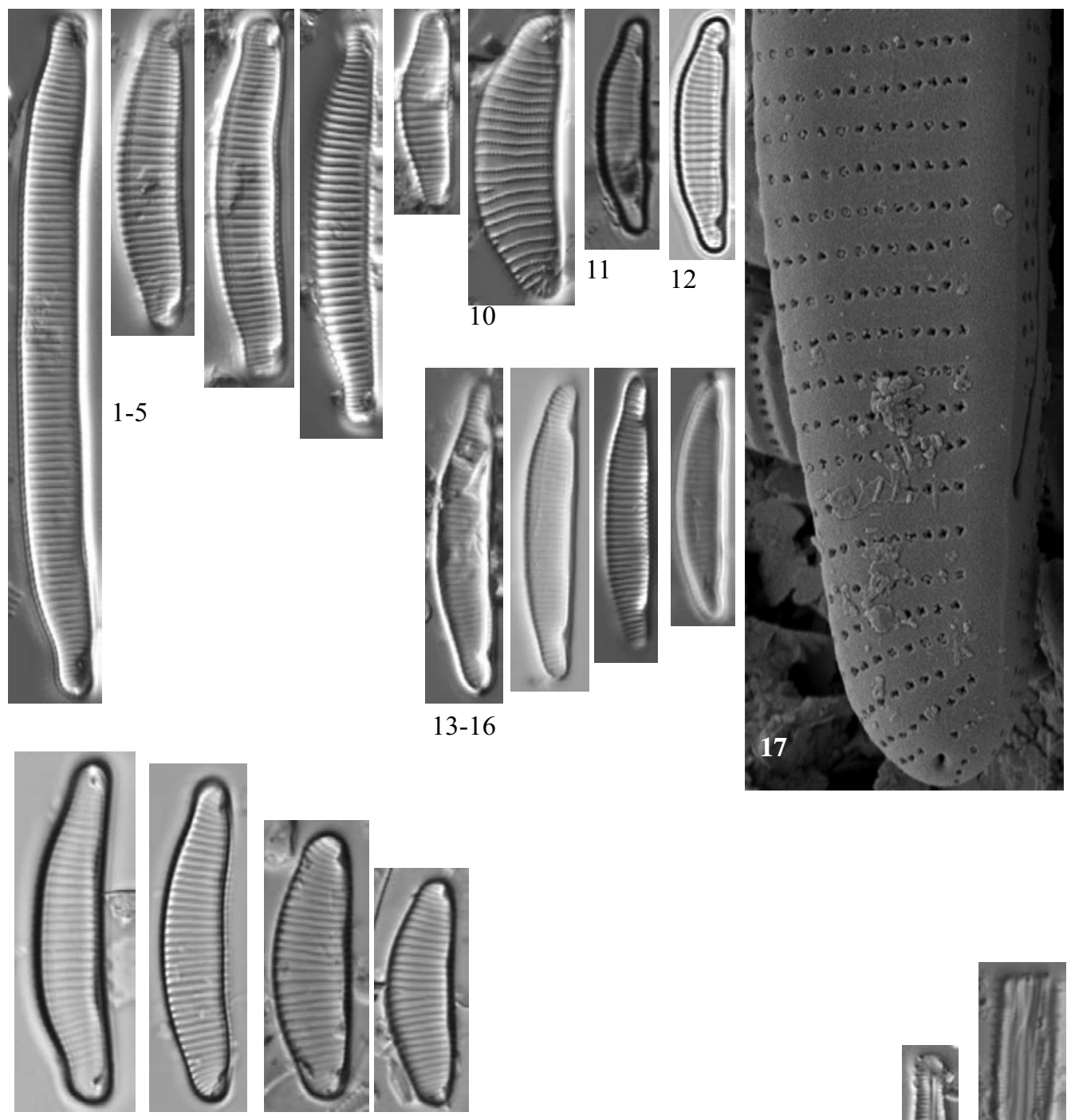

13-16

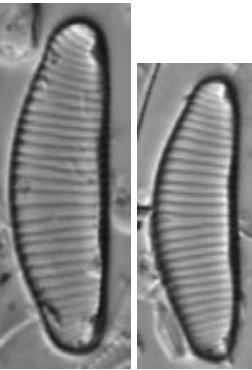

6-9
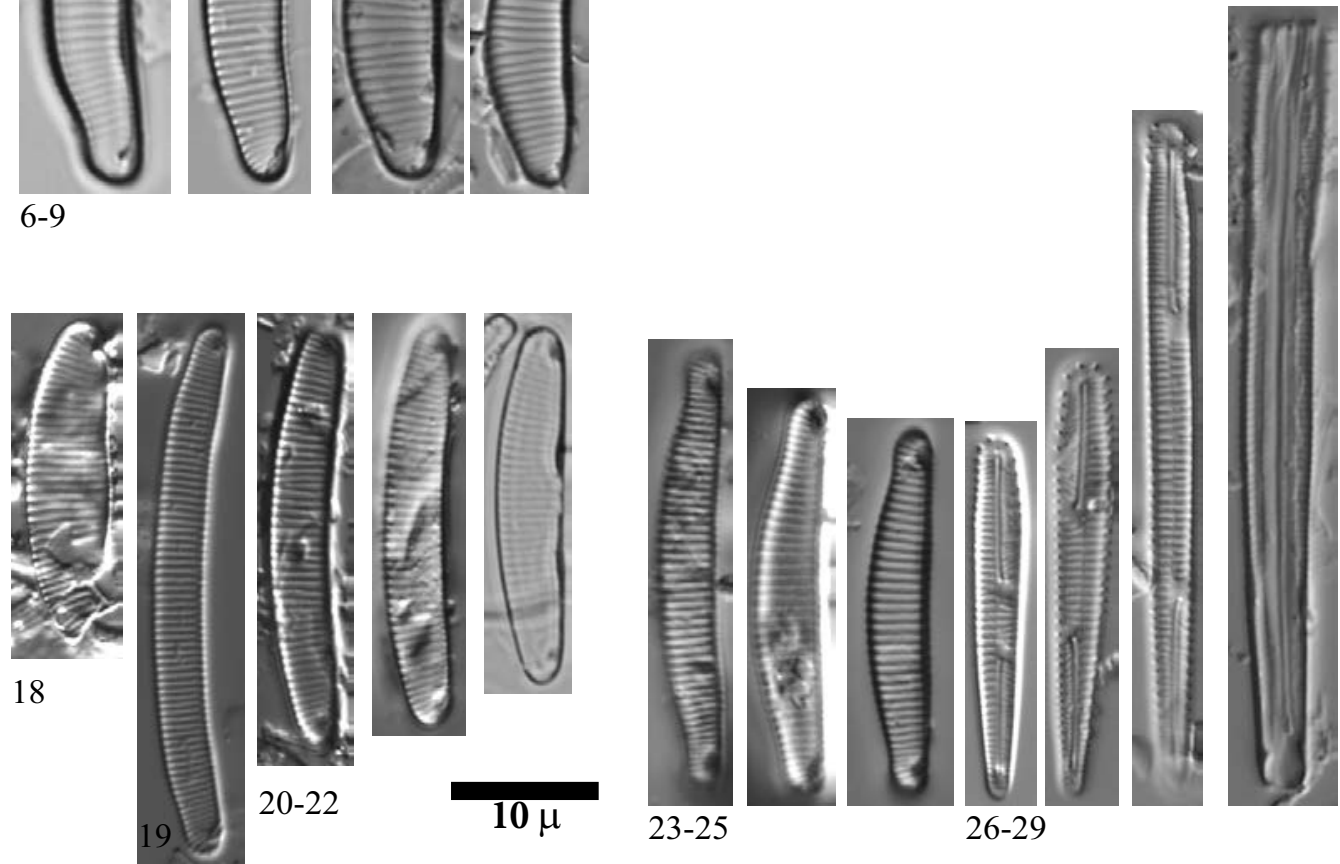

Limnetica, 36 (1): 127-395 (2017) 
Plate 29 SEM: $\mathrm{x} 6000$

Fig. 1 Eunotia intermedia (Krasske) Nörpel \& Lange-Bertalot

Fig. 2

Eunotia cf. botuliformis Wild, Nörpel \& Lange-Bertalot

Figs. 3-5

Eunotia minor (Kützing) Grunow sensu lato

Figs. 1, 5 Lake Pica Palòmera, sediment PYR52

Fig. 2 Lake Illa, sediment PYR66

Figs. 3-4 Lake Senó, sediment PYR84

Figs. 1-5 Manfred Ruppel photos 

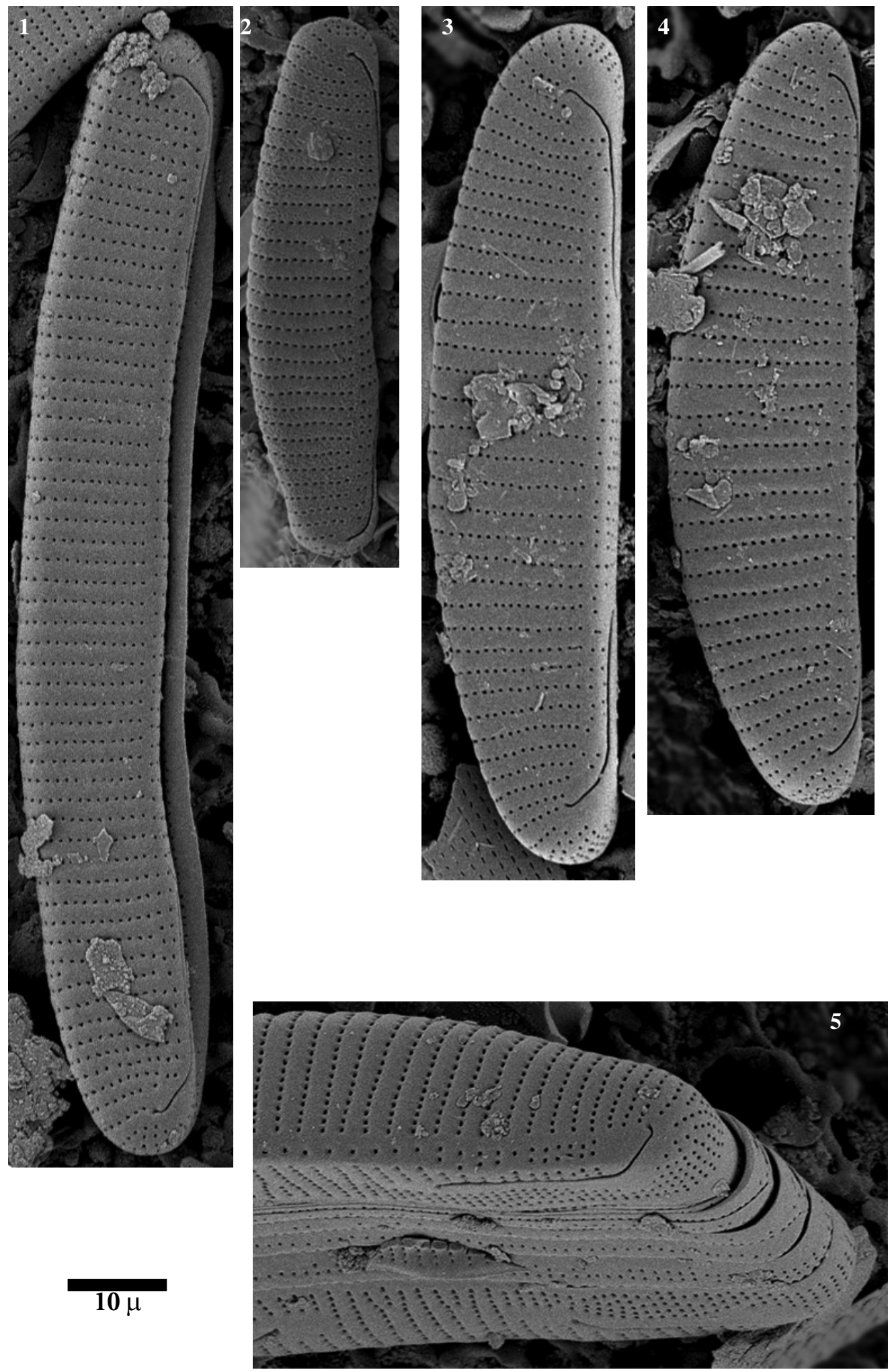

Limnetica, 36 (1): 127-395 (2017) 
LM: x1500

Figs. 1-10

Fig. 11

Figs. 12-39
Eunotia novaisiae Lange-Bertalot \& Luc Ector

Eunotia cf. implicata Norpel, Alles \& Lange-Bertalot

Eunotia novaisiae var. altopyrenaica Lange-Bertalot \& Rivera -Rondón

Figs. 1, 3-8, 10, 31, 37 Lake Senó, sediment PYR84 39

Figs. 2, 9, 15, 24, 27, Lake Pica Palòmera, sediment PYR52 34

Fig. 11

Figs. 12-14, 17-23

Lake Sotllo, sediment PYR89

Figs. 16, 26, 29-30, Lake Baiau superior, sediment PYR76 $32-22,35-36,38$

Fig. 25

Lake Illa, sediment PYR66

Figs. 28

Lake Mariola, sediment PYR80

Lake Aixeus, sediment PYR92 

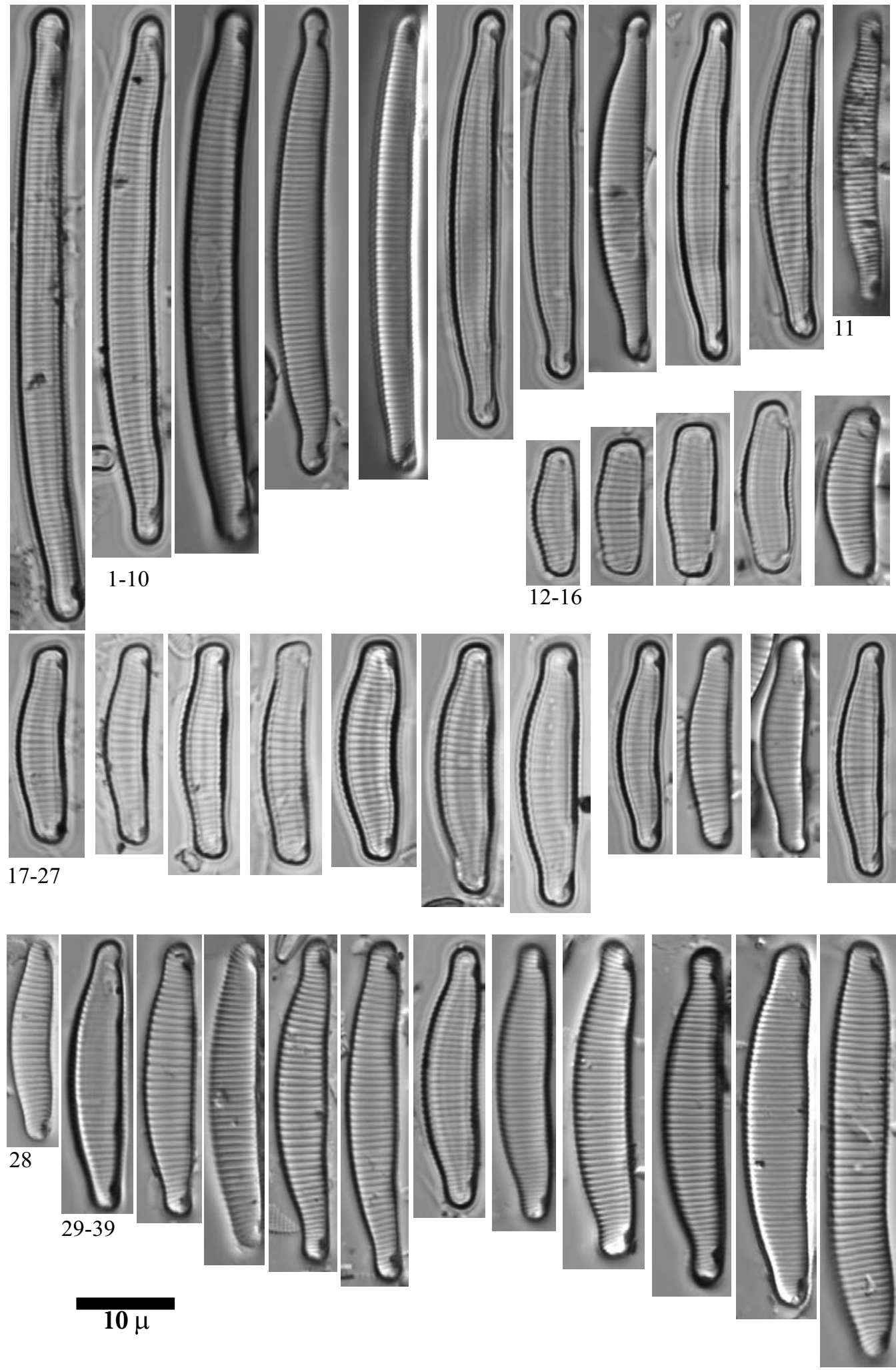
LM: x1500

Figs. 1-16, 26-29

Figs. 17-22, 24-25

Fig. 23

Figs. 30-31

Figs. 32-71

Figs. 72-77

Figs. 1-2, 5-7, 9-16 $27,29,70-71,77$

Figs. 3-4, 17-18, 26, 28, 64-65, 76

Fig. 8

Figs. 19-22, 61-63, 74-75

Figs. 23, 45-48

Fig. 24, 31, 49-60, 72-73

Figs. 25, 32-44

Fig. 30

Figs. 66-67

Figs. 68-69
Eunotia subarcuatoides Alles, Norpel \& Lange-Bertalot

Eunotia cf. seminulum Norpel-Schempp \& Lange-Bertalot

Eunotia seminulum Norpel-Schempp \& Lange-Bertalot

Eunotia cf. intermedia (Krasske) Nörpel \& Lange-Bertalot

Eunotia sp

aff. E. pseudogroenlandica Lange-Bertalot \& Tagliaventi aff. E. botuliformis Wild, Nörpel \& Lange-Bertalot

Eunotia spp.

Lake Pica Palòmera, epilithic EpiPYR52

Lake Nere de Güèri, epilithic EpiPYR53
Lake Garbet, sediment PYR81

Lake Aixeus, epilithic EpiPYR92

Lake Negre, sediment PYR79

Lake Sotllo, sediment PYR89

Lake Baiau superior, sediment PYR76

Lake Les Laquettes, sediment PYR27

Lake Senó, sediment PYR84

Lake Pica Palòmera, sediment PYR52 

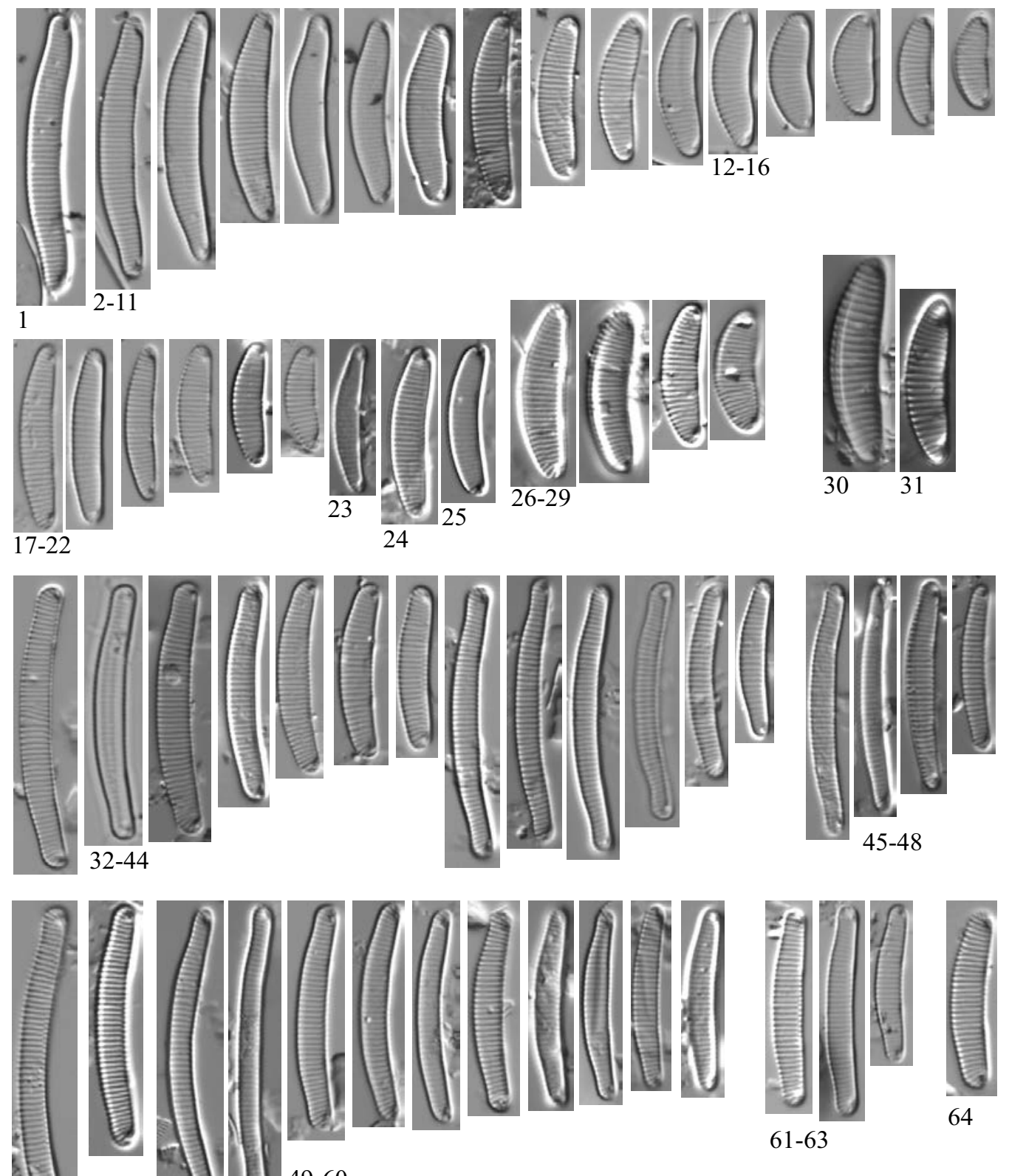
$49-60$
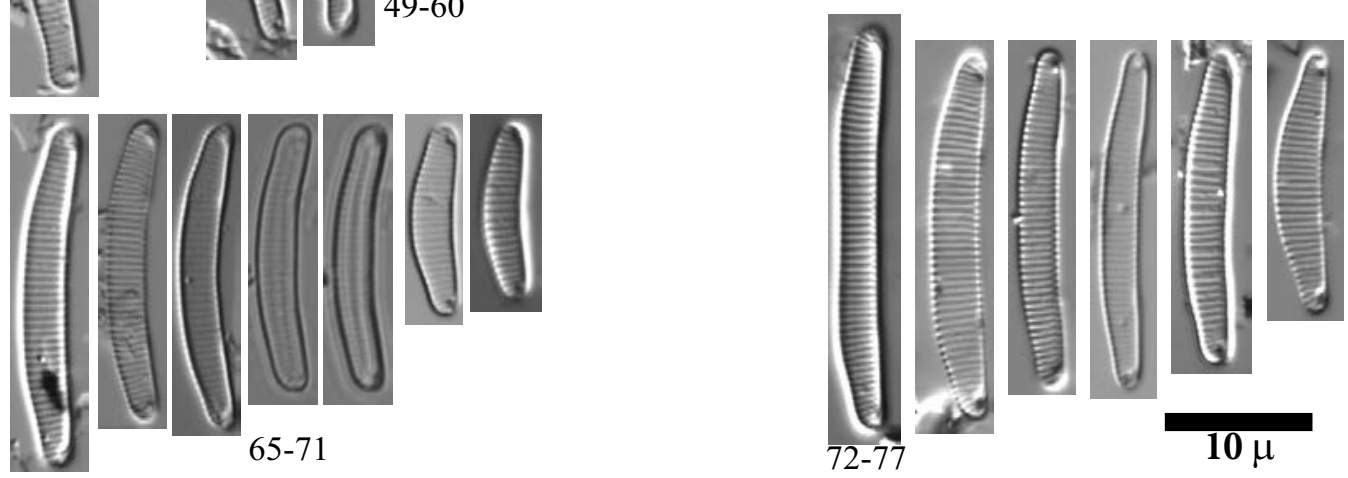

Limnetica, 36 (1): 127-395 (2017) 
SEM: Fig. 3 x 20000, Fig. 4x6000

Figs. 1-2

Eunotia novaisiae var. altopyrenaica Lange-Bertalot \& RiveraRondón

Figs. 3-4

Eunotia sp

aff. E. pseudogroenlandica Lange-Bertalot \& Tagliaventi

aff. E. botuliformis Wild, Nörpel \& Lange-Bertalot

Figs. 5-6

Eunotia subarcuatoides Alles, Norpel \& Lange-Bertalot

Figs. 1, 4, 6 Lake Senó, sediment PYR84

Fig. 2

Lake Baiau superior, sediment PYR76

Fig. 3

Lake Pica, sediment PYR100

Fig. 5

Lake Pica Palòmera, sediment PYR52

Figs. 1-6 Manfred Ruppel photos 

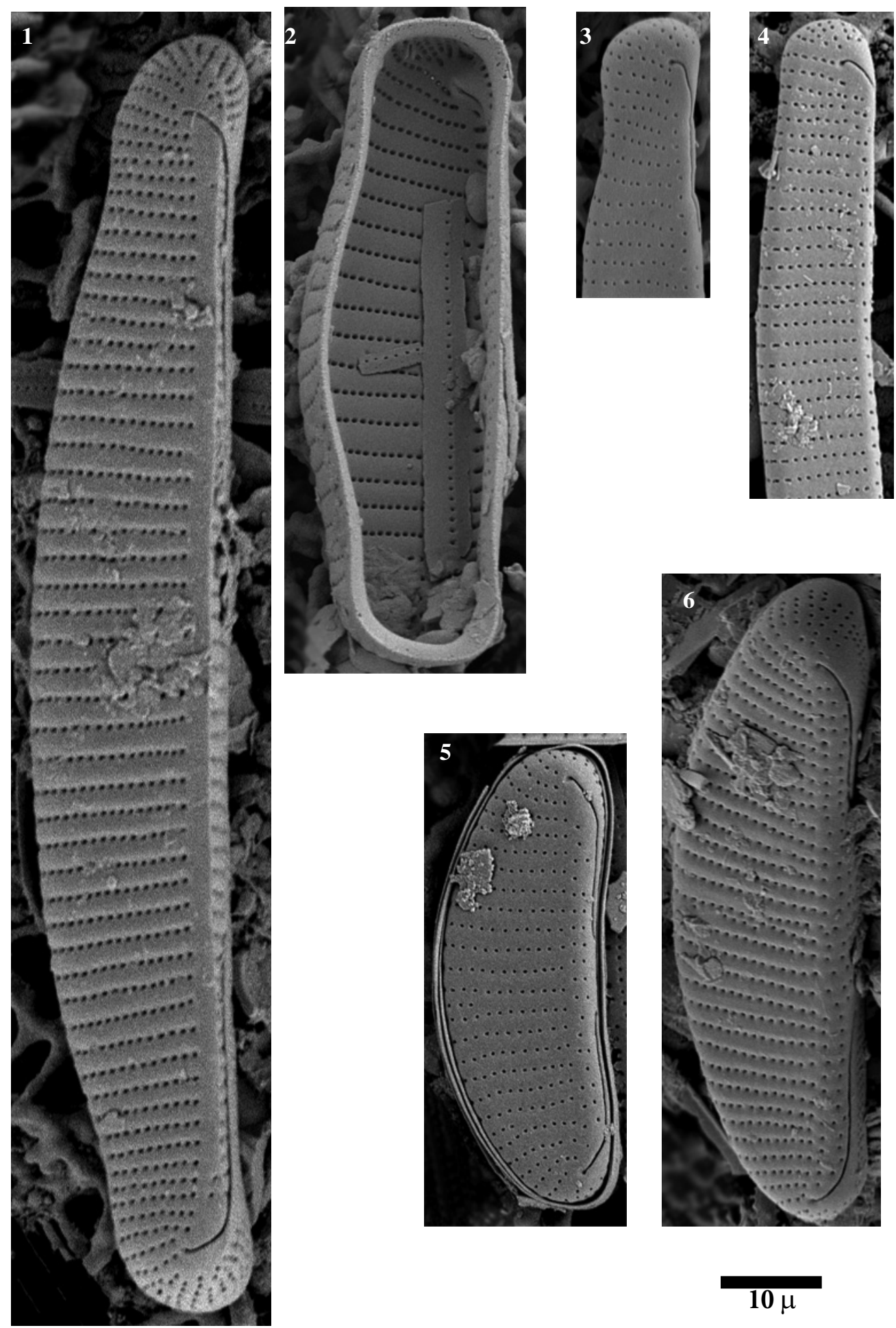
Plate 33

LM: x1500

Fig. $1 \quad$ Eunotia ambivalens Lange-Bertalot \& Tagliaventi

Figs. 2-7 Eunotia bilunaris (Ehrenberg) Schaarschmidt

Figs. 8-13 Eunotia mucophila (Lange-Bertalot \& Nörpel-Schempp) LangeBertalot

Fig. 14 Eunotia naegelii Migula

Figs. 15-19 Eunotia neocompacta var. vixcompacta Lange-Bertalot

Figs. 1, 4 Lake Sen, sediment PYR40

Fig. 2 Lake Inferior de la Gallina, sediment PYR87

Fig. $3 \quad$ Lake Forcat inferior, sediment PYR77

Figs. 5, 6, $7 \quad$ Lake Posets, sediment PYR42

Figs. 8-10, Lake Senó, epilithic EpiPYR84

12-13

Figs. 11, 15, Lake Monges, sediment PYR57

17,19

Fig. 14 Lake Mariola, sediment PYR80

Fig. 16 Lake Baiau superior, sediment PYR76

Fig. 18 Lake Senó, sediment PYR84 

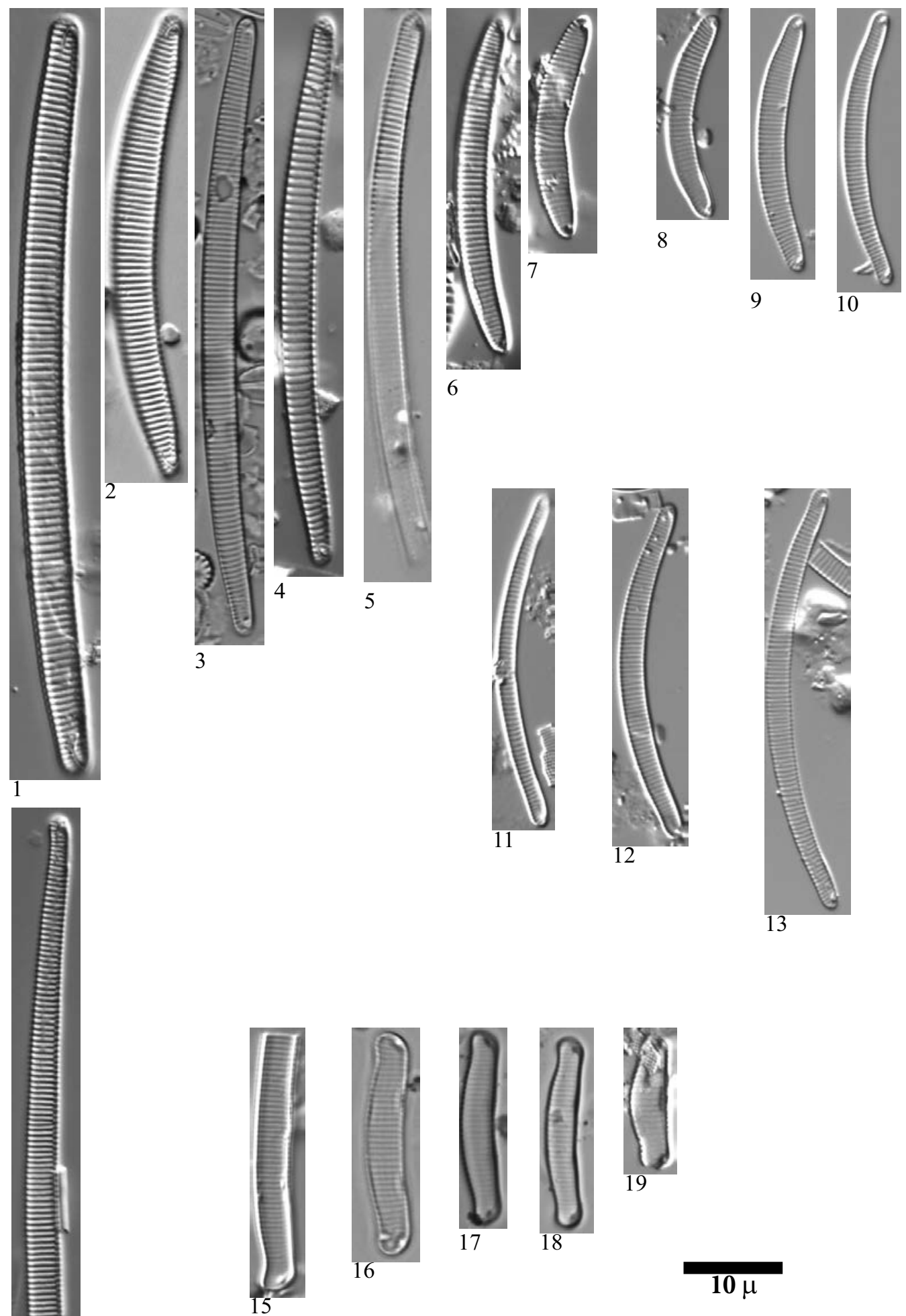

Limnetica, 36 (1): 127-395 (2017) 
SEM: x6000

Figs. 1-19, 25

Fig. 20

Fig. 21

Figs. 22-23, 26

Fig. 24

Fig. 27
Eunotia nymanniana Grunow

Eunotia cf. nymanniana Grunow

Eunotia cf. exigua (Brébisson Kutzing) Rabenhorst

Eunotia exigua (Brébisson Kutzing) Rabenhorst

Eunotia tenella (Grunow) Hustedt

Eunotia cf. exigua (Brébisson Kutzing) Rabenhorst

Figs. 1, 3-6, 8-20, 22, Lake Pica Palòmera, epilithic EpiPYR52 25-27

Fig. 2

Lake Aixeus, sediment PYR92

Fig. 7

Lake Negre, sediment PYR79

Fig. 21

Lake Baiau superior, sediment PYR76

Fig. 23

Lake Eriste, sediment PYR43

Fig. 24

Lake Estelat, sediment PYR120

Figs. 25-27

Manfred Ruppel photos 

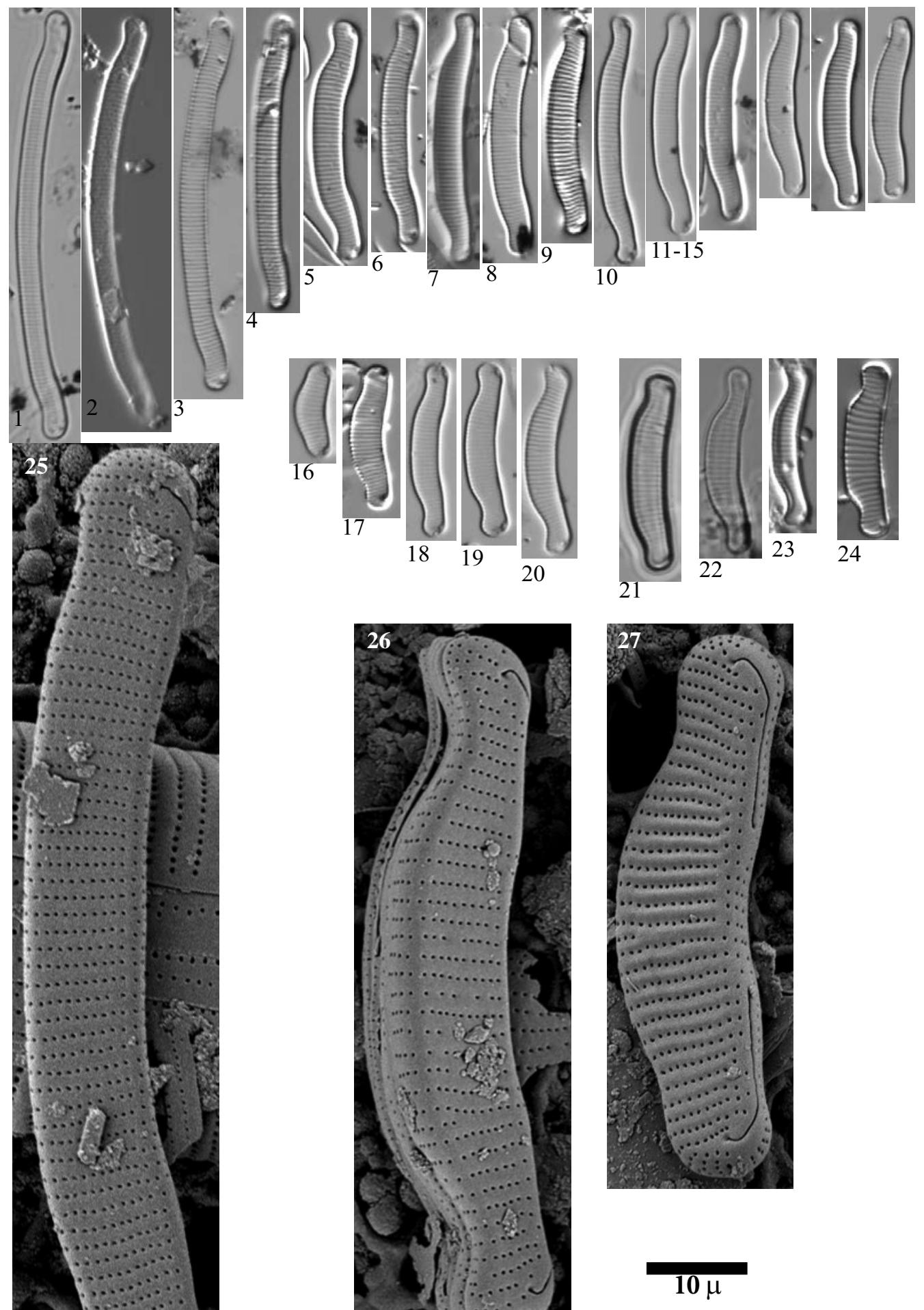
Plate 35 LM: $x 1500$

SEM: Fig. 1 x 6000, Fig. 2x2000

Figs. 1-3 Eunotia paludosa Grunow

Figs. 4-5 Eunotia neofallax Nörpel-Schempp \& Lange-Bertalot

Fig. 6 Eunotia groenlandica (Grunow) Norpel-Schempp \& Lange-Bertalot

Fig. 7 Eunotia cf. groenlandica (Grunow) Norpel-Schempp \& Lange-Bertalot

Fig. 8-10 Eunotia fallax A. Cleve

Fig. 11 Eunotia fallacoides Lange-Bertalot \& Cantonati

Fig. 12 Eunotia microcephala Krasske

Figs. 1-2 Lake Senó, sediment PYR84

Fig. 3 Lake Aubé, sediment PYR82

Figs. 4-5 Lake Cregüeña, sediment PYR49

Figs. 6, 10 Lake Aixeus, sediment PYR92

Fig. 7 Lake Pica Palòmera, sediment PYR52

Fig. 8 Lake Baiau superior, sediment PYR76

Fig. 9 Lake Romedo de Dalt, epilithic EpiPYR85

Fig. 11 Lake Illa, sediment PYR66

Fig. 12 Lake Monges, sediment PYR57 

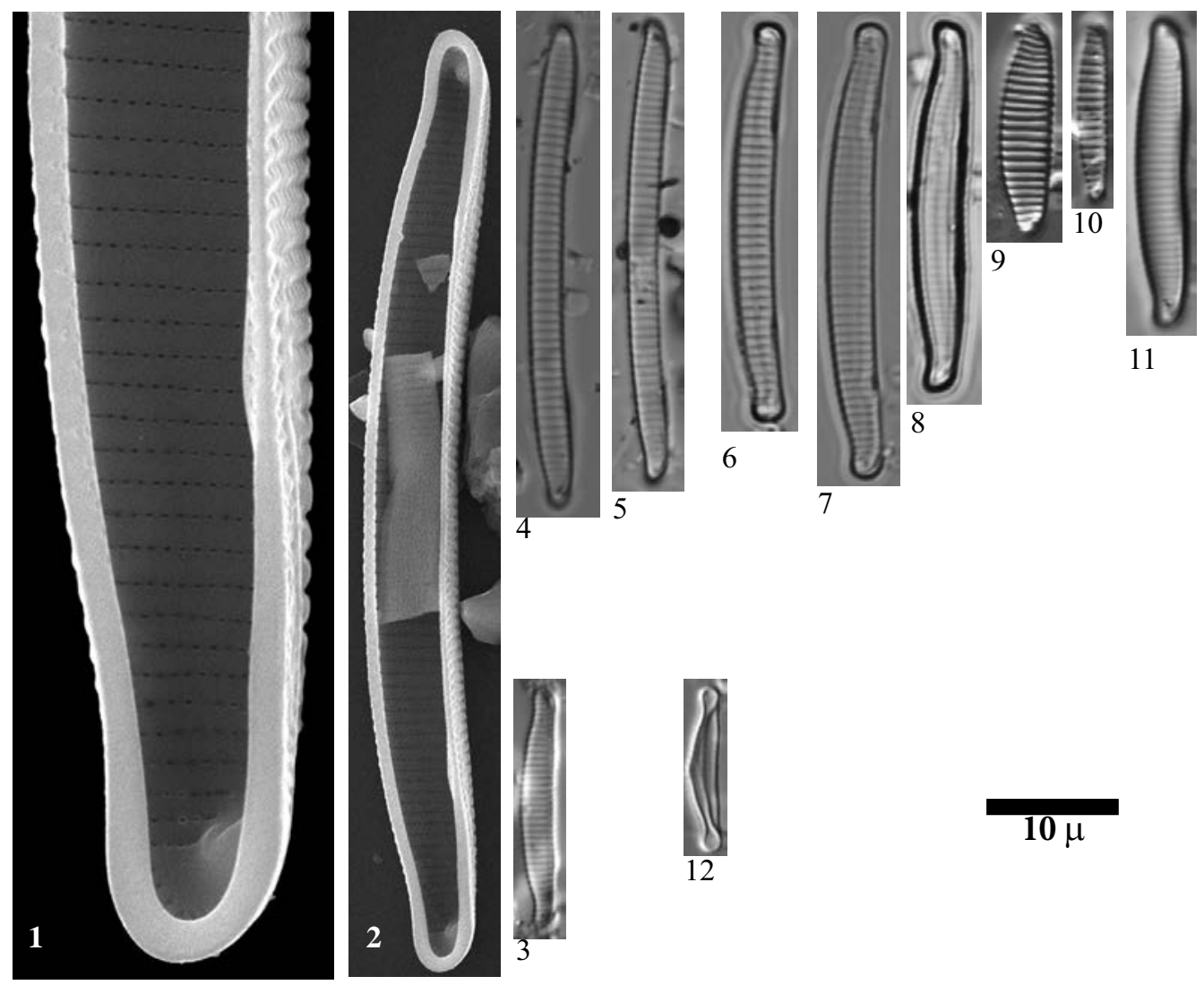
LM: x1500

SEM: Figs. 11,25,26 x 5000, Fig. 24 x8000

Figs. 1-2

Figs. 3-4

Figs. 5-6

Figs. 7-11

Fig. 12

Fig. 13

Figs. 14-15

Figs. 16-17

Fig. 18

Figs. 19-26
Eucocconeis coarctata (Brébisson) Lange-Bertalot

Eucocconeis flexella (Kützing) Meister

Eucocconeis alpestris (Brun) Lange-Bertalot

Eucocconeis laevis (Østrup) Lange-Bertalot

Psammothidium altaicum (Poretzky) Bukhtiyarova

Karayevia carissima (Lange-Bertalot) Bukhtiyarova

Karayevia oblongella (Østrup) Aboal

Karayevia laterostrata (Hustedt) Bukhtiyarova

Achnanthes cf. punctulata Simonsen

Karayevia suchlandtii (Hustedt) Bukhtiyarova

Figs. 1-2, 13, 18-23, Lake Posets, sediment PYR42

23-26

Figs. 3-4

Figs. 5-9

Fig. 10

Fig. 11

Fig. 12

Figs. 14-15

Figs. 16-17
Lake Ormiélas, sediment PYR05

Lake Llebreta, sediment PYR58

Lake Acherito, sediment PYR01

Lake Roumassot, epilithic EpiPYR04

Lake Romedo de Dalt, sediment PYR85

Lake Forcat Inf., sediment PYR77

Lake Tourrat, sediment PYR23 

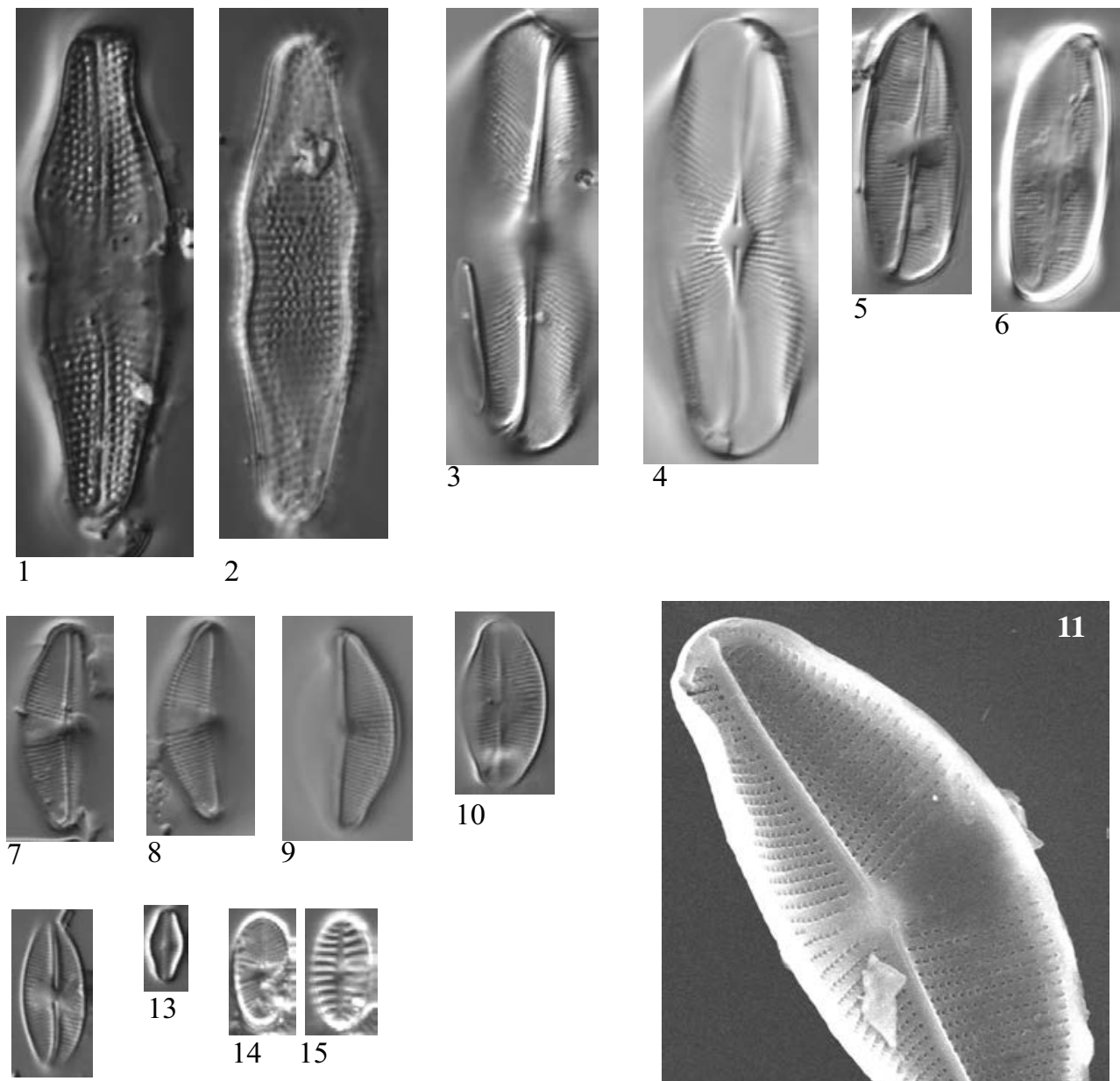

12

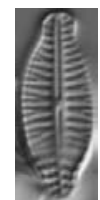

16
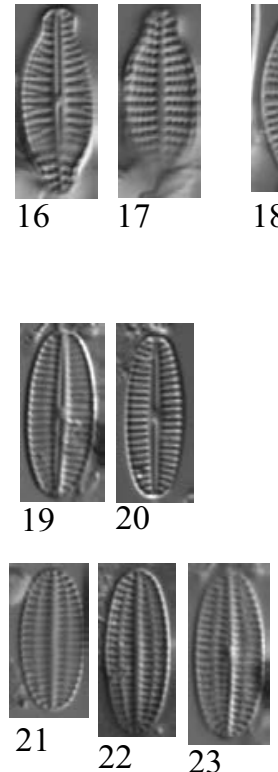
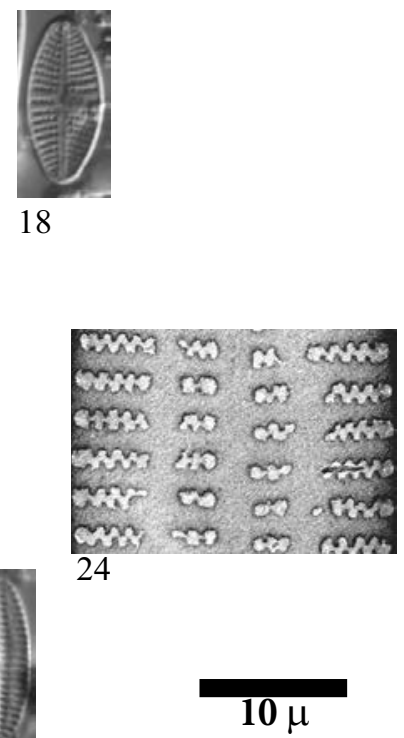
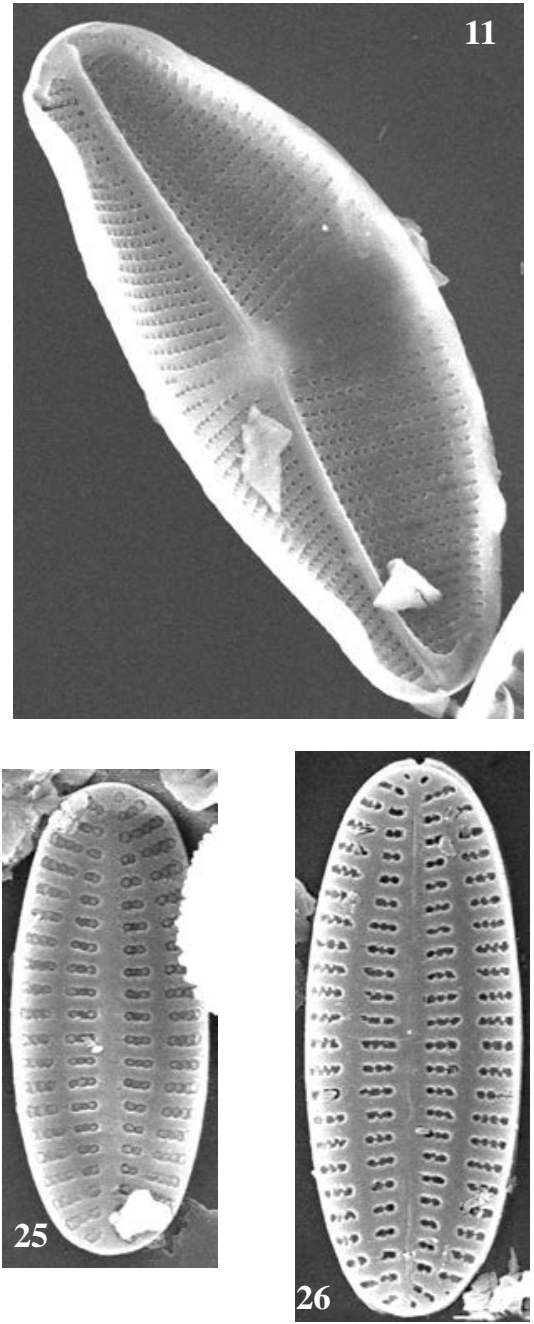
SEM: Figs. 12 x 4500, Fig. 14 x10000

Figs. 1-4

Figs. 5-8

Figs. 9-12

Fig. 13-14
Nupela cf. impexiformis (Lange-Bertalot) Lange-Bertalot

Nupela cf. gracillima (Hustedt) Lange-Bertalot

Nupela lapidosa (Krasske) Lange-Bertalot

Nupela silvahercynia (Lange-Bertalot) Lange-Bertalot

Figs. 1-2 Lake Compte, sediment PYR97

Figs. 3-4, 10, 12 Lake Garbet, sediment PYR81

Figs. 5-7

Lake Helado de Marboré, sediment PYR18

Fig. 8

Lake Long de Liat, sediment PYR55

Fig. 9

Lake Coronas, epilithic EpiPYR47

Fig. 11

Lake Posets, epilithic EpiPYR42

Fig. 13

Lake Cregüeña, epilithic EpiPYR49

Fig. 14 

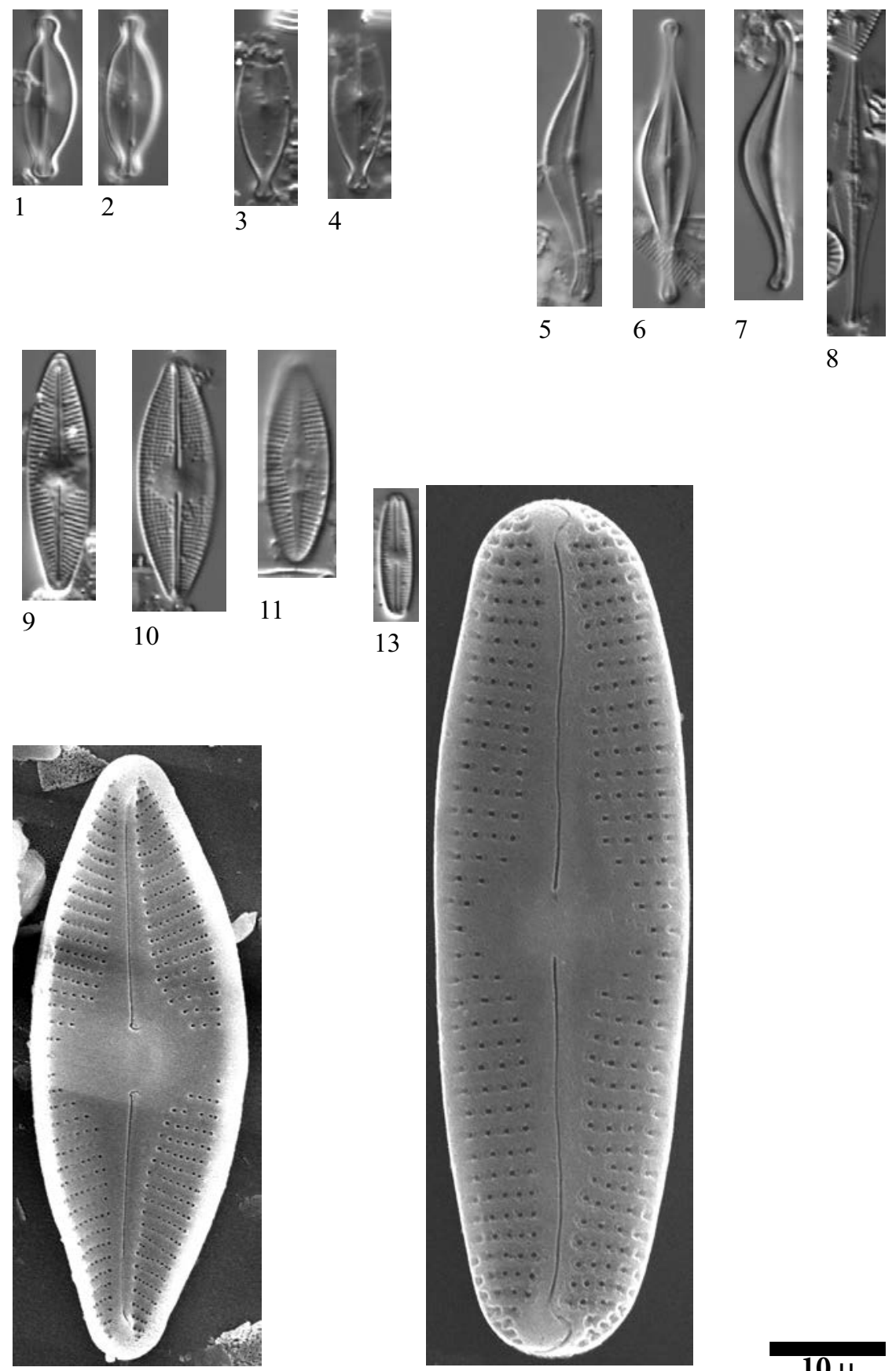

12

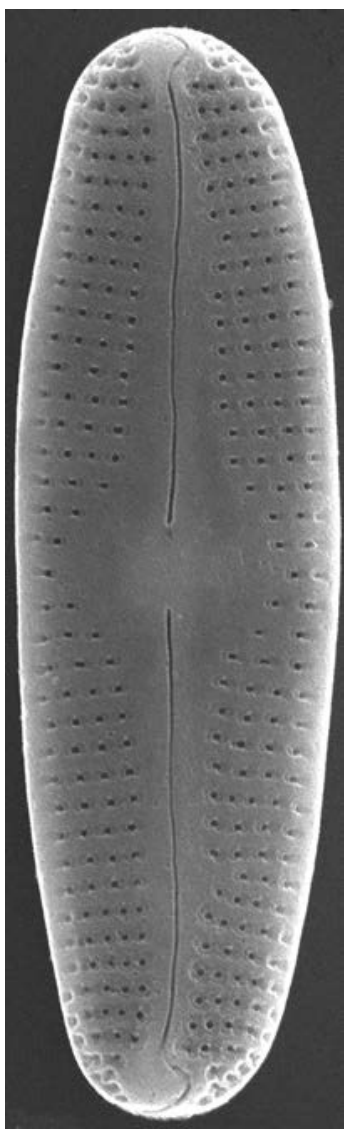

14 
Plate $38 \quad$ LM: $x 1500$

SEM: Fig. 7 x 6000, Fig. 12 x 4500, Fig. 22 x8000

Figs. 1-2 Planothidium cf. stewartii (Patrick) Lange-Bertalot

Figs. 3-7 Planothidium distinctum (Messikommer) Lange-Bertalot

Figs. 8-9 Planothidium cf. distinctum (Messikommer) Lange-Bertalot

Figs. 10-12 Platessa cf. conspicua (Mayer) Lange-Bertalot

Figs. 13-14 Planothidium lanceolatum (Brébisson ex Kützing) Lange-Bertalot

Figs. 15-18 Planothidium frequentissimum (Lange-Bertalot) Lange-Bertalot

Figs. 19-21 Planothidium sp. No. 1 Pondiellos

Fig. 22 Planothidium frequentissimum (Lange-Bertalot) Lange-Bertalot

Figs. 23-31 Planothidium rostratum (Østrup) Lange-Bertalot

Figs. 32-35 Planothidium oestrupii (Cleve-Euler) Edlund

Figs. 36-37 Planothidium peragalli (Brun \& Héribaud) Round et Bukhtiyarova

Figs. 38-41 Planothidium calcar (Cleve) M.B. Edlund

Figs. 1-2 Lake Trebens, sediment PYR114

Figs. 3-9, Lake Posets, sediment PYR42

34-35

Figs. 10-12, Lake Laurenti, sediment PYR111

22

Figs. 13-14 Lake La Munia, sediment PYR20

Figs. 15-16, Lake Estom, sediment PYR15 40-41

Figs. 17-18 Lake Arratille, sediment PYR11

Figs. 19-20 Lake Pondiellos, sediment PYR08

Fig. $21 \quad$ Lake Cap Long, sediment PYR24

Figs. 23-31 Lake Burg

Figs. 32-33 Lake Sen, sediment PYR40

Figs. 36-37 Lake Sen, sediment PYR120

Fig. 38 Lake Barsau, sediment PYR03

Fig. 39 Lake Acherito, sediment PYR01 

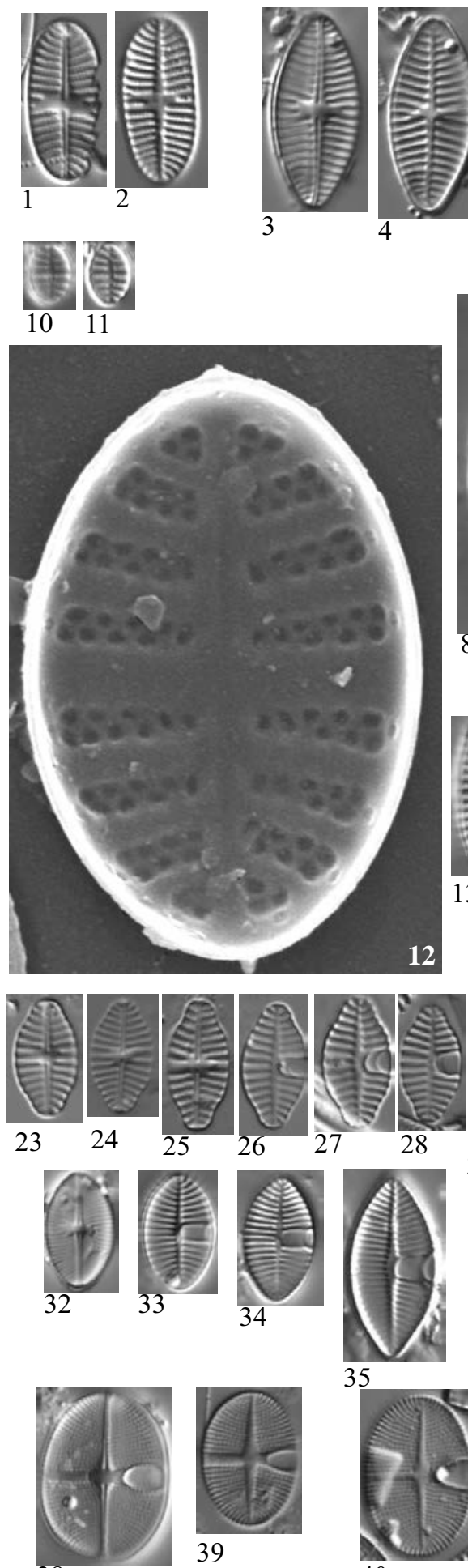

38
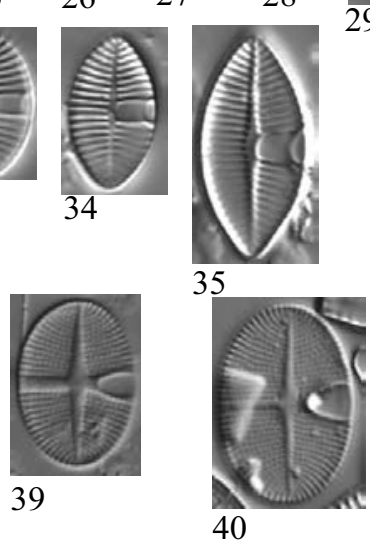
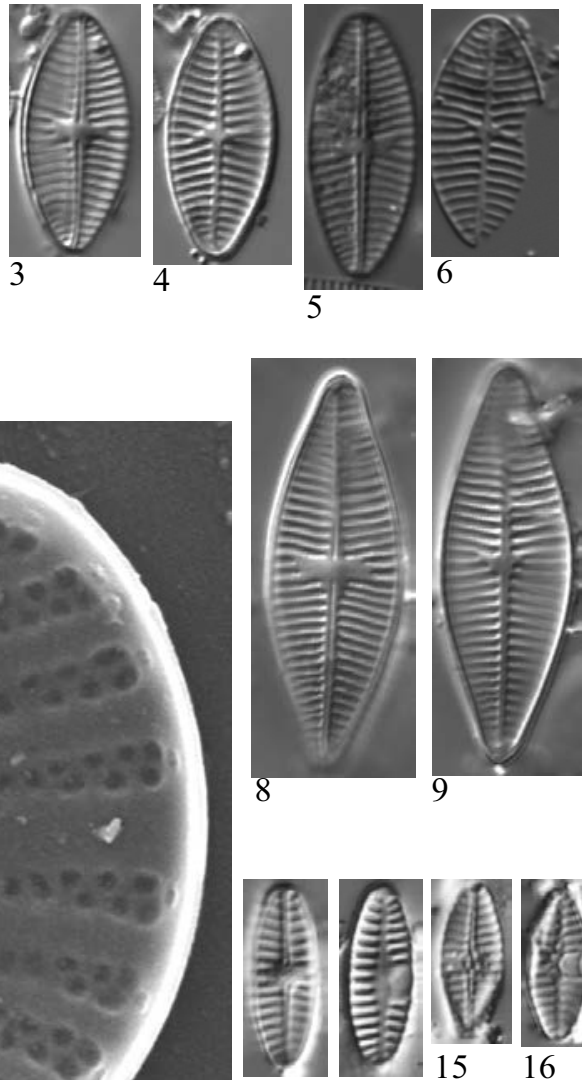

13

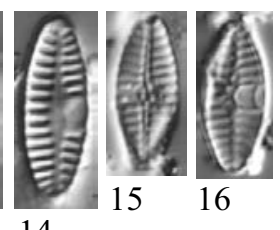

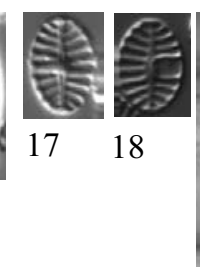
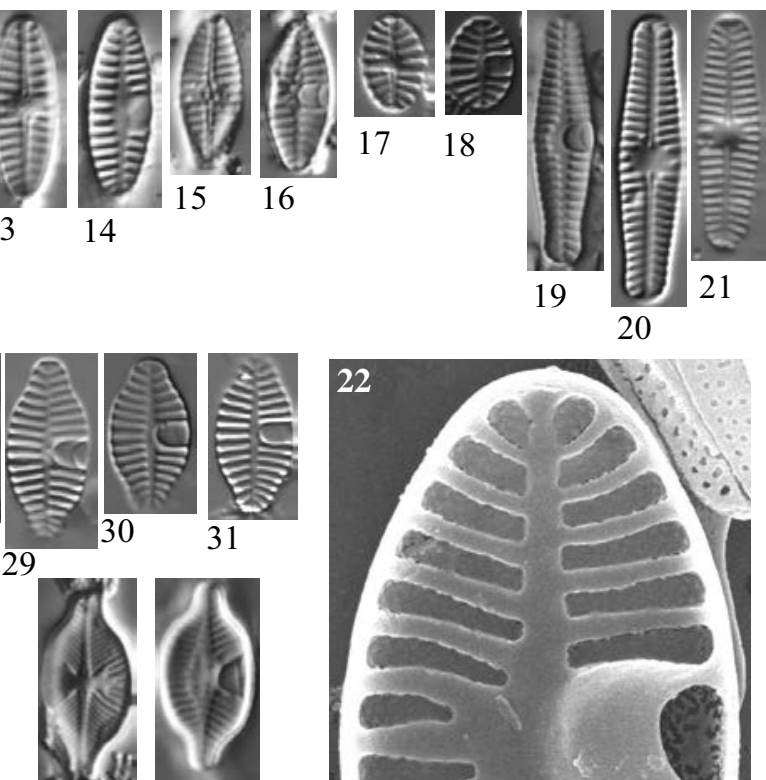

36
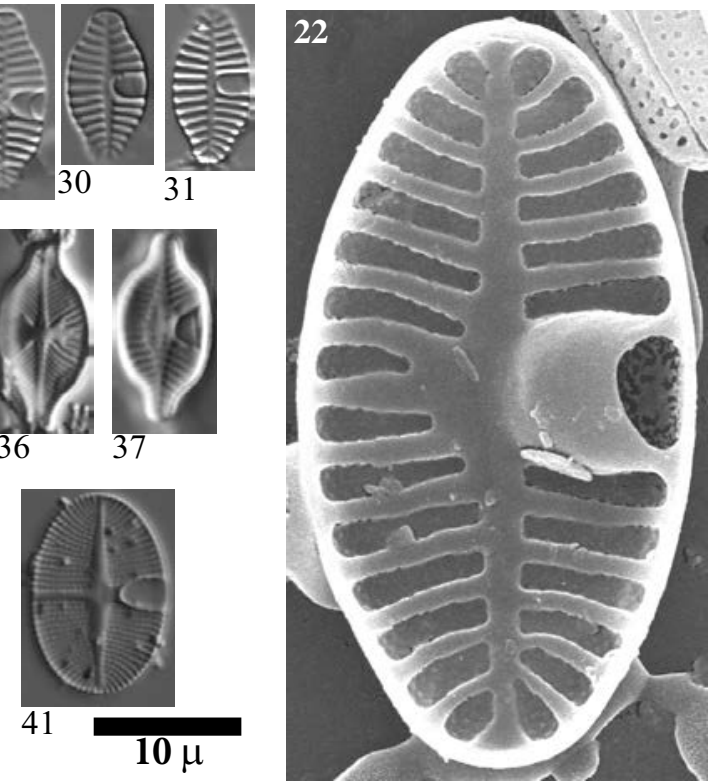


$\begin{array}{ll}\text { Plate } 39 & \text { LM: } x 1500 \\ & \text { SEM: } x 10000\end{array}$

Figs. 1-13, Psammothidium microscopicum (Cholnoky) S.Blanco $18-21$

Figs. 14-17, Psammothidium cf. microscopicum (Cholnoky) S.Blanco 28-31

Figs. 22-27 Achnanthes sp. No. 2 Burg

Figs. 1-17, 19, Lake Posets, sediment PYR42 20-30

Figs. 18, 20, 21， Lake Redon, sediment REDOM 28

Figs. 23-27 Lake Burg, sediment BURG 953

Fig. 31 Lake Angonella, epilithic EpiPYR78 

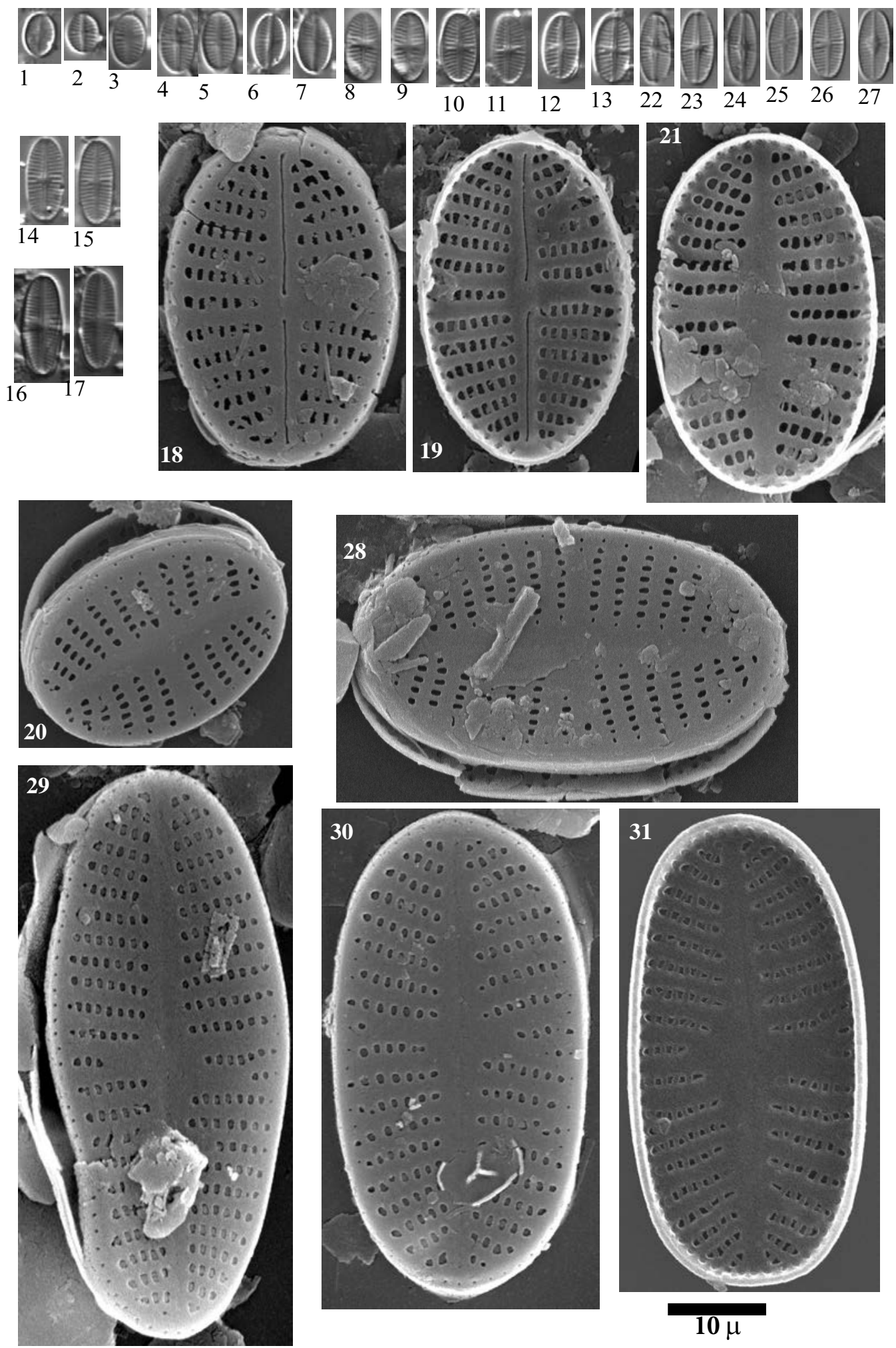
LM: $x 1500$

SEM: $x 10000$

Figs. 1-6

Figs. 7-15

Figs. 16-26

Fig. 27
Psammothidium didymum (Hustedt) Bukhtiyarova \& Round

Psammothidium subatomoides (Hustedt) Bukhtiyarova et Round

Psammothidium levanderi (Hustedt) Bukhtiyarova \& Round Psammothidium cf. levanderi (Hustedt) Bukhtiyarova \& Round

Figs. 1-2, 5-6

Lake Les Laquettes, sediment PYR27

Figs. 3-4, 7-12, 16-21

Lake Posets, sediment PYR42

25-26

Figs. 14-15

Lake Mariola, sediment PYR80

Figs. 22-24

Lake Sen, sediment PYR40

Fig. 27 

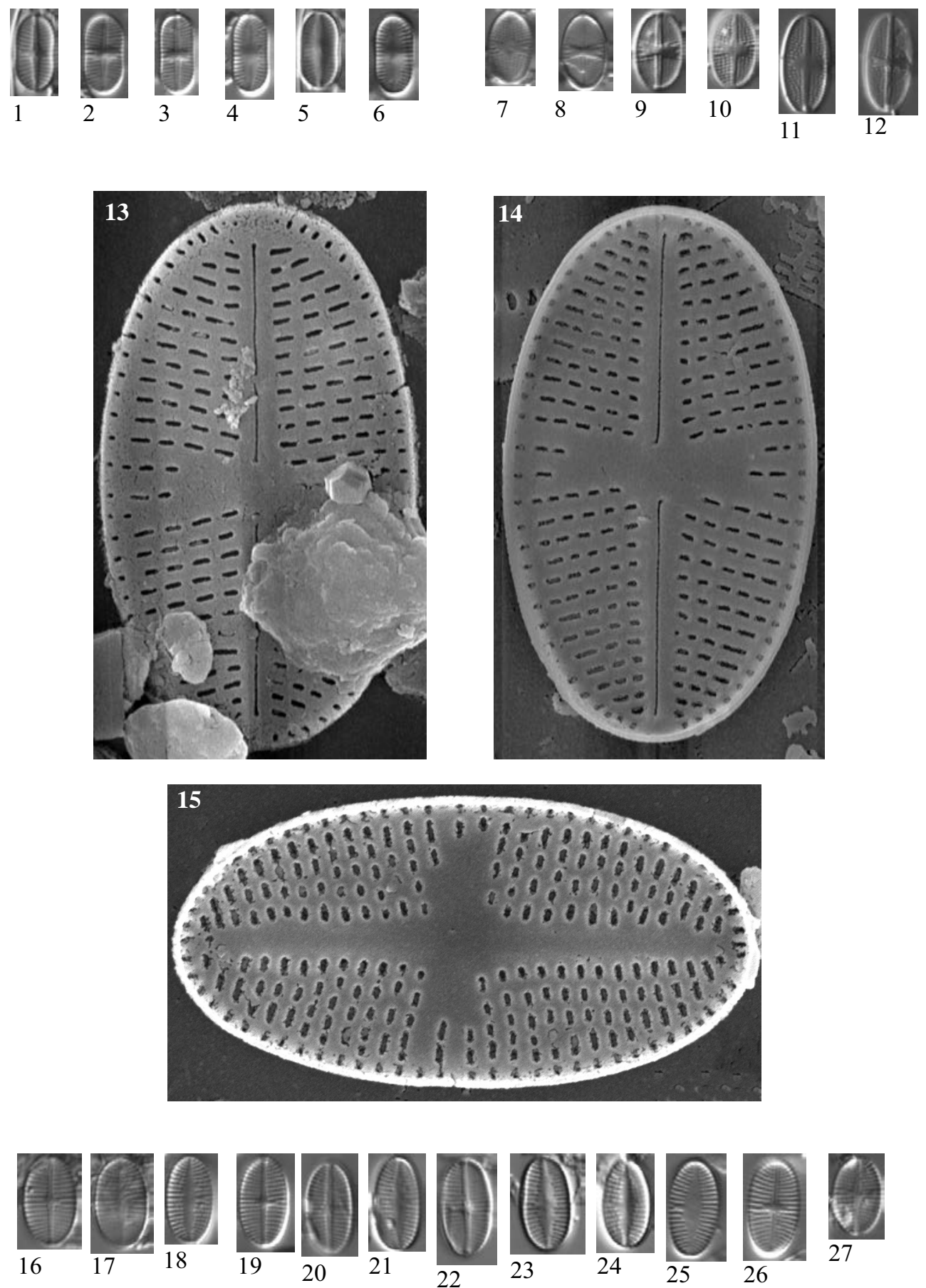

27

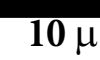


LM: x1500

SEM: Fig. 8 x10000 Figs. 18,19, 24 x9000

Figs. 1-8

Achnanthidium sp. No. 1 Posets

Figs. 9-19

Achnanthidium cf. minutissimum (Kützing) Czarnecki sensu lato

Figs. 20-21

Achnanthidium cf. kranzii (Lange-Bertalot) Round \& Bukhtiyarova

Figs. 22-24

Psammothidium rosenstockii (Lange-Bertalot) Lange-Bertalot

Fig. 25

Achnanthes sp. No. 6 Pessó

Figs. 26-32

cf. Achnanthidium atomus (Hustedt) Monnier, Lange-Bertalot \& Ector

Figs. 33-36 Achnanthes sp. No. 5 Posets

Figs. 1-7, 8, Lake Posets, sediment PYR42

33-35

Figs. 9-12 Lake Les Laquettes, sediment PYR27

Figs. 13-15, Lake Llebreta, sediment PYR58

26-27

Figs. 16-17 Lake Gerber, sediment PYR63

Figs. 18-19 Lake Laurenti, sediment PYR111

Figs. 20-21 Lake Eriste, sediment PYR43

Figs. 22-23 Lake Arratille, sediment PYR11

Fig. $24 \quad$ Lake Gran de Mainera, sediment PYR70

Fig. 25 Lake Gran del Pessó, sediment PYR56

Figs. 28-29 Lake La Munia Superior, sediment PYR20

Figs. 30-32 Lake Glacé, sediment PYR17

Fig. $36 \quad$ Lake Garbet, sediment PYR81 

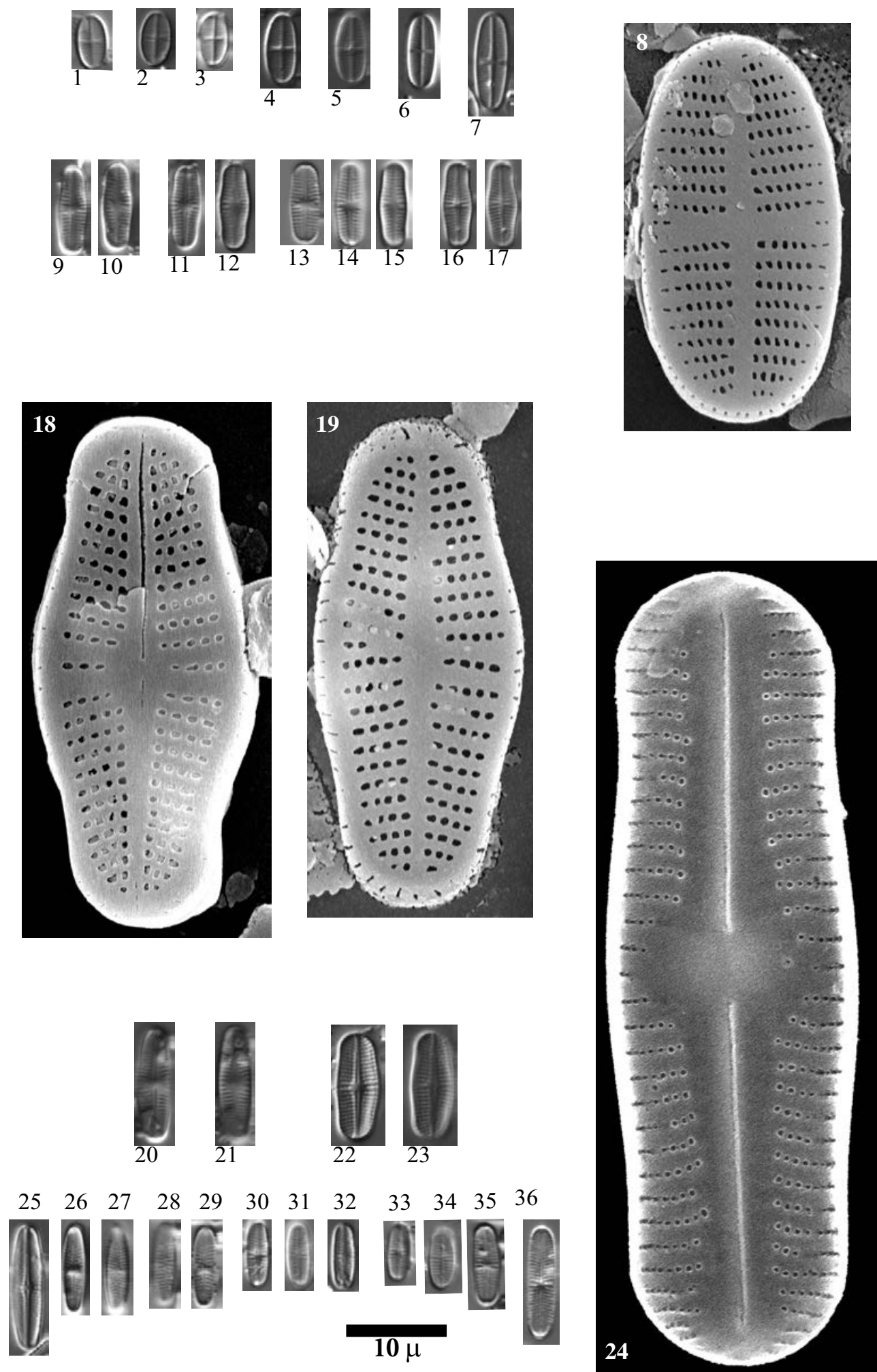
SEM: Figs. 3-8 x 9000 Fig. 9 x20000

Figs. 1-9 Achnanthidium minutissimum (Kützing) Czarnecki sensu lato

Figs. 10-20 Achnanthidium minutissimum (Kützing) Czarnecki sensu lato

Figs. 21-24 Achnanthidium cf. minutissimum (Kützing) Czarnecki

Fig. 25

Achnanthidium sp

Figs. 1-2 Lake Llebreta, sediment PYR58

Figs. 3-5, 9 Lake Roumassot, sediment PYR04

Figs. 6-8 Lake Roumassot, epilithic EpiPYR04

Figs. 10.12 Lake Chelau Sup., sediment PYR41

Fig. $13 \quad$ Lake Burg

Figs. 14-24 Lake Les Laquettes, sediment PYR27

Fig. 25 Lake Pixón, sediment PYR44 

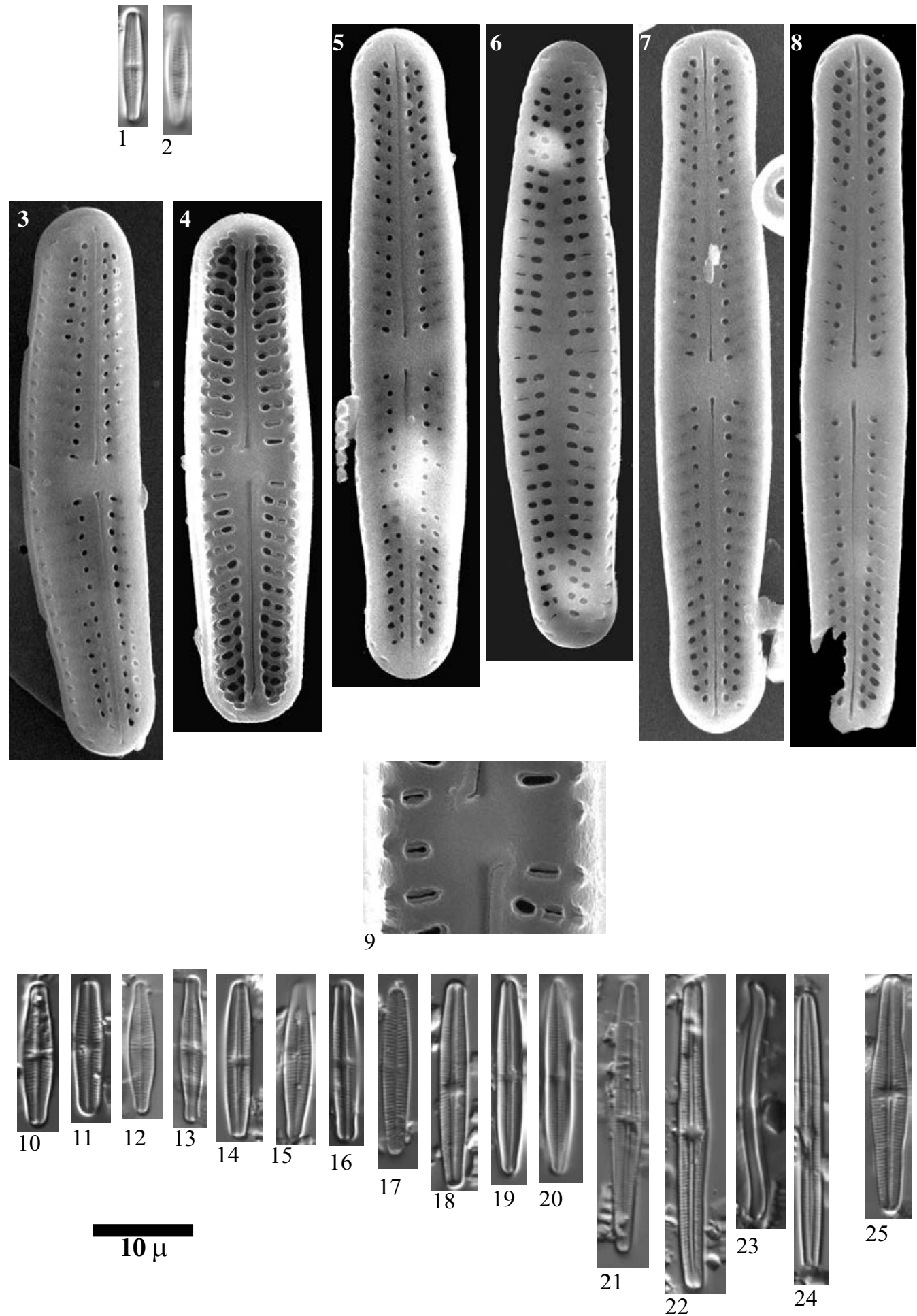
Figs. 1-2, 11-20

Figs. 3, 8

Figs. 4, 6

Figs. 5, 7

Fig. 9

Fig. 10

Figs. 21-22

Fig. 23

Fig. 24

Figs- 25-27
Lake Posets, sediment PYR42

Lake Port Bielh, epilithic EpiPYR28

Lake Roumassot, epilithic EpiPYR04

Lake Pondiellos, epilithic EpiPYR08

Lake Acherito, epilithic EpiPYR01

Lake Mariola, epilithic EpiPYR80

Lake Aubé, sediment PYR82

Lake Burg, sediment BURG 932

Lake Burg, sediment BURG 1007

Lake Burg, sediment BURG 831 

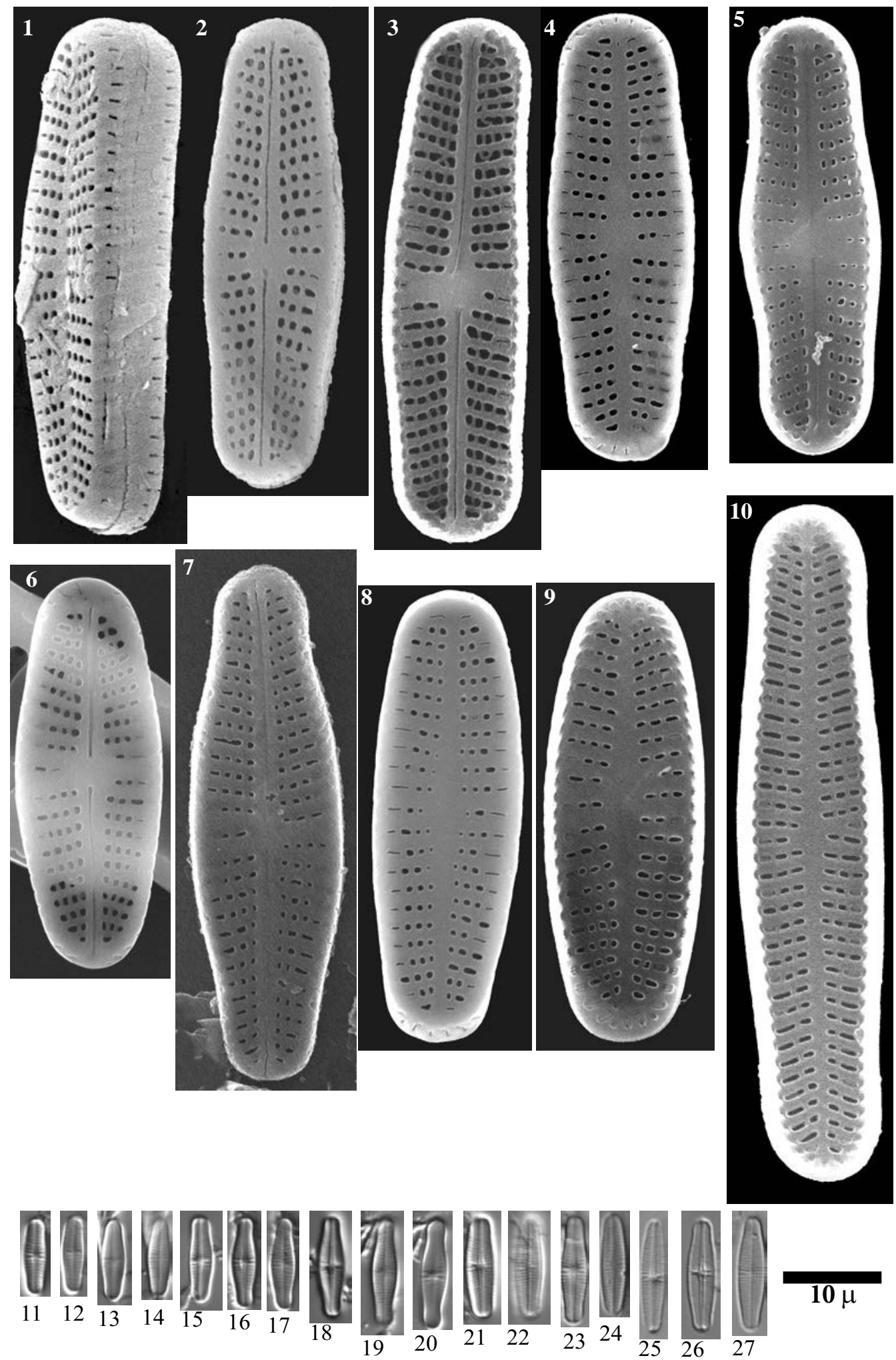
Figs. 1-16

Figs. 17-24

Figs. 1-2, 5-9

Figs. 3-4, 23-24

Figs. 10, 12

Fig. 13

Figs. 14-15, 19-22

Figs. 16, 18

Figs. 17
Achnanthidium minutissimum (Kützing) Czarnecki sensu lato

cf. Achnanthidium catenatum (Bily \& Marvan) H. LangeBertalot

Lake Llebreta, sediment PYR58

Lake Bersau, sediment PYR03

Lake Estom, sediment PYR15

Lake Arratille, epilithic EpiPYR11

Lake Posets, sediment PYR42

Lake Port Bielh, epilithic EpiPYR28

Lake Laurenti, sediment PYR111 

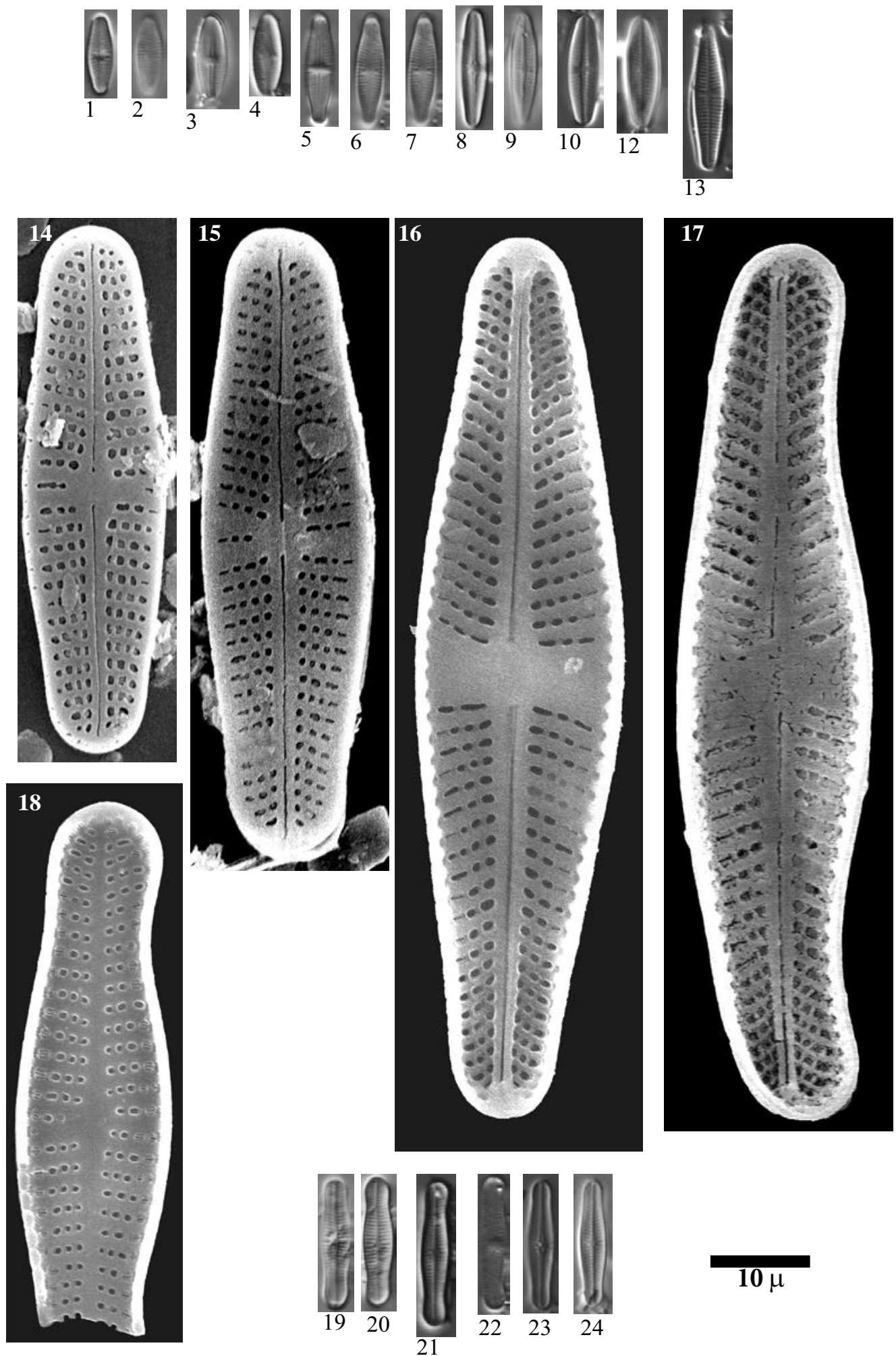

$10 \mu$ 
Plate $45 \quad$ LM: $x 1500$

SEM: $\mathrm{x} 9000$

Figs. 1-5 Achnanthidium minutissimum (Kützing) Czarnecki

Fig. 1 Lake, sediment BURG 939

Figs. 2-5 Lake Redon, sediment REDOM 

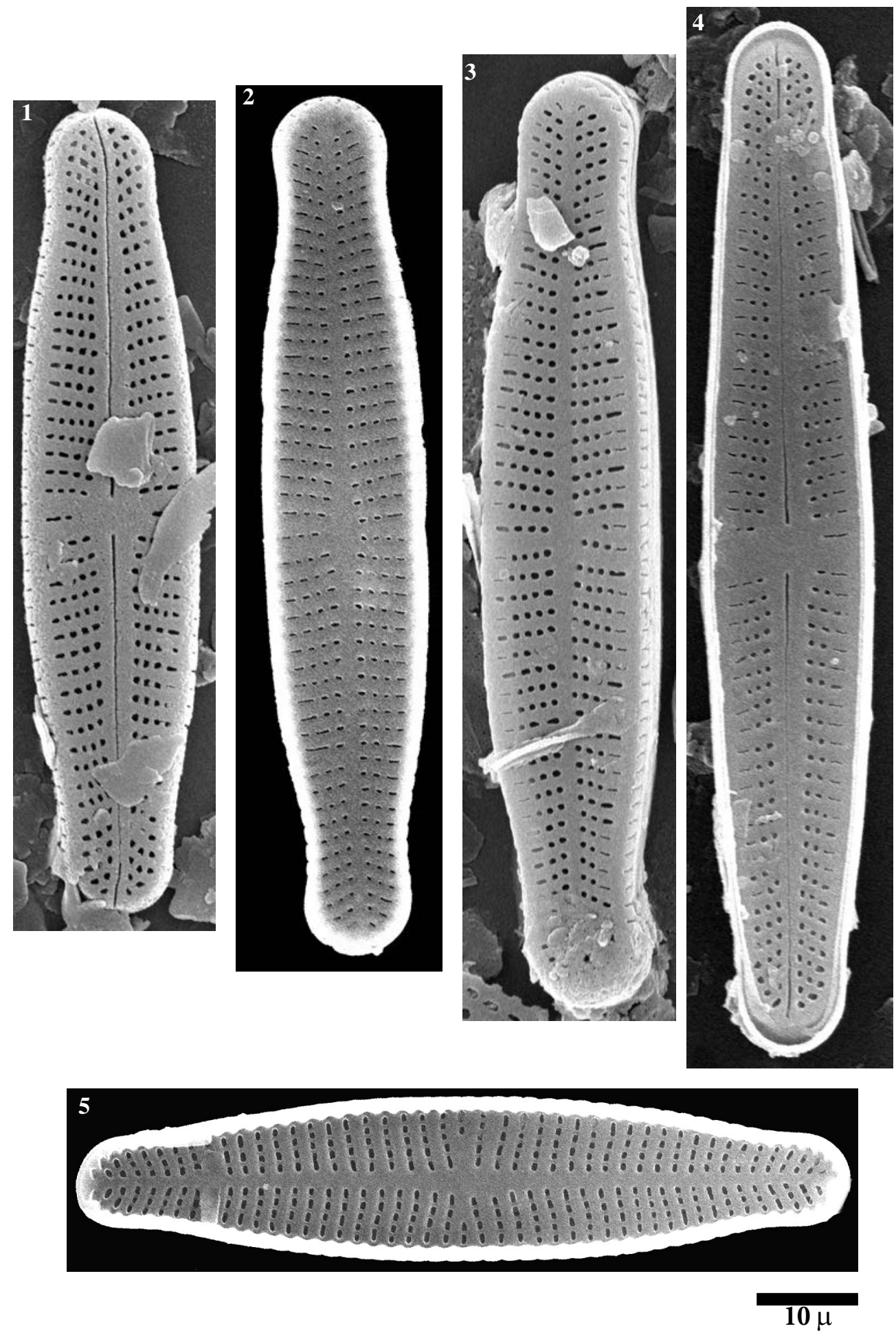
SEM: $1-4$ x9000, 5 x 15000

Figs. 1-3, 5-10 Achnanthidium minutissimum (Kützing) Czarnecki

Fig. 4 Achnanthidium caledonicum (Lange-Bertalot) Lange-Bertalot

Fig. 1 Lake Roumassot, sediment PYR04

Figs. 2, 4-5 Lake Port Bielh, epilithic EpiPYR28

Fig. $3 \quad$ Lake Angonella, epilithic EpiPYR78

Figs. 6, 9-10 Lake Les Laquettes, sediment PYR27

Figs. 7-8 Lake Posets, sediment PYR42 

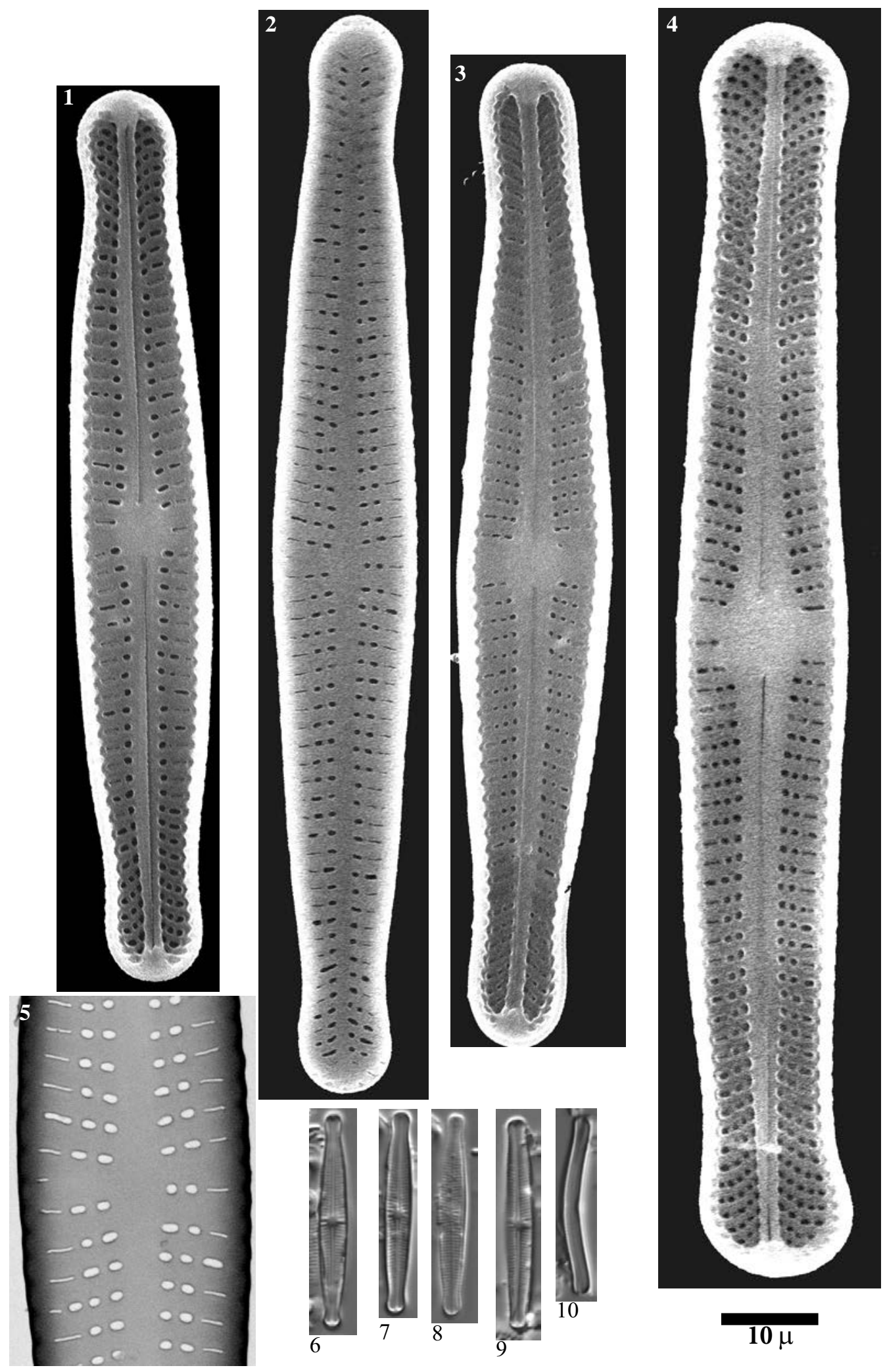

Limnetica, 36 (1): 127-395 (2017) 
LM: x1500

SEM: x9000

Figs. 1-3

Fig. 4-11, 21-24

Figs. 12-18

Fig. 25

Fig. 1

Figs. 2-3

Figs. 4-11, 21-24

Figs. 12-13

Figs. 14-16

Figs. 17-18

Figs. 19-20

Fig. 25
Achnanthidium minutissimum (Kützing) Czarnecki

Achnanthidium cf. minutissimum (Kützing) Czarnecki

Achnanthes sp. No. 8 Angonella

Achnanthidium sp.

Lake Les Laquettes, sediment PYR27

Lake Posets, epilithic EpiPYR42

Lake Burg

Lake Siscar, epilithic EpiPYR98

Lake Angonella, epilithic EpiPYR78

Lake Basa de la Mora, epilithic EpiPYR32

Lake Eriste, epilithc EpiPYR43

Lake Port Bielh, epilithic EpiPYR28 

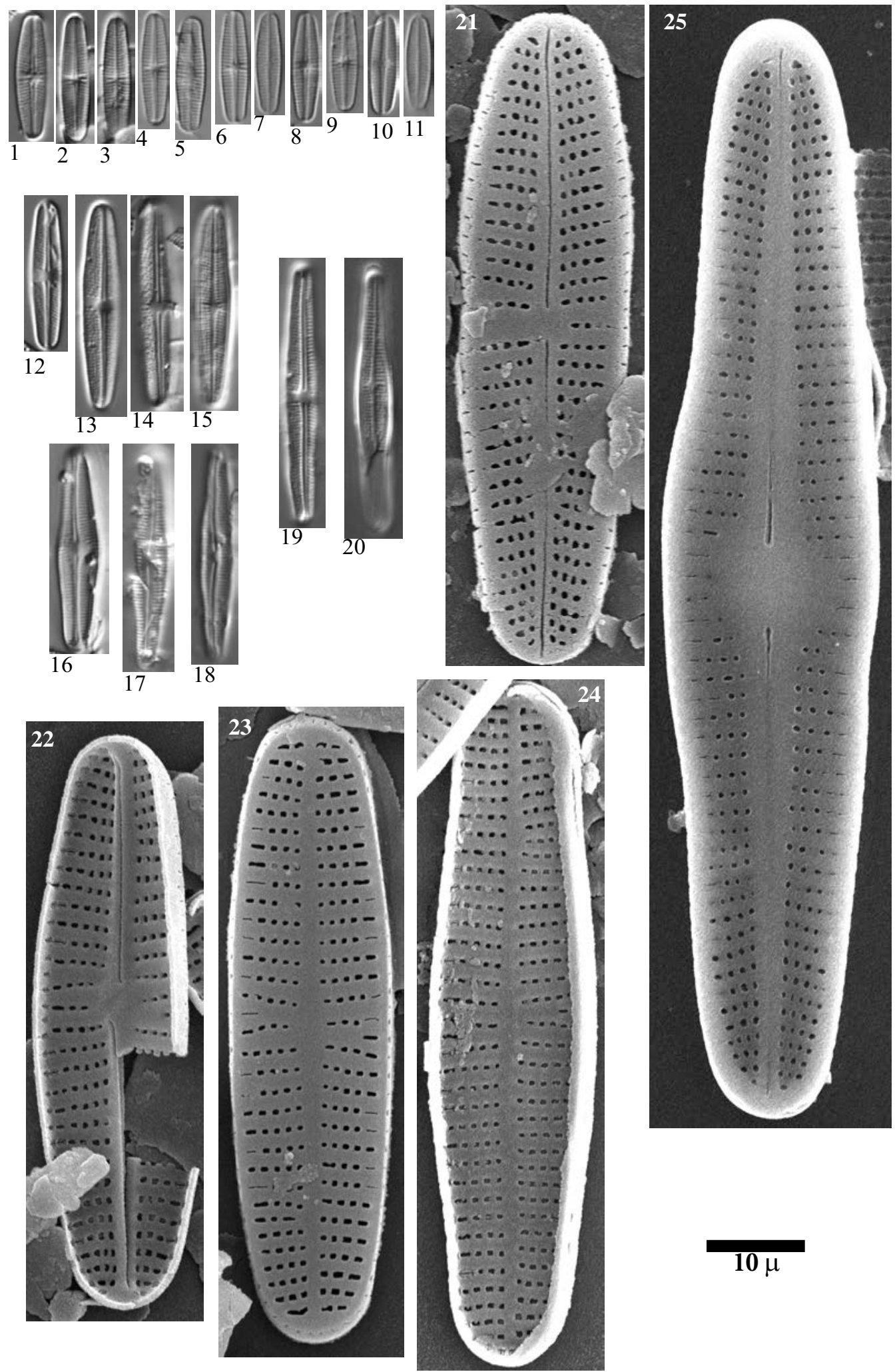

Limnetica, 36 (1): 127-395 (2017) 
Plate $48 \quad$ LM: $x 1500$

SEM: $x 10000$

Figs. 1-9

Figs. 10-11

Figs. 12-42

Figs. 43-46

Figs. 47-50

Fig 51

Figs. 1-9

Figs. 10-11

Figs. 12-13

Figs. 14-15, 43-45

Figs. 16, 17-30, $32-42,51$

Fig. 31

Fig. 36

Fig. 46

Fig. 56

Figs. 48-50
Achnanthidium pfisteri Lange-Bertalot

Achnanthidium cf. pfisteri Lange-Bertalot

Achnanthidium pyrenaicum (Hustedt) Kobayasi

Rossithidium pusillum (Grunow) Round et Bukhtiyarova

Rossithidium linearis (Smith) Round et Bukhtiyarova

Rossithidium petersenii (Hustedt) Round et Bukhtiyarova

Lake Helado de Marboré, sediment PYR18

Lake Filià, epilithic EpiPYR71

Lake Negre, sediment PYR11

Lake Les Laquettes, sediment PYR27

Lake Llebreta, sediment PYR58

Lake Estom, sediment PYR15

Lake Roumassot, epilithic EpiPYR04

Lake Posets, Sediment PYR42

Lake Coronas, sediment PYR47

Lake L'Estagnol, sediment PYR119 

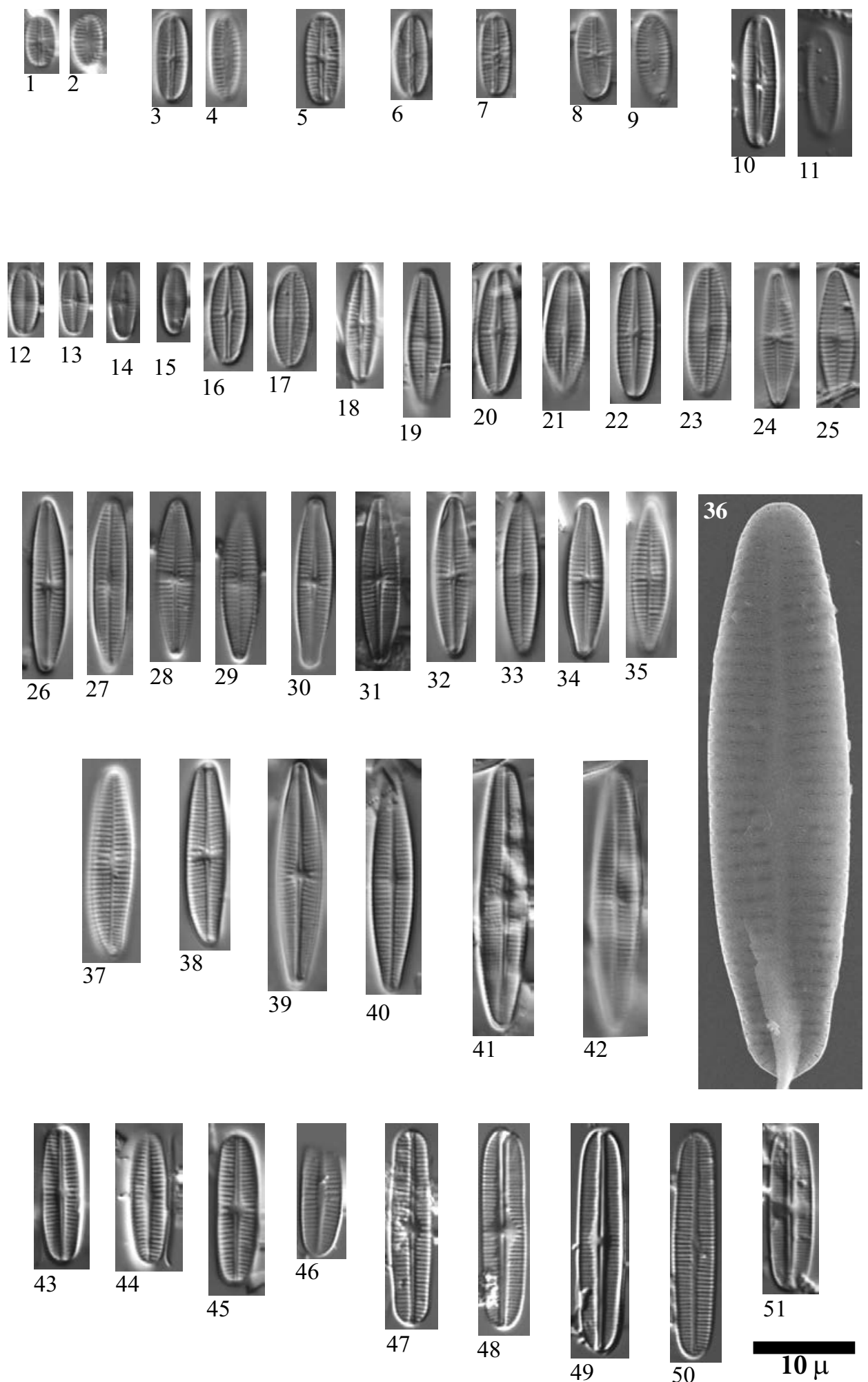
LM: x1500

SEM: Fig. 25 x11000, Fig. 26 x4000, Fig. 27-28 x 7000

Figs. 1-12

Psammothidium helveticum (Hustedt) Bukhtiyarova et Round

Figs. 13-27

Achnanthes helvetica var. minor Flower \& Jones

Figs. 28-29

Psammothidium helveticum (Hustedt) Bukhtiyarova et Round

Fig. 30

Psammothidium sp

Figs. 1-2

Figs. 3-5

Figs. 6-7

Figs. 8, 29

Figs. 9-10

Figs. 11-12

Figs. 13-22, 25-26

Figs. 23-24

Fig. 27

Fig. 28

Fig. 30
Lake Blaou, sediment PYR94

Lake Posets, sediment PYR42

Lake Llosás, sediment PYR46

Lake Mariola, sediment PYR80

Lake Forcat Inf., sediment PYR77

Lake Bleu de Rabassoles, sediment PYR112

Lake Negre, sediment PYR79

Lake Cregüeña, Sediment PYR49

Lake Garbet, sediment PYR81

Lake Redon, sediment REDOM

Lake Illa, sediment PYR66 

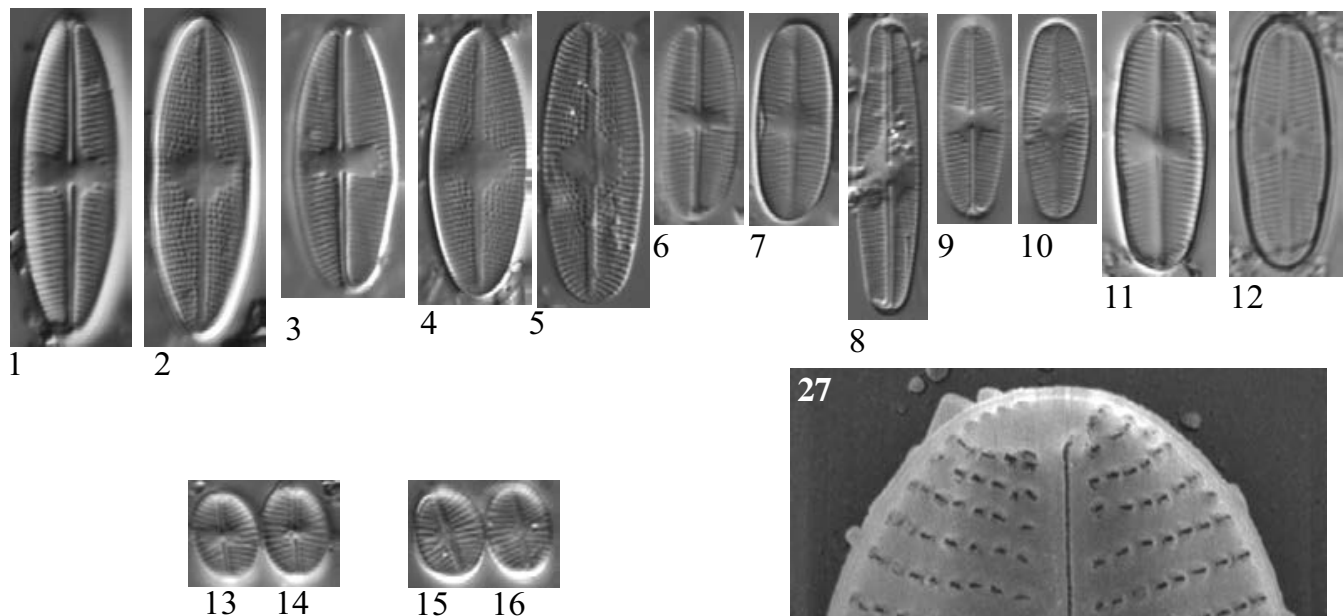

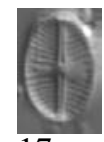

17
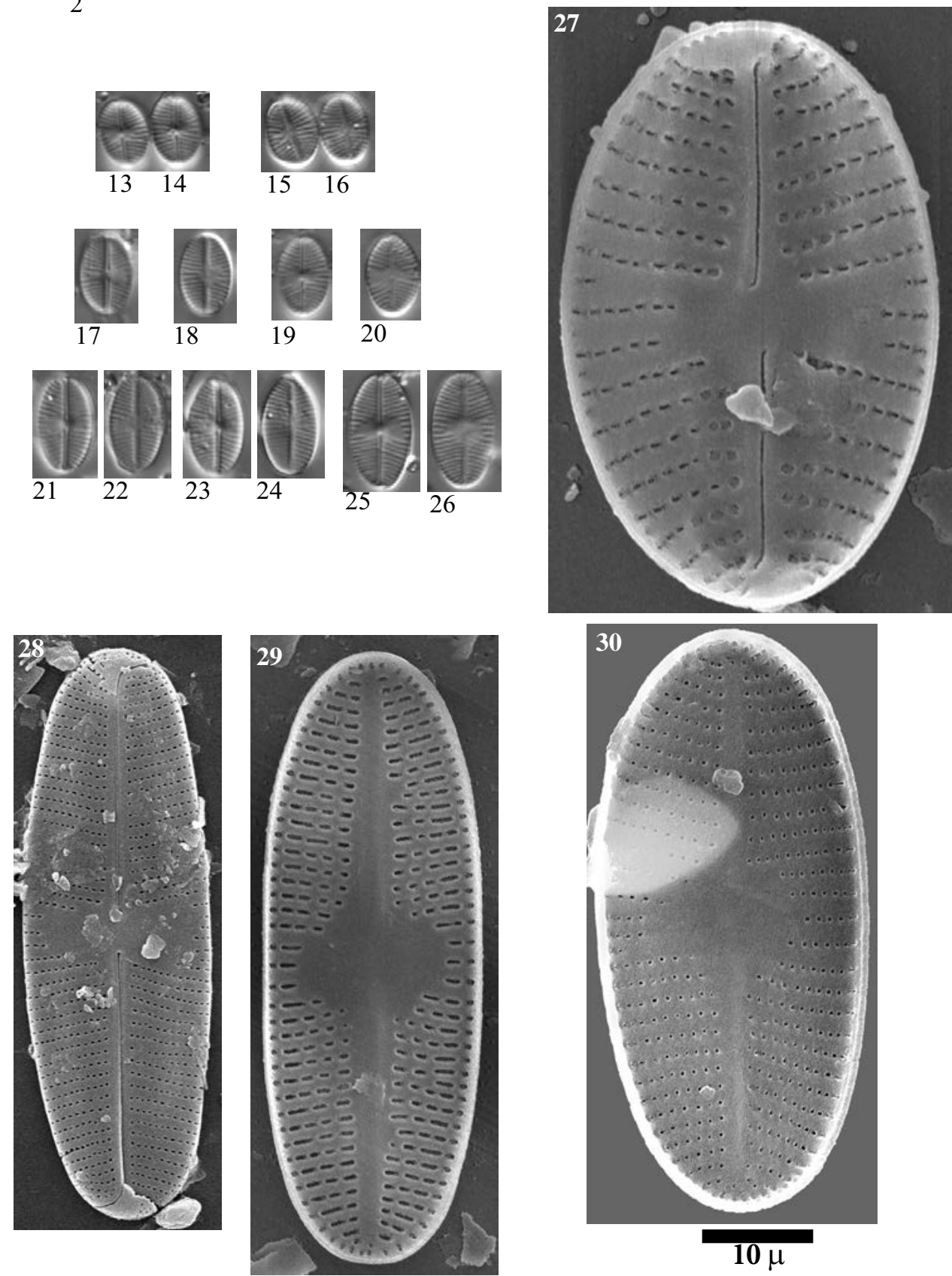

Limnetica, 36 (1): 127-395 (2017) 
Plate $50 \quad$ LM: $x 1500$

SEM: x 9000

Figs. 1-10 Psammothidium scoticum (Flower et Jones) Bukhtiyarova \& Round

Figs. 1-3 Lake Posets, sediment PYR42

Fig. $4 \quad$ Lake Monges, sediment PYR57

Fig. $5 \quad$ Lake Eriste, sediment PYR43

Figs. 6-10 Lake Redon, sediment REDOM

Fig. 11 Lake Garbet, sediment PYR81 

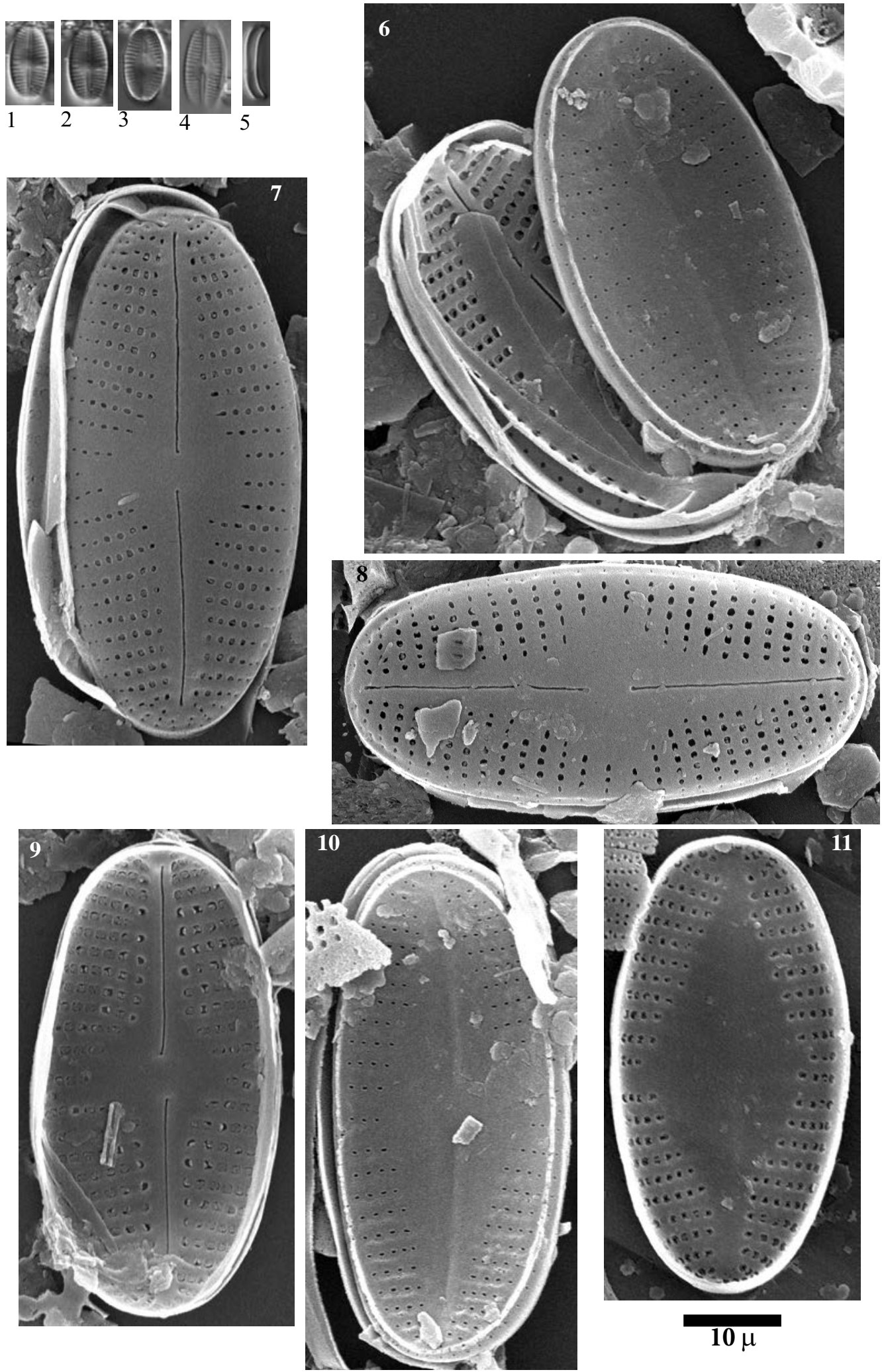

Limnetica, 36 (1): 127-395 (2017) 
Plate $51 \quad$ LM: $x 1500$

SEM: $\times 7000$

Figs. 1-3

Figs. 4-6

Figs. 7-9

Figs. 10-22

Figs. 23-24

Figs. 25-31

Figs. 32-36

Figs. 37-41

Figs. 42-43

Figs. 44-49

Figs. 50-53

Figs. 1-2, 23-24

Figs. 3, 6, 20-22

Figs. 4-5

Figs. 7-9, 44-49

Figs. 10-11, 32-

34

Figs. 12-15, 18-19

Figs. 16-17

Figs. 25-30

Fig. 31

Figs. 35-36

Figs. 37-39

Figs. 40-41

Figs. $42-43$

Fig. 50

Figs. 51-53
Psammothidium bioretii (Germain) Bukhtiyarova \& Round Psammothidium chlidanos (Hohn \& Hellerman) Lange-Bertalot Psammothidium daonense (Lange-Bertalot) Lange-Bertalot Achnanthes sp. No. 3 Posets

Psammothidium marginulatum (Grunow) Bukhtiyarova et Round Psammothidium acidoclinatum (Lange-Bertalot) Lange-Bertalot Psammothidium rossii (Hustedt) Bukhtiyarova et Round Achnanthes sp. 7 Pixón Psammothidium cf. daonense (Lange-Bertalot) Lange-Bertalot Psammothidium ventralis (Krasske) Bukhtiyarova et Round Achnanthes ziegleri Lange-Bertalot

Lake Llebreta, sediment PYR58

Lake Posets, sediment PYR42

Lake Urdiceto, sediment PYR125

Lake Les Laquettes, sediment PYR27

Lake Siscar, sediment PYR126

Lake Monges, sediment PYR57

Lake Cap Long, sediment PYR24

Lake Bleu de Rabassoles, sediment PYR112

Lake Senó, epilithic EpiPYR84

Lake Estelat, sediment PYR120

Lake Pixón, sediment PYR44

Lake Blaou, sediment PYR94

Lake Lliterola, epilithic EpiPYR33

Lake Laurenti, sediment PYR111

Lake Acherito, sediment PYR01 

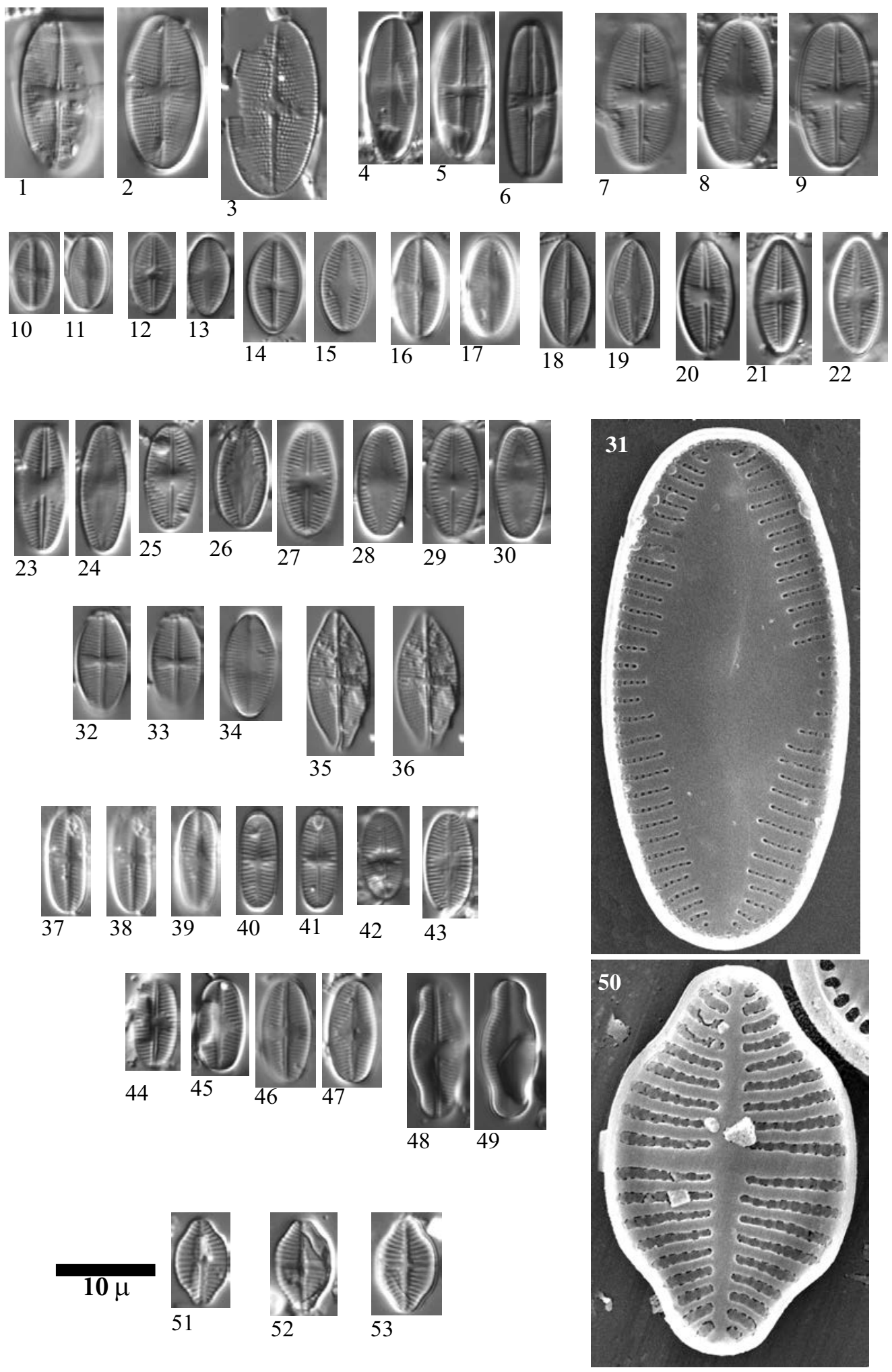

Limnetica, 36 (1): 127-395 (2017) 
Plate $52 \quad$ LM: $x 1500$

SEM: $\mathrm{x} 4500$

Figs. 1-3, 7-9 Cocconeis euglyptoides (Geitler) Lange-Bertalot

Fig. 4

Cocconeis cf. neothumensis Krammer

Fig. 5-6

Cocconeis neodiminuta Krammer

Figs. 1-2 Lake Sen, sediment PYR40

Fig. 3

Figs. 4-5

Lake Estom, sediment PYR15

Fig. 6

Lake Laurenti, epilithic EpiPYR111

Fig. 7

Lake Acherito, sediment PYR01

Fig. 8

Lake Roumassot, epilithic EpiPYR04

Fig. 9

Lake Arnales, epilithic EpiPYR09

Lake Roumassot, sediment PYR04 

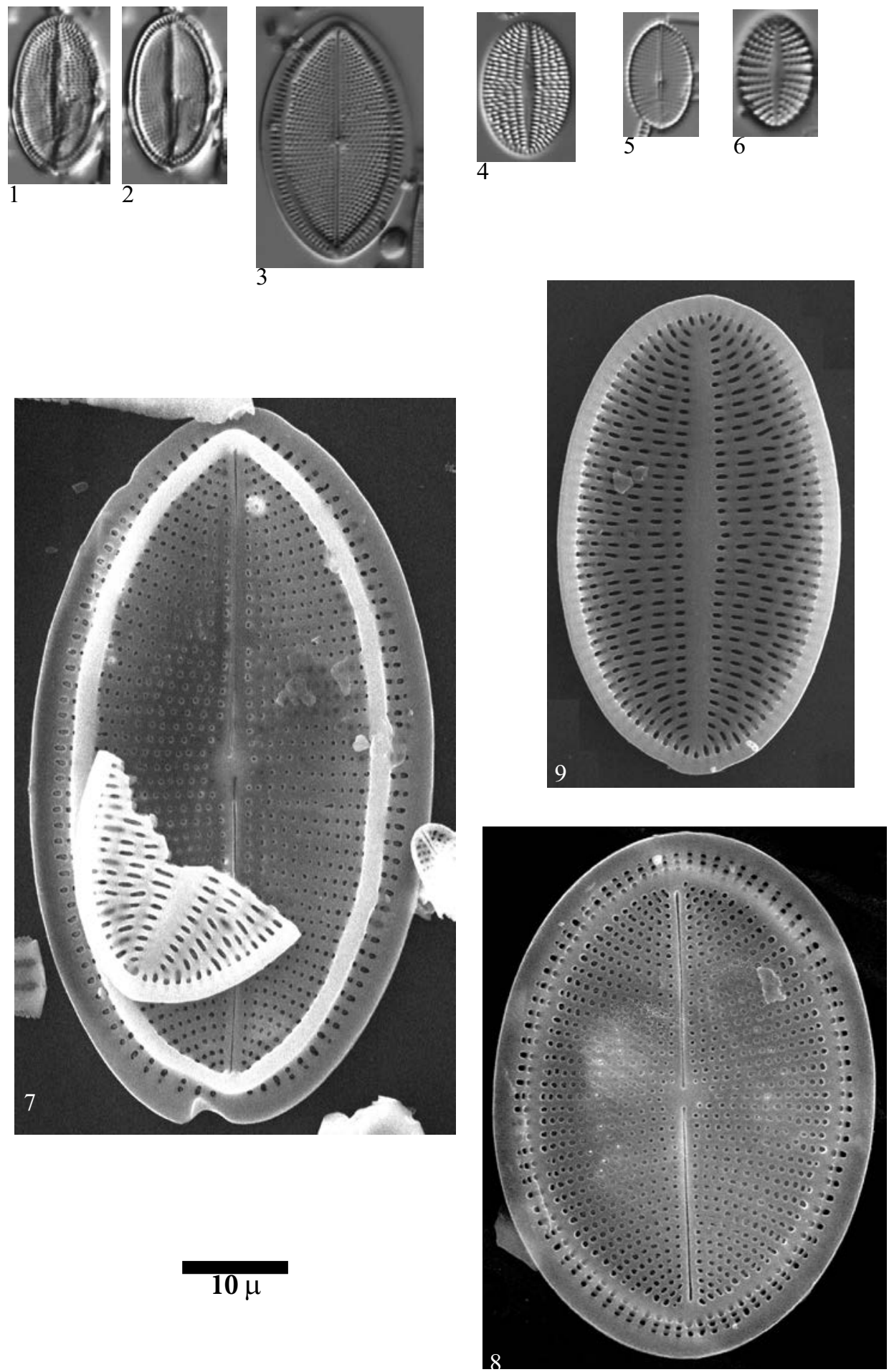
Figs. 1-6

Figs. 7-8

Figs. 9-19

Figs. 20-23

Figs. 24-31

Figs. 32-33

Figs. 34-36

Figs. 1-2, 5-6

Figs. 3, 7-9

Figs. 4, 10-13, 19 35-36

Figs. 14, 20-22, 24-31

Figs. $15,17-18$

Fig. 16

Fig. 23

Fig. 32

Fig. 33

Fig. 34
Navicula caterva Hohn \& Hellerman

Navicula cf. caterva Hohn \& Hellerman

Navicula cryptocephala Kützing

Navicula cf. cryptocephala Kützing

Navicula wildii Lange-Bertalot

Navicula cf. moskalii Metzeltin, Witkowski \& Lange-Bertalot

Navicula cf. cryptocephala Kützing

Lake Sen, sediment PYR40

Lake Arnales, sediment PYR09

Lake Posets, sediment PYR42

Lake Arratille, sediment PYR11

Lake Acherito, sediment PYR01

Lake Col d'Arratille, sediment PYR12

Lake Mes Amunt de Tristaina, sediment PYR86

Lake Helado del Monte Perdido, sediment PYR19

Lake Tourrat, sediment PYR23

Lake Arnales, epilithic EpiPYR09 


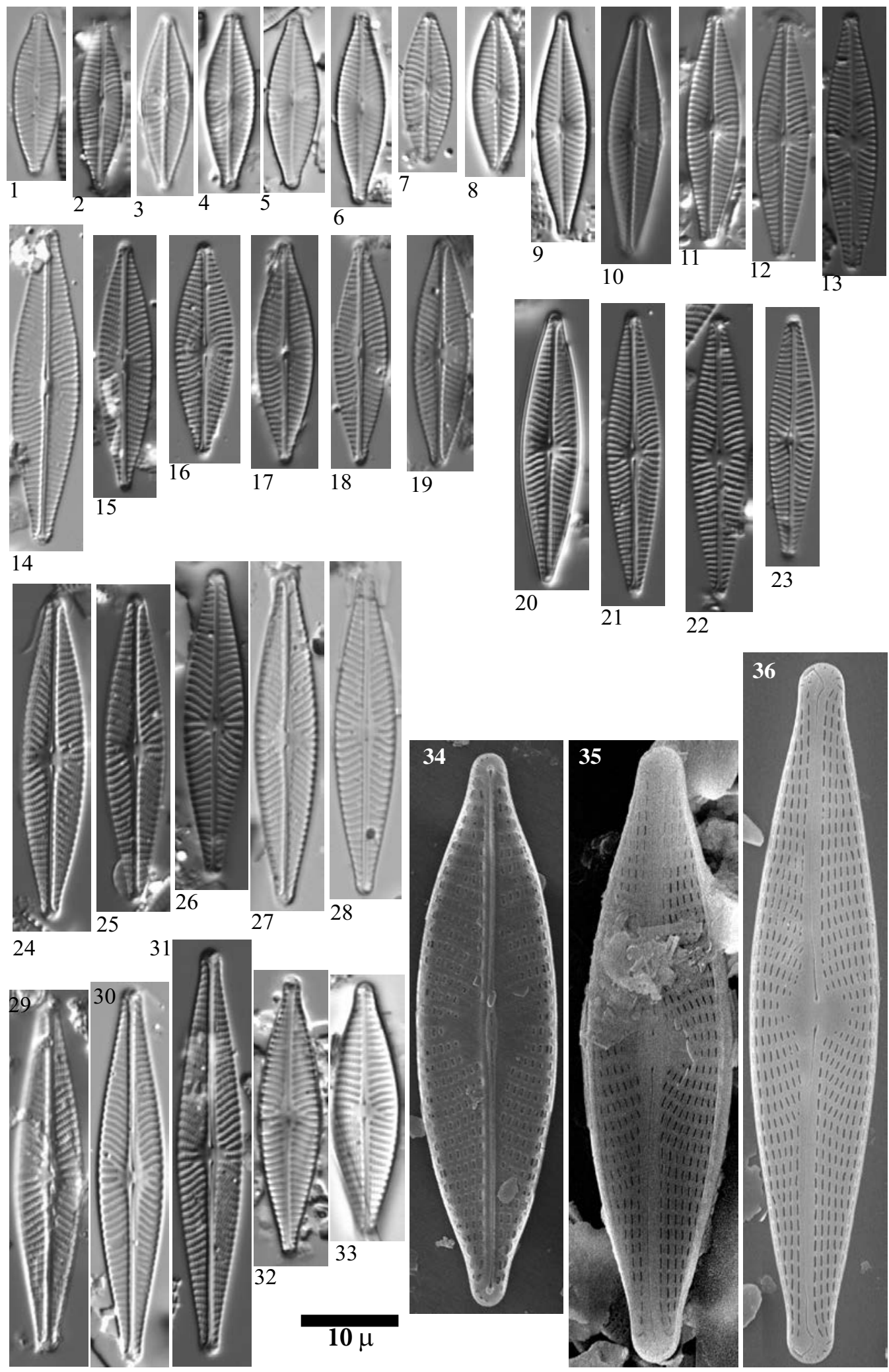


SEM: $x 5000$

Figs. 1-15

Figs. 16-19

Figs. 20-22

Figs. 23-24

Figs. 25-27

Figs. 1-6, 8-10, 12, 15

Fig. 7

Figs. 13-14

Fig. 16

Figs. 17-19

Fig. 20

Fig. 11

Fig. 21

Fig. 22

Fig. 23

Fig. 24

Fig. 25

Figs. 26-27
Navicula cryptotenella Lange-Bertalot

Navicula heimansioides Lange-Bertalot

Navicula exilis Kützing

Navicula notha Wallace

Navicula cryptofallax Lange-Bertalot \& Hofmann
Lake Arratille, sediment PYR11

Lake Sen, sediment PYR40

Lake Col d'Arretille, sediment PYR12

Lake Gelat Bergús, sediment PYR65

Lake Bleu de Rabassoles, sediment PYR112

Lake Llosás, sediment PYR46

Lake Port Bielh, sediment EpiPYR28

Lake Baiau Superior, sediment PYR76

Lake Trebens, sediment PYR114

Lake Argonella, sediment PYR78

Lake Mes Amunt de Tristaina, sediment PYR86

Lake Burg

Lake Acherito, sediment PYR01 

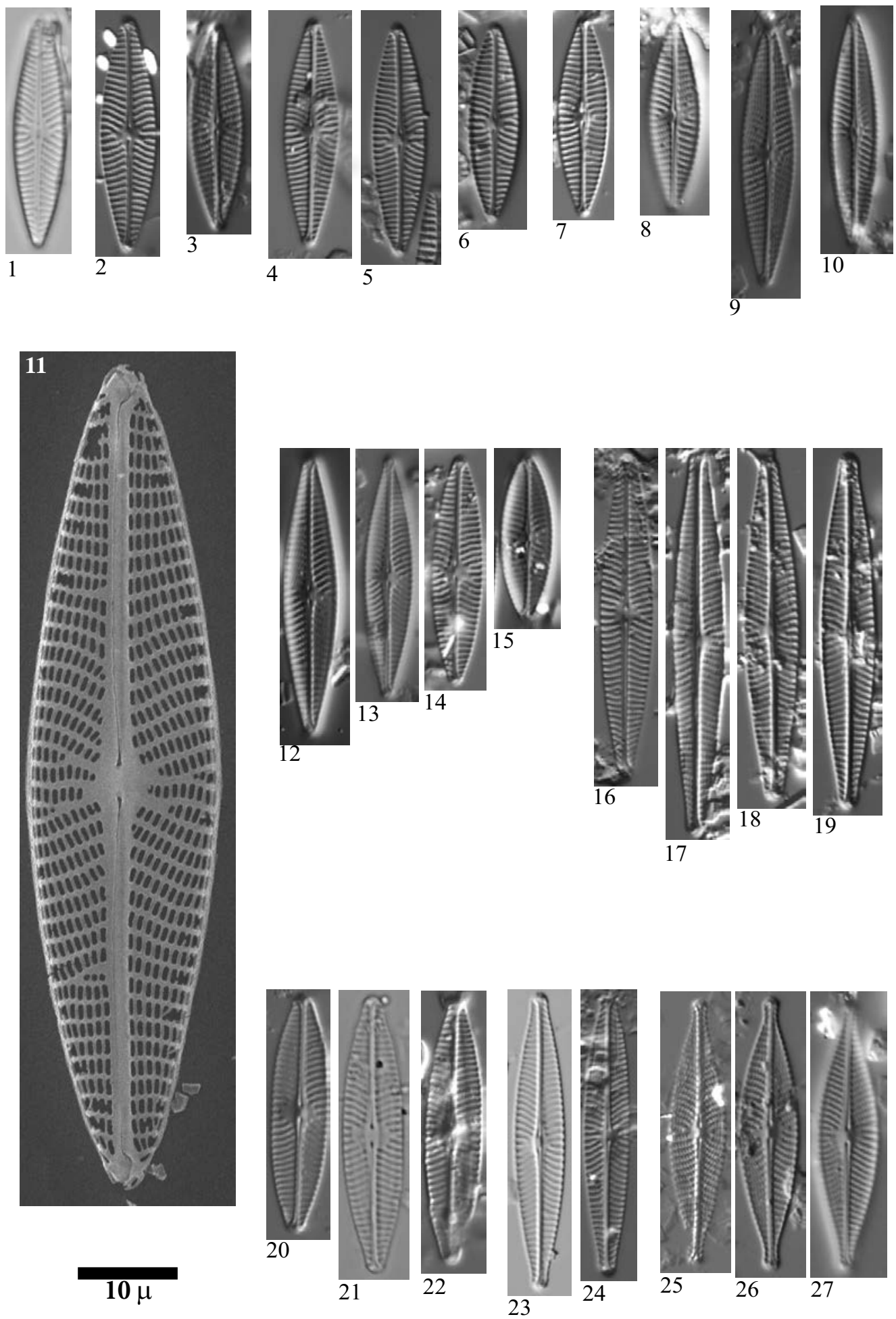
SEM: $\mathrm{x} 4000$

Figs. 1, 8-13

Figs. 2-6

Fig. 7

Figs. 14-22

Figs. 23-26

Figs. 27-28

Fig. 29

Figs. 30-31

Fig. 1

Figs. 2, 6

Fig. 3

Figs. 4-5, 12

Figs. 7, 13

Figs. 8, 10-11, 24-26

Fig. 9

Figs. 14-16, 23, 27-28

Fig. 17

Figs. 18-20

Figs. 21-22

Fig. 29

Figs. 30-31
Navicula catalanogermanica Lange-Bertalot \& Hofmann

Navicula cf. antonii Lange-Bertalot \& Rumrich

Navicula cf. upsaliensis (Grunow) Peragallo

Navicula pseudolanceolata Lange-Bertalot

Navicula trophicatrix Lange-Bertalot

Navicula subalpina Reichardt

Navicula cf. libonensis Schoeman

Navicula sp. No. 9 Arratille

Lake Laurenti, sediment PYR111

Lake Tourrat, sediment PYR23

Lake Cap Long, sediment PYR24

Lake Acherito, sediment PYR01

Lake Barroude Inf., sediment PYR29

Lake Col d'Arratille, sediment PYR12

Lake Helado del Monte Perdido, epilithic EpiPYR19

Lake Arratille, sediment PYR11

Lake Montagnon, sediment PYR121

Lake Arnales, sediment PYR09

Lake Roumassot, sediment PYR04

Lake Burg, sediment BURG 1195

Lake Arratille, epilithic EpiPYR11 

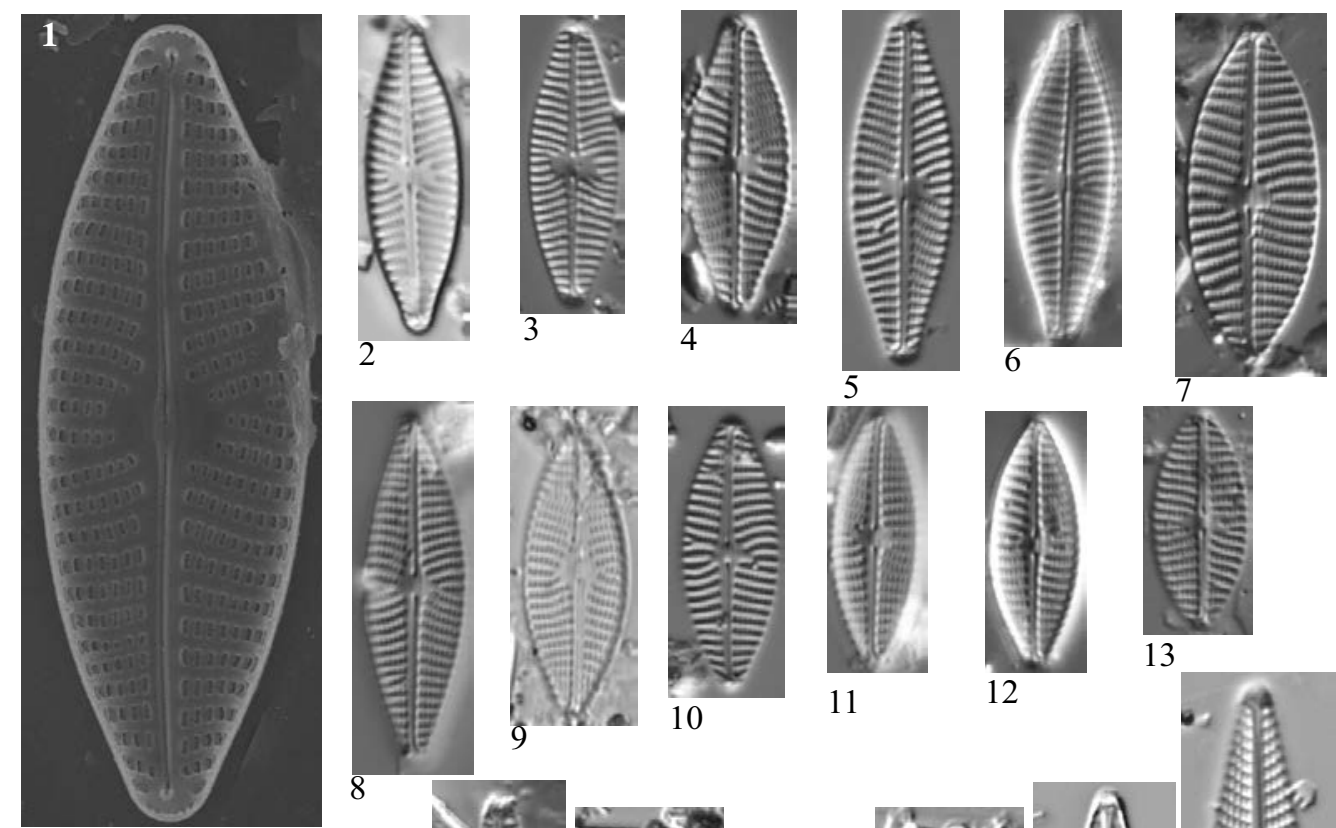

$$
8
$$
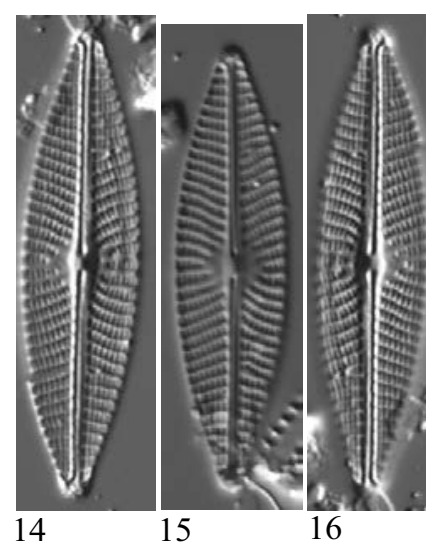

1.

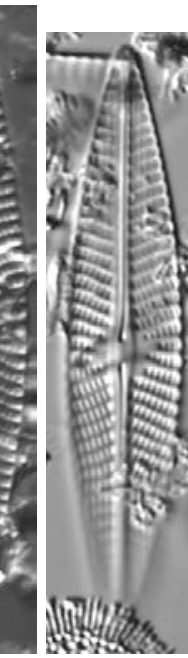

18
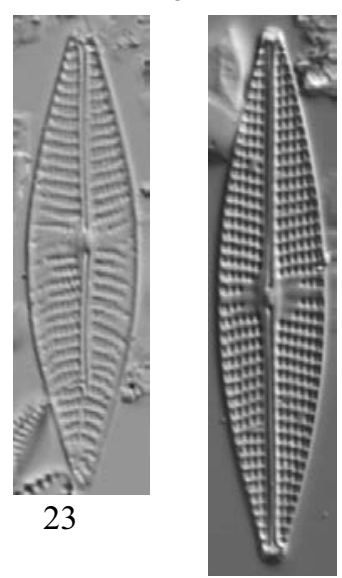

24
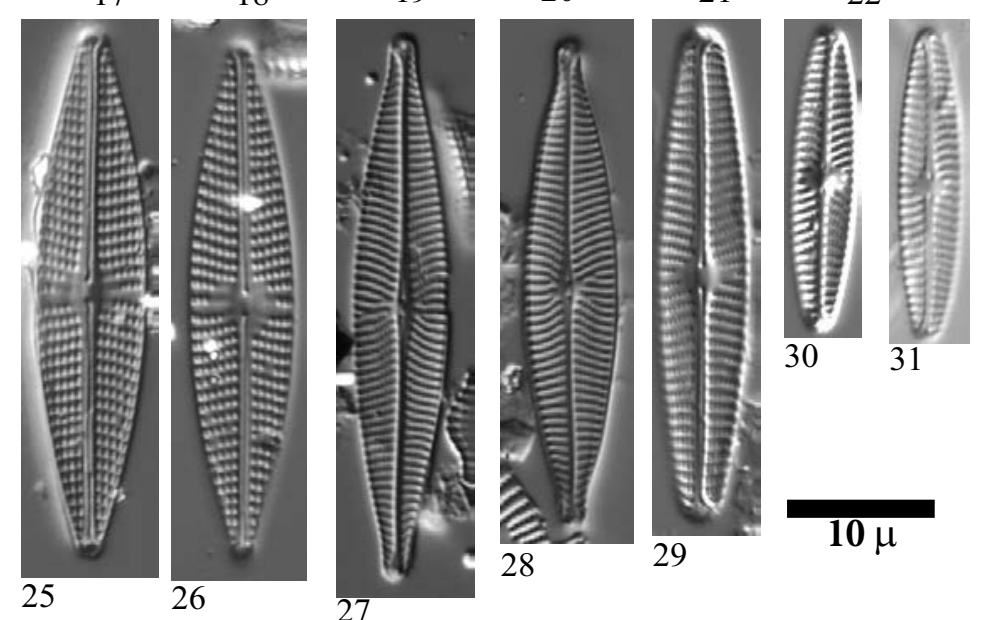

Limnetica, 36 (1): 127-395 (2017) 
Plate $56 \quad$ LM: $x 1500$

SEM: $\times 3000$

Figs. 1-4 Navicula cf. oligotraphenta Lange-Bertalot \& Hofmann

Figs. 5-6 Navicula cf. trivialis Lange-Bertalot

Figs. 7-8 Navicula sp.

Figs. 9-10 Navicula viridula Kützing

Fig. 1 Lake Burg, BURG $1195 \mathrm{~cm}$

Fig. 2 Lake Basa de la Mora, sediment PYR32

Figs. 3-4 Lake Laurenti, sediment PYR111

Figs. 5-6 Lake Burg, sediment BURG 1068

Fig. 7 Lake Burg, sediment BURG 1072

Fig. 8 Lake Burg, sediment BURG 913

Figs. 9-10 Lake Burg, sediment BURG 843 

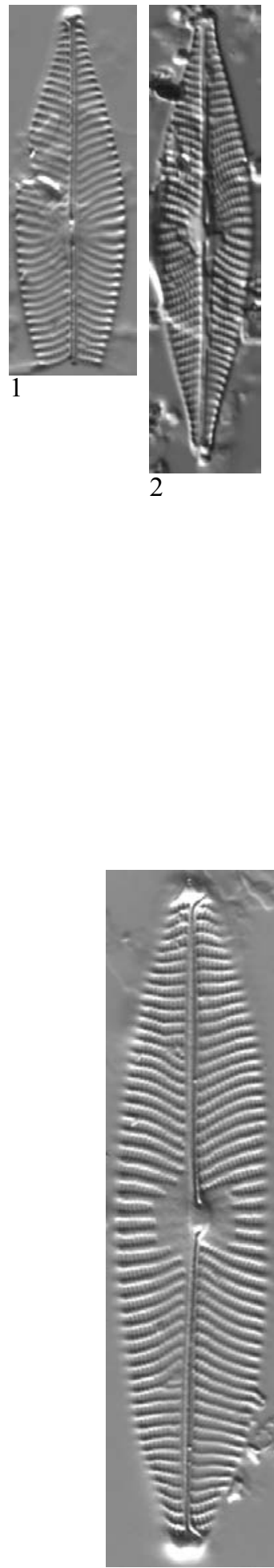

9

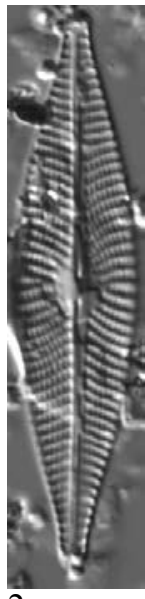

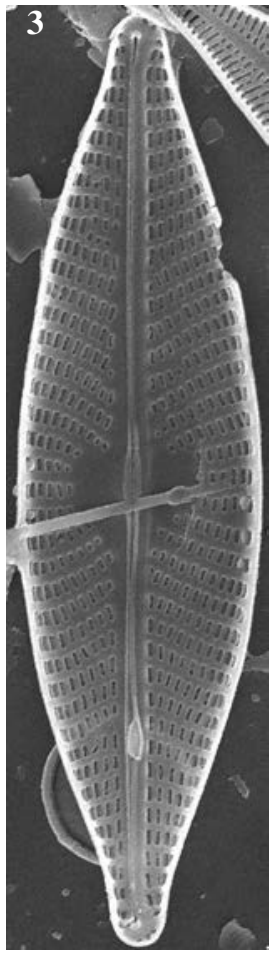
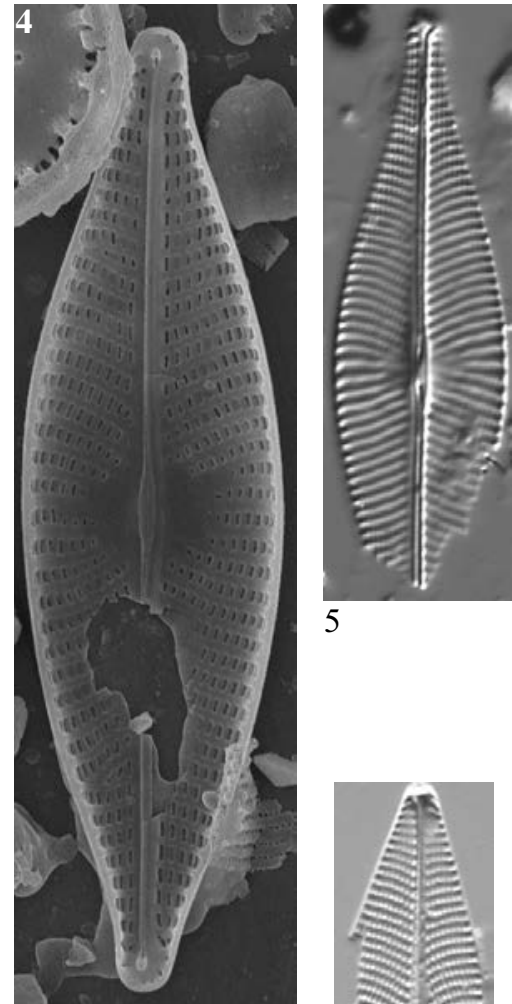

5
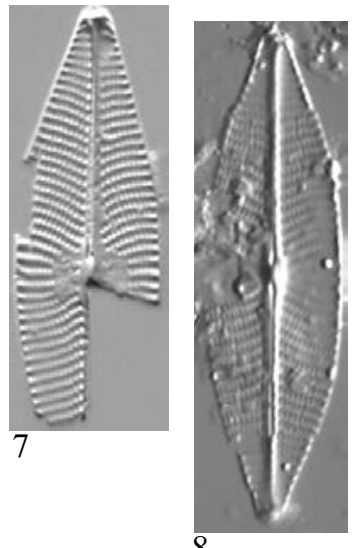

8

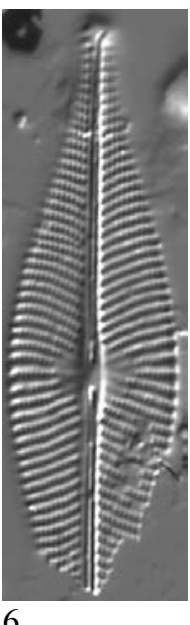

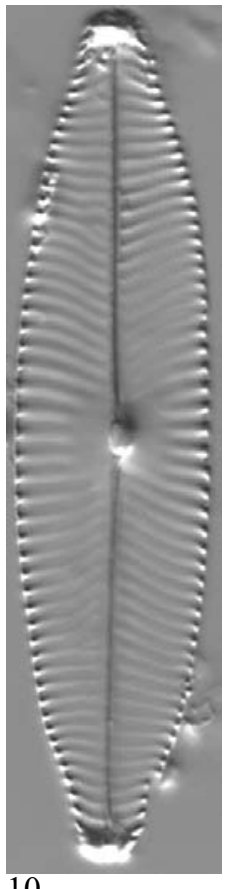

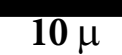


Plate $57 \quad$ LM: $x 1500$

SEM: $\mathrm{x} 2500$

Figs. 1-2 Navicula vulpina Kützing

Figs. 3-7 Navicula radiosa Kützing

Figs. 1-2, $7 \quad$ Lake Arratille, sediment PYR11

Fig. 3 Lake Gran de la Pera, sediment PYR102

Fig. $4 \quad$ Lake Plan, sediment PYR69

Fig. 5 Lake Sen, sediment PYR40

Fig. 6 Lake Posets, sediment PYR42 

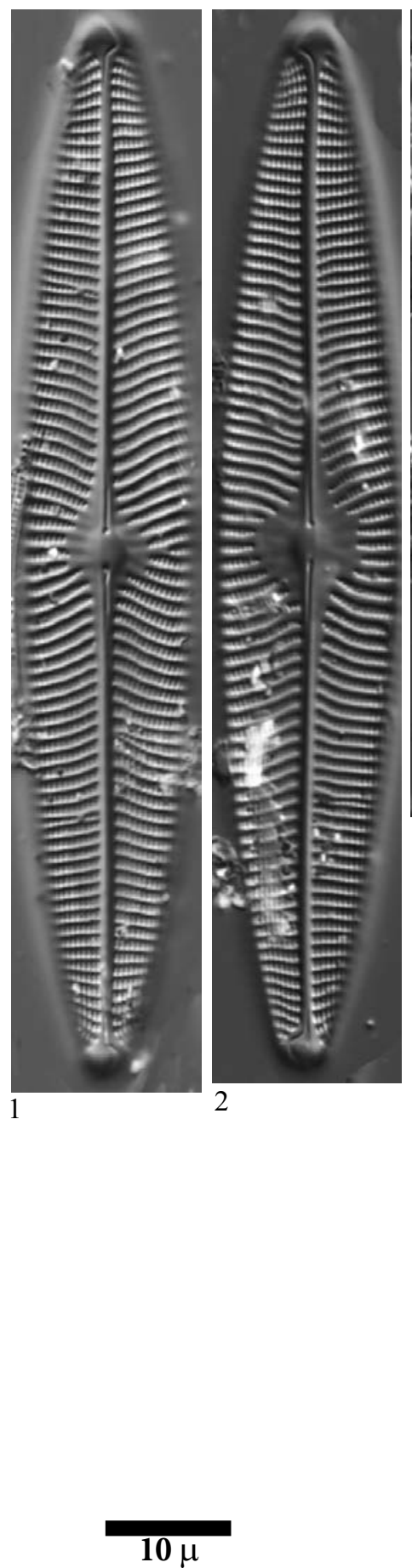

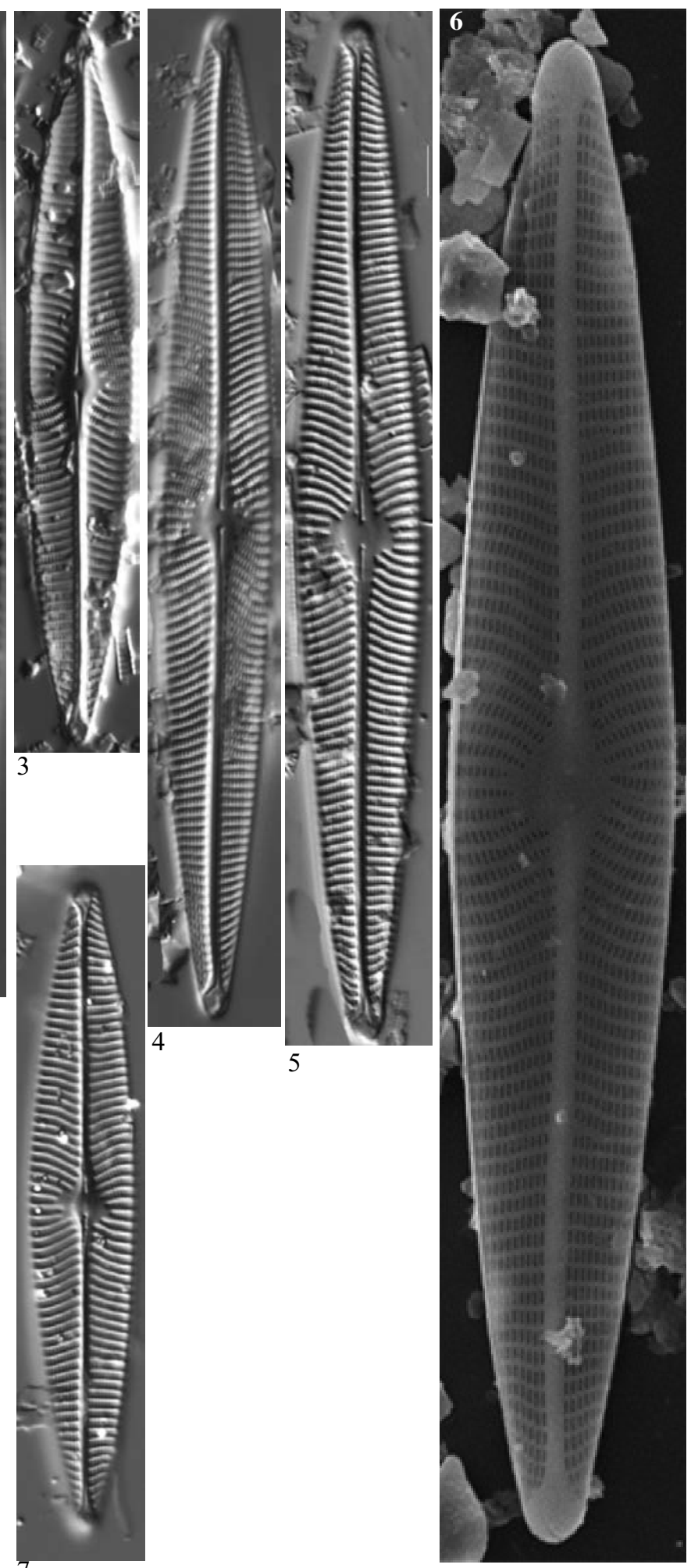


Plate $58 \quad$ LM: $x 1500$

SEM: $x 10000$

Figs. 1-2 Navicula venerablis Hohn \& Hellerman

Figs. 3-7 Navicula angusta Grunow

Fig. 1 Lake Coronas, sediment PYR47

Figs. 2-3 Lake Redon, sediment REDOM

Figs. 4-5, 7 Lake Mariola, sediment PYR80

Fig. $6 \quad$ Lake Angonella, epilithic EpiPYR78 

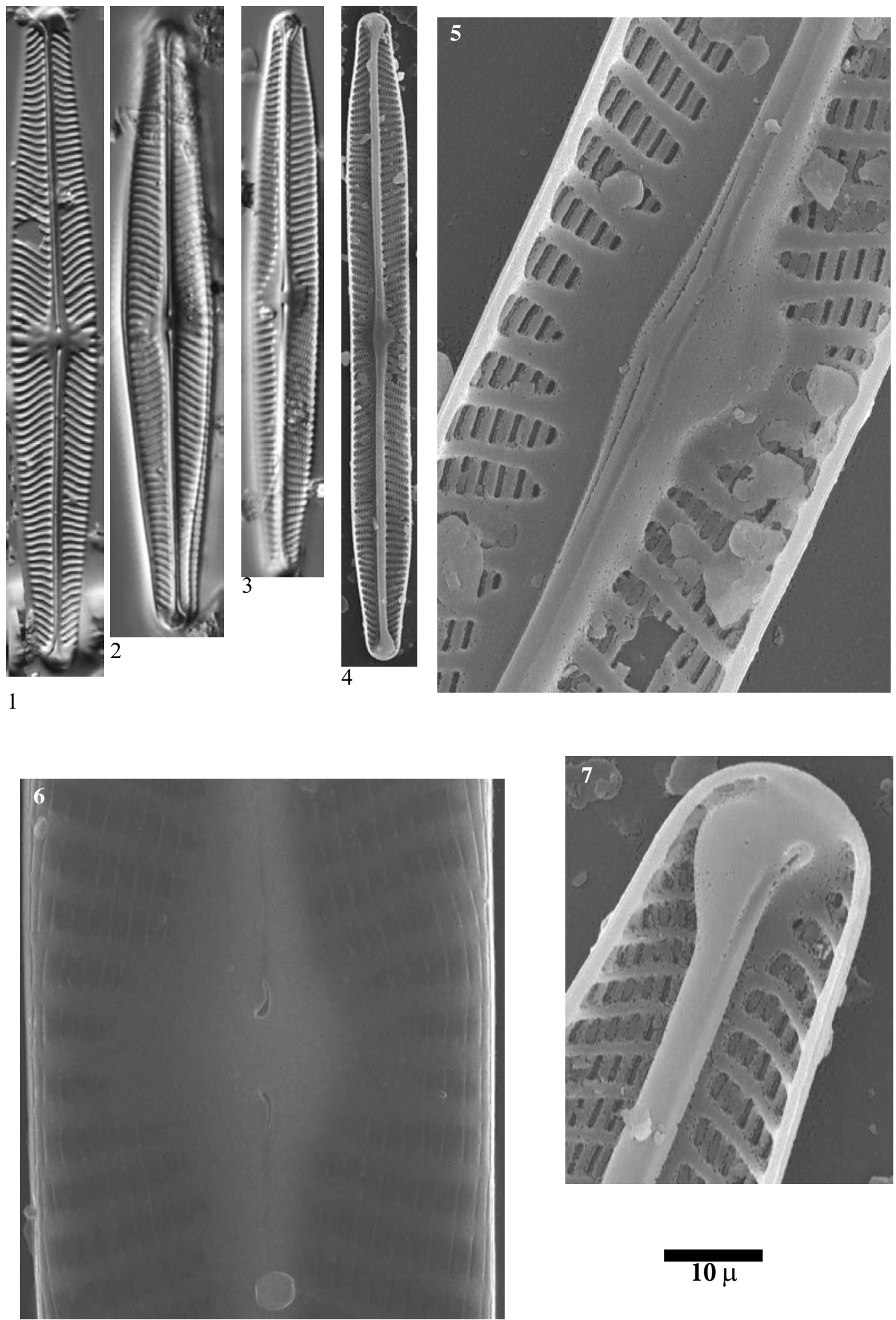
Plate 59

LM: x1500

SEM: Figs. 10-11 x5000, Figs. 19-20 x10000, Figs. 21-22

$\mathrm{x} 4000$

Figs. 1-11

Sellaphora disjuncta (Hustedt) D. G. Mann

Figs. 12-22

Sellaphora laevissima (Kützing) D. G. Mann

Figs. 1, 5, 7, 13, 18 Lake Posets, sediment PYR42

Fig. 2

Lake Burg, sediment BURG 1062

Figs. 3-4, 6

Lake Inferior de la Gallina, sediment PYR87

Figs. 8-9

Lake Llebreta, sediment PYR58

Figs.10-11, 14

Lake Burg

Fig. 12

Lake Arratille, sediment PYR11

Fig. 15

Lake Burg, sediment BURG 953

Fig. 16

Lake Burg, sediment BURG 543

Fig. 17

Lake Col d Arratille, sediment PYR12 

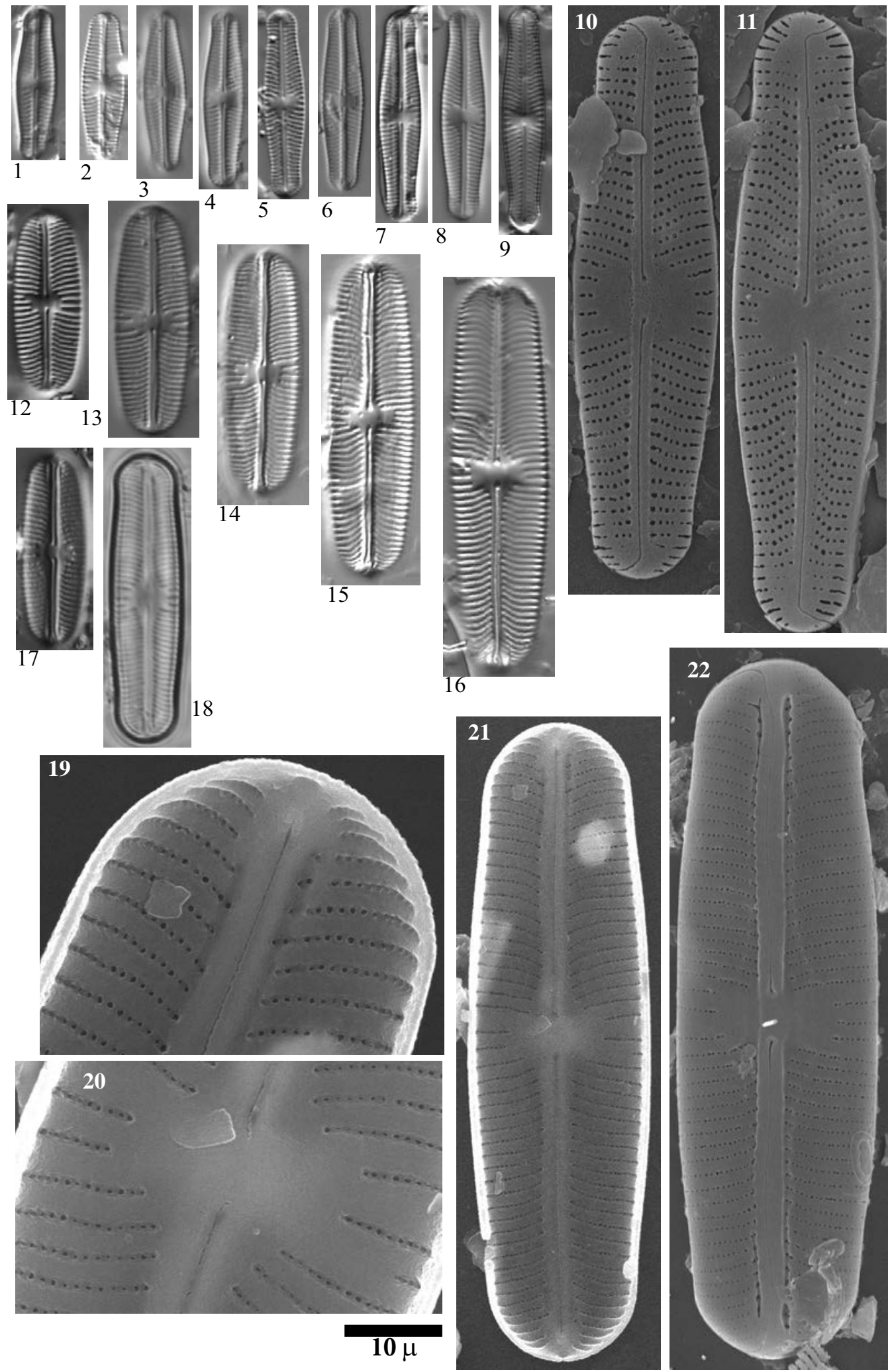

Limnetica, 36 (1): 127-395 (2017) 
Plate $60 \quad$ LM: $x 1500$

SEM: Fig. 3 x9000, Fig. 4 x4000, Fig. 5 x3500, Fig. 6 x10000

Figs- 1-6 Sellaphora bacillum (Ehrenberg) D. G. Mann

Fig. $1 \quad$ Lake Arratille, sediment PYR11

Figs. 2, 3-6 Lake Laurenti, sediment PYR111 

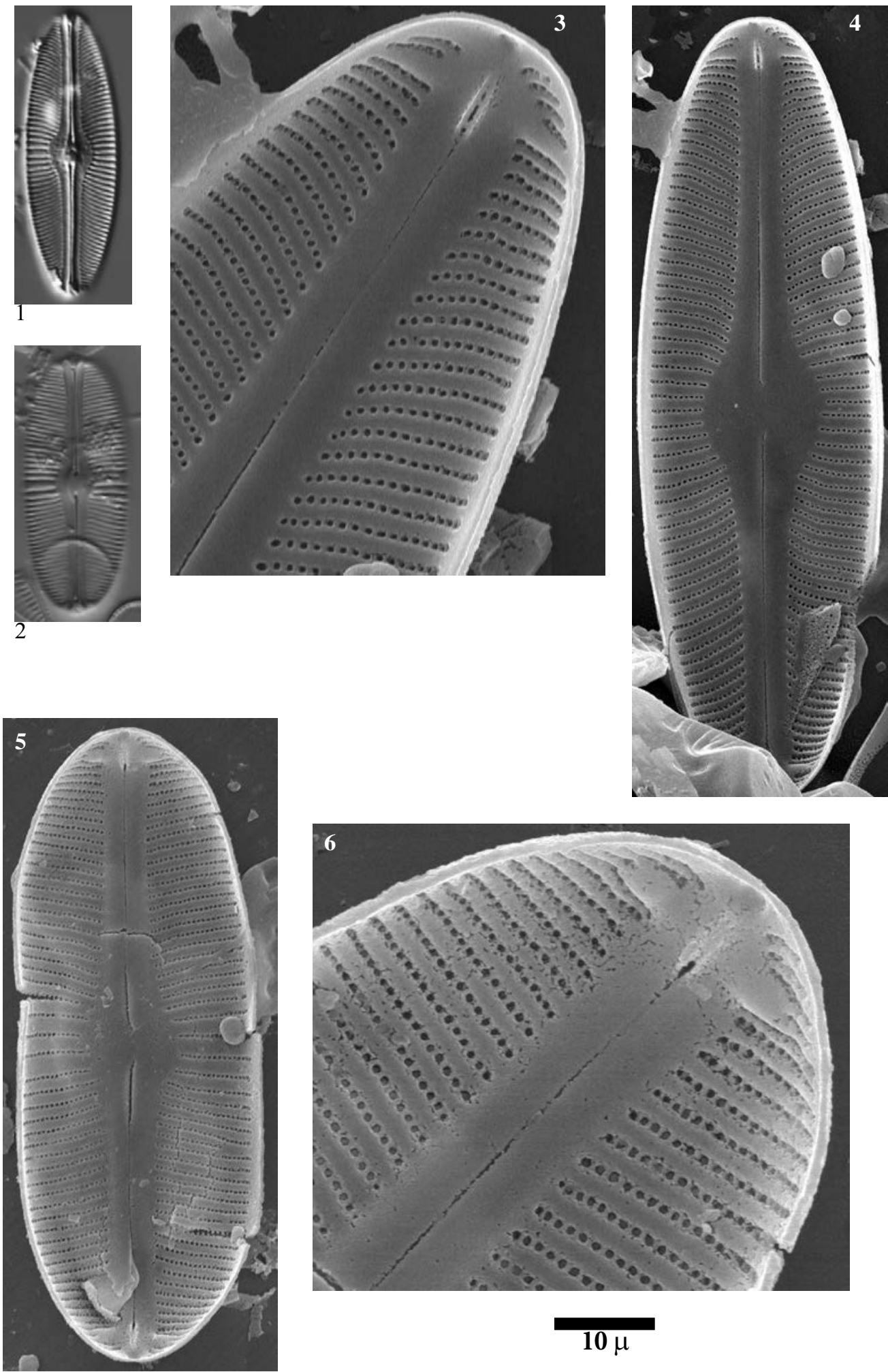

Limnetica, 36 (1): 127-395 (2017) 
SEM: x6000

Fig. 1

Figs. 2-11

12-14

Fig. 15

Fig. 1

Figs. 2, 6-7, 9-11

Figs. 3, 5

Fig. 4

Fig. 8

Fig. 12

Fig. 13

Fig. 14

Fig. 15
Sellaphora pupula (Kützing) Mereschkowsky sensu lato cf. Sellaphora blackfordensis Mann \& Droop

Sellaphora pseudopupula (Krasske) Lange-Bertalot

Sellaphora pupula (Kützing) Mereschkowsky sensu lato

Lake Burg

Lake Posets, sediment PYR42

Lake Albe, sediment PYR96

Lake Arratille, sediment PYR11

Lake Burg, sediment BURG 973

Lake Angonella, epilithic EpiPYR78

Lake Garbet, sediment PYR81

Lake Laurenti, sediment PYR111

Lake Acherito, sediment PYR01 


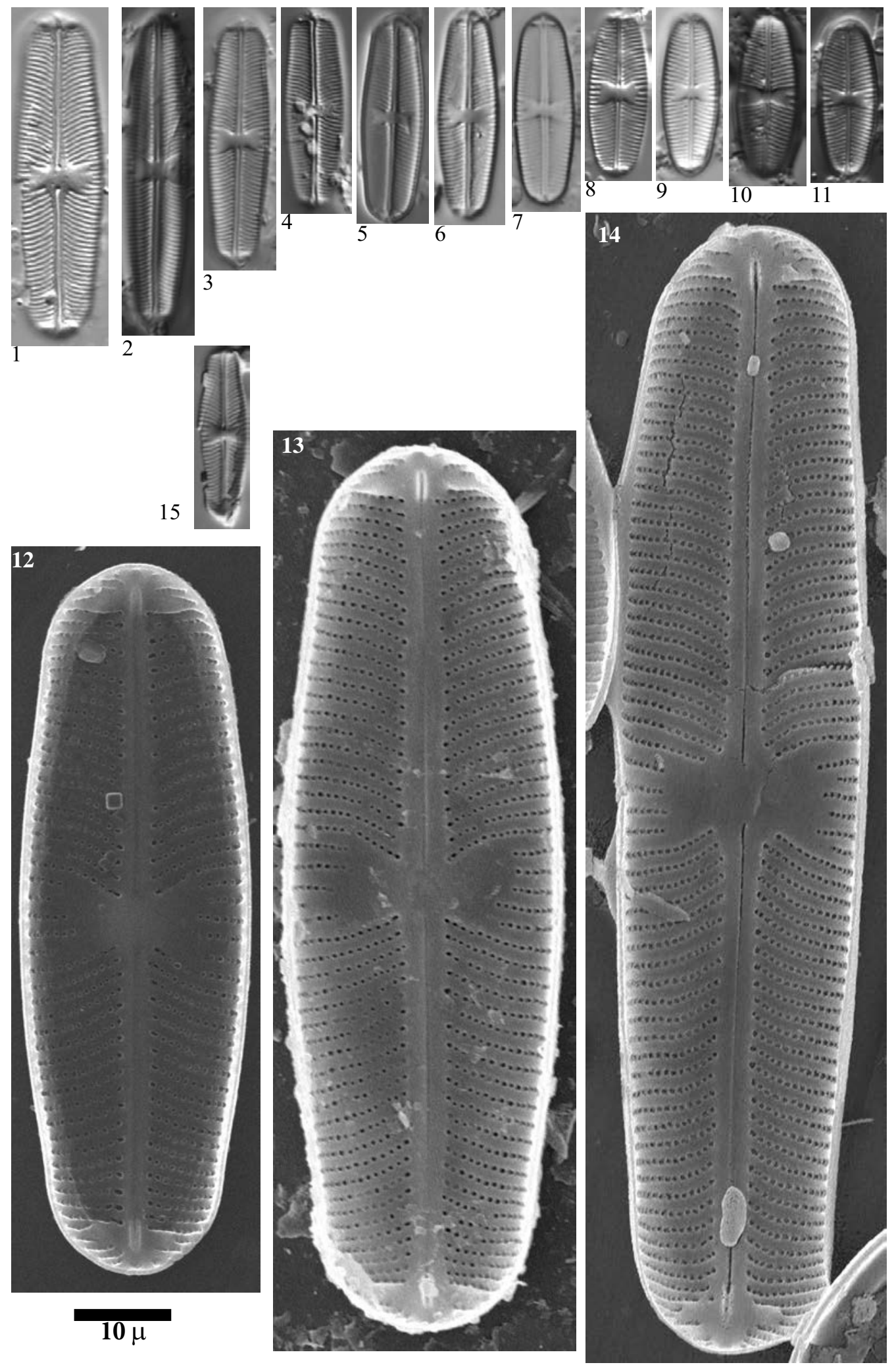


Plate $62 \quad$ LM: $x 1500$

SEM: Figs. 3, 10 x5000, Fig. 11 x 4000, Fig. 12 x6000

Figs. 1-3 Sellaphora stroemii (Hustedt) Kobayasi

Figs. 4-6, $10 \quad$ Sellaphora pupula (Kützing) Mereschkowsky sensu lato cf. Sellaphora auldreekie Mann \& McDonald

Figs. 7-8 Sellaphora pupula (Kützing) Mereschkowsky sensu lato cf. Sellaphora capitata Mann \& McDonald

Fig. $9 \quad$ Sellaphora sp. No. 1 Ensangents

Fig. $11 \quad$ Sellaphora pupula (Kützing) Mereschkowsky

Fig. 12 Sellaphora aff. nanoides Lange-Bertalot, Cavacini, Tagliaventi \& Alfinito

Fig. 1 Lake Basa de la Mora, sediment PYR32

Fig. 2 Lake Gran de Mainera, sediment PYR70

Fig. 3 Lake Port Bielh, epilithic EpiPYR28

Fig. $4 \quad$ Lake Burg, sediment BURG 927

Fig. $5 \quad$ Lake Burg, sediment BURG 926

Fig. $6 \quad$ Lake Arratille, sediment PYR11

Fig. $7 \quad$ Lake Burg, sediment BURG 774

Fig. 8 Lake Burg, sediment BURG 782

Fig. 9 Lake Ensangents Sup., sediment PYR106

Figs. 10-11 Lake Laurenti, sediment PYR111

Fig. 12 Lake Gros de Camporrells, sediment PYR110 

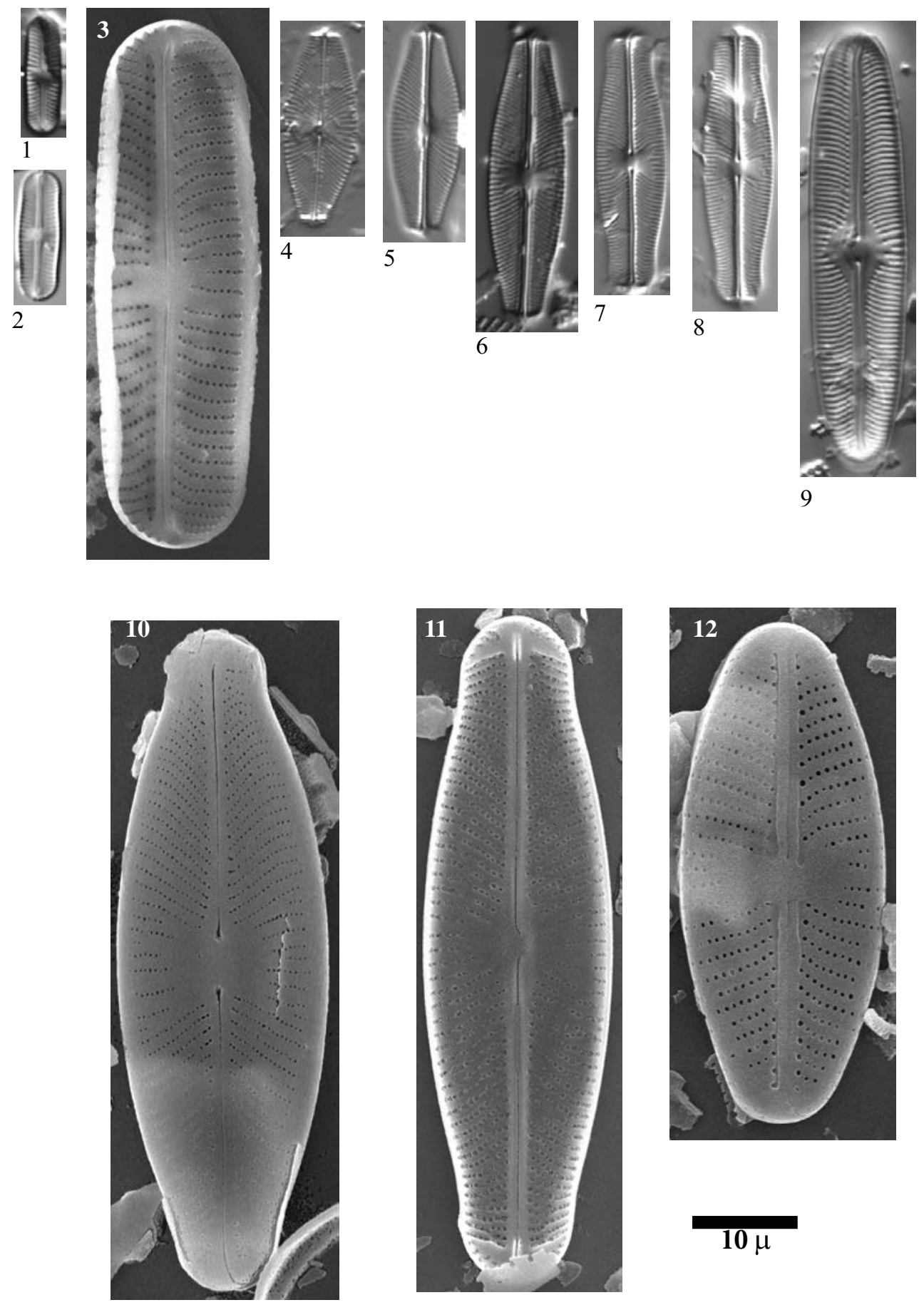
Plate $63 \quad$ LM: $x 1500$

SEM: Fig. 18 x6000, Fig. 19 x8000

Figs. 1-2

Figs. 3-10

Figs. 11-19

Fig. 20

Figs. 1-2

Fig. 3

Figs. 4, 6-7, 11-12

Fig. 5

Figs. 8-10

Fig. 13

Fig. 14

Fig. 15

Fig. 16

Fig. 17

Fig. 18

Fig. 19

Fig. 20
Cavinula mollicula (Hustedt) Lange-Bertalot

Cavinula cocconeiformis (Gregory ex Greville) Mann \& Stickle sensu lato

Cavinula pseudoscutiformis (Hustedt) Mann \& Stickle

Navicula sp. No. 8 Sotllo
Lake Negre, sediment PYR108

Lake Laurenti, sediment PYR111

Lake Inferior de la Gallina, sediment PYR87

Lake Garbet, sediment PYR81

Lake Blaou, sediment PYR94

Lake Port Bielh, sediment PYR28

Lake Arratille, sediment PYR11

Lake Llebreta, sediment PYR58

Lake Les Laquettes, sediment PYR27

Lake Burg, sediment BURG 1187

Lake Burg

Lake Gros de Camporrells, sediment PYR110

Lake Sotllo, epilithic PYR89 

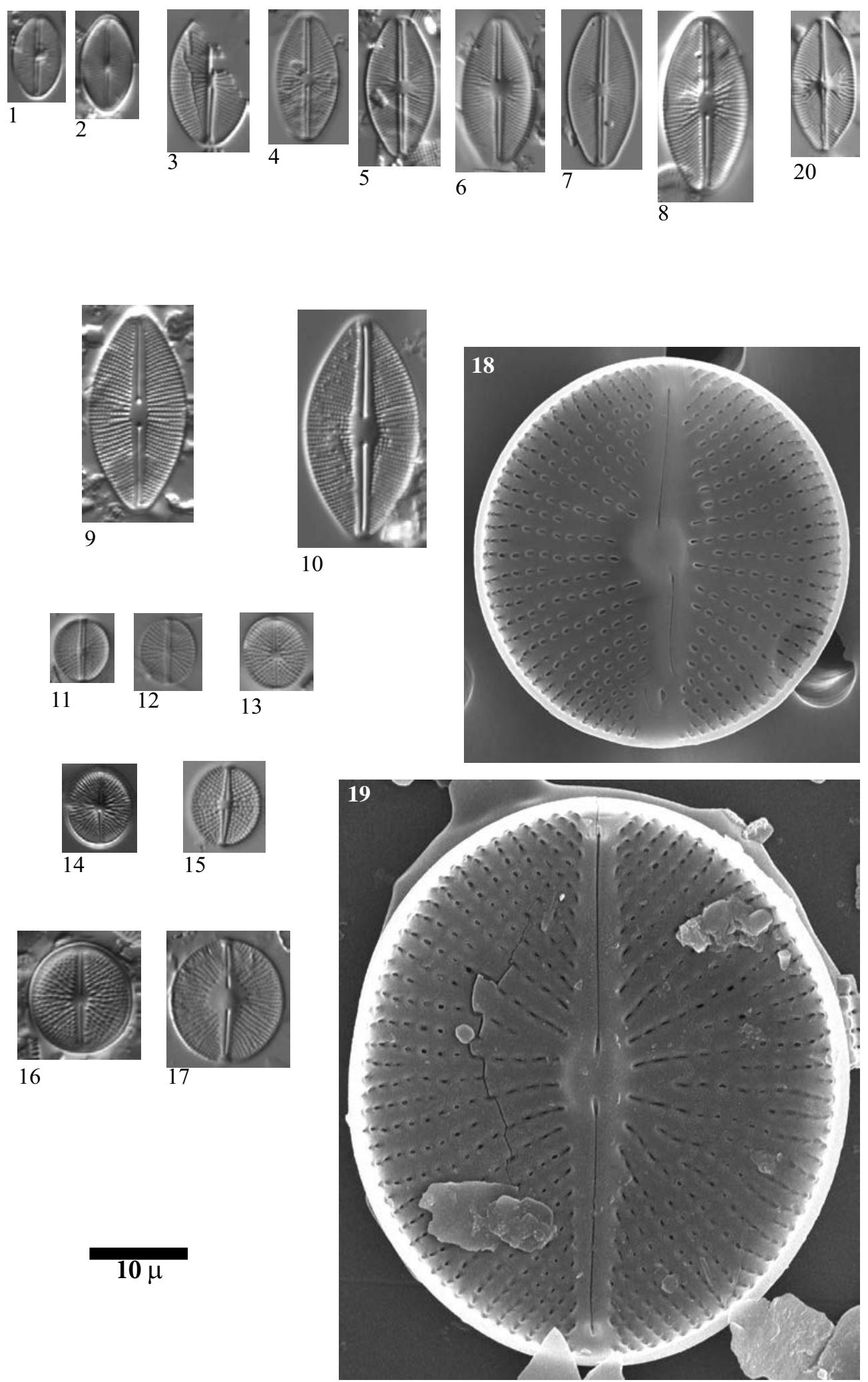

Limnetica, 36 (1): 127-395 (2017) 
Plate $64 \quad$ LM: $x 1500$

SEM: Fig. 7 x4000, Fig. 17 x7000

Figs. 1-4 Placoneis ignorata (Schimanski) Lange-Bertalot

Figs. 5-7,9 Placoneis explanata (Hustedt) Lange-Bertalot

Fig. 8 Placoneis symmetrica (Hustedt) Lange-Bertalot

Fig. $10 \quad$ Placoneis sp. No. 1 Acherito

Figs. 11-13 Placoneis elginensis (Gregory) Cox sensu lato

Figs. 14-15 Placoneis sp. No. 3 Burg

Fig. 16 Placoneis cf. abiskoensis (Hustedt) Lange-Bertalot et Metzeltin

Fig. $17 \quad$ Placoneis paraelginensis Lange-Bertalot

Figs. 1, 3 Lake Negre, sediment PYR96

Fig. 2 Lake Burg, sediment BURG 480

Fig. $4 \quad$ Lake Burg

Fig. 5-6 Lake Burg, sediment BURG 543

Fig. $7 \quad$ Lake Blaou, sediment PYR27

Figs. 8, $10 \quad$ Lake Port Bielh, sediment PYR01

Fig. 11 Lake Burg, sediment BURG 1053

Fig. 12 Lake Burg, sediment BURG 1007

Fig. 13 Lake Burg, sediment BURG 845

Fig. $14 \quad$ Lake Burg, sediment BURG 1031

Fig. 15 Lake Burg, sediment BURG 848

Fig. 16 Lake Burg, sediment BURG 1104

Fig. 17 Lake Burg, sediment BURG 425 

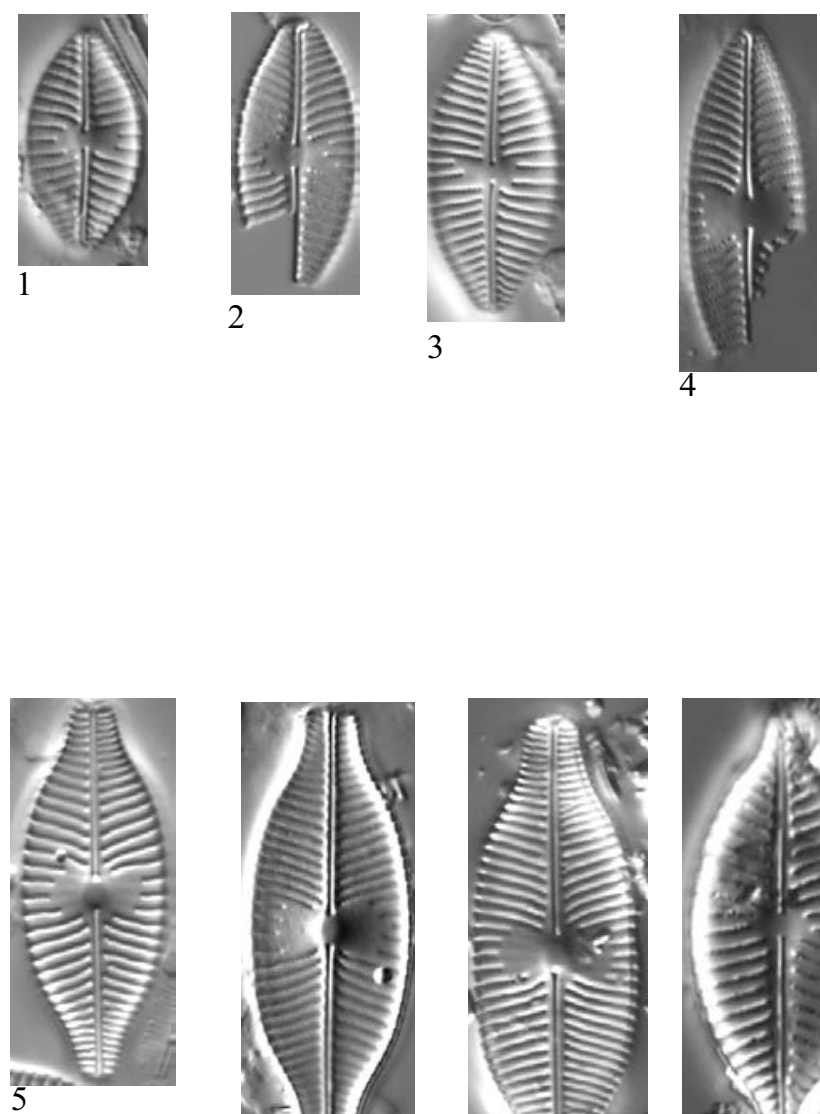

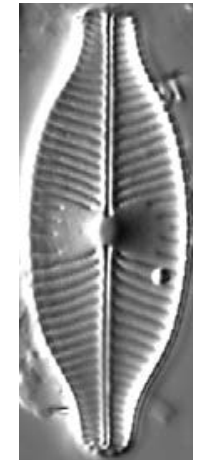

6
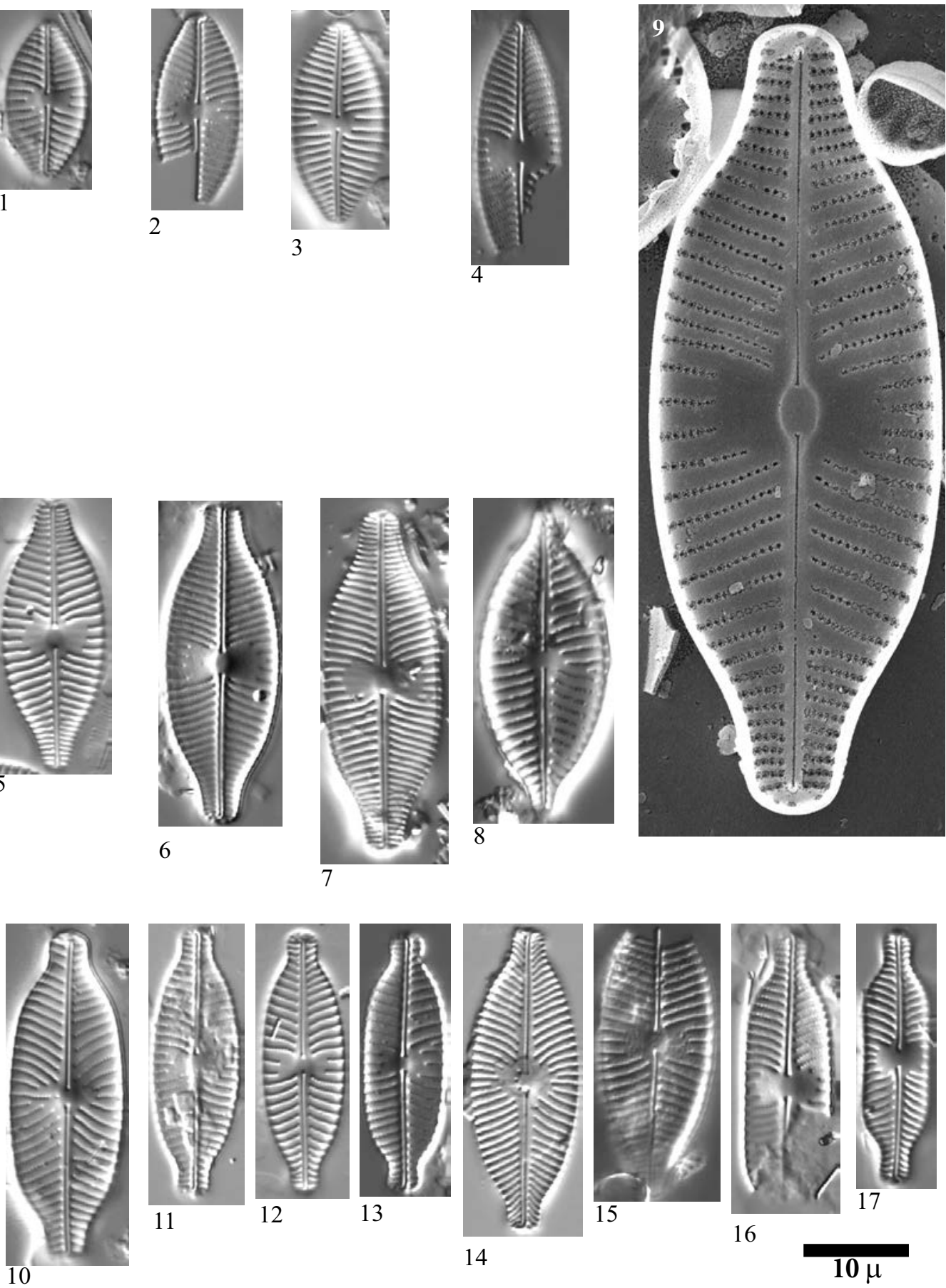
Plate 65 LM: $x 1500$

SEM: Fig. $17 \times 7000$

Fig. 1-4, $8 \quad$ Navicula sp. No. 2 Liat

?5-7

Figs. 9-16 Navicula detenta Hustedt

Figs. 1, 3-5, Lake Negre, sediment PYR42

10-13

Fig. 2

Lake Negre, sediment PYR55

Figs. 6-9

Lake Negre, sediment PYR40

Fig. 8

Lake Negre, sediment PYR80

Figs. 14-16 Lake Negre, epilithic EpiPYR78 

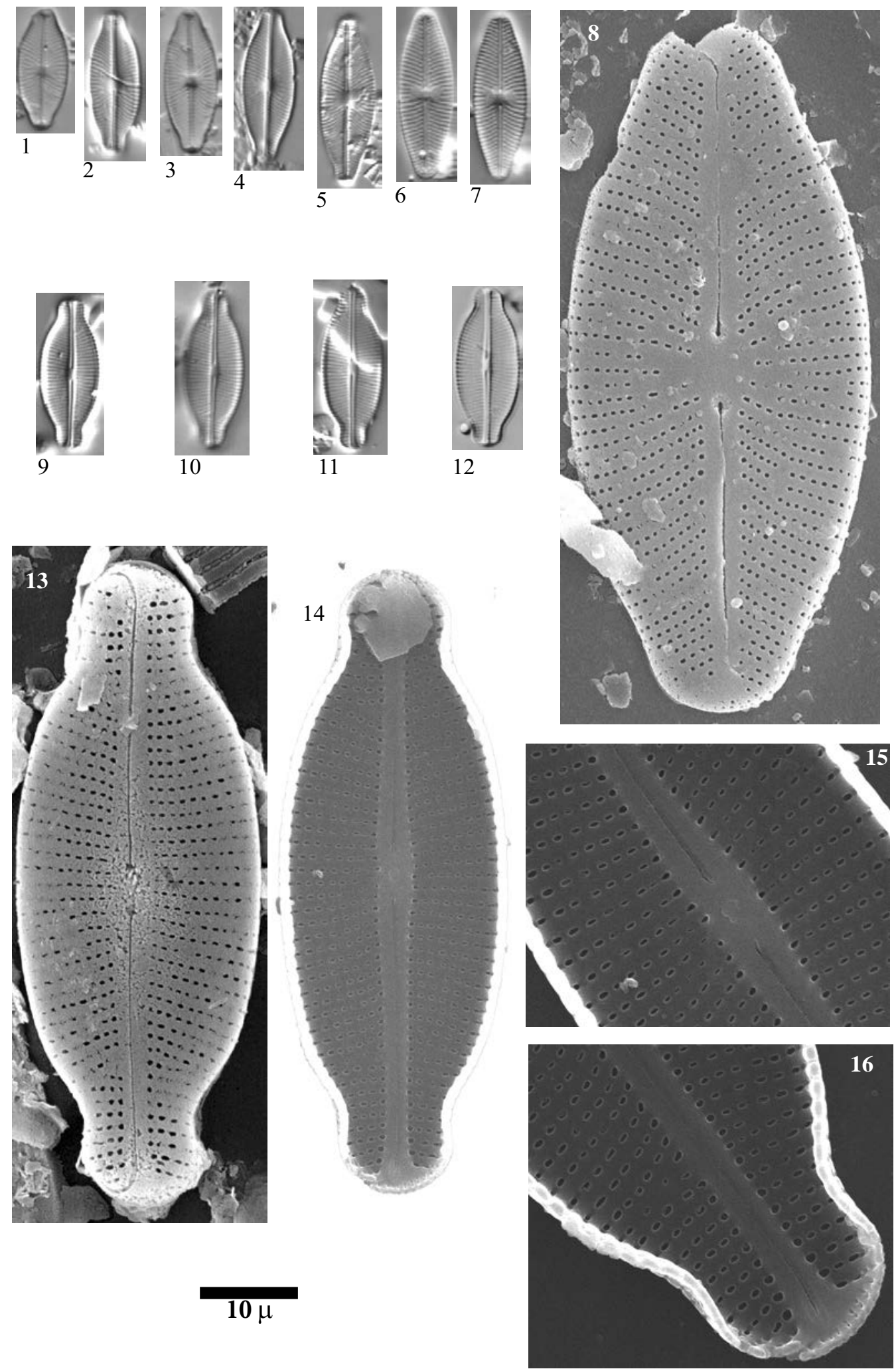

Limnetica, 36 (1): 127-395 (2017) 
Plate $66 \quad$ LM: x1500

SEM: Fig. 11 x15000, Figs. 12-13 x30000

Figs. 1-2 Geissleria cf. paludosa (Hustedt) Lange-Bertalot \& Metzeltin

Fig. 3 Geissleria sp.

Figs. 4-5 Geissleria cf. moseri Metzeltin, Witkowski \& Lange-Bertalot

Figs. 6-7, 11-13 Geissleria acceptata (Hustedt) Lange-Bertalot \& Metzeltin

Figs. 8-10 Geissleria similis (Krasske) Lange-Bertalot \& Metzeltin

Figs. 1, 2 Lake Burg, sediment BURG 1129

Fig. 3-4 Lake Posets, sediment PYR42

Fig. 5, 8-10 Lake Sen, sediment PYR40

Fig. 11-13 LLake Roumassot, sediment PYR04

Figs. 6-7 Lake Tourrat, sediment PYR23

Sample information of Plate 67

Figs. 1, 3, 26, 46

Figs. 2, 4, 45

Fig. 5

Fig. 6

Figs. 7-8, 10-11

Figs. 9, 27-28

Fig. 12

Fig. 13

Fig. 14

Figs. 15-18

Fig. 19

Fig. 20

Fig. 21

Fig. 22

Fig. 23

Fig. 24

Fig. 25

Fig. 29

Fig. 30

Lake Posets, sediment PYR42

Lake Llebreta, sediment PYR58

Lake Inf. Gallina, sediment PYR87

Lake Forcat Inf, sediment PYR77

Lake Monges, sediment EpiPYR57

Lake Burg

Lake Pondiellos, sediment PYR08

Lake Sotllo, sediment EpiPYR89

Burg, sediment BURG1093

L. Bleu de Rabassoles, sed. PYR112

Lake Plan, sediment PYR69

Lake Negre, sediment PYR79

Lake Laurenti, sediment PYR111

Lake Mariola, sediment PYR80

Lake Illa, sediment PYR66

Lake Llebreta, epilithic EpiPYR58

Lake Pica, epilithic EpiPYR100

Lake Cap Long, sediment PYR24

Lake Coronas, sediment PYR47
Fig. 31

Fig. 32

Fig. 33

Figs. 34-36, 3840,49

Fig. 37

Figs. 41-42

Figs. 43-44

Fig. 47

Fig. 48

Fig. 50

Fig. 51

Figs. 52, 59-62

Figs. 53-55

Fig. 56

Fig. 57

Fig. 58

Burg, sediment BURG 1168

Lake Chelau, epilithic EpiPYR41

Lake Estelat, sediment PYR120

L. Cregüeña, sediment PYR49

Lake Blau, sediment PYR113

Lake Sen, sediment PYR40

Lake Acherito, sediment PYR01

Lake Albe, sediment PYR96

L. Les Laquettes, sed. PYR27

Burg, sediment BURG 1062

Burg, sediment BURG 1192

Burg, sediment BURG 543

Lake Arnales, sediment PYR09

Burg, sediment BURG 853

Burg, sediment BURG 953

Burg, sediment BURG 1069 

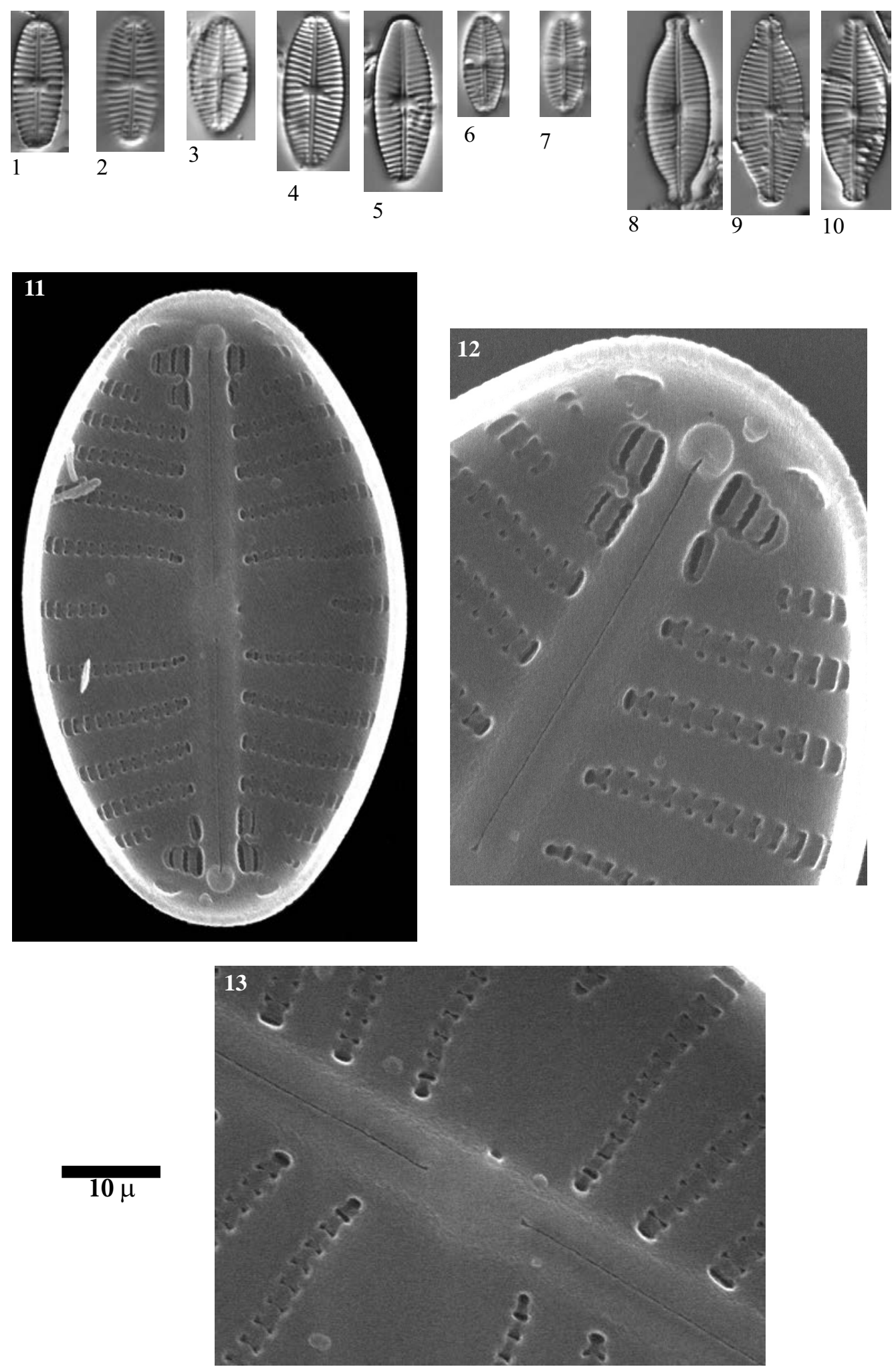

Limnetica, 36 (1): 127-395 (2017) 
Plate 67 LM: x1500, SEM: x3000

Figs. 1-5 Humidophila perpusilla (Grunow) Lowe et al.

Fig. 6 Diadesmis fukushimae Lange-Bertalot, Werum \& Broszinski

Figs. 7-9 Krasskella kriegerana (Krasske) Ross \& Sims

Figs. 10-11 Microcostatus krasskei (Hustedt) J.R. Johansen \& J.C. Sray

Fig. 12 Fallacia sp. No. 1 Pondiellos

Fig. 13 Fallacia vitrea (Østrup) Mann

Fig. $14 \quad$ Fallacia cf. insociabilis (Krasske) Mann

Figs. 15-19 Chamaepinnularia mediocris (Krasske) Lange-Bertalot

Fig. 20 Chamaepinnularia sp. No. 1 Negre

Fig. 21 Chamaepinnularia hassiaca (Krasske) Cantonati \& Lange-Bertalot

Fig. 22 Chamaepinnularia sp. No. 3 Mariola

Fig. 23 Chamaepinnularia sp. No. 2 Illa

Fig. 24 Chamaepinnularia sp 3 Julma Olkky

Fig. 25 Luticola sp. No. 1 Pica

Fig. $26 \quad$ Luticola sp. No. 2 Posets

Fig. 27 Luticola cf. nivalis (Ehrenberg) Mann

Fig. 28 Luticola sp. No. 7 Burg

Fig. 29 Luticola cf. mutica (Kützing) Mann

Fig. $30 \quad$ Luticola sp. No. 5 Coronas

Fig. $31 \quad$ Luticola sp. No. 6 Burg

Fig. 32 Luticola sp. No. 3 Chelau

Fig. 33 Luticola sp. No. 4 Estelat

Figs. 34-40 Luticola cf. goeppertiana (Bleisch in Rabenhorst) Mann

Figs. 41-42 Hippodonta costulata (Grunow) Lange-Bertalot, Metzeltin \& Witkows$\mathrm{ki}$

Figs. 43-44 Hippodonta cf. neglecta Lange-Bertalot, Metzeltin \& Witkowski

Figs. 45-48 Navicula medioconvexa Hustedt

Figs. 49 Naviculadicta multiconfusa Lange-Bertalot

Figs. 50-52 Navicula glomus Carter

Figs. 53-55 Navicula opportuna Hustedt

Figs. 56-62 Navicula pseudoventralis Hustedt sensu Krammer \& Lange-Bertalot 1986

See sample information in the previous page 

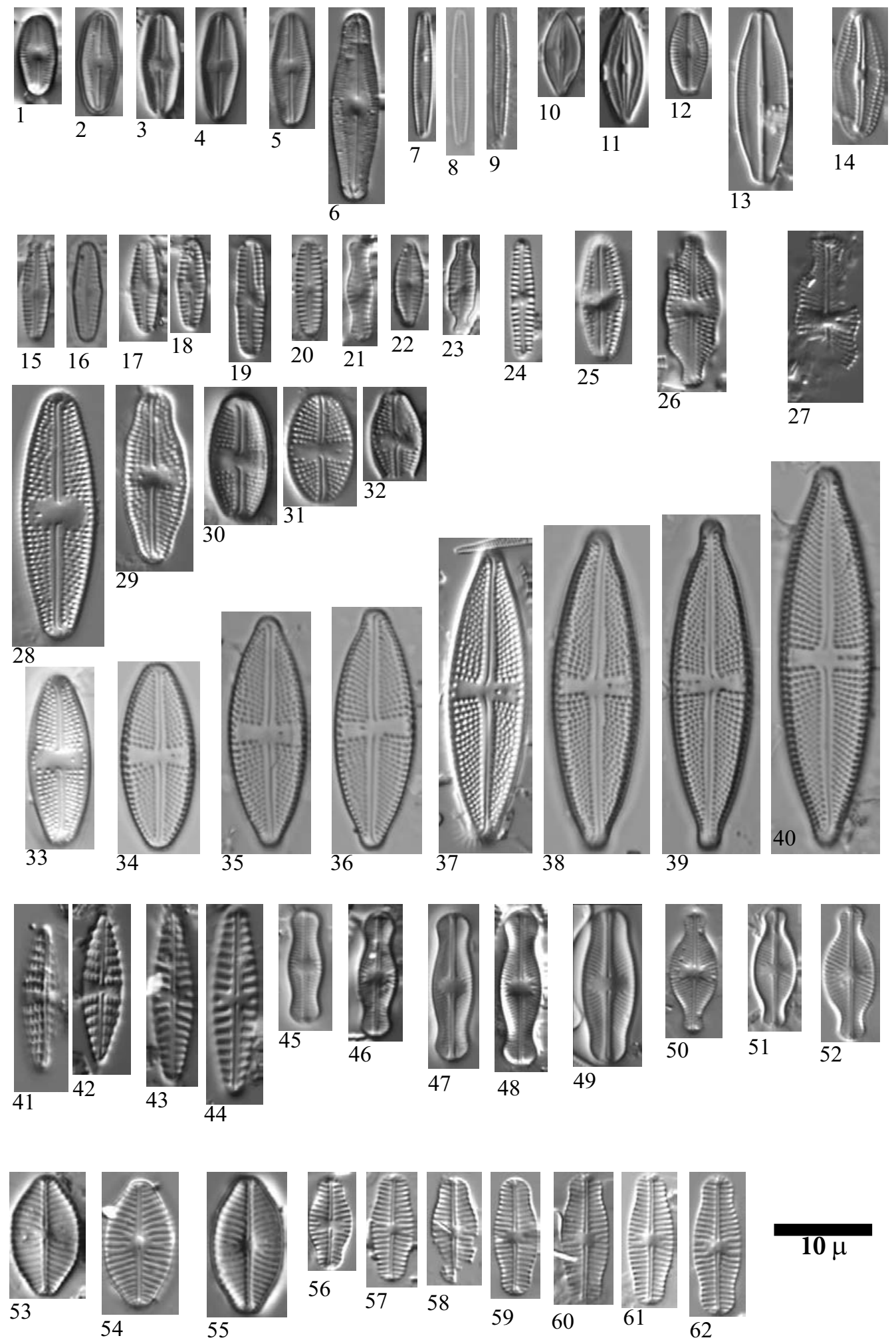
SEM: Figs. 12-13 x10000, Fig. 26, 32, 35-37 x6000

Figs. 1-6

Achnanthes carissima Lange-Bertalot

Figs. 7-13

Figs. 14-17, 26

Figs. 18-21

Figs. 22-25, 32

Figs. 27-29

Figs. 30-31

Figs. 33-37

Fig. 38

Naviculadicta sp. No. 5 Arratille Kulikovskiy
Humidophila schmassmannii (Hustedt) Buczkó et Wojtal

Genkalia sp. (Naviculadicta sp. No. 3 Arratille)

Genkalia cf. digitulus (Hustedt) Lange-Bertalot \& Kulikovskiy

Genkalia digitulus (Hustedt) Lange-Bertalot \& Kulikovskiy

aff. Navicula fluens (Naviculadicta sp. No. 4 Arratille)

Genkalia cf. digituloides (Lange - Bertalot) Lange-Bertalot \&

cf. Mayamaea atomus (Kützing) Lange-Bertalot

Fig. $1 \quad$ Lake Coronas, sediment PYR47

Figs. 2, 3-4 Lake Blaou, sediment PYR94

Figs. 5-9, 11-12, Lake Posets, sediment PYR42

18 , $20,22-25$, $33-35$

Figs. 10, 19 Lake Sen, sediment PYR40

Figs. 13, 32, Lake Redon, sediment REDOM 36-37

Figs. 14-17, 28- Lake Arratille, sediment PYR11 29, 31

Fig. 21

Lake Forcat Inf., sediment PYR77

Fig. 26 Lake Burg

Fig. 27 Lake Burg, sediment BURG 1198

Fig. 30 Lake Pondiellos, sediment PYR09

Fig. 38 Lake Burg, sediment BURG 760 

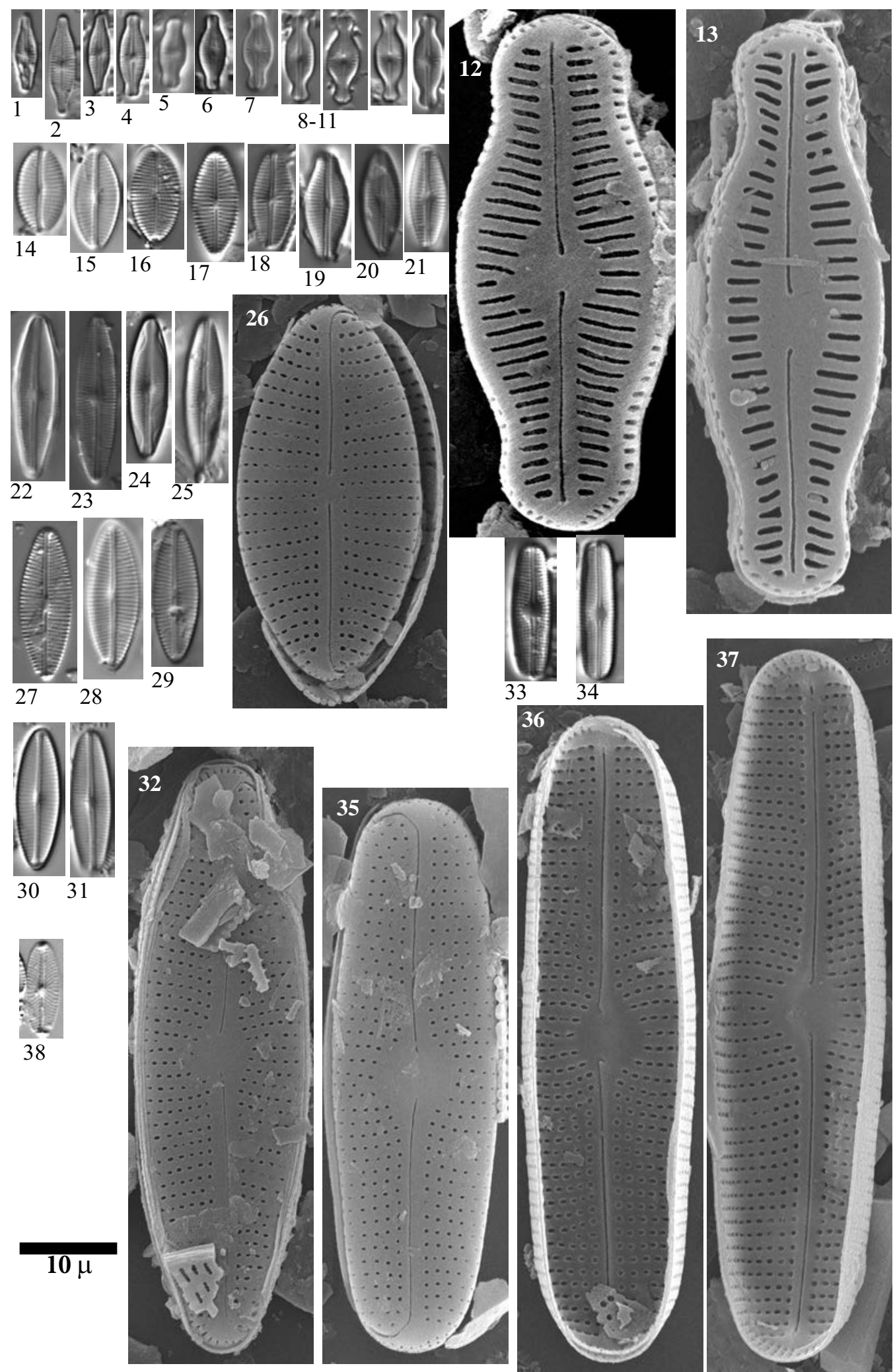

Limnetica, 36 (1): 127-395 (2017) 
Plate 69 LM: x1500

SEM: Fig. 50 x10000, Figs. 51-52 x13000

Fig. 1, $50 \quad$ Naviculadicta sp. No. 1 Ensangents

Fig. 2

Naviculadicta sp. No. 2 Bersau

Figs. 3-8

Eolimna sp. No. 5 Arnales

$15-21$

Figs. 9-14 Eolimna sp. No. 6 Marbore

Figs. 22

Eolimna spp.

Fig. 23-26 Navicula sp. No. 3 Laurenti

Fig. 27

Navicula sp. No. 4 Laquettes

Figs. 29-34

Sellaphora cf. seminulum (Grunow) D.G. Mann

Figs. 35-40 Sellaphora cf. seminulum (Grunow) D.G. Mann

Figs. 41-43 Navicula utermoehli Hustedt

Fig. 44

Navicula cf. submuralis Hustedt

Figs. 45-49

Navicula sp. No. 7 Bergus

Figs. 51-52 Navicula spp.

\begin{tabular}{|c|c|c|c|}
\hline Figs. 1, 5 & Lake Ensangents, sediment PYR106 & Fig. 33,35 & L. Inf. de la Gallina, sed. PYR87 \\
\hline Fig. 2 & Lake Bersau, epilithic EpiPYR03 & Fig. 34 & PYR127 \\
\hline Figs. 3-4, 45 & Lake Port Bielh, sediment PYR28 & Fig. 37 & L. Burg, sediment BURG 848 \\
\hline Figs. 6-7, 14, 20, 49 & Lake Siscar, sediment PYR126 & Fig. 38 & L. Burg, sediment BURG 851 \\
\hline Fig. 8 & L. Burg, sediment BURG 932 & Fig. 39 & L. Burg, sediment BURG 932 \\
\hline Figs. 9, 11 & L. Helado de Marboré, sed. PYR18 & Fig. 40 & L. Burg, sediment BURG 853 \\
\hline $\begin{array}{l}\text { Figs. 10-13,18, } \\
23,26\end{array}$ & L. Burg & Fig. 41 & L. Burg, sediment BURG 831 \\
\hline Figs. 12, 27 & Lake Laurenti, sediment PYR111 & Fig. $42-44$ & Lake Arratille, sediment PYR11 \\
\hline Fig. 15 & Lake Arnales, sediment PYR09 & Fig. 46 & L. Burg, sediment BURG 698 \\
\hline Fig. 16 & L. Burg, sediment BURG 906 & Fig. $47-48$ & L. Gelat Bergús, sediment PYR65 \\
\hline Fig. 17 & L. Burg, sediment BURG 1007 & & \\
\hline Figs. 19, 25, 28 & $\begin{array}{l}\text { Lake Les Laquettes, sediment } \\
\text { PYR } 27\end{array}$ & & \\
\hline Fig. 22 & L. Burg, sediment BURG 837 & & \\
\hline Fig. 24 & L. Burg, sediment BURG 1153 & & \\
\hline Fig. 26 & L. Burg, sediment BURG 1053 & & \\
\hline Fig. 32 & Lake Sen, sediment PYR40 & & \\
\hline
\end{tabular}



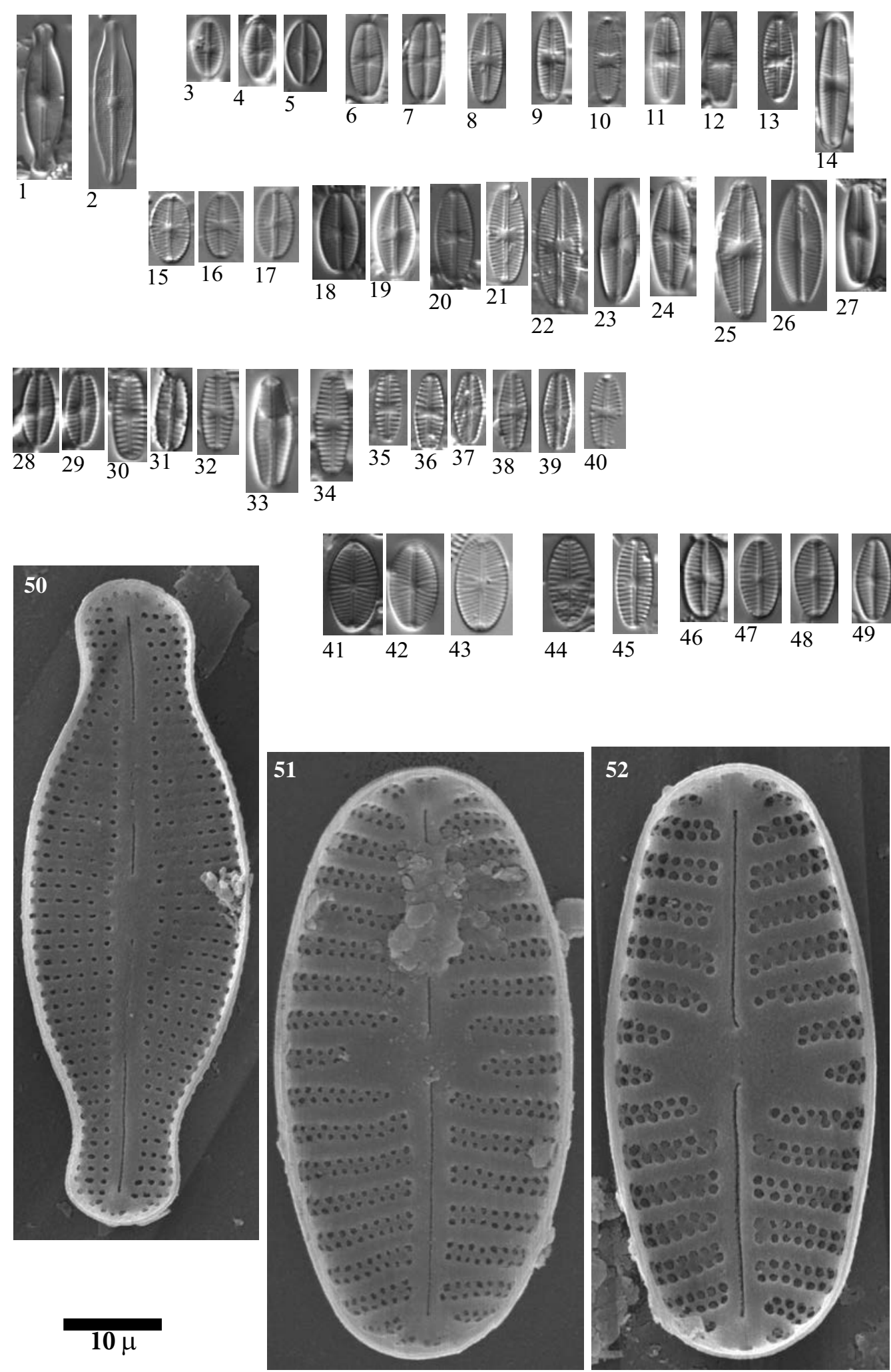

Limnetica, 36 (1): 127-395 (2017) 
Fig. 1 Adlafia cf. suchlandtii (Hustedt) Lange-Bertalot

Fig. 2 Adlafia bryophila (Petersen) Lange-Bertalot

Figs. 3-4 Adlafia aquaeductae (Krasske) Moser, Lange-Bertalot \& Metzeltin

Fig. 5 Adlafia sp. No. 1 Barroude

Fig. $6 \quad$ Kobayasiella parasubtilissima (Kobayasi \& Nagumo) Lange-Bertalot

Figs. 7-8 Kobayasiella subtilissima (Cleve) Lange-Bertalot

Figs. 9-11 Navicula brockmanni Hustedt

Fig. 12 Adlafia bryophila (Petersen) Lange-Bertalot sensu lato

Fig. 13 Adlafia cf. minuscula (Grunow) H. Lange-Bertalot

Figs. 14-16 Adlafia minuscula (Grunow) H. Lange-Bertalot

Figs. 17-19 Adlafia cf. suchlandtii (Hustedt) Lange-Bertalot

Fig. 20 Sellaphora cf. nanoides Lange-Bertalot, Cavacini, Tagliaventi \& Alfinito

Fig. $21 \quad$ Navicula sp. No. 1 Laurenti

Figs. 22, 25 Naviculadicta cf. difficillima Hustedt

Figs. 23-24 Naviculadicta cf. stauroneioides Lange-Bertalot

Figs. 26-29 Navicula absoluta Hustedt sensu lato

Fig. 30 ? Placoneis sp

Fig. $31 \quad$ cf. Navicula gerloffii Schimanski

Figs. 32-38 Navicula laterostrata Hustedt

Figs. 39-40 Kobayasiella sp. 1 Seno cf. Nupela tenuicephala (Hustedt) Lange-Bertalot

Fig. $41 \quad$ Kobayasiella sp. 2 Bleu

Fig. 1 Lake Coronas, sediment PYR70

Fig. 2 Lake Blaou, epilithic EpiPYR43

Fig. 3 Lake Posets, sediment PYR01

Fig. 4 Lake Sen, sediment PYR43

Fig. 5 Lake Blaou, epilithic EpiPYR29

Figs. 6, 40 Lake Sen, sediment PYR57

Fig. 7 Lake Sen, sediment PYR85

Fig. 8 Lake Sen, sediment PYR84

See next page for the others samples 

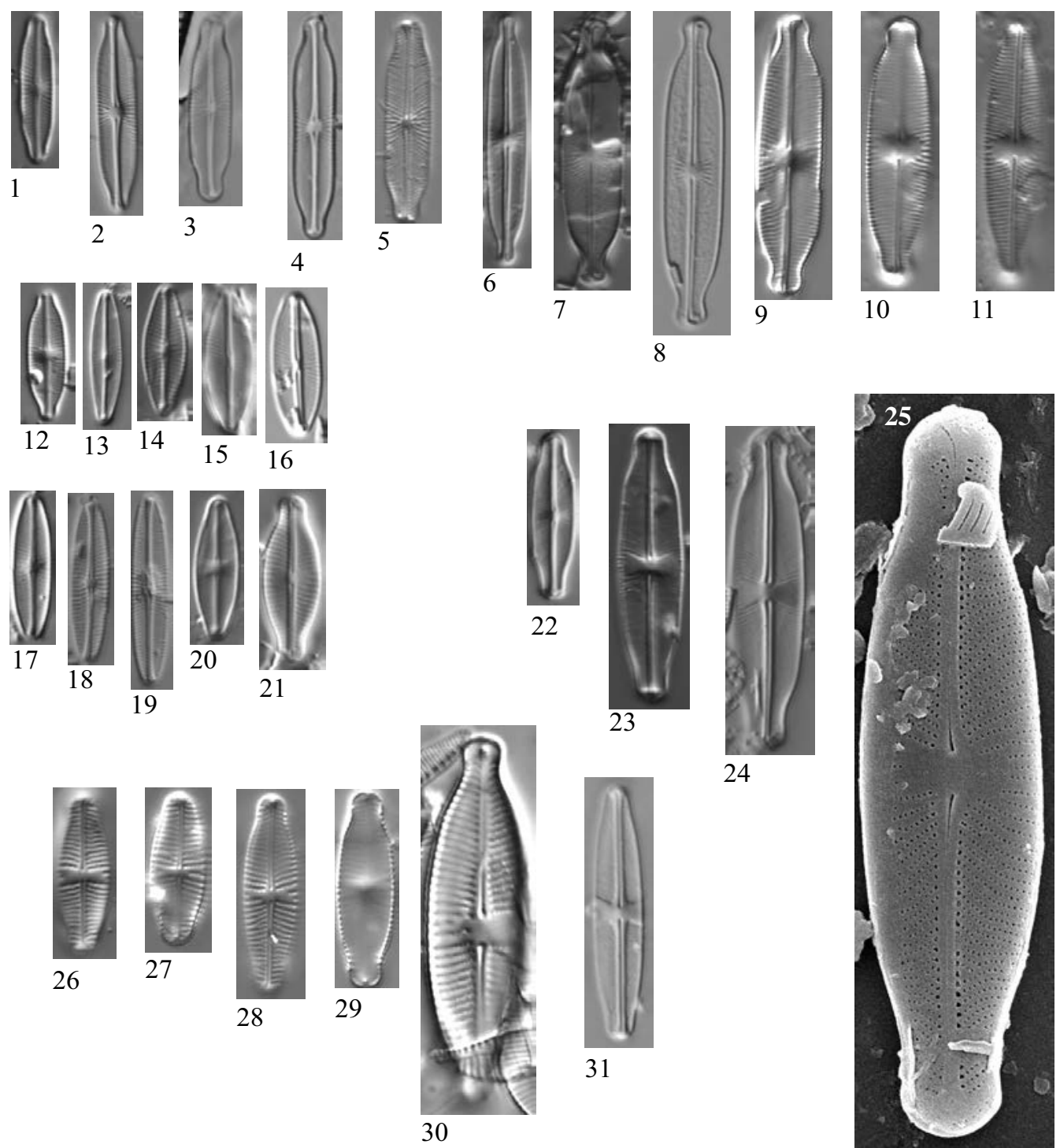

24

30
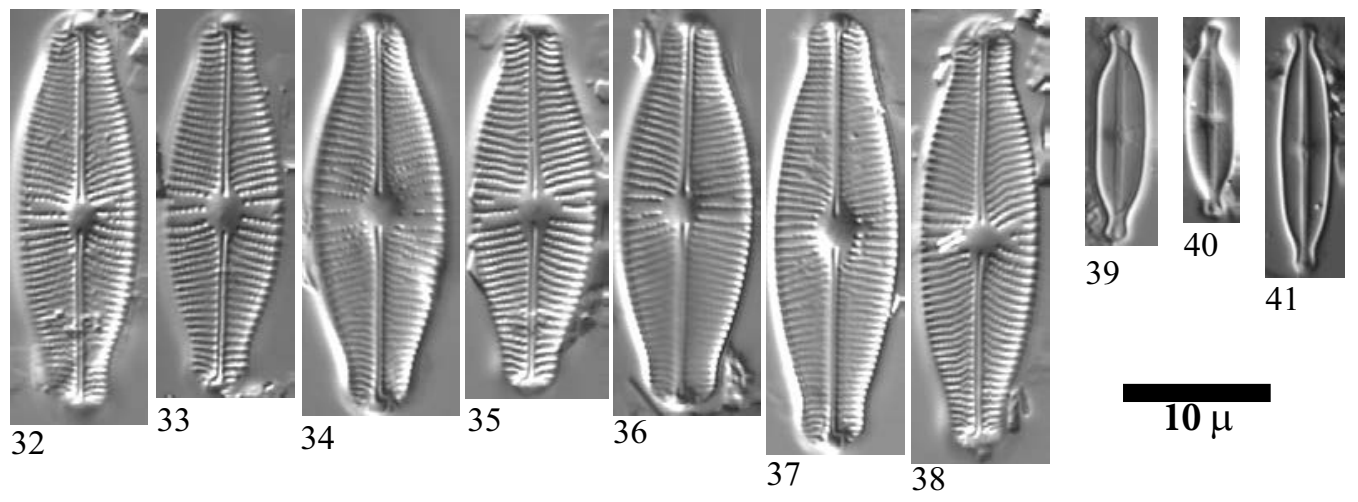
Plate $71 \quad$ LM: $x 1500$

SEM: $\mathrm{x} 4500$

Figs. 1-6 Naviculadicta vitabunda (Hustedt) Lange-Bertalot

Fig. $7 \quad$ Craticula molestiformis (Hustedt) Mayama

Figs. 8-9 Craticula submolesta (Hustedt) Lange-Bertalot

Fig. $10 \quad$ Craticula cf. vixnegligenda Lange-Bertalot

Fig. $11 \quad$ Craticula sp. No. 1 Burg

Fig. 12 Craticula cuspidata (Kützing) Mann

Fig. 1 Lake Burg, sediment BURG 760

Figs. 2-3 Lake Port Bielh, sediment PYR28

Fig. $4 \quad$ Lake Canals Roges, sediment PYR124

Figs. 5-6 Lake Burg, sediment BURG 543

Fig. 7 Lake Siscar, sediment PYR126

Fig. 8 Lake Coronas, sediment PYR47

Fig. 9 Lake Redon, sediment REDOM

Fig. $10 \quad$ Lake Llosás, sediment PYR46

Fig. 11 L. Burg, sediment BURG 1070

Sample information of Plate 70

Fig. 9 L. Burg, sediment BURG 880

Lake Burg, sediment BURG 987

Fig. 35 Lake Burg, sediment BURG 694

Figs. 10-11

$\begin{array}{ll}\text { Figs. } 12 & \text { Lake Mariola, epilithic EpiPYR80 } \\ \text { Fig. } 13 & \text { Lake Arratille, sediment PYR11 }\end{array}$

Figs. LLake Senó, epilithic EpiPYR84 39

Fig. 41 L. Bleu de Rabassoles, EpiPYR112

Figs. 14, 20

Lake Cap Long, sediment PYR24

Figs. 15, 17

Lake Siscar, sediment PYR126

Figs. 16

Lake Bleu, epilithic EpiPYR22

Figs. 18-19

Lake Inf. de la Gallina, sediment PYR87

Fig. 21

Lake Laurenti, sediment PYR111

Figs. 22-23

Lake Coronas, sediment PYR47

Fig. 24

Lake Albe, sediment PYR96

Fig. 26

Lake Negre, epilithic EpiPYR108

Fig. 27

Lake Burg, sediment BURG 1080

Fig. 28-29

Lake Burg, sediment BURG 543

Fig. 30

L. Helado Monte Perdido, sediment PYR19

Fig. 31

Lake Mariola, sediment PYR80

Figs. 32-34, 36-38

Lake Burg, sediment BURG 543 

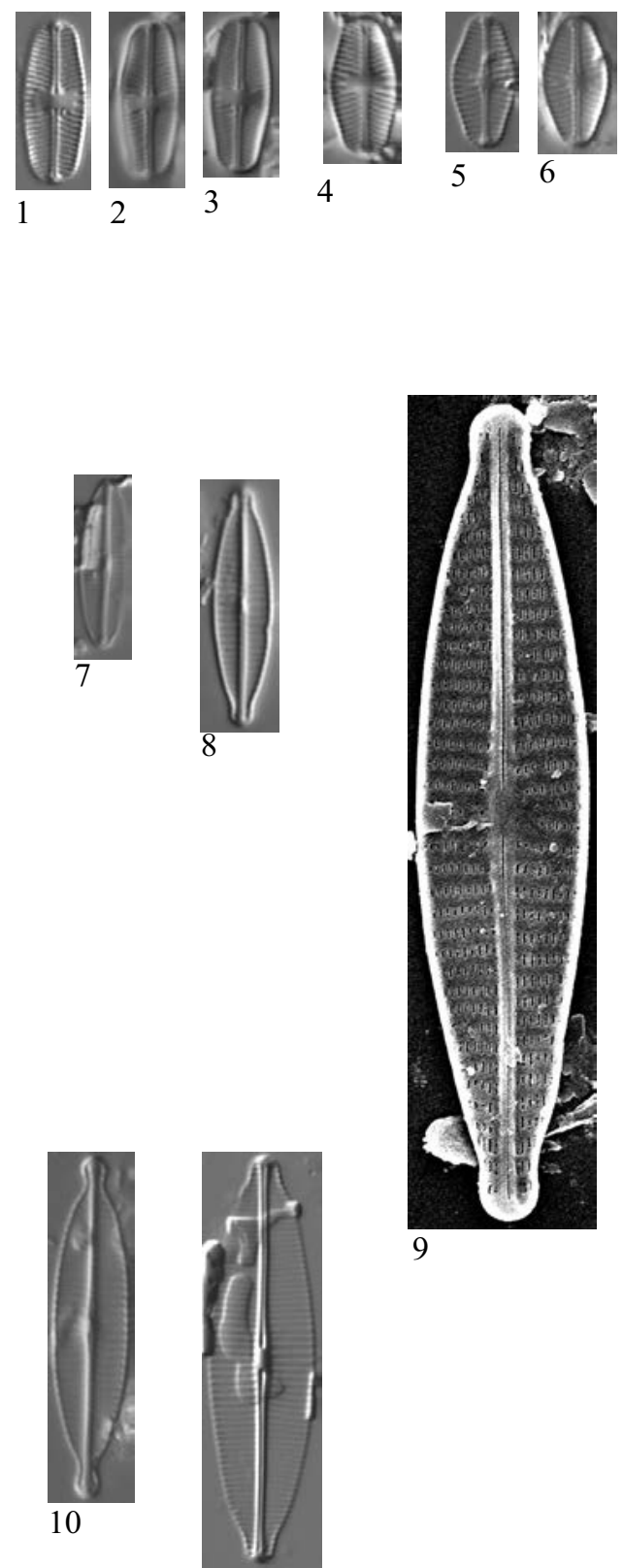

11
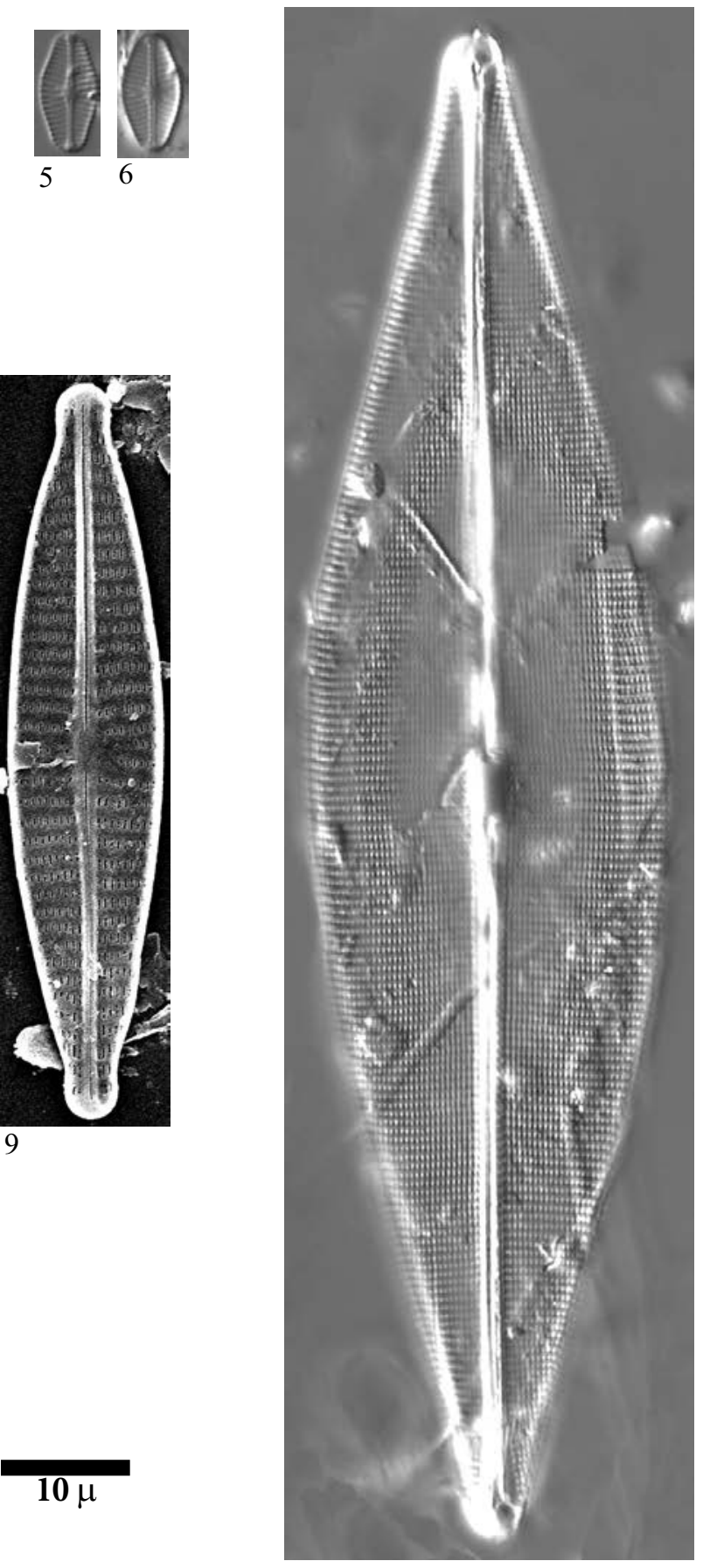

12 
Plate $72 \quad$ LM: $x 1500$

SEM: Fig. 4 x1100, Fig. 5 x4000, Fig. 11 x10000

Fig. 1 Gyrosigma sp. No. 2 Mora

Figs. 2-9 Gyrosigma sp. No. 1 Sen

Fig. 1 Lake Basa de la Mora, sediment PYR32

Figs. 2-3 Lake Sen, sediment PYR40

Figs. 6-7 Lake Arratille, sediment PYR11

Figs. 4-5, 9 Lake Laurenti, sediment PYR111 

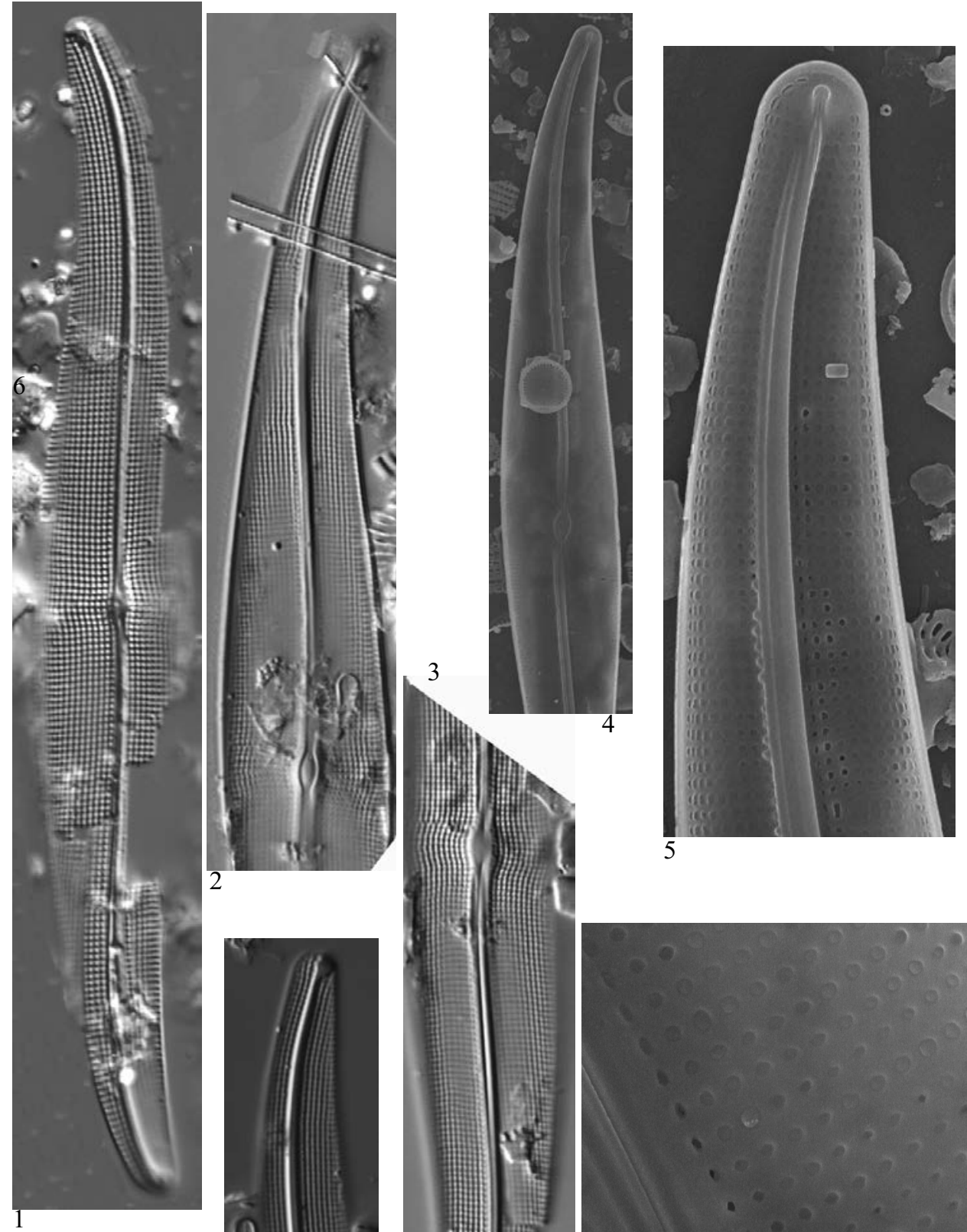

2
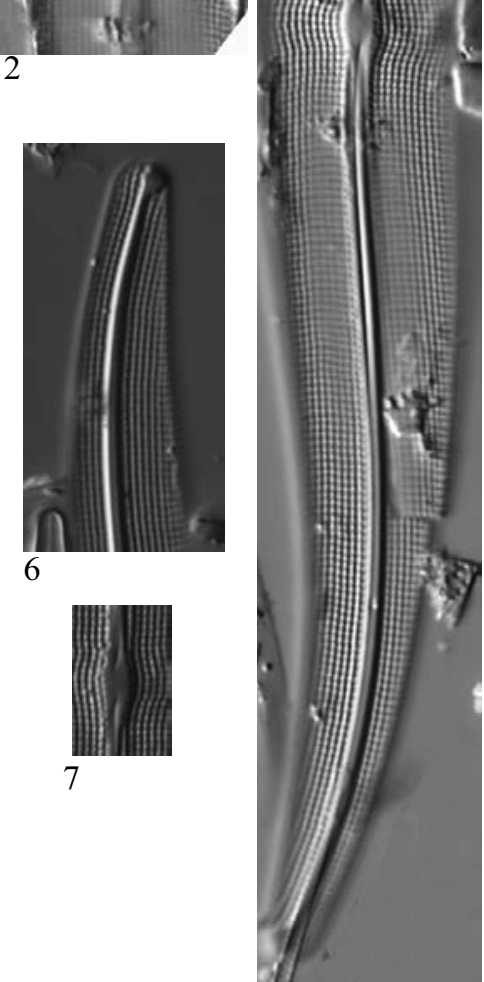

9

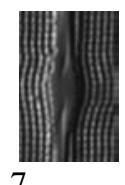

7

$10 \mu$

Limnetica, 36 (1): 127-395 (2017) 
Plate $73 \quad$ LM: $x 1500$

SEM: Fig. 9 x5000, Fig. $17 \times 2500$

Figs. 1-9 Neidium alpinum Hustedt

Figs. 10-13 Neidium affine (Ehrenberg) Pfitzer sensu lato

Figs. 14-15 Neidium longiceps (Gregory) Ross

Fig. $16 \quad$ Neidiopsis cf. levanderi (Hustedt) Lange-Bertalot \& Metzeltin

Fig. 17 Neidium sp.

Fig. $18 \quad$ Neidium cf. dubium (Ehrenberg) Cleve

Fig. 19-20 Neidium sp. No. 1 Illa

Figs. 1, 6, 15 Lake Gelat Bergús, sediment PYR65

Fig. 2

Lake Monges, sediment PYR57

Figs. 3, 5, 19 Lake Illa, sediment PYR66

Fig. $4 \quad$ Lake Angonella, sediment PYR78

Fig. 7 Lake Negre, sediment PYR79

Fig. 8 Lake Bleu de Rabassoles, sediment PYR112

Fig. 9 Lake Bersau, sediment PYR03

Fig. 10 Lake Sen, sediment PYR40

Fig. 11 Lake Posets, sediment PYR42

Fig. 12 Lake Aixeus, sediment PYR92

Fig. 13 Lake Forcat Inf., sediment PYR77

Fig. 14 Lake Bachimala, sediment PYR31

Fig. 16 Lake Port Bielh, sediment PYR28

Fig. 17 Lake Arnales, epilithic EpiPYR09

Fig. 18 Lake Acherito, sediment PYR01

Fig. $20 \quad$ Lake Senó, sediment PYR84

Fig. $9 \quad$ Manfred Ruppel photo 

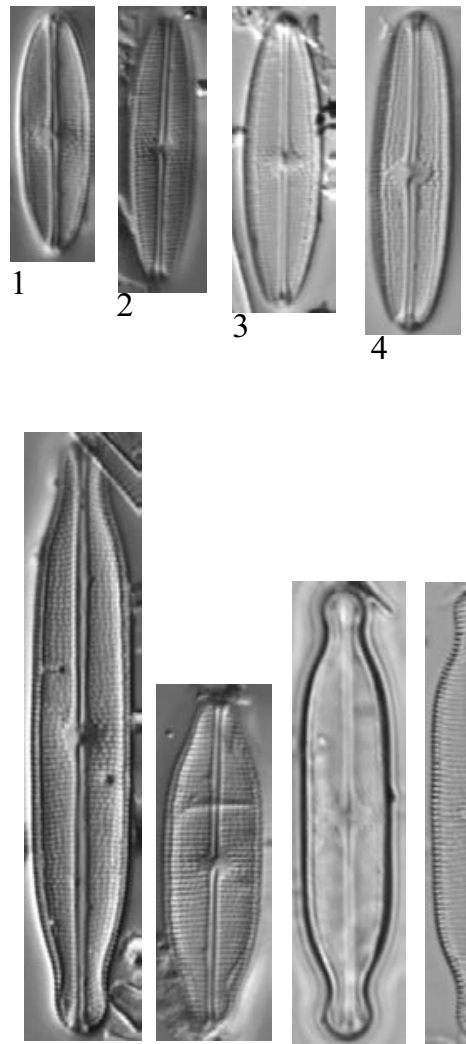

10
11

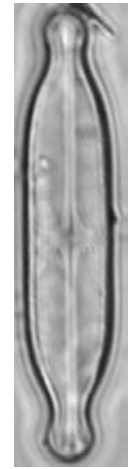

12

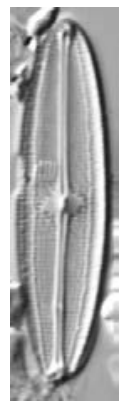

5
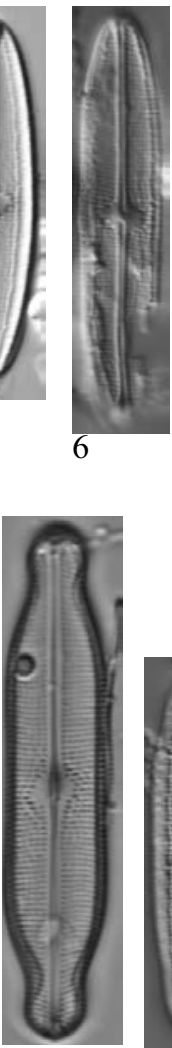

14
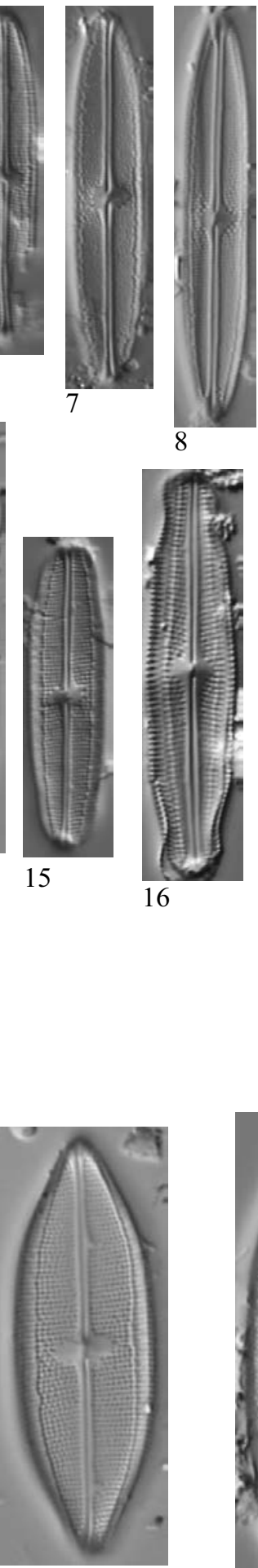

19

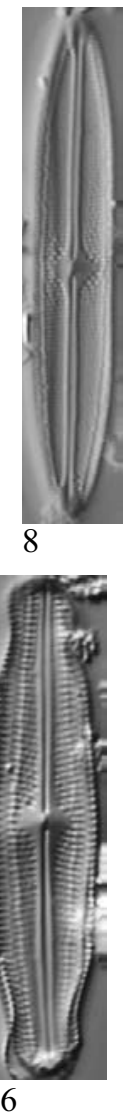

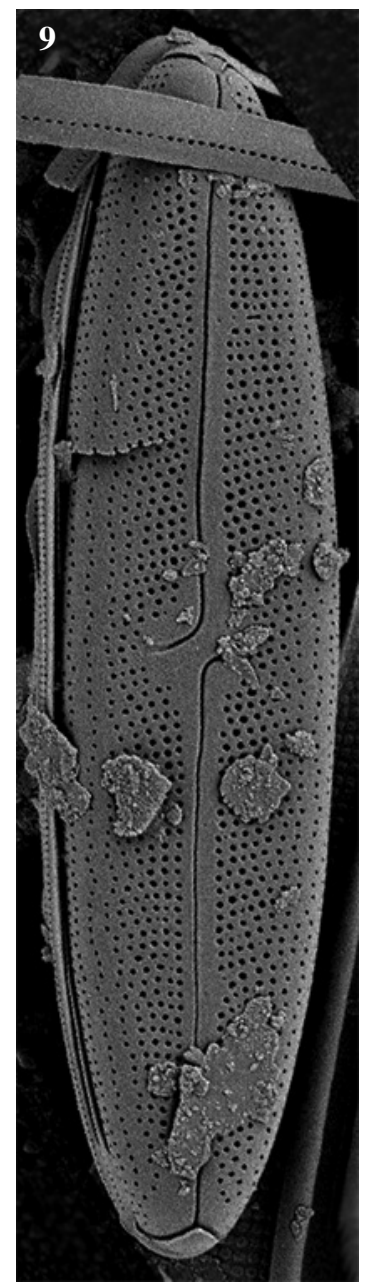

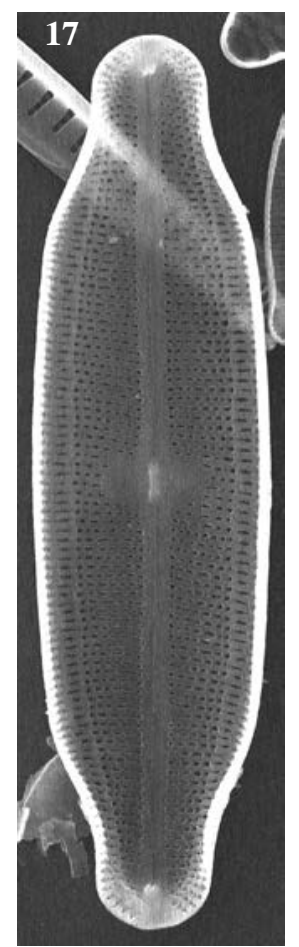

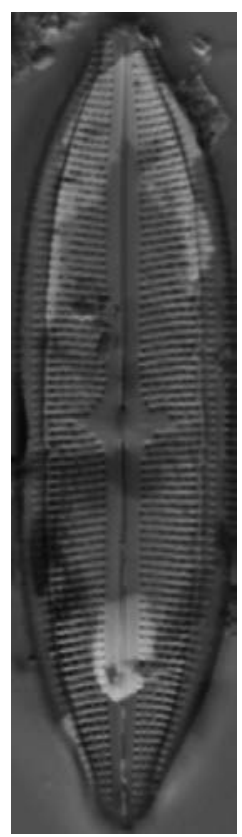

18

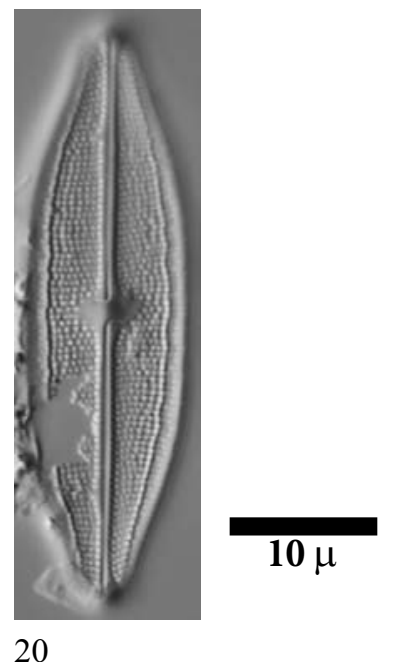

Limnetica, 36 (1): 127-395 (2017) 
Plate $74 \quad$ LM: $x 1500$

SEM: $x 3000$

Figs. 1-2

Neidium sp. No. 2 Illa

cf. Julma 1 in Lange-Bertalot \& Metzeltin 1996

Fig. 3

Neidium sp. No. 3

cf. Julma 5 in Lange-Bertalot \& Metzeltin 1996

Fig. 4

Neidium cf. ampliatum (Ehrenberg) Krammer

Fig. 5

Neidium sp. No. 4

cf. Julma 2 in Lange-Bertalot \& Metzeltin 1996

Fig. 6

Neidium bisulcatum (Lagerstedt) Cleve sensu Krammer

Figs. 1, 3, 5 Lake Illa, sediment PYR66

Fig. 2

Lake Garbet, sediment PYR81

Fig. 4

Lake Arratille, sediment PYR11

Fig. 6

Lake Port Bielh, sediment PYR28 

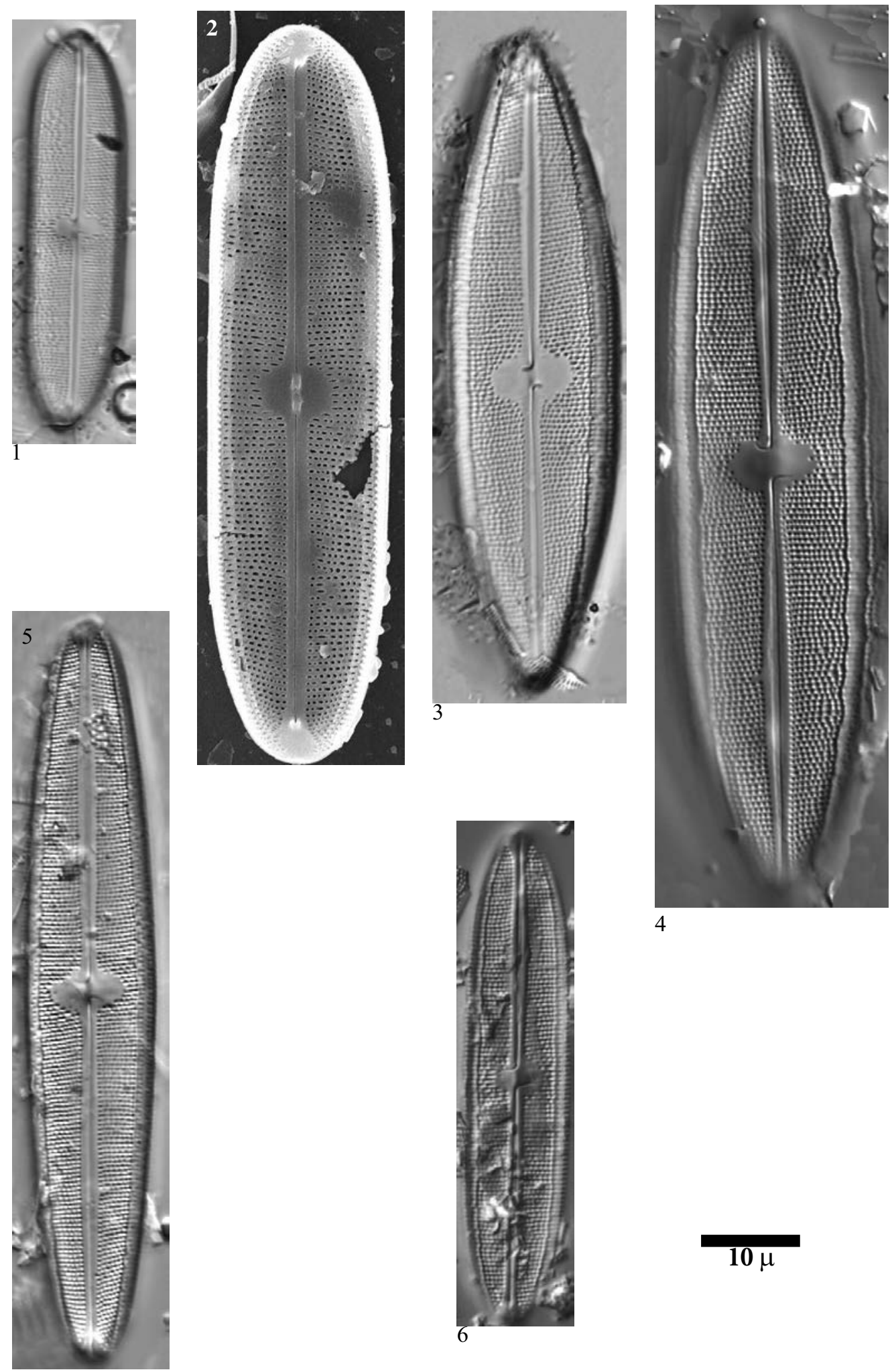

4

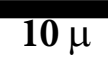

Limnetica, 36 (1): 127-395 (2017) 
Plate $75 \quad$ LM: $x 1500$

SEM: $\times 2500$

Figs. 1, 8

Stauroneis sp. No. 8 Illa

Stauroneis cf. acidoclinata Lange-Bertalot \& Werum

Figs. 2-7

Stauroneis cf. acidoclinata Lange-Bertalot \& Werum

Figs. 9-10

Stauroneis cf. reichardtii Lange-Bertalot, Cavacini, Tagliaventi \& Alfinito

Figs. 11-13 Stauroneis smithii Grunow

Figs. 14-18 Stauroneis neohyalina Lange-Bertalot \& Kramme

Figs. 19-21 Stauroneis sp. No. 9 Forcat

Figs. 1, 3 Lake Illa, sediment PYR66

Figs. 2, 4, $6 \quad$ Lake Posets, sediment PYR42

Figs. 5, 7, 18- Lake Forcat Inf., sediment PYR77

19

Fig. 8 Lake Baiao Superior, sediment PYR76

Figs. 9-12 Palaeolake Burg

Fig. 13 Lake Helado de Marboré, sediment PYR18

Figs. 14-16 Lake Inf. de la Gallina, sediment PYR87

Fig. 17 Lake Pixón, sediment PYR44

Figs. 20-21 Lake Redon, sediment REDOM 

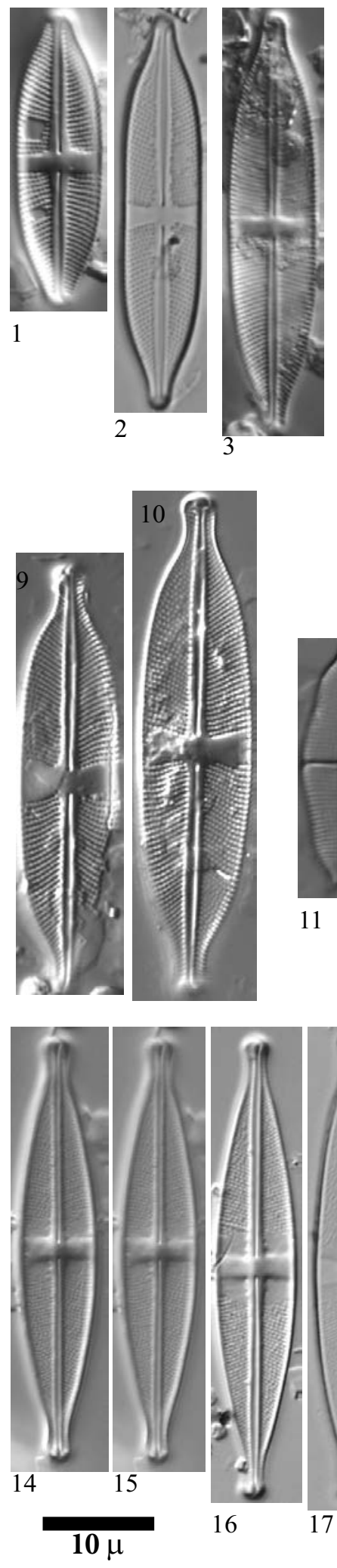

11
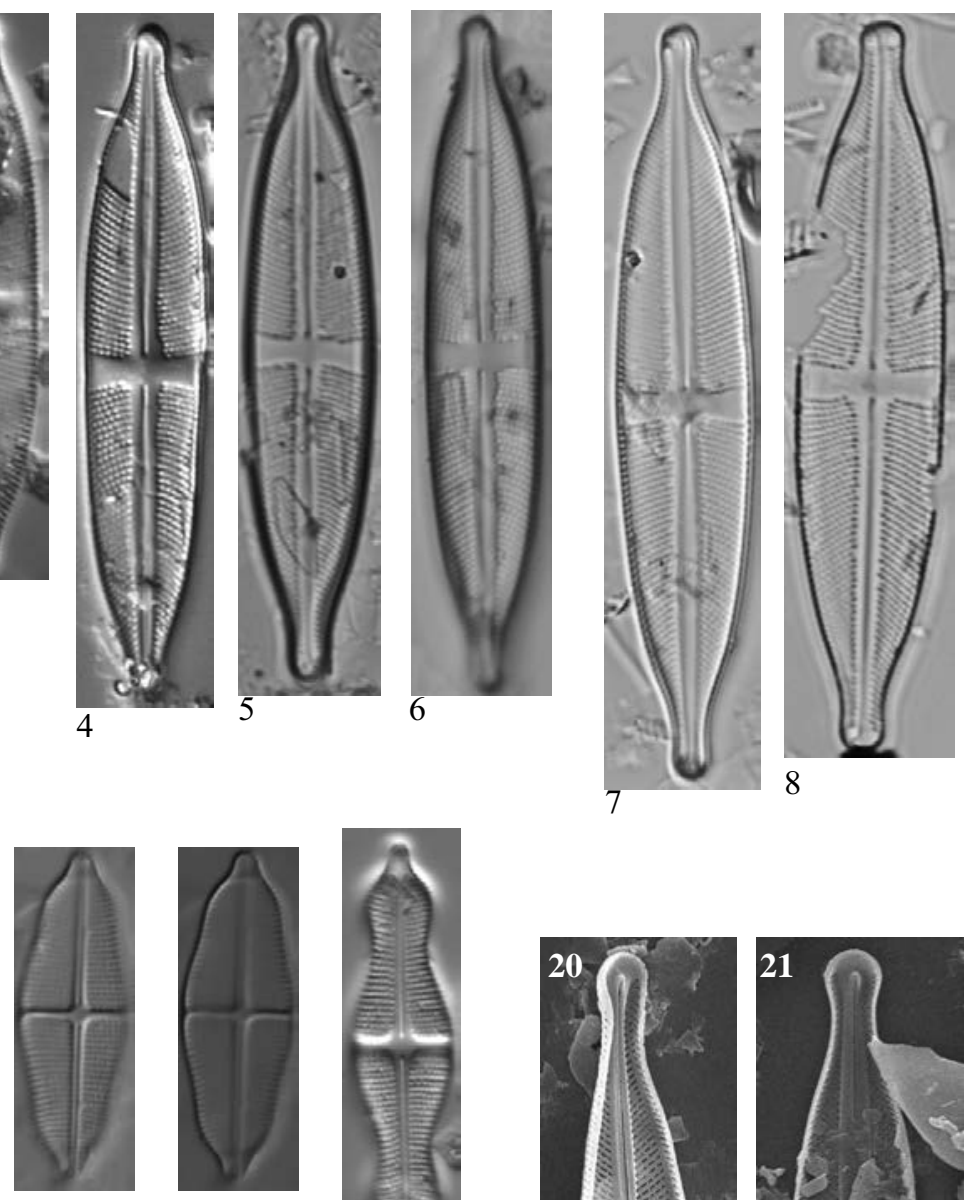

12
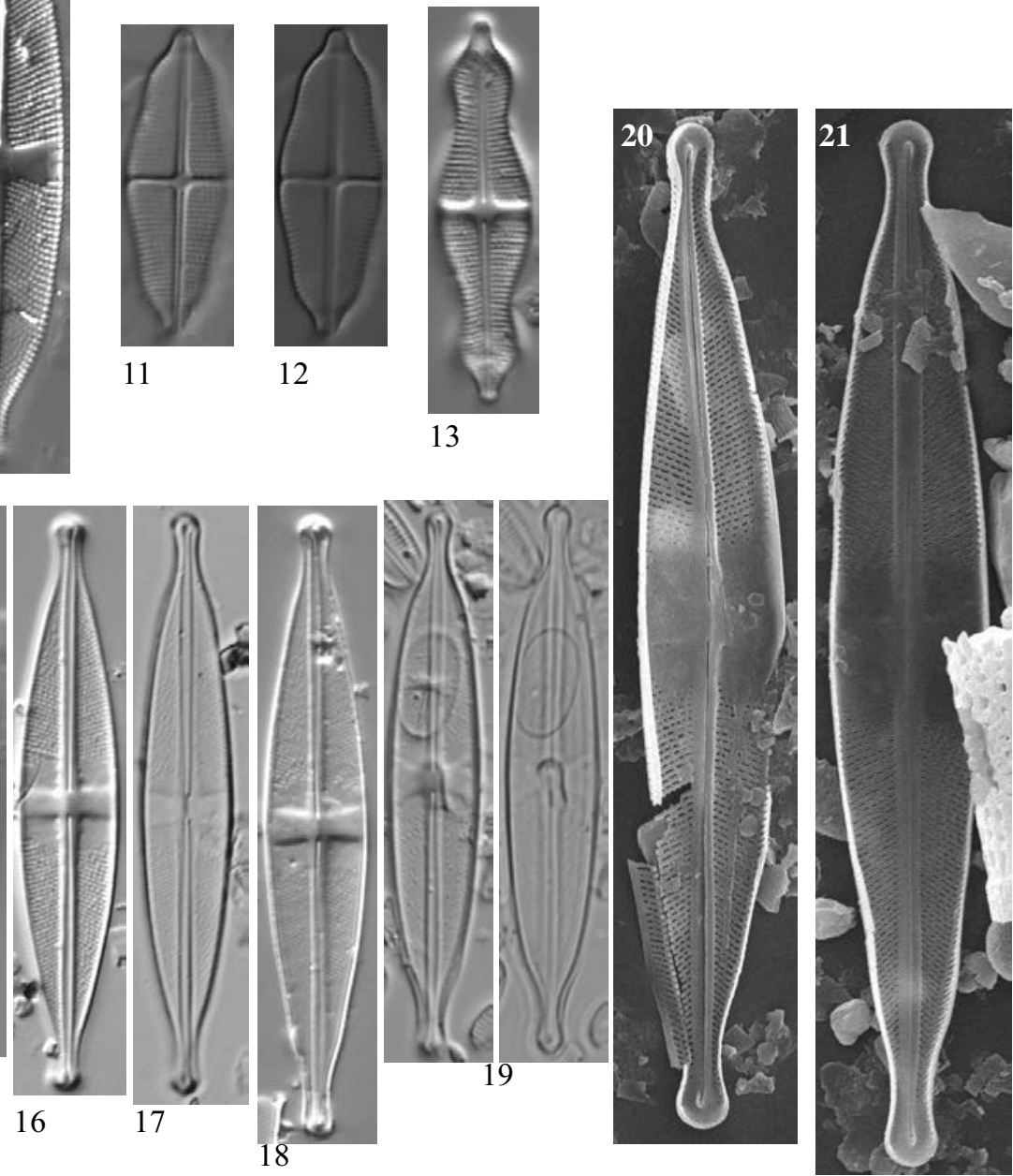

Limnetica, 36 (1): 127-395 (2017) 
Plate $76 \quad$ LM: $x 1500$

Fig. 1

Figs. 2-3

Fig. 4

Figs. 5-6

Figs. 7-8

Figs. 9-10

Fig. 11

Figs. 12-13

Fig. 14

Fig. 1

Figs. 2, 11

Fig. 3

Fig. 4

Fig. 5

Fig. 6

Fig. 7

Fig. 8

Fig. 9

Fig. 10

Figs. 12-13

Fig. 14
Stauroneis sp. No. 1 Tristaina

Stauroneis sp. aff. borrichii Lund, No. 2 Illa

Stauroneis sp. No. 3 Negre

Stauroneis siberica (Grunow) Lange-Bertalot \& Krammer

Stauroneis sp. No. 4 Burg

Stauroneis gracilis Ehrenberg

Stauroneis sp. No. 5 Illa

Stauroneis sp. No. 6 Burg

Stauroneis sp. aff. borrichii Lund, No. 7 Burg
Lake Mes Amunt de Tristaina, sediment PYR86

Lake Illa, sediment PYR66

Lake Cregüeña, sediment PYR49

Lake Negre, sediment PYR79

Lake Arratille, sediment PYR11

Lake Port Bielh, sediment PYR28

Lake Burg, sediment BURG 616

Lake Burg, sediment BURG 729

Lake Posets, sediment PYR42

Lake Les Laquettes, sediment PYR27

Lake Burg, sediment BURG 755

Lake Burg 

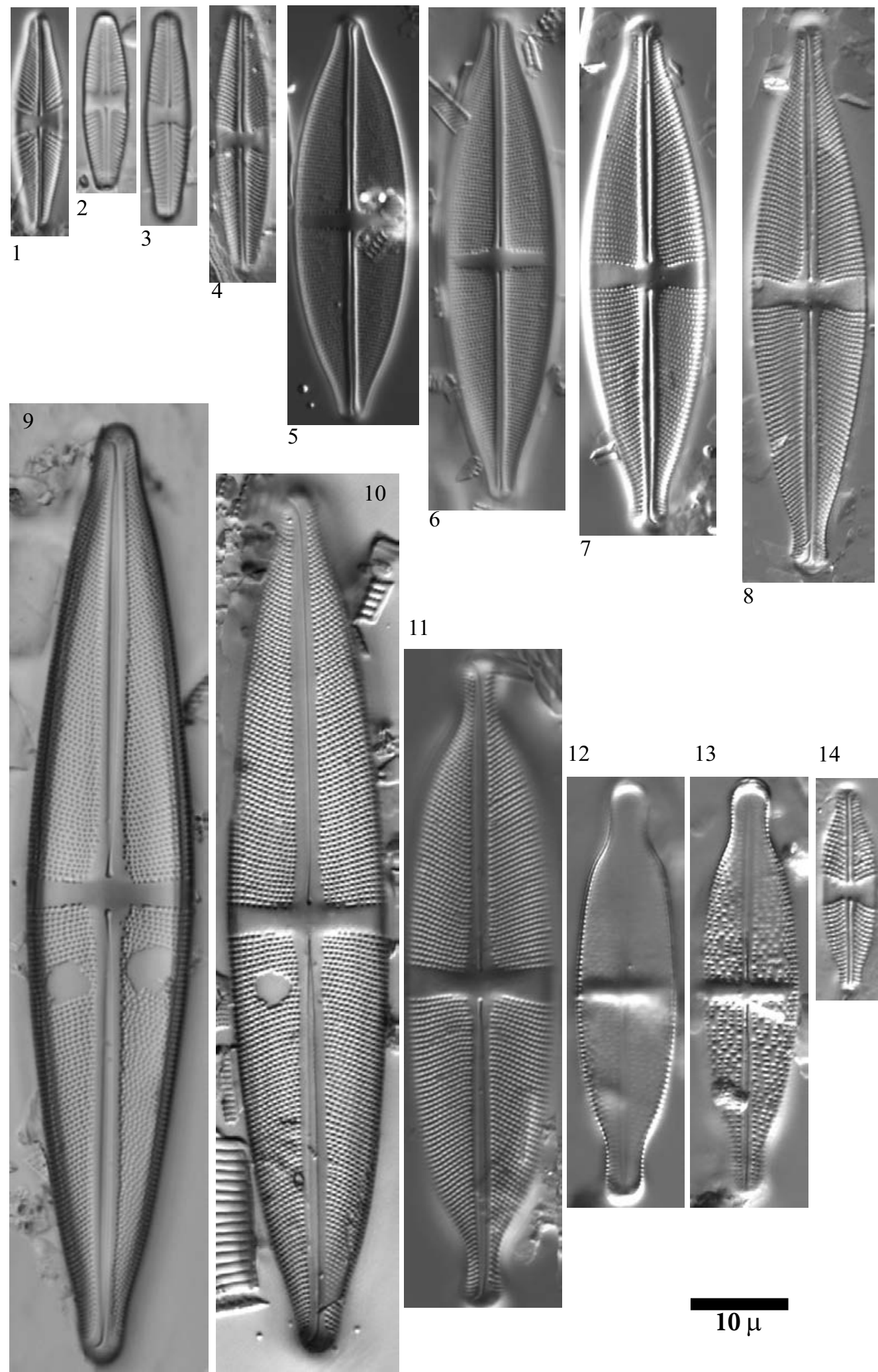

11
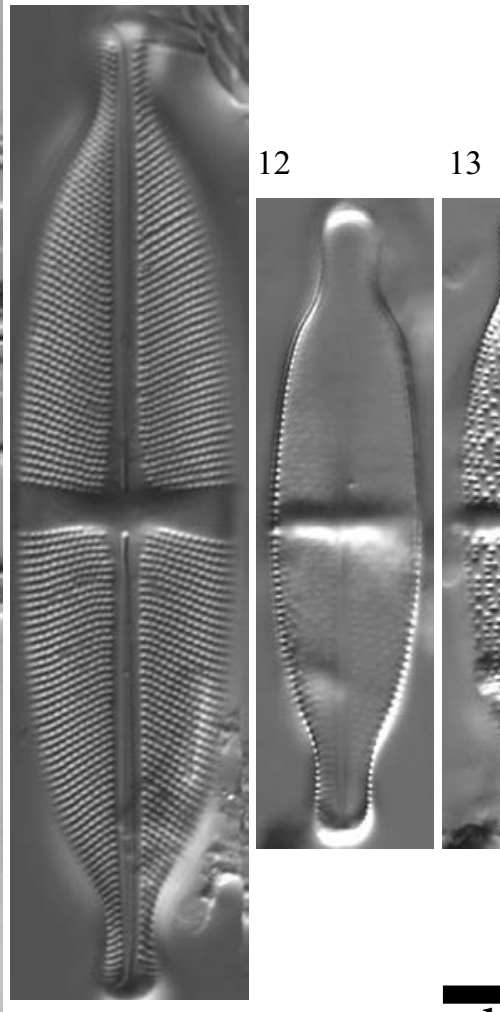

$$
\text { है }
$$
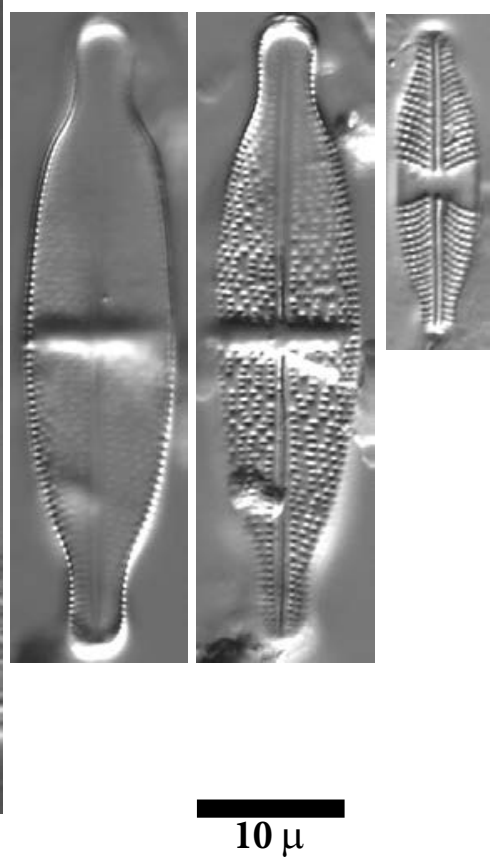

Limnetica, 36 (1): 127-395 (2017) 
Plate $77 \quad$ LM: $x 1500$

SEM: $11-14 \times 4000,15 \times 25000$

Fig. 1 Brachysira zellensis (Grunow) Round \& Mann

Figs. 2-6, $10 \quad$ Brachysira brebissonii Ross

Figs. 7-8 Brachysira intermedia (Østrup) Lange-Bertalot 11-13

Fig. 9

Brachysira cf. brebissonii Ross

Fig. 1 Lake Arratille, sediment PYR11

Fig. 2 Lake Baiao Superior, sediment PYR76

Figs. 3, 7-9 Lake Seno, sediment PYR84

Fig. 4 Lake Aixeus, sediment PYR92

Figs. 5-6, 13 Lake Posets, sediment PYR42

Figs. 10-11 Lake Redon, sediment REDOM

Fig. 12 Lake Port Bielh, epilithic PYR28 

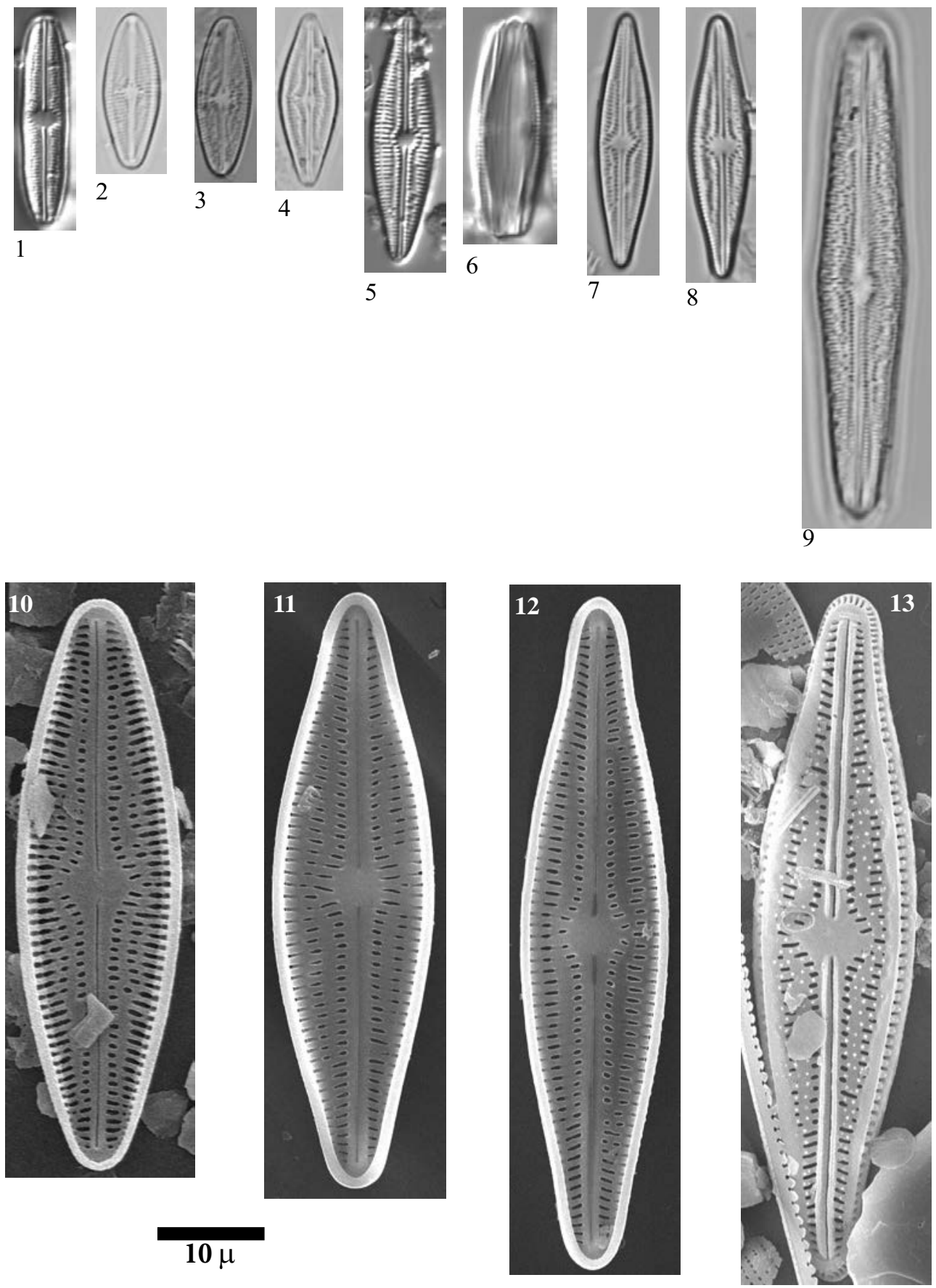
Figs. 1-12, 15-16 Brachysira neoexilis Lange-Bertalot $18-22$

Figs. 13-14

Brachysira cf. procera Lange-Bertalot

Fig. 17

Brachysira cf. neglectissima Lange-Bertalot

Figs. 1, 10, $12 \quad$ Lake Les Laquettes, sediment PYR27

Figs. 2-3, $15 \quad$ Lake Posets, sediment PYR42

Fig. $4 \quad$ Lake Sen, sediment PYR40

Figs. 5, $11 \quad$ Lake Long de Liat, sediment PYR55

Figs. 7-9, 13-14 Lake Llebreta, sediment PYR58

Fig. 16 Lake Bachimala Sup., sediment PYR31

Fig. $17 \quad$ Lake Arratille, sediment PYR11

Figs. 6, 18-21 Lake Port Bielh, epilithic EpiPYR28

Fig. 22 Lake Redon, sediment REDOM 

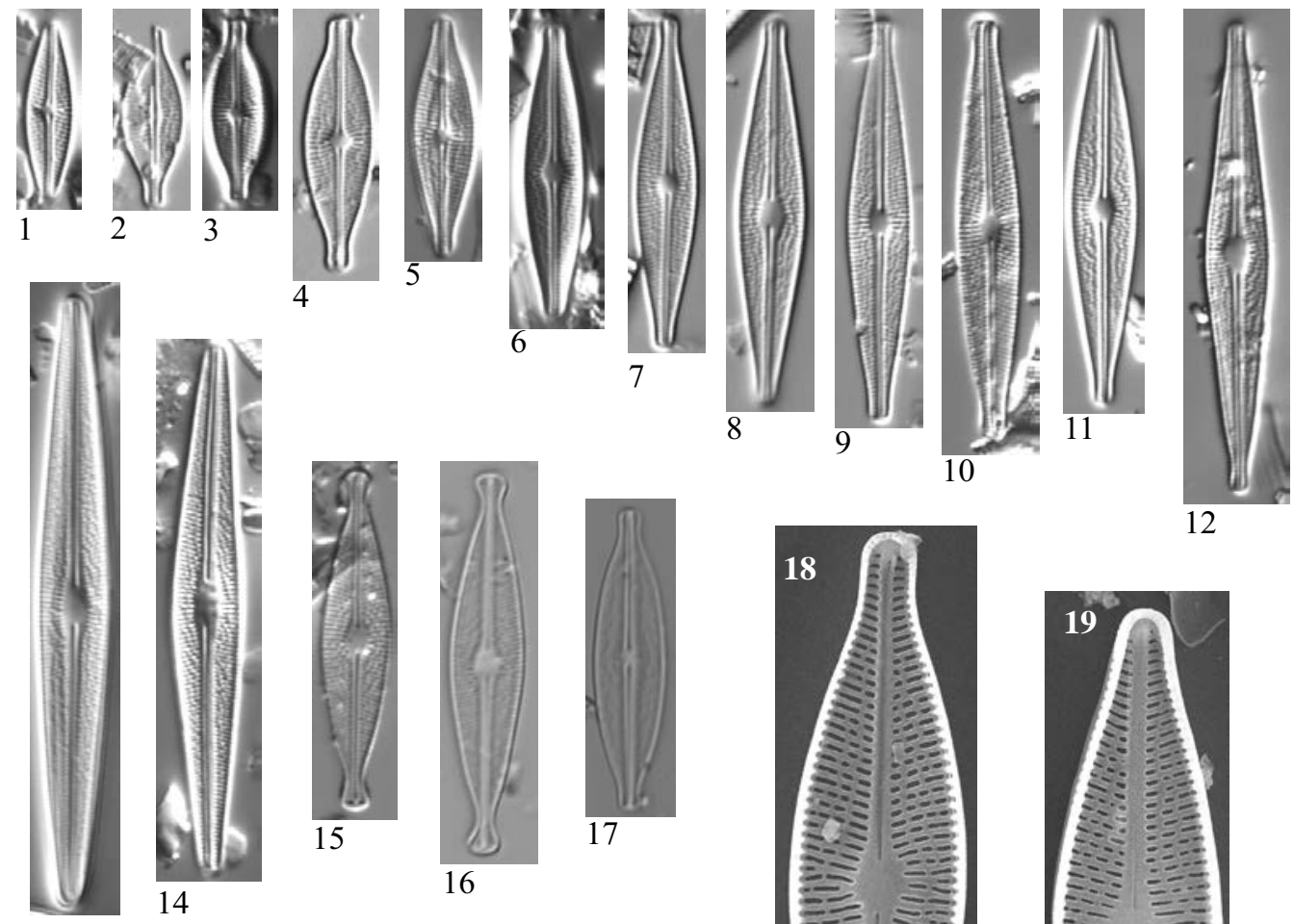

13
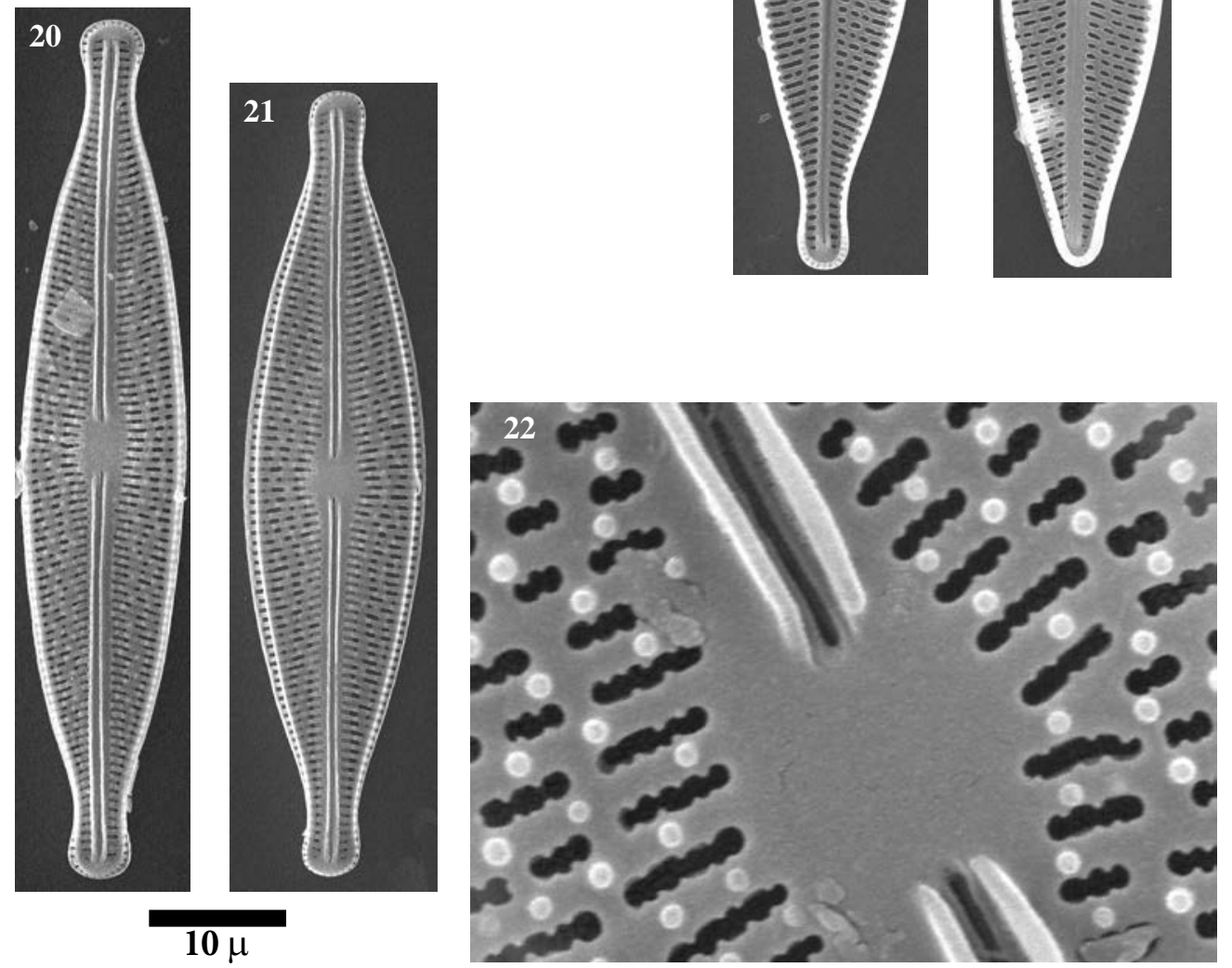

Limnetica, 36 (1): 127-395 (2017) 
Plate $79 \quad$ LM: $x 1500$

SEM: $\mathrm{x} 4000$

Figs. 1-6 Brachysira neoexilis Lange-Bertalot

Fig. 7

Brachysira cf. vitrea (Grunow) Ross

Figs. 1, 2, 5 Lake Redon, sediment REDOM

Fig. 7 Lake Arnales, sediment PYR09

Figs. 3-4, 6 Lake Sen, sediment EpiPYR28 

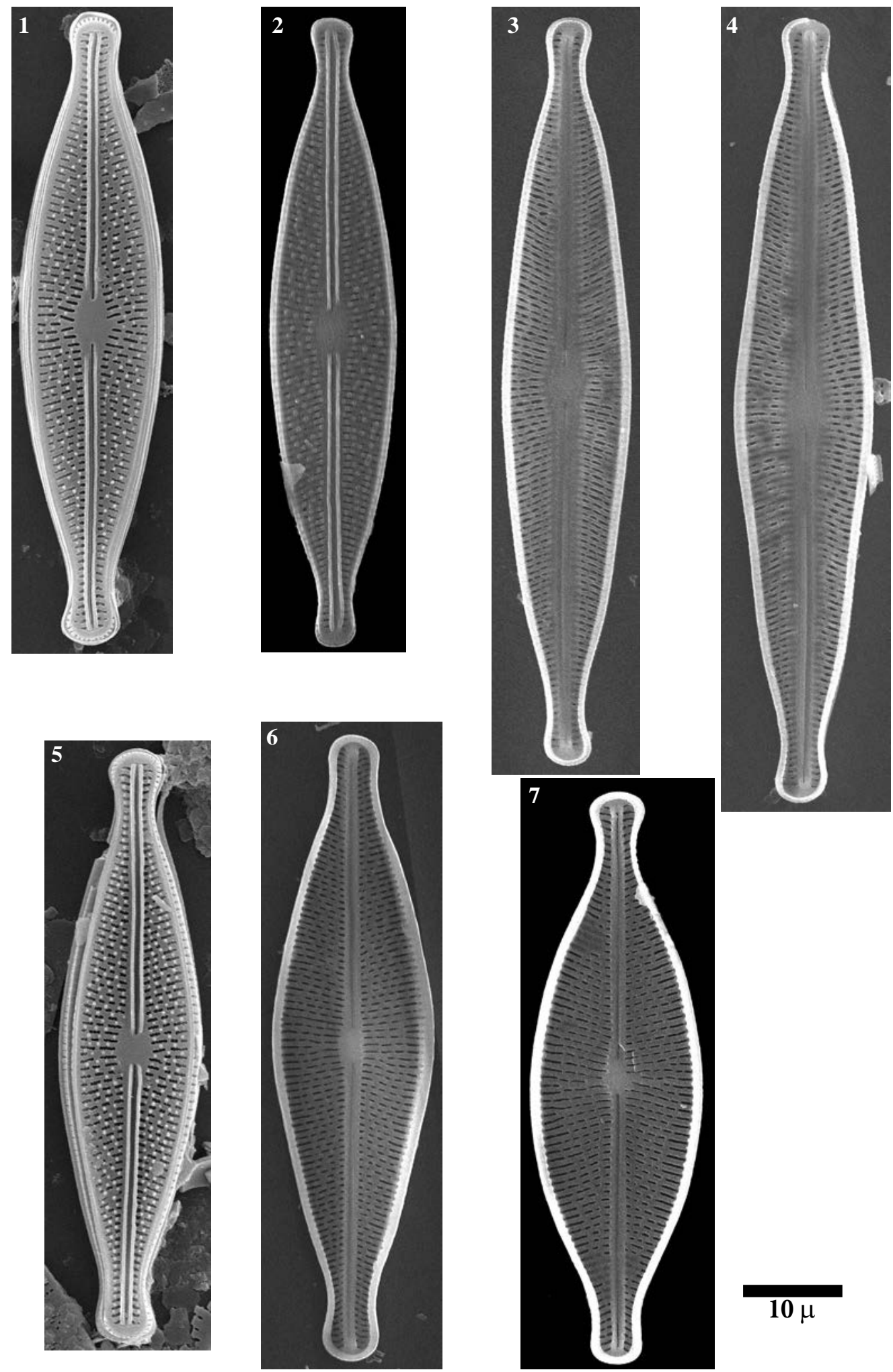

Limnetica, 36 (1): 127-395 (2017) 
Plate $80 \quad$ LM: $x 1500$

SEM: Figs. 3,6 x15000, Fig. 5 x2000

Figs. 1-6 Frustulia crassinervia (Brébisson) Lange-Bertalot et Krammer

Figs. 1, 2 Lake Gelat Bergús, sediment PYR65

Figs. 3, 5-6 Lake Redon, sediment REDOM

Fig. 4 Lake Pica Palomera, sediment PYR52 

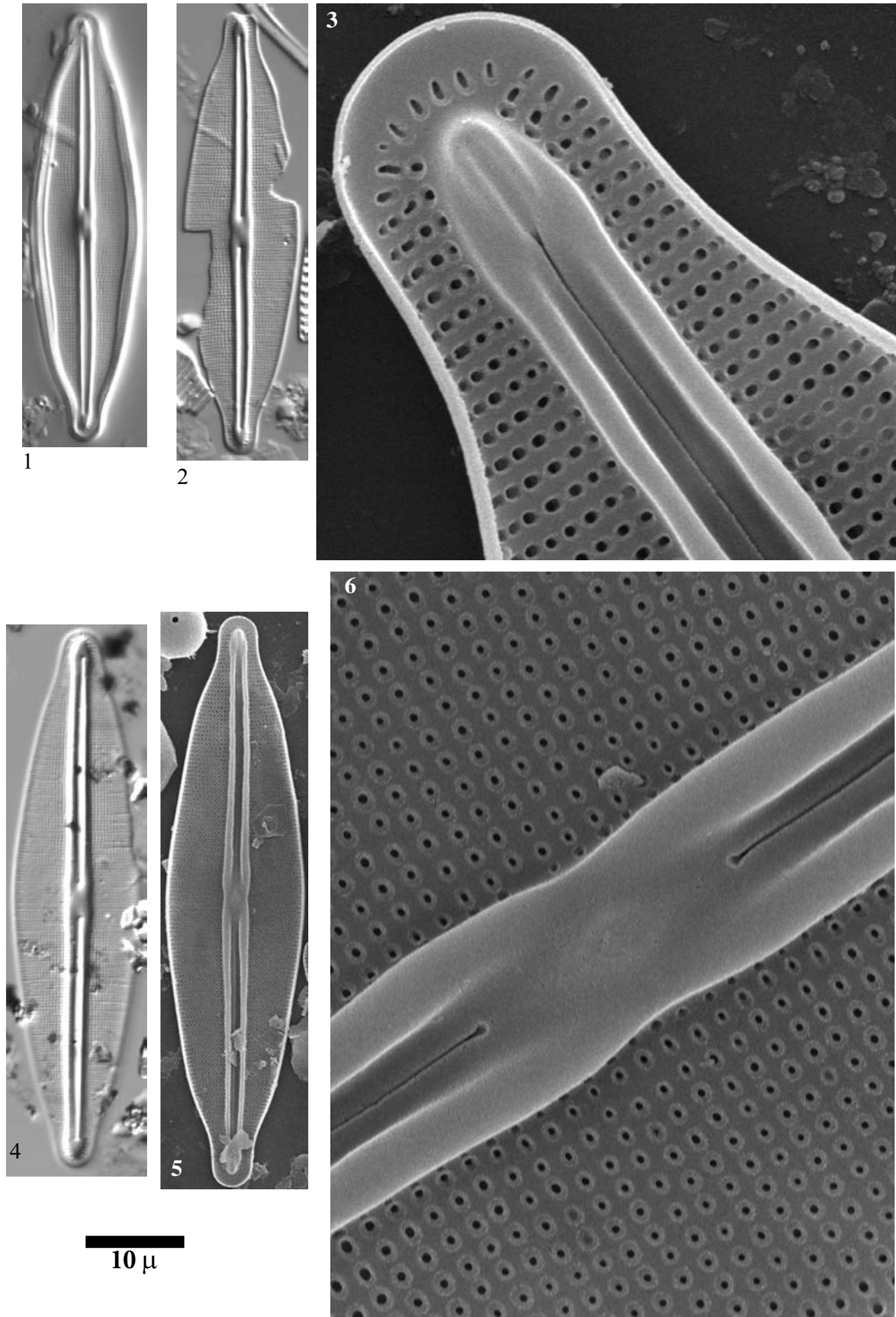
Plate $81 \quad$ LM: $x 1500$

SEM: Fig. 2 x1500, Figs. 3-4 x10000

Figs. 1-4 Frustulia cf. crassinervia (Brébisson) Lange-Bertalot et Krammer

Fig. $1 \quad$ Lake Pica Palomera, sediment PYR52

Figs. 2-4 Lake Mariola, epilithic EpiPYR80 

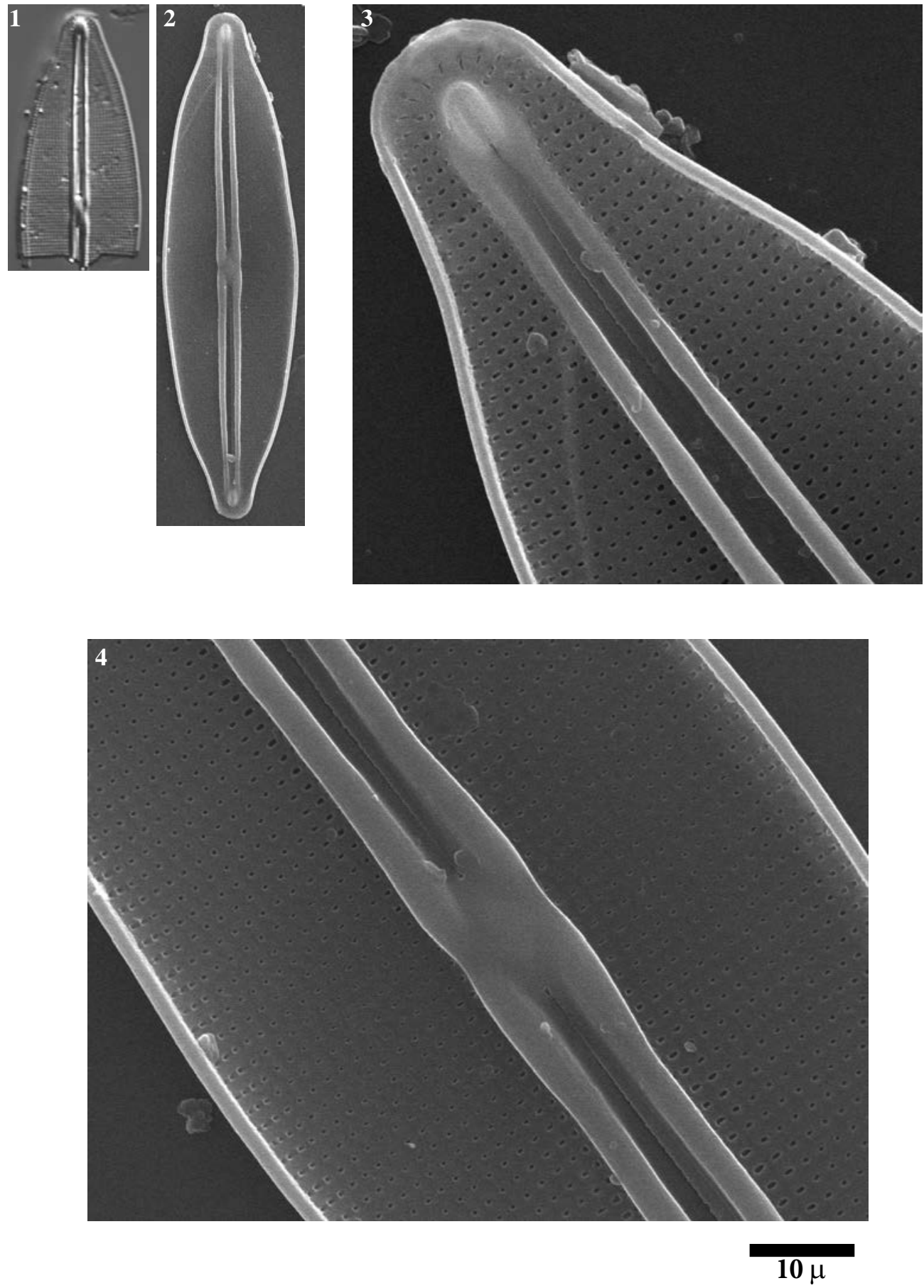
Plate 82 LM: $x 1500$

SEM: Fig. 2 x2000, Figs. 3-4 x5000

Figs. 1-6 Frustulia cf. saxonica Rabenhorst

Figs. 1, 3-5 Lake Senó, epilithic EpiPYR84

Fig. 2 Lake Monges, sediment PYR57

Fig. 6 Lake Bleu de Rabassoles, epilithic EpiPYR112 

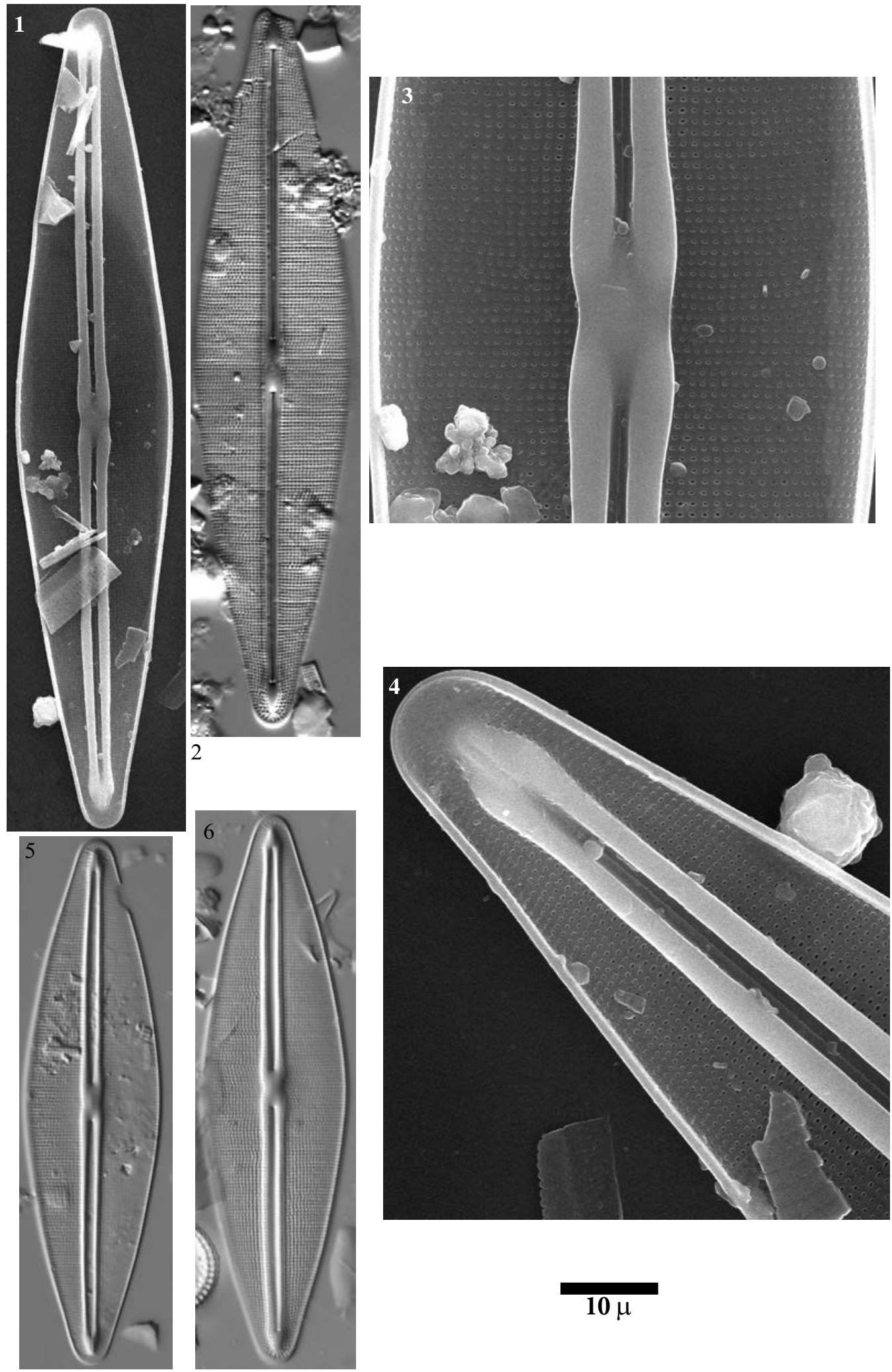

$10 \mu$ 
Plate 83 LM: $x 1500$

SEM: Fig. 2 x1500, Figs. 3-4 x5000

Figs. 1-4 Frustulia erifuga Lange-Bertalot et Krammer

Fig. 5 Amphipleura pellucida (Kützing) Kützing

Fig. 1 Lake Senó, sediment PYR84

Figs. 2-4 Lake Redon, sediment REDOM

Fig. 5 Lake Burg, sediment BURG 1054 

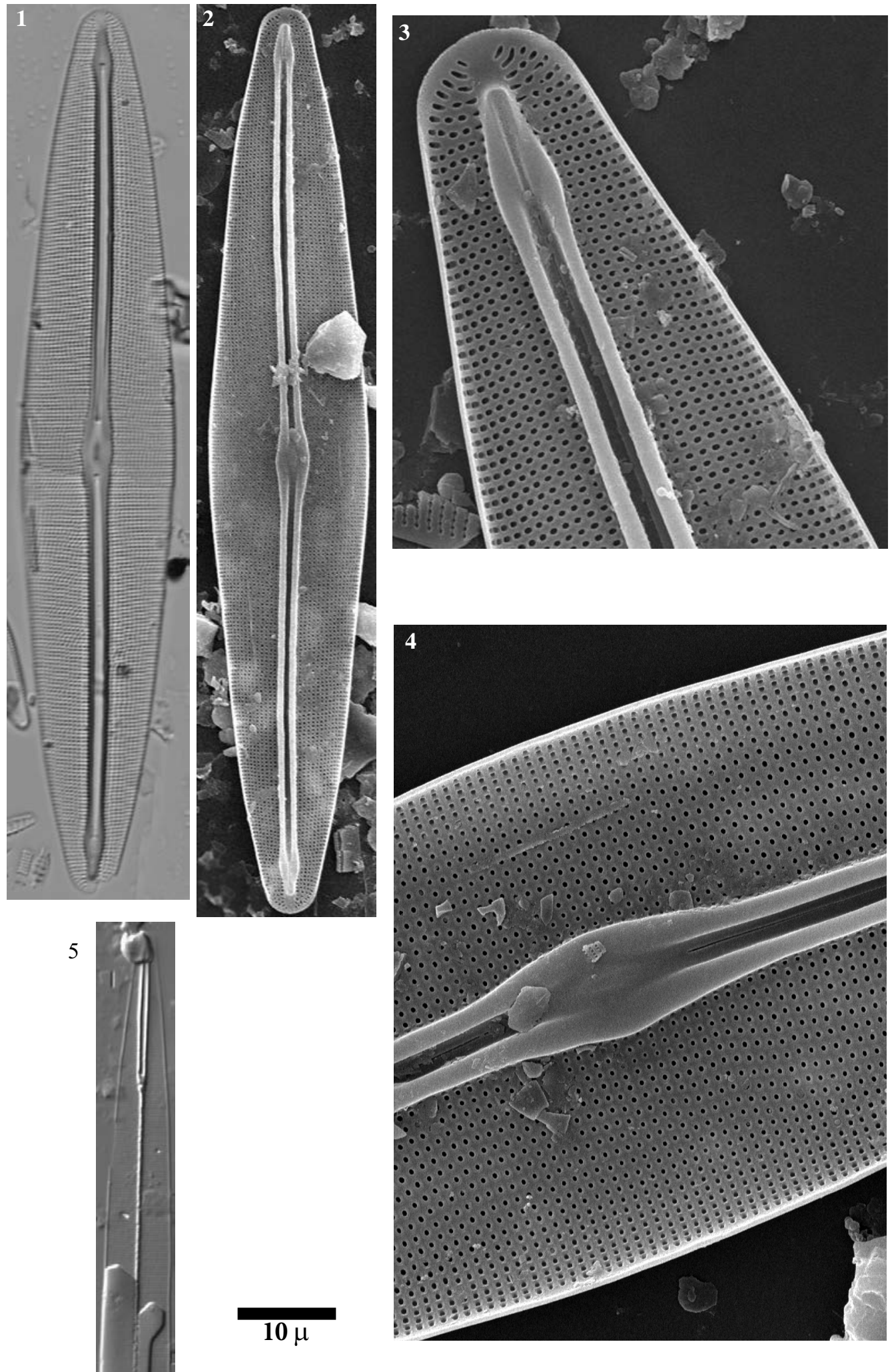

Limnetica, 36 (1): 127-395 (2017) 
Plate 84 LM: $x 1500$

SEM: x3000

Fig. 1

Fig. 2

Figs. 3, 10

Fig. 4

Figs. 5-6

Fig. 7

Figs. 8-9, 11
Diploneis cf. oculata (Brébisson) Cleve

Diploneis cf. peterseni (petersenii) Hustedt

Diploneis cf. modica Hustedt

Diploneis sp. No. 1 Pica Palomera

Diploneis cf. puella (Schumann) Cleve

Diploneis cf. parma Cleve sensu auct nonnull.

Diploneis cf. subovalis Cleve

Fig. 1 Lake Basa de la Mora, sediment PYR32

Figs. 2, 6-7 Lake Sen, sediment PYR40

Fig. 3 Lake Acherito, sediment PYR01

Fig. 4 Lake Pica Palomera, sediment PYR52

Fig. 5 Lake Arnales, sediment PYR09

Fig. 8 Lake Eriste, sediment PYR43

Fig. 9 Lake Arratille, sediment PYR11

Figs. 10-11 Lake Laurenti, sediment PYR111 


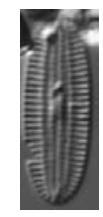

1
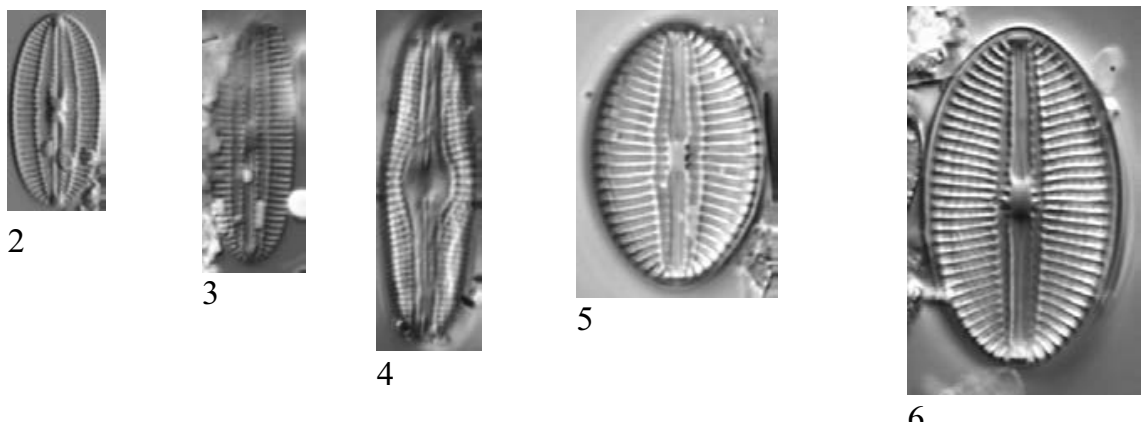

6
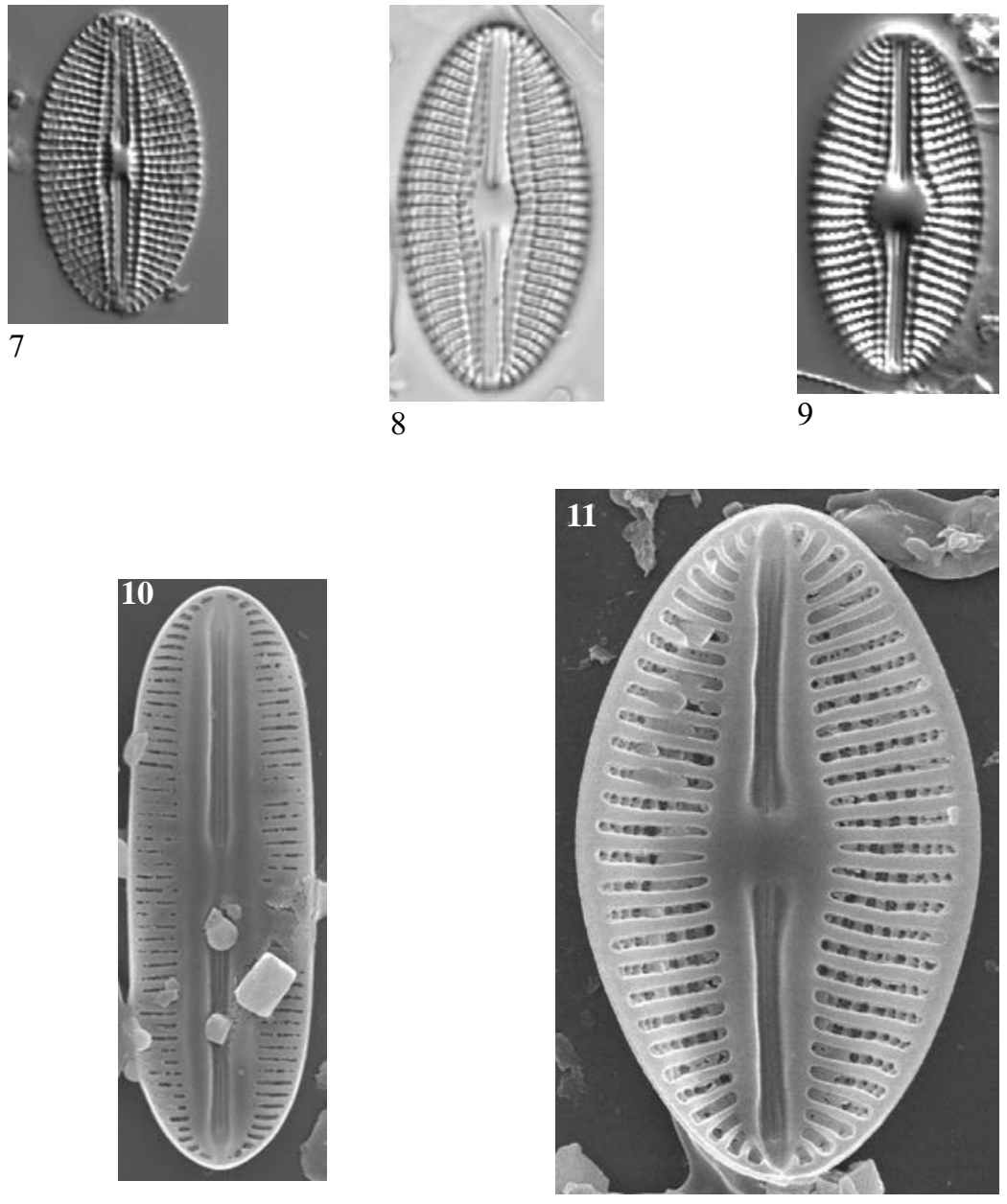

$10 \mu$ 
LM: x1500

Figs. 1-2

Figs. 3-4

Fig. 5

Figs. 6-11, 17,18

Figs. 12-15

Fig. 16

Fig. 19

Fig. 20

Figs. 21-23

Fig. 24

Figs. 25-27

Fig. 28

Figs. 1, 2

Fig. 3

Figs. 4, 21-23

Fig. 5

Figs. 6-11, 17-18, 28

Fig. 12

Fig. 13

Fig. 14

Fig. 15

Fig. 16

Fig. 19

Fig. 20

Figs. 24-25

Figs. 26-27
Caloneis sp. No. 1 Munia

Caloneis cf. lancettula (Schulz) Lange-Bertalot \& Witkowski

Caloneis cf. vasilyevae Lange-Bertalot, Genkal \& Vechov

Caloneis sp. No. 2 Posets

Caloneis silicula (Ehrenberg) Cleve sensu lato

Caloneis sp. No. 3 Posets

Caloneis alpestris (Grunow) Cleve

Caloneis sp. No. 4 Burg

Caloneis sp. No. 5 Acherito

Caloneis cf. tenuis (Gregory) Krammer

Caloneis cf. undulata (Gregory) Krammer

Caloneis cf. lauta Carter

Lake La Munia Sup., sediment PYR20

Lake Burg, sediment BURG 616

Lake Acherito, sediment PYR01

Lake Pica Palomera, sediment PYR52

Lake Posets, sediment PYR42

Lake Burg, sediment BURG 1216

Lake Estom, sediment PYR15

Lake Col d Arratille, sediment PYR12

Lake Burg, sediment BURG 703

Lake Pixón, sediment PYR44

Lake Arratille, sediment PYR11

Lake Burg

Lake Montoliu, epilithic EpiPYR54

Lake Long de Liat, sediment PYR55 

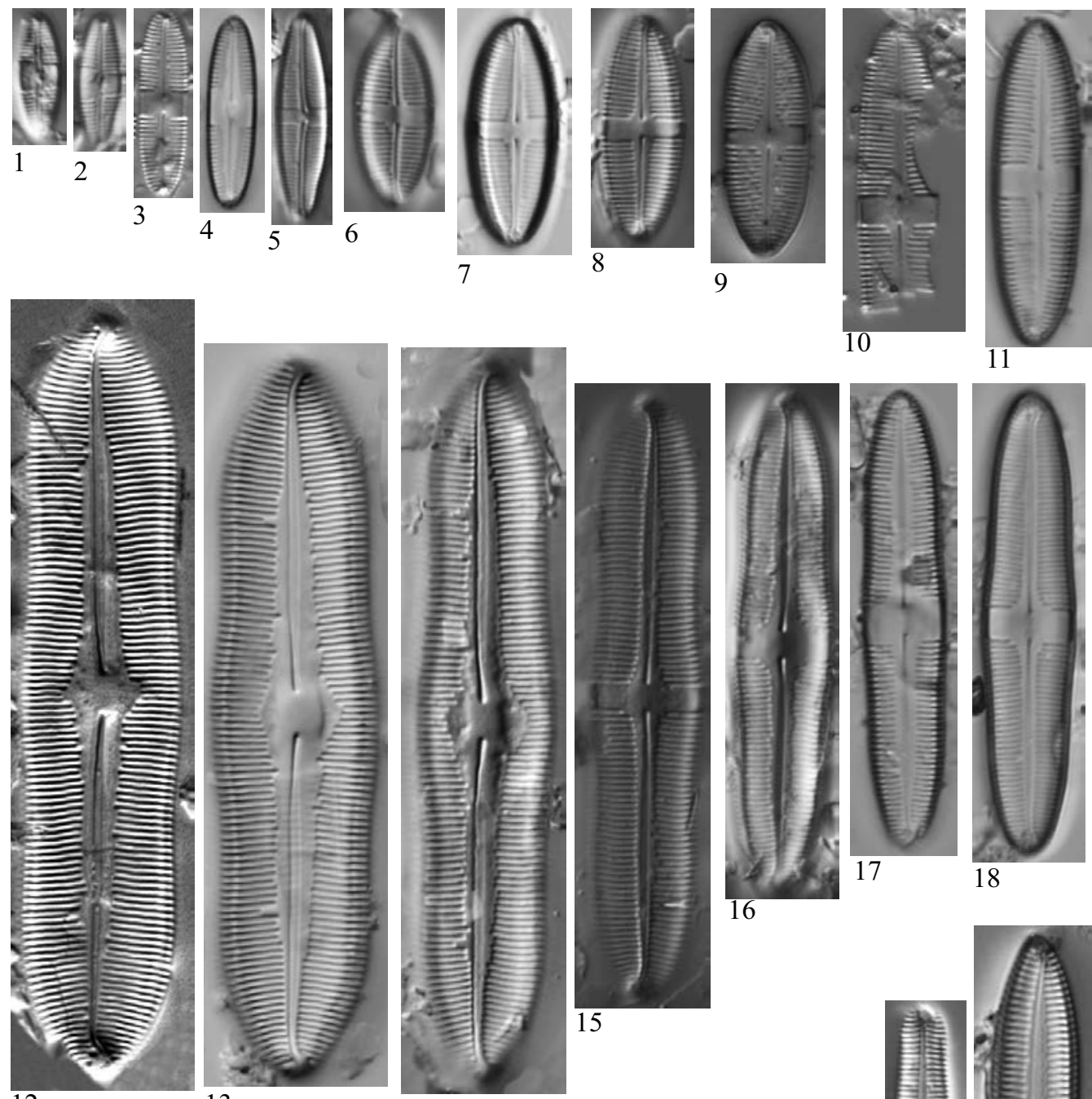

12

13

14
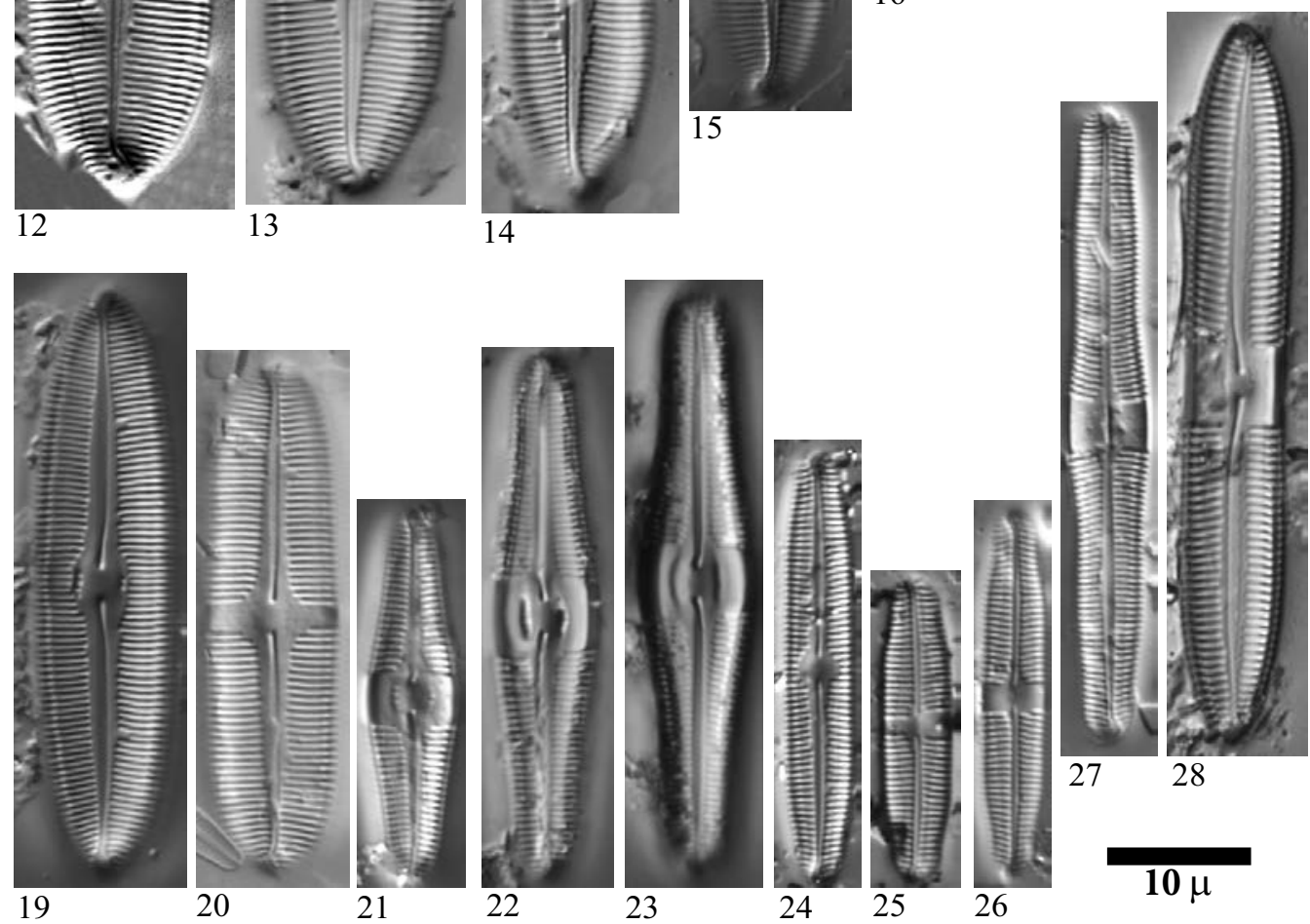

Limnetica, 36 (1): 127-395 (2017) 
SEM: Figs. 14,16 x3000, Fig. 18 x10000, Fig. 19 x15000

Fig. 1

Pinnularia sinistra Krammer

Figs. 2-10, 15 Pinnularia subcapitata Gregory 18-19

Fig. 11

Pinnularia sp. No. 1 Posets

Figs. 12-14 Pinnularia cf. subanglica Krammer

Figs. 16-17 Pinnularia cf. rumrichae Krammer

Fig. 1 Lake Llosás, sediment PYR46

Fig. $2 \quad$ Lake Burg

Figs. 3, 11-12, Lake Posets, sediment PYR42

17

Fig. $4 \quad$ Lake Senó, epilithic EpiPYR84

Figs. 5, $10 \quad$ Lake Senó, sediment PYR84

Fig. $6 \quad$ Lake Burg, sediment BURG 958

Fig. 7 Lake Burg, sediment BURG 968

Fig. 9 Lake Redon, sediment REDOM

Fig. 13 Lake Arnales, sediment PYR09

Fig. 14 Lake Garbet, sediment PYR81

Figs. 16, 19 Lake Mariola, epilithic EpiPYR80 


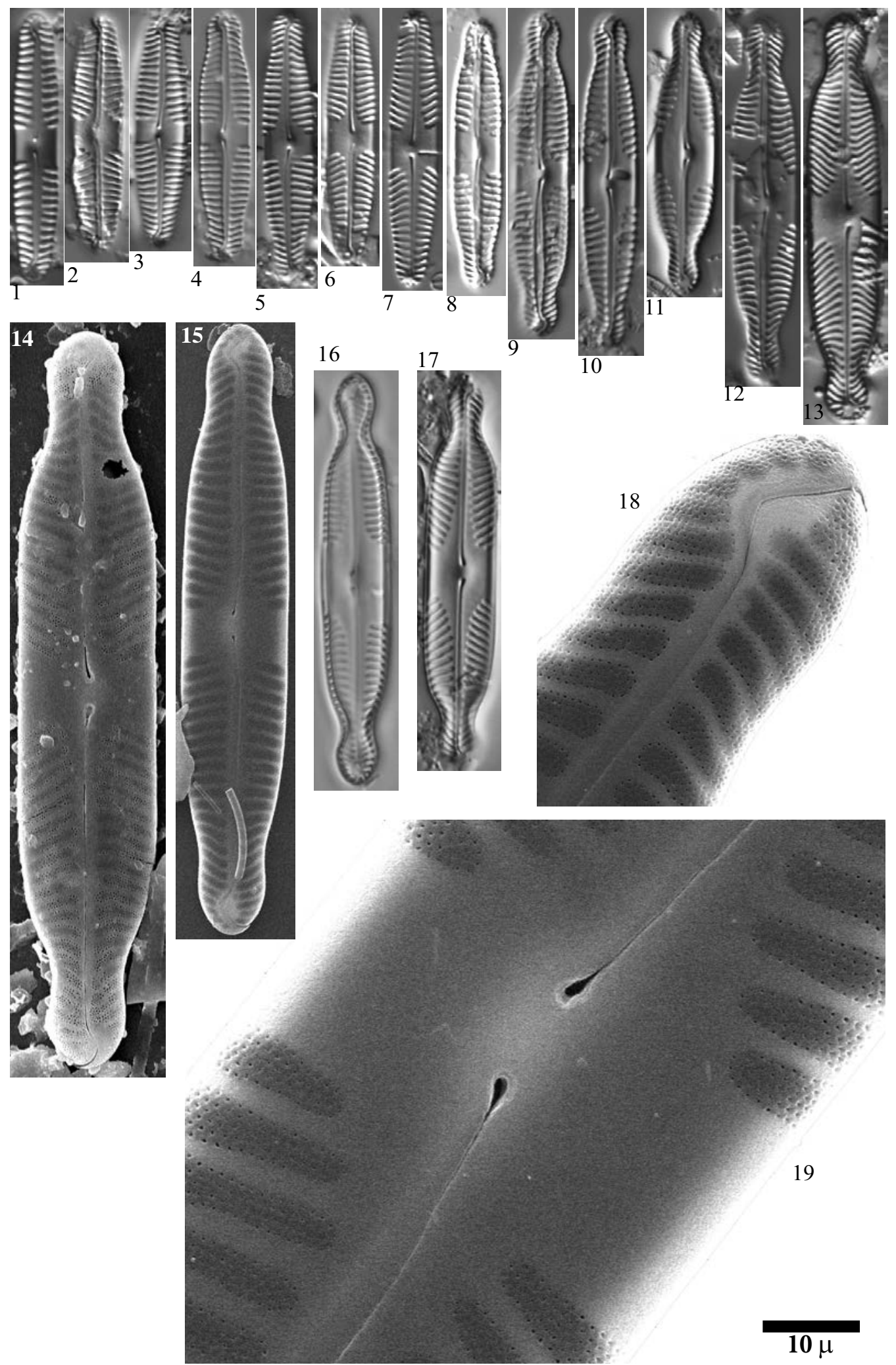


SEM: Fig. 13 x10000, Fig. 14 x300, Figs. 16-18 x4500

Figs. 1-2

Fig. 3

Figs. 4-10 17-18

Fig. 11

Figs. 12-14

Figs. 15-16

Figs. 1-3, 5

Fig. 4

Fig. 6

Fig. 7

Fig. 8

Fig. 9

Fig. 10

Fig. 11

Fig. 12-14, 16

Fig. 15

Figs. 17-18
Pinnularia sp.

Pinnularia sp.

Pinnularia cf. brebissonii var. minuta Krammer

Pinnularia sp. No. 3 Plan

Pinnularia sp. No. 4 Mariola

Pinnularia sp. No. 6 Estelat

Lake Posets, sediment PYR42

Lake Baiau Superior, sediment PYR77

Lake Arratille, sediment PYR11

Lake Illa, sediment PYR66

Lake Ensangents Sup., sediment PYR106

Lake Burg

Lake Burg, sediment BURG 1187

Lake Plan, sediment PYR69

Lake Mariola, sediment PYR80

Lake Estelat, sediment PYR120

Lake Redon, sediment REDOM 


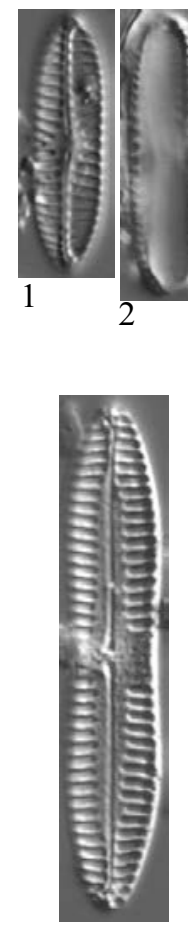

12
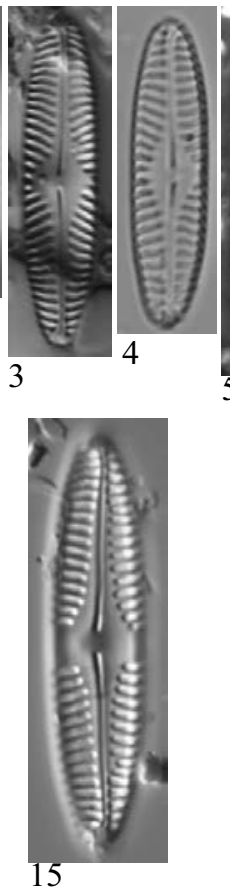

14
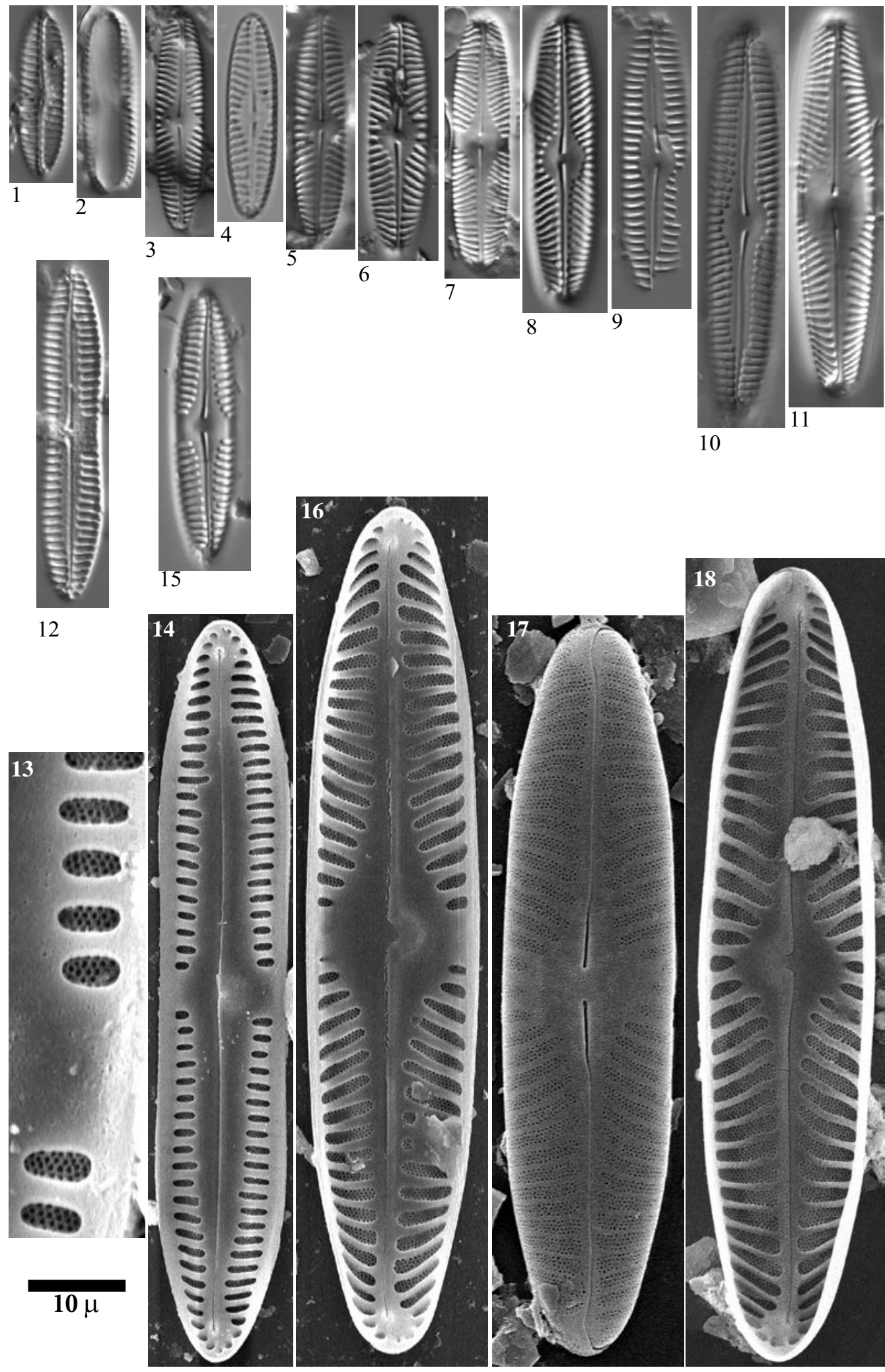

18
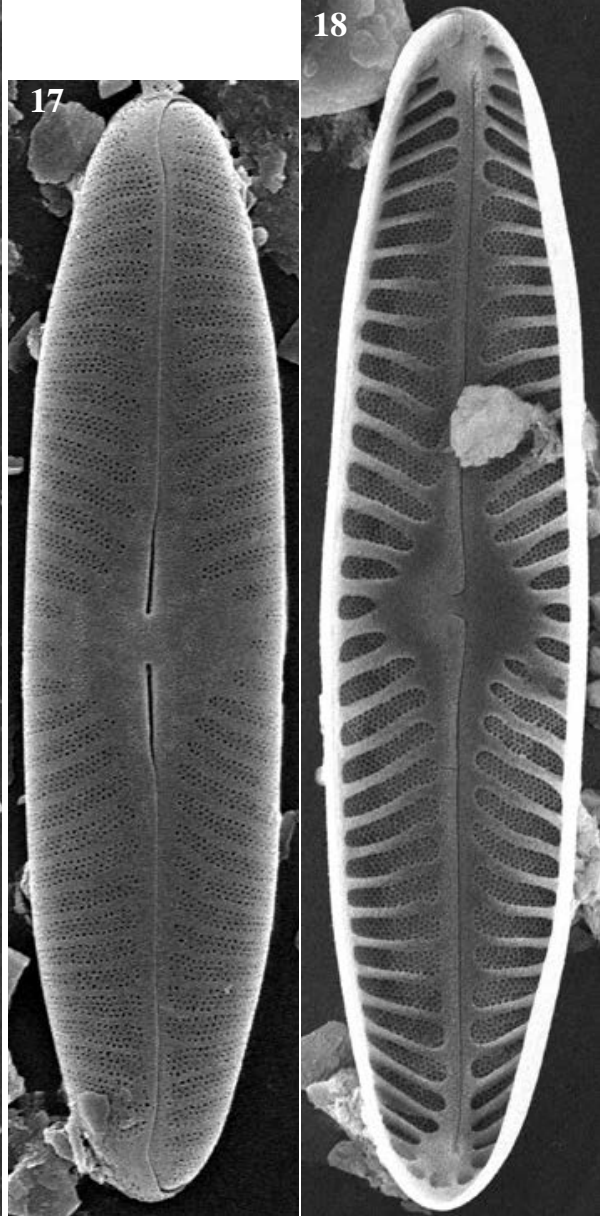
SEM: Fig. 15 x3000, Fig. 23 x7500, Fig. 24 x10000

Fig. 1

Figs. 2-3

Figs. 4-6

Fig. 7

Fig. 8

Fig. 9

Fig. 10

Figs. 11-12

Fig. 13

Fig. 14

Figs. 15-20 $23-24$

Fig. 21

Fig. 22
Hygropetra balfouriana (Grunow ex Cleve) Krammer \& Lange Bertalot

Pinnularia cf. laucensis Lange-Bertalot, Rumrich \& Krammer

Pinnularia sp. No. 12 Estelat, aff. perirrorata

Pinnularia cf. kuetzingii Krammer

Pinnularia sp. No. 7 Romedo

Pinnularia sp. No. 8 Burg

Pinnularia subinterrupta Krammer \& Schroeter

Pinnularia sp. No. 2 Illa

Pinnularia sp

Pinnularia sp. 15 Burg

Pinnularia microstauron var. nonfasciata Krammer

Pinnularia sp. No. 5 Mora

Pinnularia sp. No. 14 Burg, aff. Pinnularia divergens Smith

Fig. 1 Lake Eriste, sediment PYR43

Figs. 2-3, $20 \quad$ Lake Negre, sediment PYR79

Fig. $4 \quad$ Lake Inf. de la Gallina, sediment PYR87

Figs. 5, 18, Lake Mariola, epilithic EpiPYR80

23-24

Fig. 6 Lake Estelat, sediment PYR120

Fig. 7 Lake Inf. de la Gallina, epilithic EpiPYR87

Fig. 8 Lake Romedo de Dalt, epilithic EpiPYR85

Fig. 9 Lake Burg, sediment BURG 985

Fig. $10 \quad$ Lake Illa, epilithic EpiPYR66

Figs. 11-12, Lake Illa, sediment PYR66

$16-18$

Figs. 13-14 Lake Burg, sediment BURG 918

Fig. 19 Lake Posets, sediment PYR42

Fig. 21 Lake Basa de la Mora, sediment PYR32

Fig. 22 Lake Burg, sediment BURG 960 

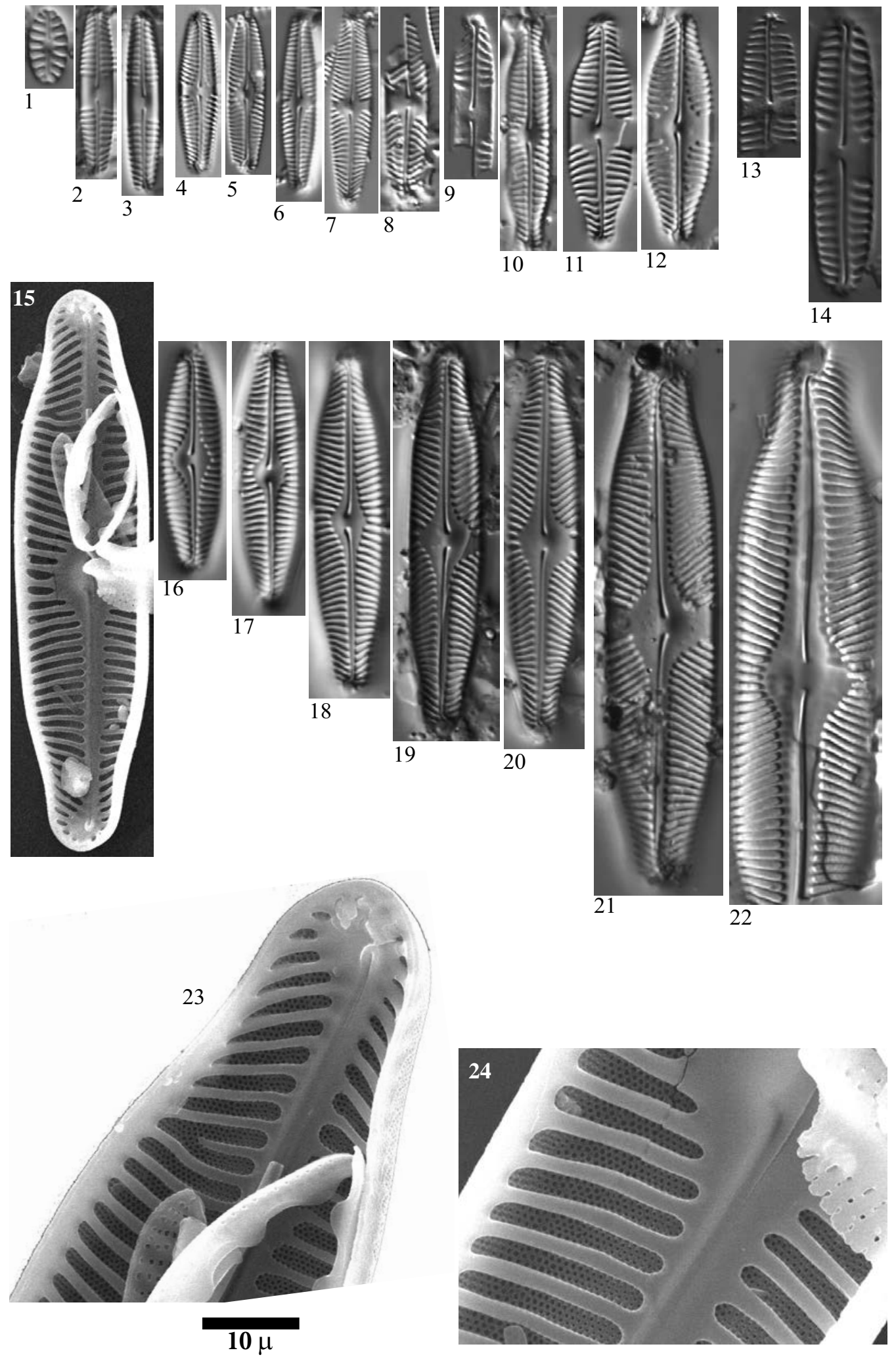

Limnetica, 36 (1): 127-395 (2017) 
SEM: Fig. 7 x8000, Fig. 8 x2000

Figs. 1-3

Fig. 4

Figs. 5-8

Fig. 1

Fig. 2

Fig. 3

Fig. 4

Fig. 5

Fig. 6

Figs. 7-8
Pinnularia grunowii Krammer

Pinnularia sp. No. 13 Albe

Pinnularia septentrionalis Krammer

Lake Burg

Lake Burg, sediment BURG 917

Lake Burg, sediment BURG 796

Lake Albe, sediment PYR96

Lake Sen, sediment PYR40

Lake Posets, sediment PYR42

Lake Laurenti, sediment PYR111 

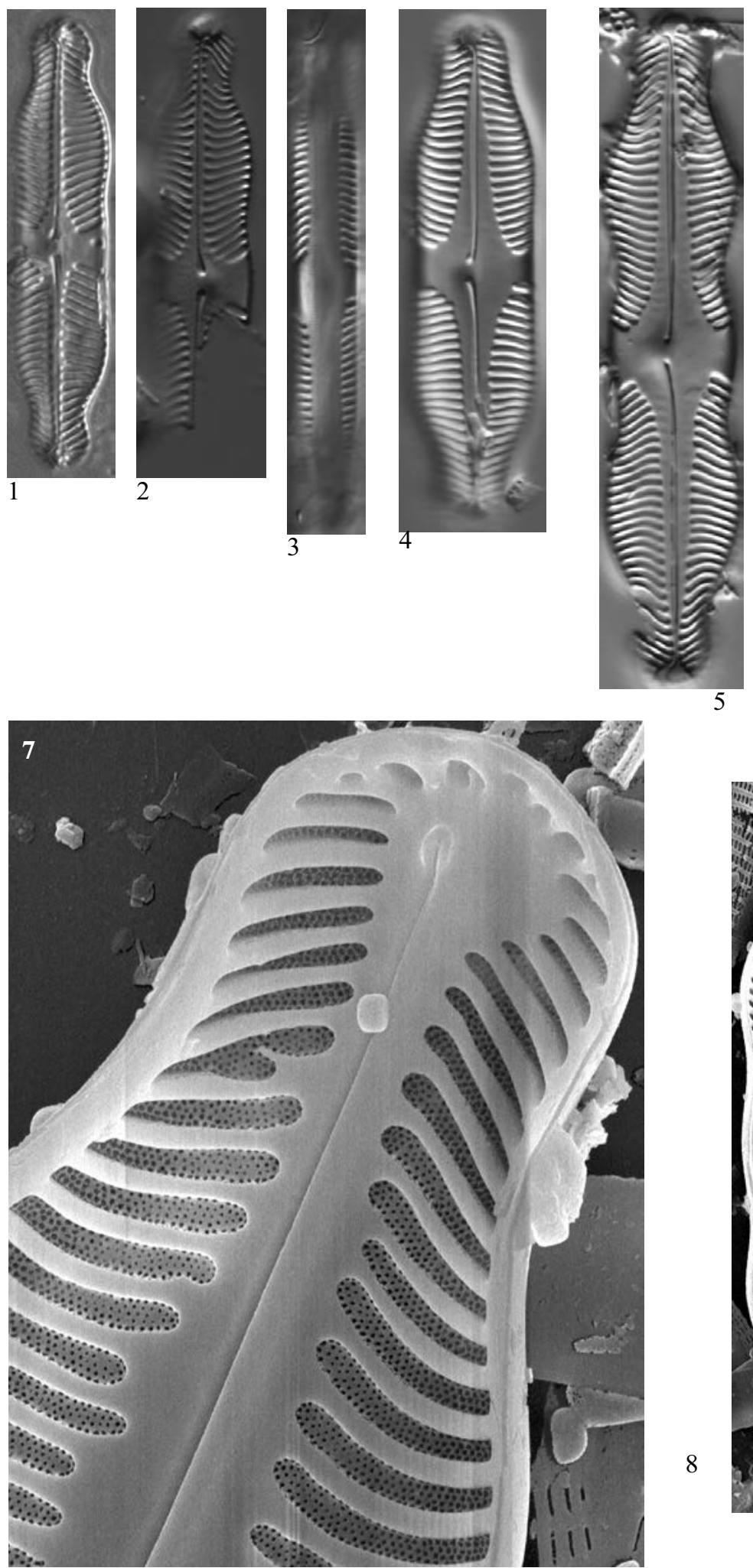

6
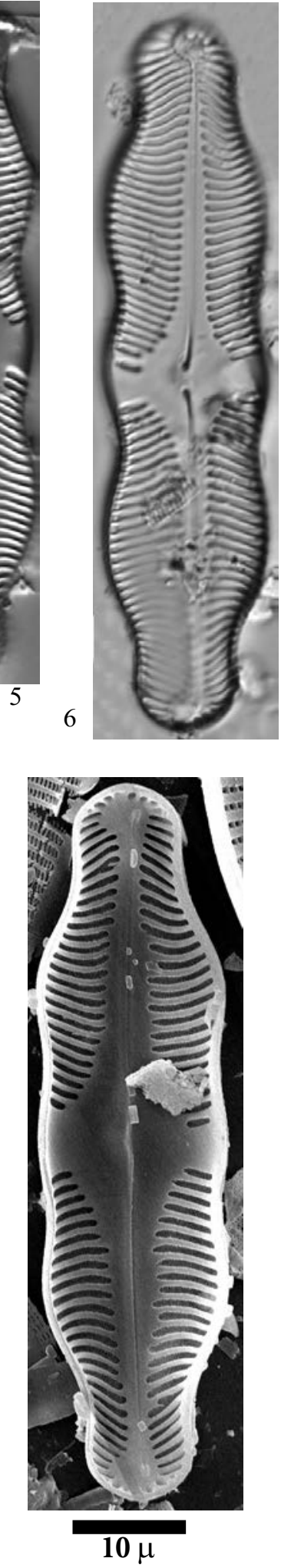

Limnetica, 36 (1): 127-395 (2017) 
Plate $90 \quad$ LM: $\mathrm{x} 1500$

Figs. 1-4 Pinnularia borealis Ehrenberg

Fig. 5 Pinnularia cf. lata (Brébisson) Smith

Fig. 6 Pinnularia sp. No. 9 Laquettes, aff. subgibba Krammer

Fig. $7 \quad$ Pinnularia cf. subgibba Krammer

Figs. 8-9 Pinnularia sp. No. 10 Pica Palomera, aff. pseudogibba Krammer

Fig. $10 \quad$ Pinnularia sp. No. 11 Trebens, aff. tirolensis (Metzeltin \& Krammer) Krammer

Fig. 1 Lake Burg

Fig. 2 Lake Negre, sediment PYR79

Fig. 3 Lake Sotllo, epilithic EpiPYR89

Fig. 4 Lake Burg, sediment BURG 1057

Fig. 5 Lake Burg, sediment BURG 1195

Fig. 6 Lake Cap Long, sediment PYR27

Fig. 7 Lake Burg, sediment BURG 807

Fig. 8 Lake Pica Palomera, sediment PYR52

Fig. 9 Lake Senó, sediment PYR84

Fig. 10 Lake Trebens, sediment PYR114 


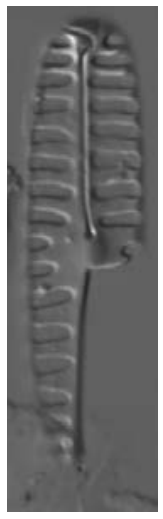

1

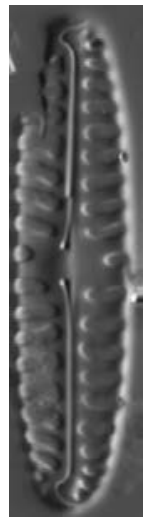

2

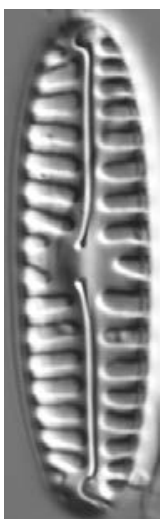

3
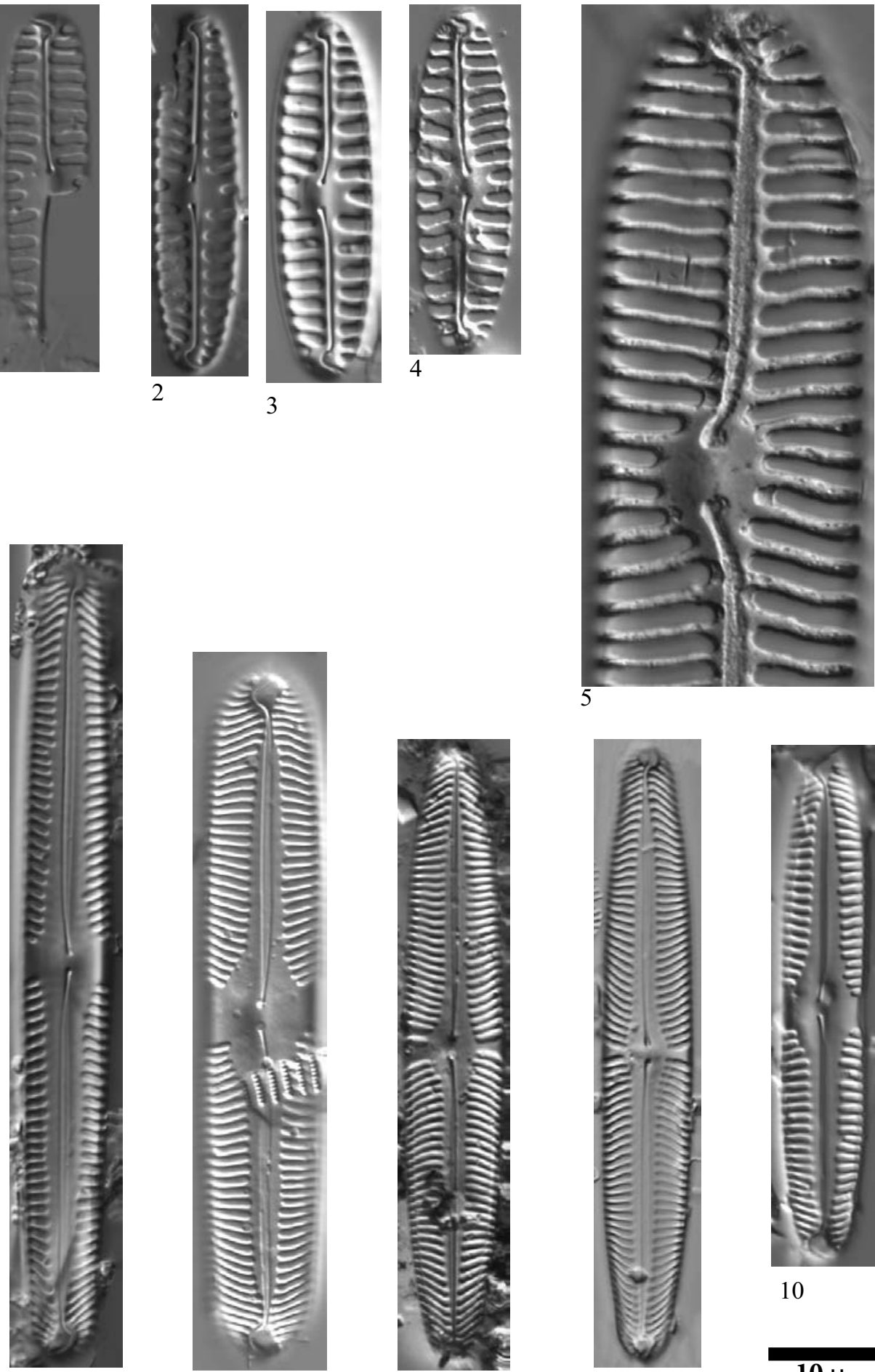

6

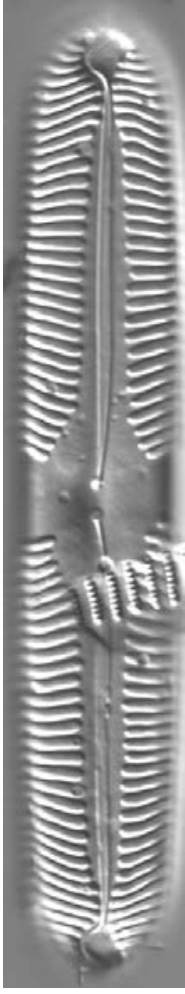

7

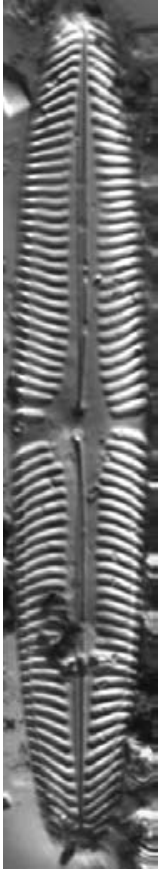

8

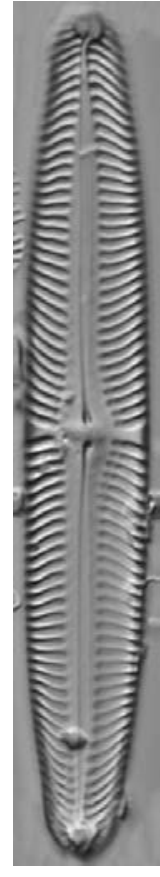

9

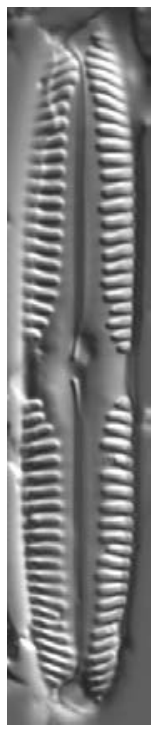

10

$10 \mu$ 
Plate 91 LM: Figs. 1-3 x1500, Fig. 4 x800

Fig. $1 \quad$ Pinnularia sp.

Figs. 2-3 Pinnularia cf. viridis (Nitzsch) Ehrenberg

Fig. $4 \quad$ Pinnularia cf. latevittata Cleve

Fig. 1 Lake Gelat Bergús, sediment PYR65

Fig. 2 Lake Senó, sediment PYR84

Fig. 3 Lake Burg

Fig. 4 Lake Bersau, sediment PYR03 


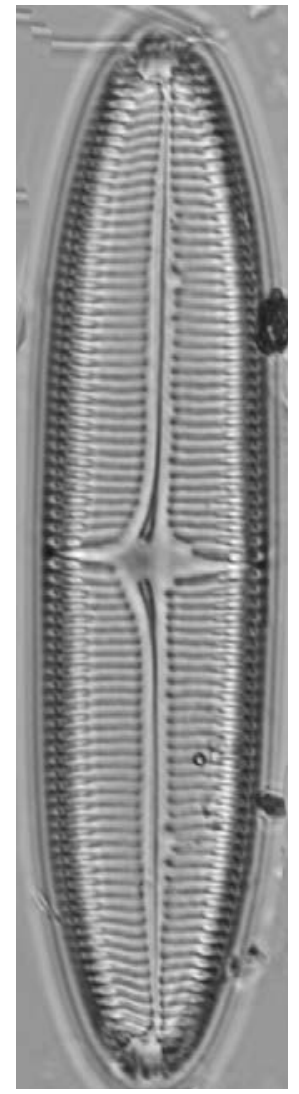

1
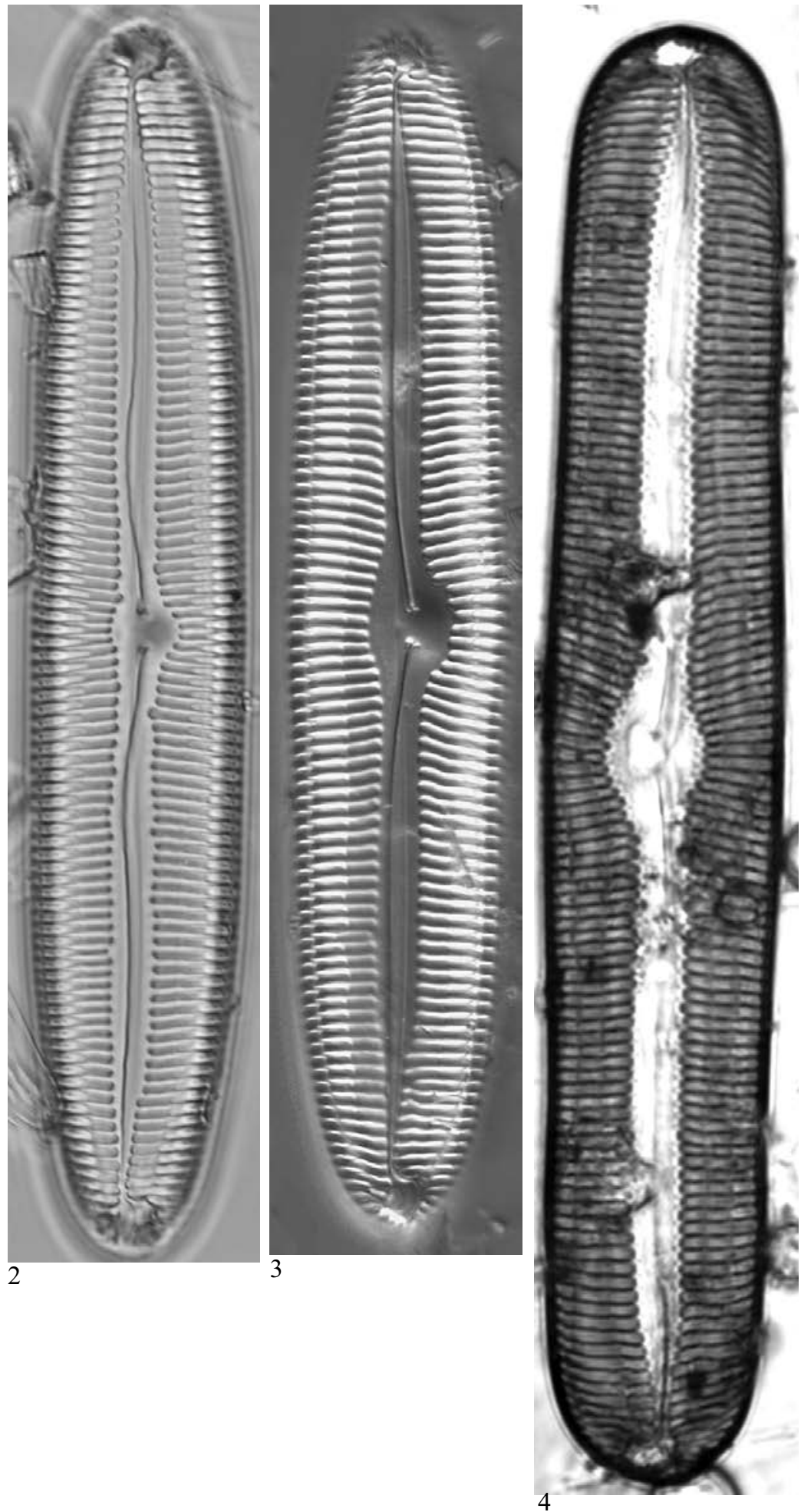
LM: x1500

Fig. 1

Fig. 2

Fig. 3

Fig. 4

Figs. 5-6

Figs. 7-9

Fig. 1

Fig. 2

Fig. 3

Fig. 4

Figs. 5-6

Fig. 7

Figs. 8-9
Pinnularia cf. complexa Krammer

Pinnularia cf. brebissonii var. acuta Cleve-Euler

Pinnularia cf. divergens var. sublinearis Cleve

Pinnularia platycephala (Ehrenberg) Cleve

Pinnularia acuminata Smith

Pinnula sp. 16 Burg, aff. P. divergens

Lake Bersau, epilithic EpiPYR03

Lake Pondiellos Sup., sediment PYR08

Lake Plan, sediment PYR69

Lake PYR128

Lake Illa, sediment PYR66

Lake Burg, sediment BURG 838

Lake Burg, sediment BURG 869 

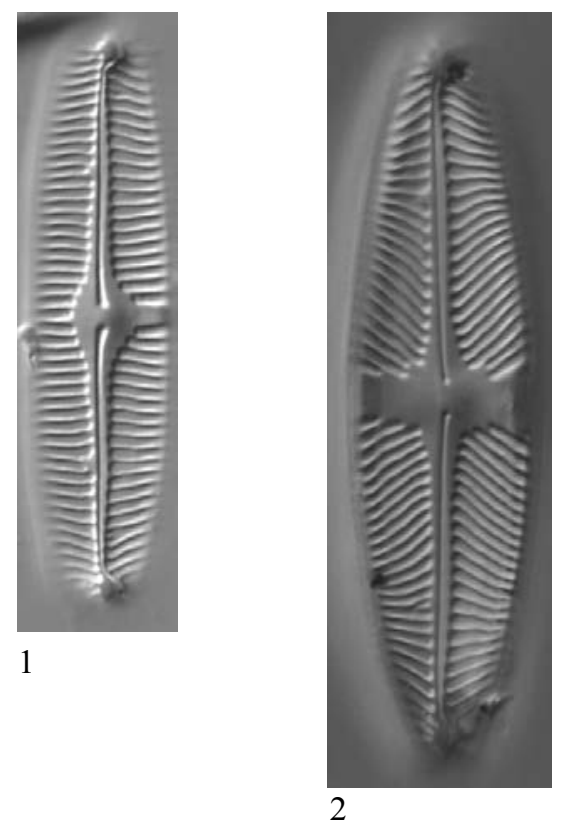

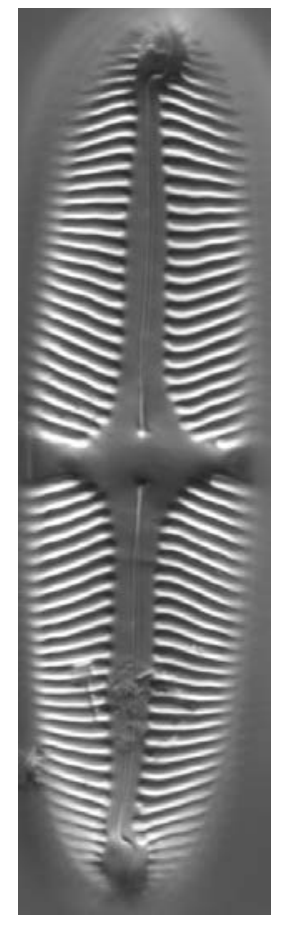

3

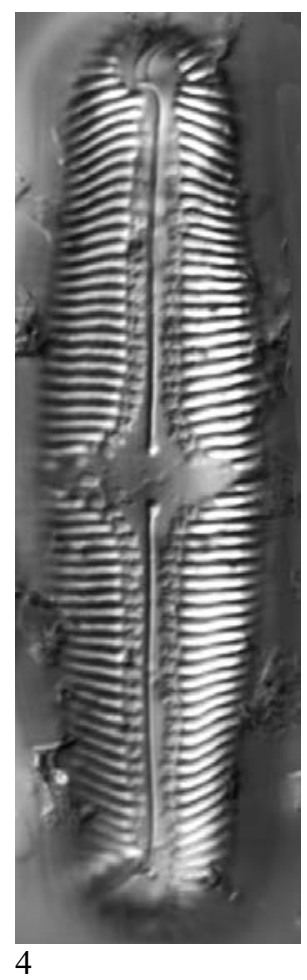

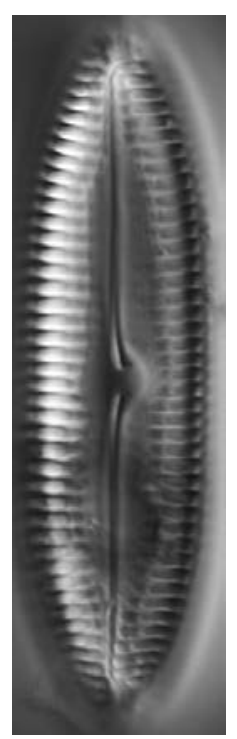

5

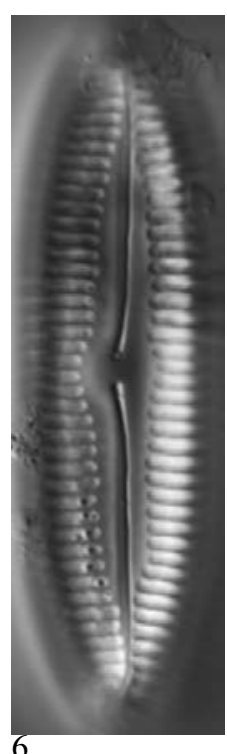

6

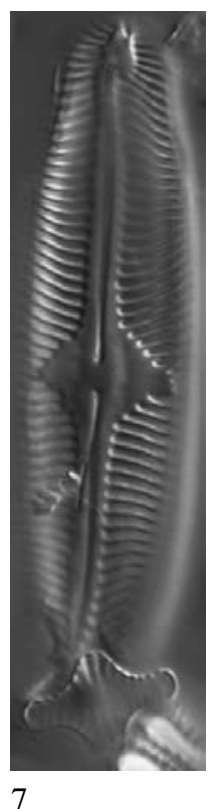

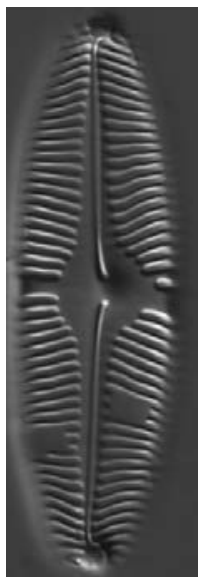

8

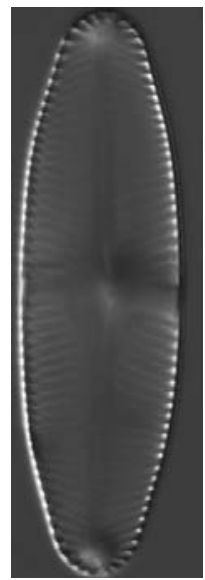

9

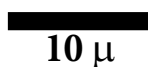


Plate $93 \quad$ LM: $\times 1500$

Fig. 9 x5000, Fig. 10 x4000, Figs. 11,13 x10000, Fig. 12 x6000

Fig. $1 \quad$ Cymbella cf. parva (Smith) Kirchner

Figs. 2-13 Cymbella parva (Smith) Kirchner

Figs. 1, 5, 7 Lake Sen, sediment PYR40

Fig. 2 Lake Acherito, sediment PYR01

Figs. 3, 6, 8 Lake Arratille, sediment PYR11

Figs. 4, 9-13 Lake Roumassot, epilithic EpiPYR04 

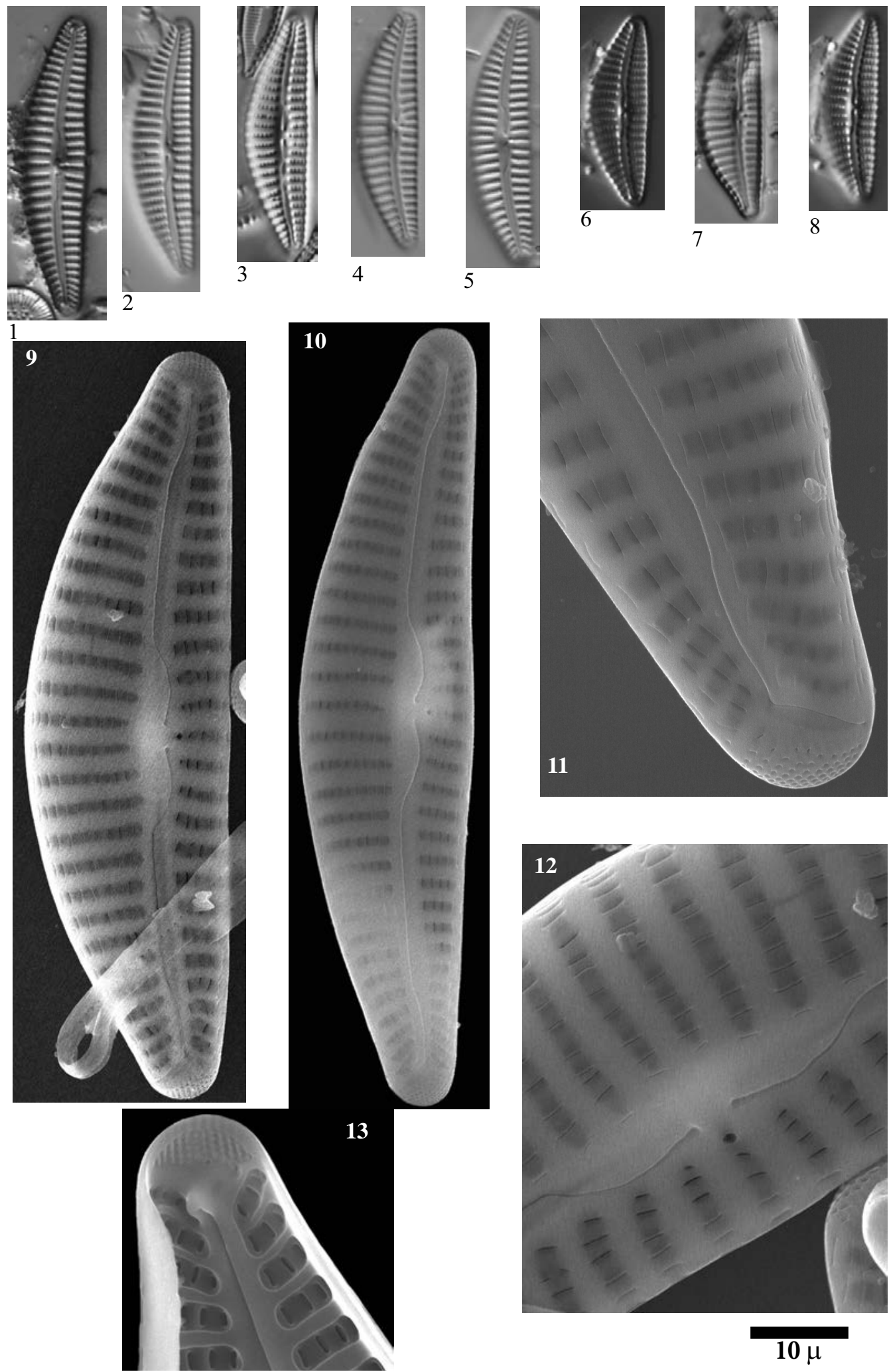

13

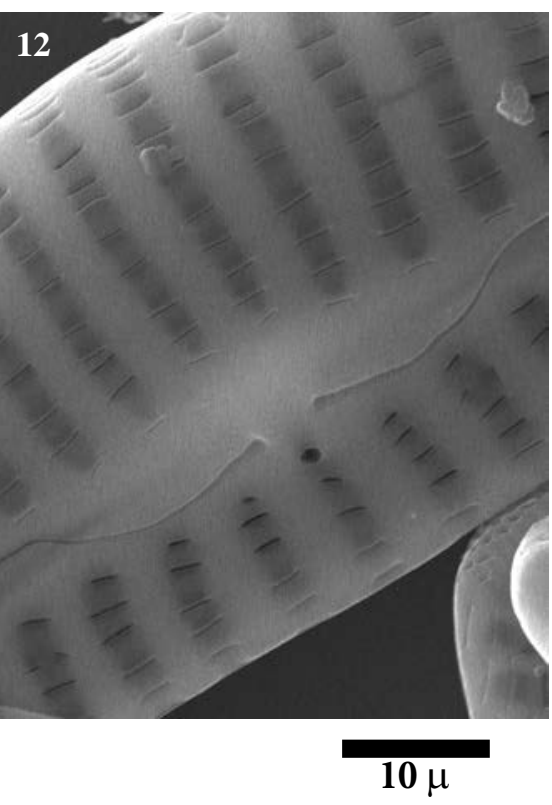

Limnetica, 36 (1): 127-395 (2017) 
Plate $94 \quad$ LM: $x 1500$

SEM: Figs. 1-2,13 x3000, Fig. 3 x10000, Figs. $4-5$ x6000

Cymbella parva (Smith) Kirchner

Figs. 1, 3, $5 \quad$ Lake Gran de Mainera, epilithic EpiPYR70

Figs. 2, $4 \quad$ Lake Roumassot, sediment PYR04 

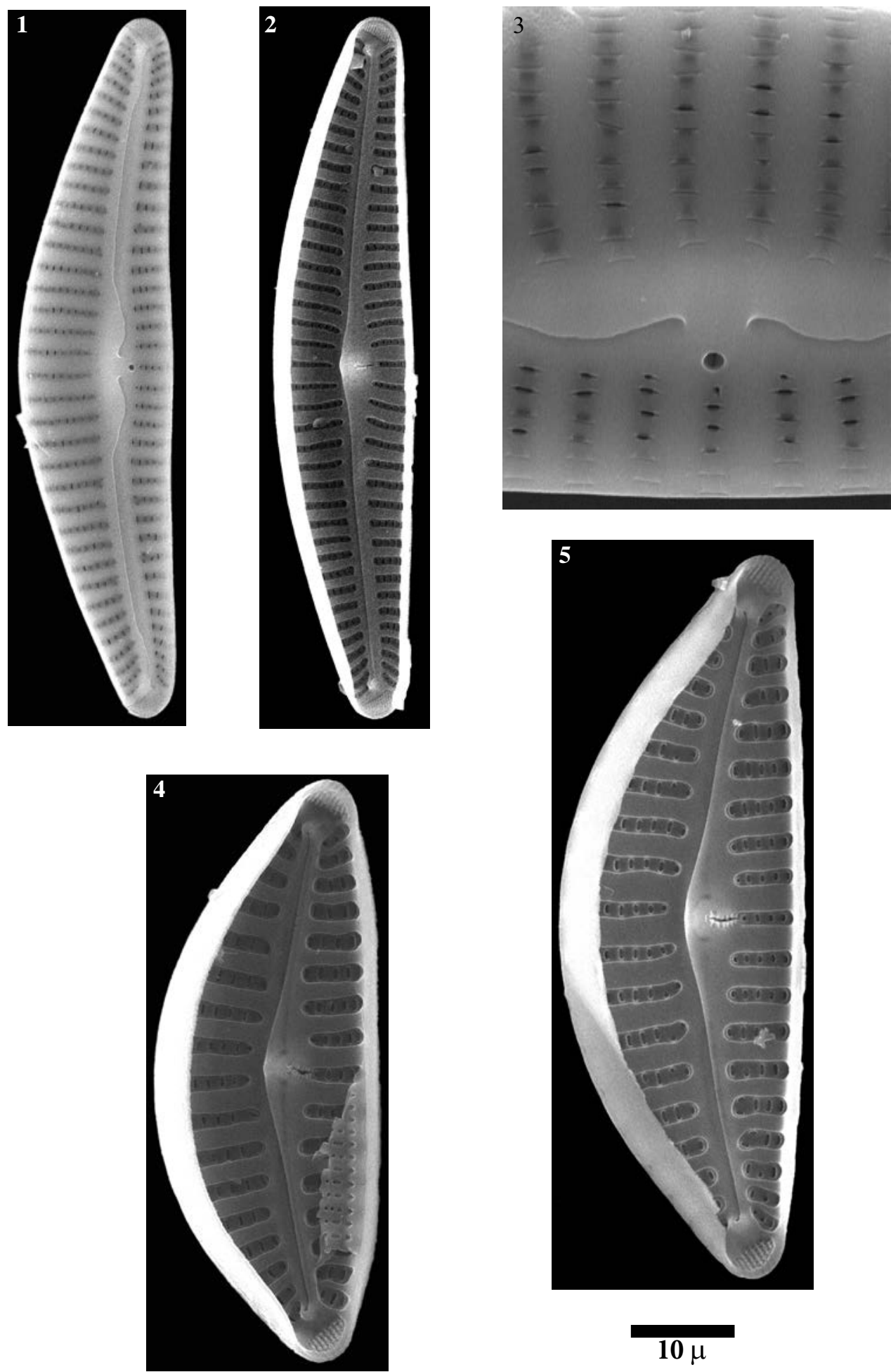
Plate $95 \quad$ LM: $x 1500$

SEM: Figs. 2,5 x2000, Figs. 3-4 x4000, Fig. 6 x5000

\section{Cymbella lange-bertalotii Krammer}

Fig. 1 Lake Arratille, sediment PYR11

Figs. 2-6 Lake Port Bielh, sediment EpiPYR28 

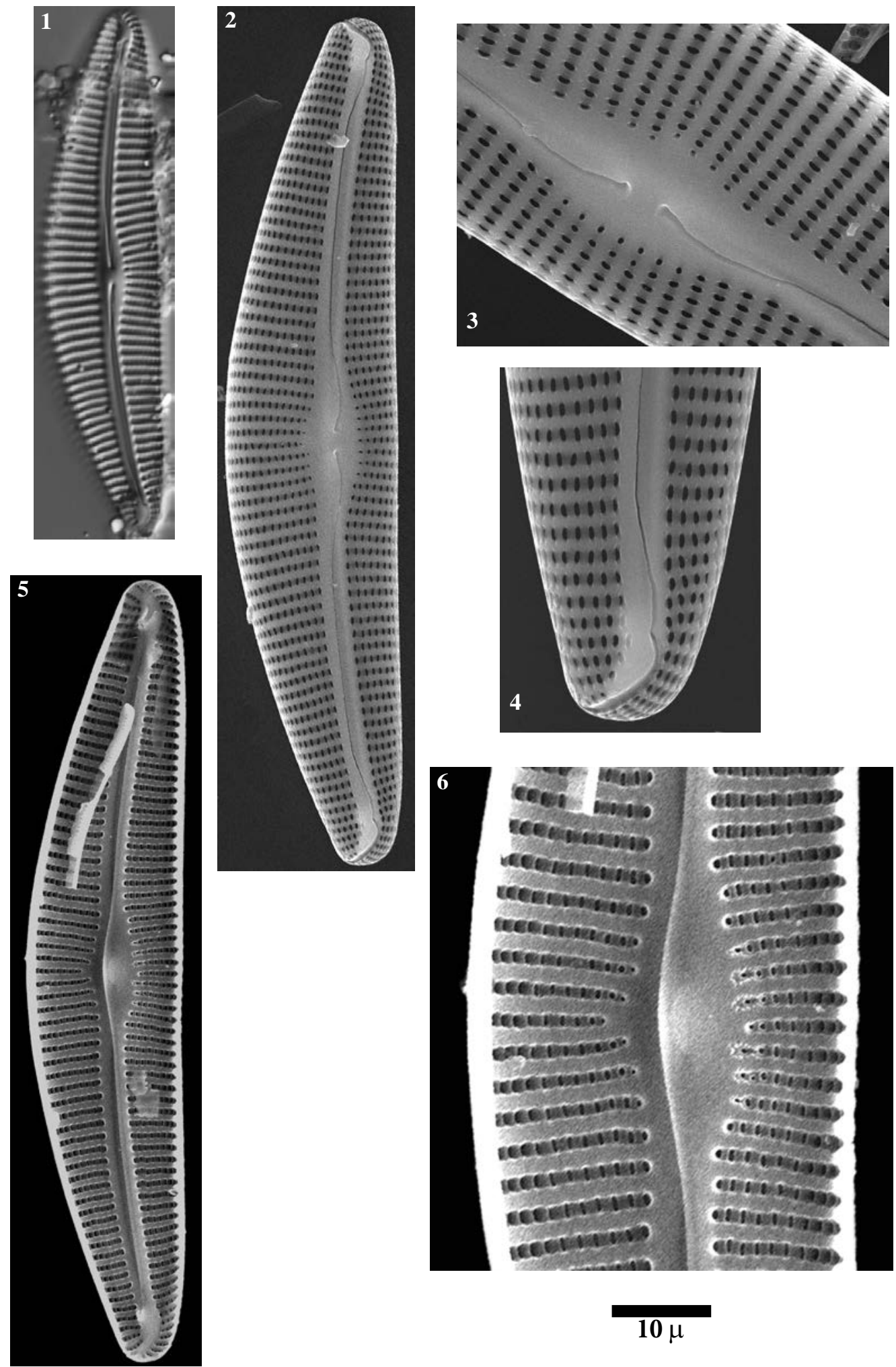

$10 \mu$ 
Plate $96 \quad$ LM: $x 1500$

SEM: Fig. 3,6 x6000, Fig. 5 x1500, Fig. 7 x 10000

Figs. 1-2 Cymbella cf. cymbiformis Agardh

Figs. 3-7 Cymbella cymbiformis Agardh

Fig. 1 Lake Arratille, sediment PYR11

Fig. 2 Lake Sen, sediment PYR40

Figs. 3-7 Lake Roumassot, sediment PYR04

Fig. 4 Lake Posets, sediment PYR42 

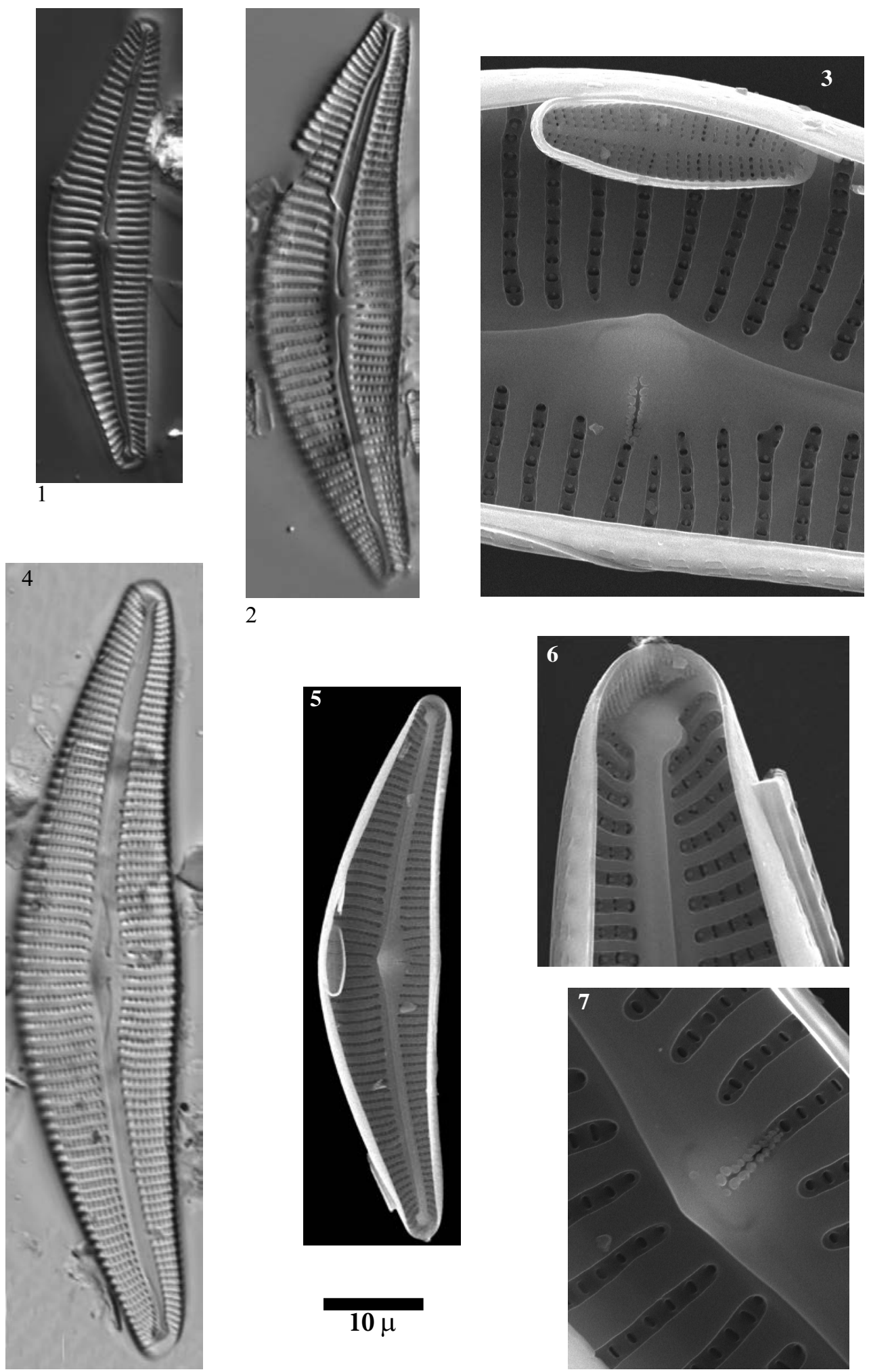

Limnetica, 36 (1): 127-395 (2017) 
Plate $97 \quad$ LM: $x 1500$

SEM: Fig. 2 x1500, Figs. 3-4 x6000

Cymbella cf. cymbiformis Agardh

Fig. 1 Lake Gran de Mainera, sediment PYR70

Figs. 2-4 Lake Burg, sediment BURG 939

Fig. 5 Lake Burg 

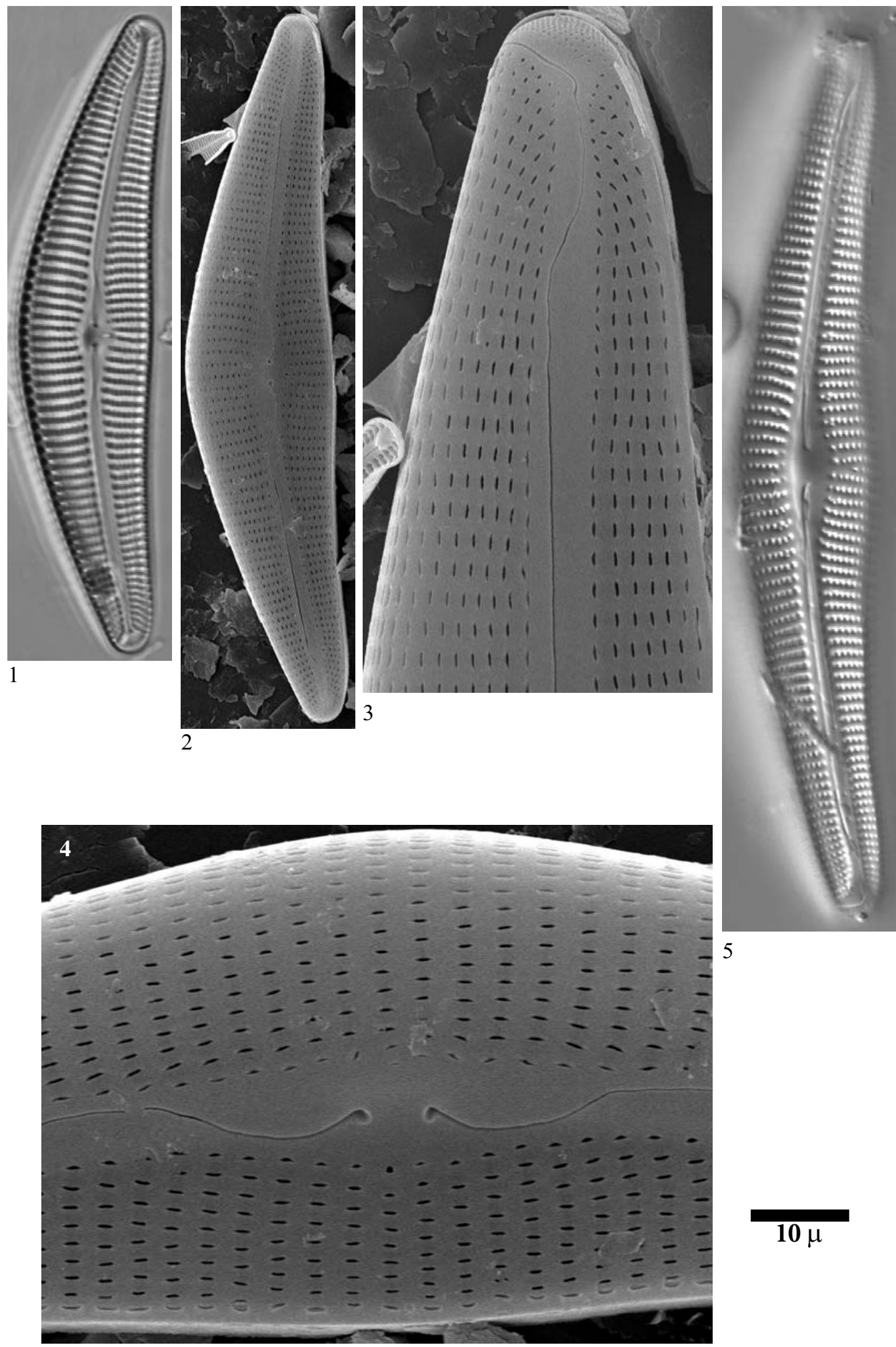
Plate $98 \quad$ LM: $x 1500$

SEM: x6000

Figs. 1-4 Cymbella dorsenotata Østrup

Fig. 1 Lake Arratille, sediment PYR11

Fig. 2 Lake Arnales, sediment PYR09

Figs. 3-4 Lake Roumassot, sediment PYR04 

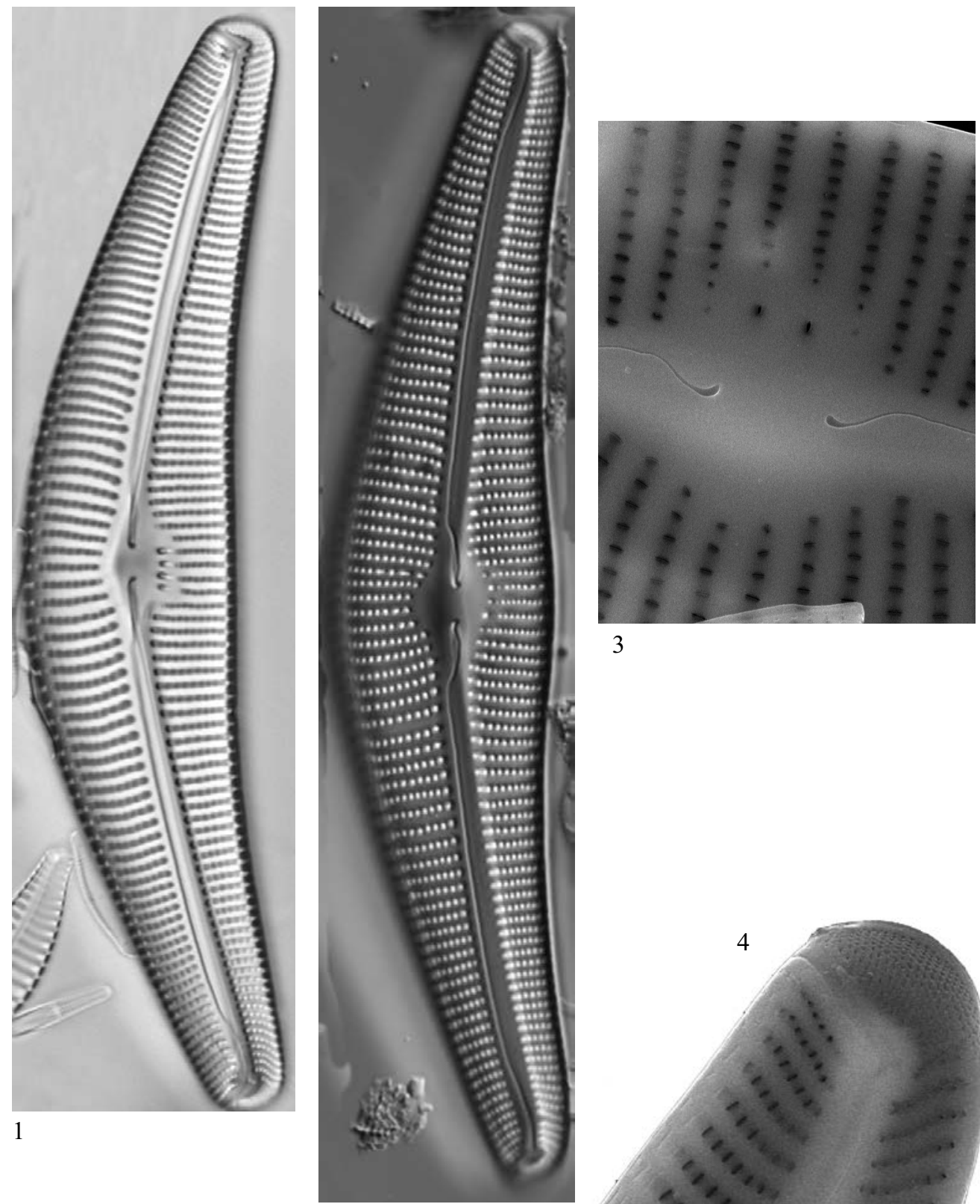

2

$10 \mu$

Limnetica, 36 (1): 127-395 (2017) 
Figs. 1-2

Figs. 3-5

Figs. 6-7

Figs. 8-11

Figs. 12-13

Figs. 1-2

Figs. 3, 5-7

Figs. 4, 9-11, 13

Fig. 8

Fig. 12
Cymbella neoleptoceros var. tenuistriata Krammer

Cymbella cf. neocistula Krammer

Cymbella excisa Kützing

Cymbella cf. subcistula Krammer

Cymbella cf. proxima Reimer

Lake Acherito, sediment PYR01

Lake Posets, sediment PYR42

Lake Gros de Camporrells, sediment PYR110

Lake Angonella de Mes Amunt, sediment PYR78

Lake Arnales, sediment PYR09 

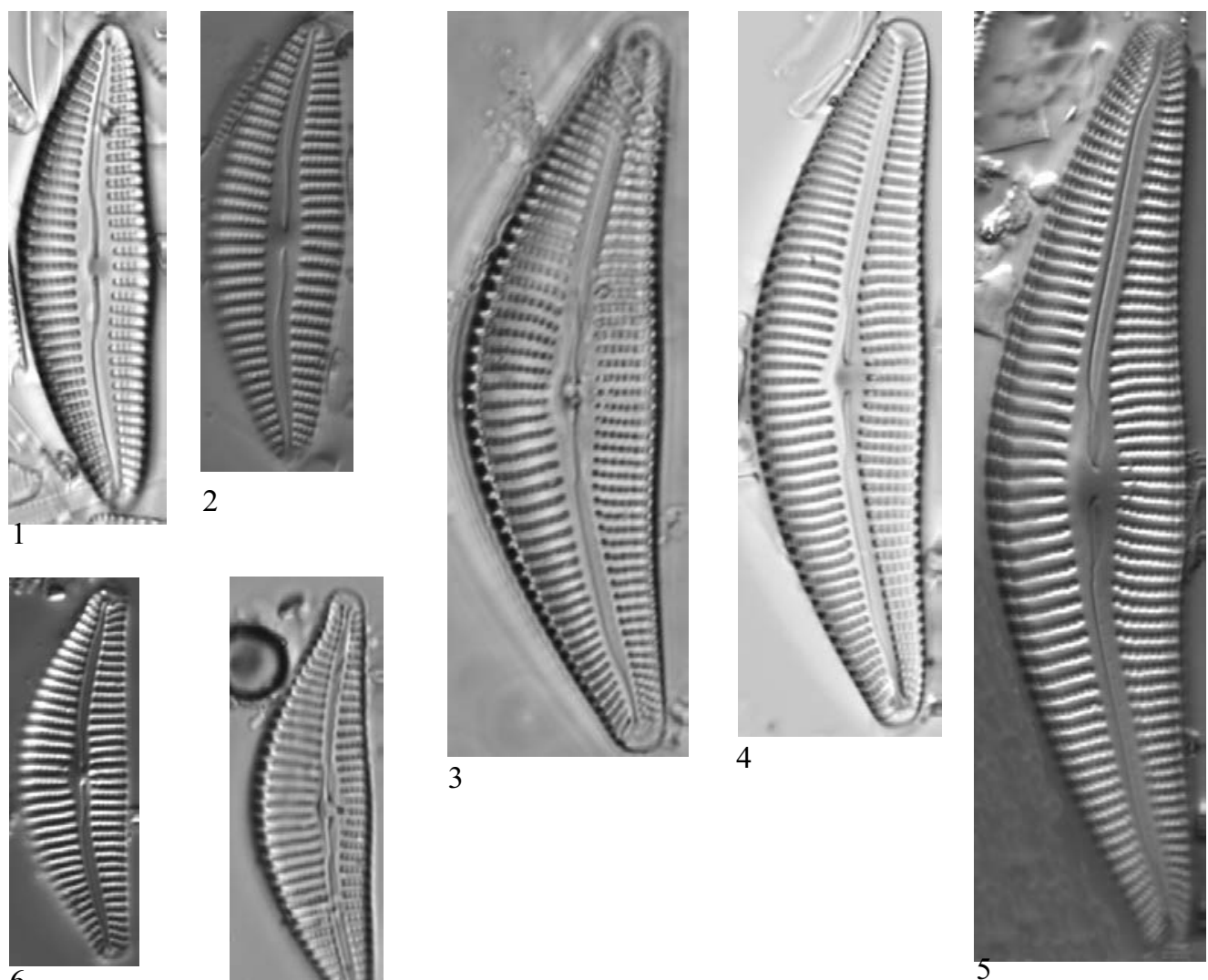

6

2
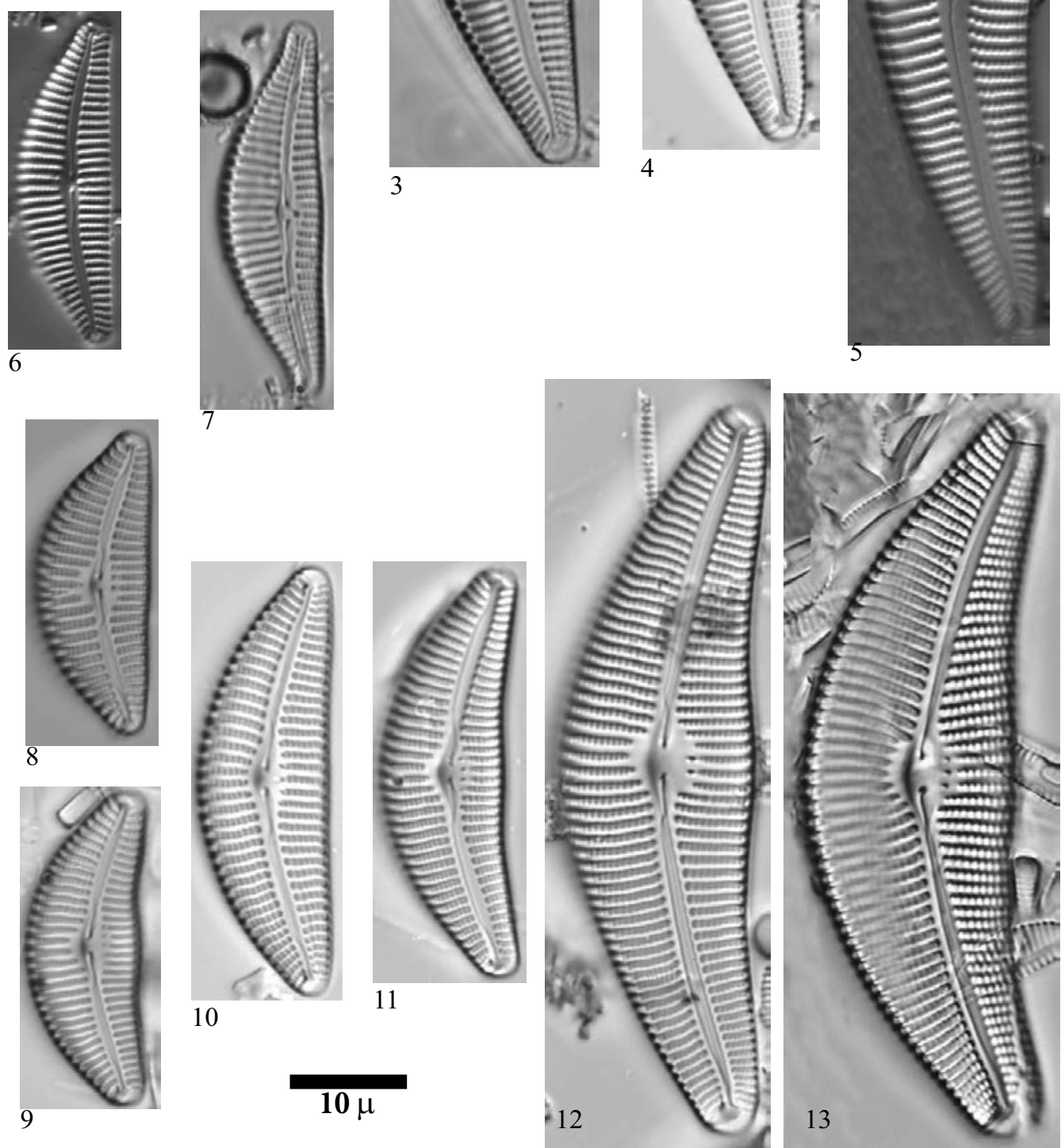

Limnetica, 36 (1): 127-395 (2017) 
Figs. 1-4

Fig. 5

Fig. 6

Figs. 7-8

Fig. 9

Fig. 10

Fig. 11

Figs. 12-13
Cymbopleura acuta var. angusta Krammer

Cymbella subcuspidata Krammer

Cymbopleura apiculata Krammer

Cymbopleura cf. hercynica (Schmidt) Krammer

Cymbopleura sp. No. 2 Burg

Cymbopleura sp

Cymbopleura anglica (Lagerstedt) Krammer

Cymbopleura naviculiformis (Auerswald) Krammer
Fig. 1

Fig. 2

Figs. 3-4

Fig. 5

Fig. 6

Fig. 7

Fig. 8

Fig. 9

Fig. 10

Fig. 11

Fig. 12

Fig. 13
Lake Forcat Inf., sediment PYR77

Lake Bleu de Rabassoles, sediment PYR112

Lake Sotllo, sediment PYR89

Lake Les Laquettes, sediment PYR27

Lake Plan, sediment PYR69

Lake Posets, sediment PYR42

Lake Eriste, sediment PYR43

Lake Burg, sediment BURG 1021

Lake Burg, sediment BURG 833

Lake Arratille, sediment PYR11

Lake Pixón, sediment PYR44

Lake Burg 

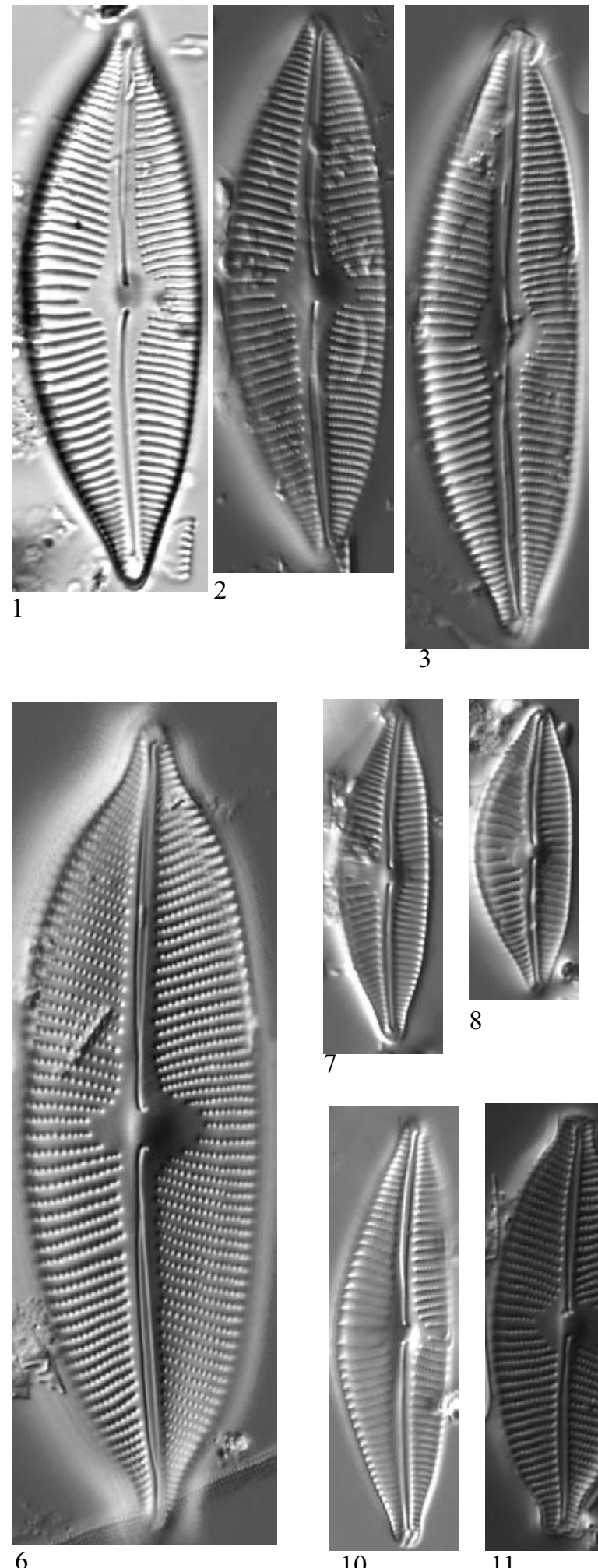
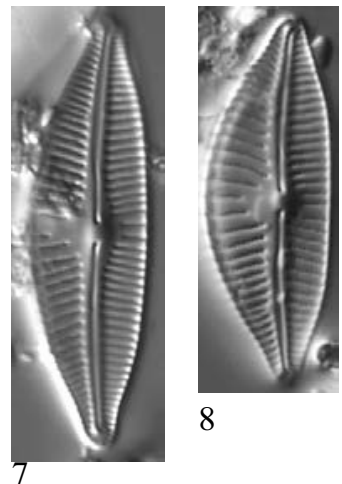

8

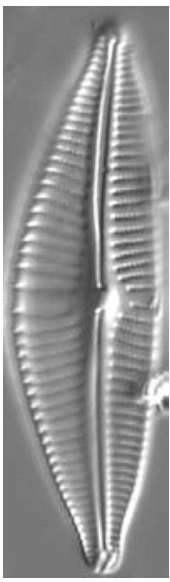

10

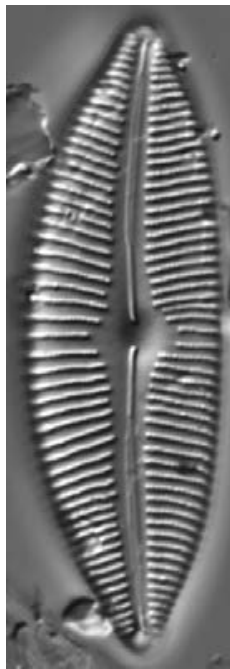

4
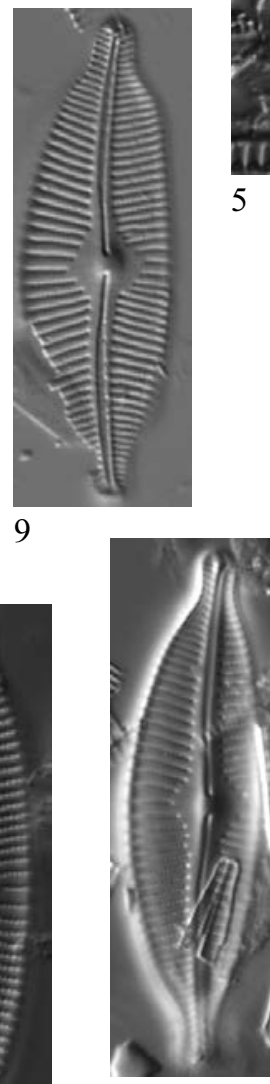

12

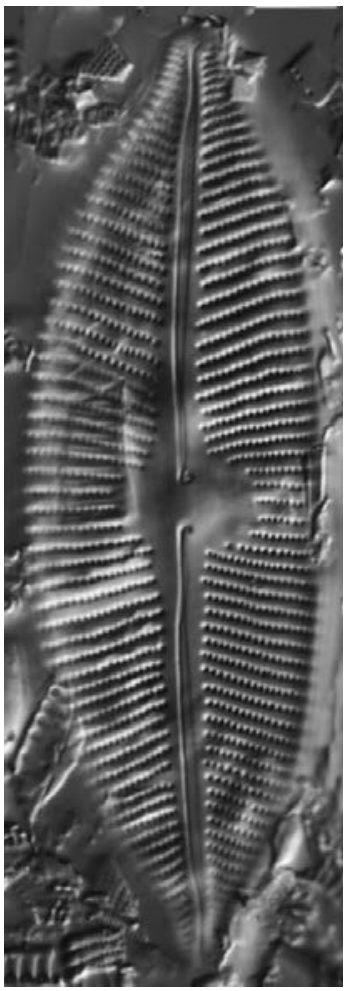

5

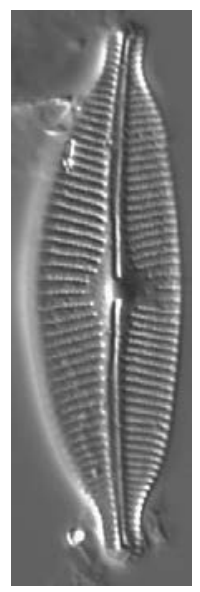

13

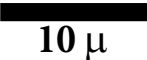


Plate $101 \quad$ LM: x1500

Figs. 1-2 Cymbopleura inaequalis (Ehrenberg) Krammer

Fig. 3 Cymbopleura subaequalis var. truncata Krammer

Fig. $4 \quad$ Cymbopleura subaequalis (Grunow) Krammer

Figs. 5-6 Cymbopleura cf. subaequalis (Grunow) Krammer

Figs. 1, $4 \quad$ Lake Arratille, sediment PYR11

Fig. 2 Lake Ormiélas, sediment PYR05

Fig. 3 Lake Urdiceto, sediment PYR125

Figs. 5-6 Lake Monges, sediment PYR57 

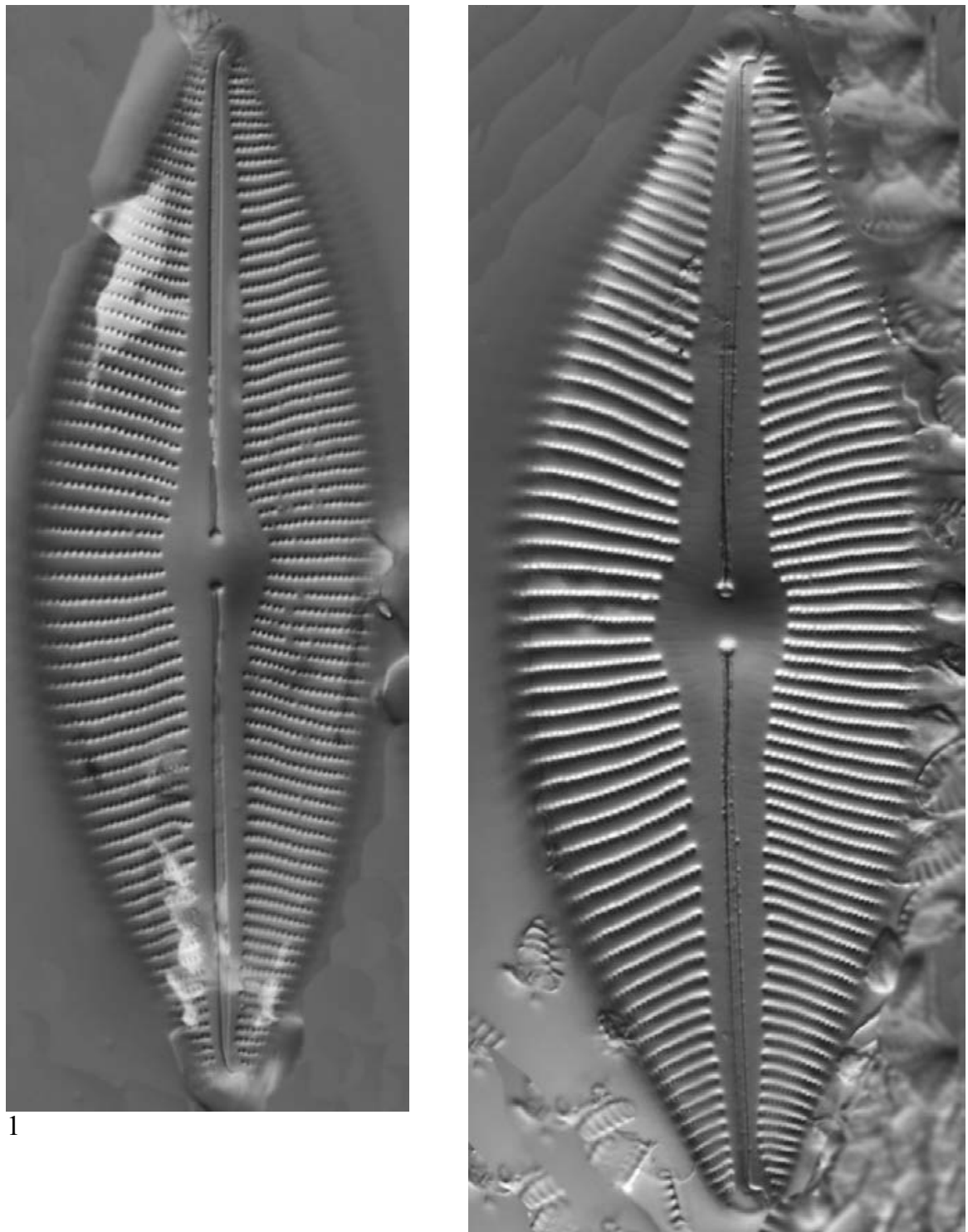

2
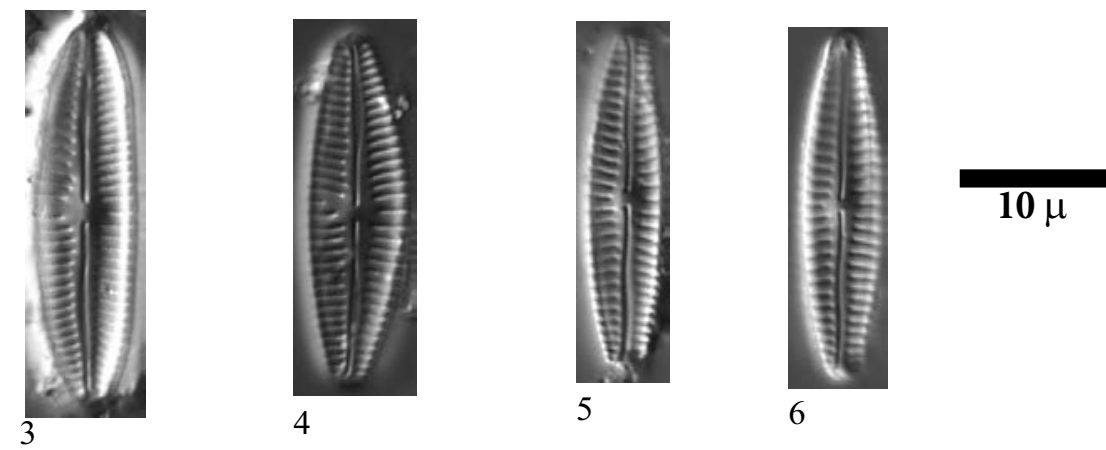

Limnetica, 36 (1): 127-395 (2017) 
Figs. 1-4

Fig. 5

Figs. 6-8

Fig. 9

Figs. 10-16

Figs. 17-23
Delicata delicatula (Kützing) Krammer

Cymbella sp cf. lancettula (Krammer) Krammer

Cymbopleura cf. pyrenaica Le Cohu \& Lange-Bertalot Cymbella sp.

Encyonopsis aequalis (Smith) Krammer

Encyonopsis aff. aequalis (Smith) Krammer

Encyonopsis aff. kriegeri (Krasske) Krammer

Fig. 1

Fig. 2

Figs. 3-4

Fig. 5

Fig. 6

Figs. 7-8

Figs. 9, 19-20

Figs. 10-11, 13-18, 21-23

Fig. 12
Lake Arratille, sediment PYR11

Lake Posets, sediment PYR42

Lake Gran de Mainera, epilithic EpiPYR70

Lake Bachimala Sup., sediment PYR31

Lake Estom, sediment PYR15

Lake Rond, sediment PYR72

Lake Senó, sediment PYR84

Lake Sotllo, sediment PYR89

Lake Negre, sediment PYR79 

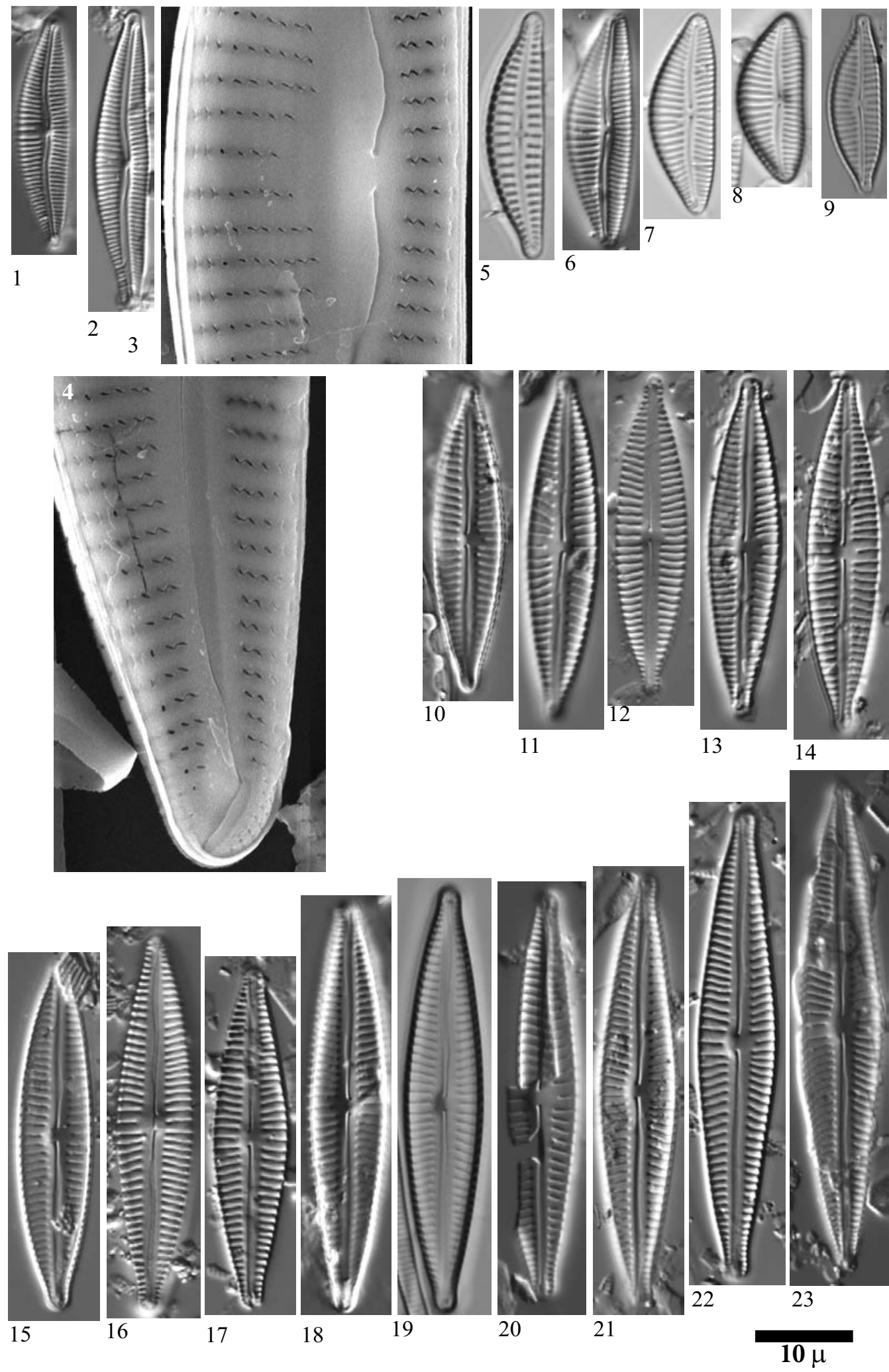

Limnetica, 36 (1): 127-395 (2017) 
SEM: Fig. 16 x10000, Figs. $17-19 \times 5000$

Figs. 1-2

Figs. 3-5

Figs. 6-7

Fig. 8

Fig. 9

Figs. 10-19

Figs. 20-22

Figs. 23-24

Figs. 25-26

Figs. 27-34

Fig. 1

Fig. 2

Figs. 3-5, 8, 10, 19,23

Figs. 6-7

Fig. 9

Fig. 11

Figs. 12-13

Fig. 14

Fig. 15

Fig. 20

Figs. 21-22, 27-34

Fig. 24

Fig. 25

Fig. 26
Encyonopsis grunowii Krammer

Encyonopsis cesatii (Rabenhorst) Krammer

Encyonopsis cf. falaisensis (Grunow) Krammer

Encyonopsis descripta (Hustedt) Krammer

Encyonopsis cf. lanceola (Grunow) Krammer

Encyonopsis subminuta Krammer \& Reichardt

Encyonopsis microcephala (Grunow) Krammer

Encyonopsis minuta Krammer et Reichardt

Encyonopsis sp. No.1 Nere

Encyonopsis cf. krammeri Reichardt

Lake Llosás, sediment PYR46

Lake Senó, sediment PYR84

Lake Posets, sediment PYR42

Lake Basa de la Mora, sediment PYR32

Lake Filià, sediment PYR71

Lake Bersau, sediment PYR03

Lake Burg, sediment BURG 831

Lake Arretille, sediment PYR11

Lake Burg, sediment BURG 1127

Lake Helado de Marboré, sediment PYR18

Lake Acherito, epilithic EpiPYR01

Lake Col d'Arratille, sediment PYR12

Lake Col d'Arratille, epilithic EpiPYR12

Lake Nere de Güèri, epilithic EpiPYR53 

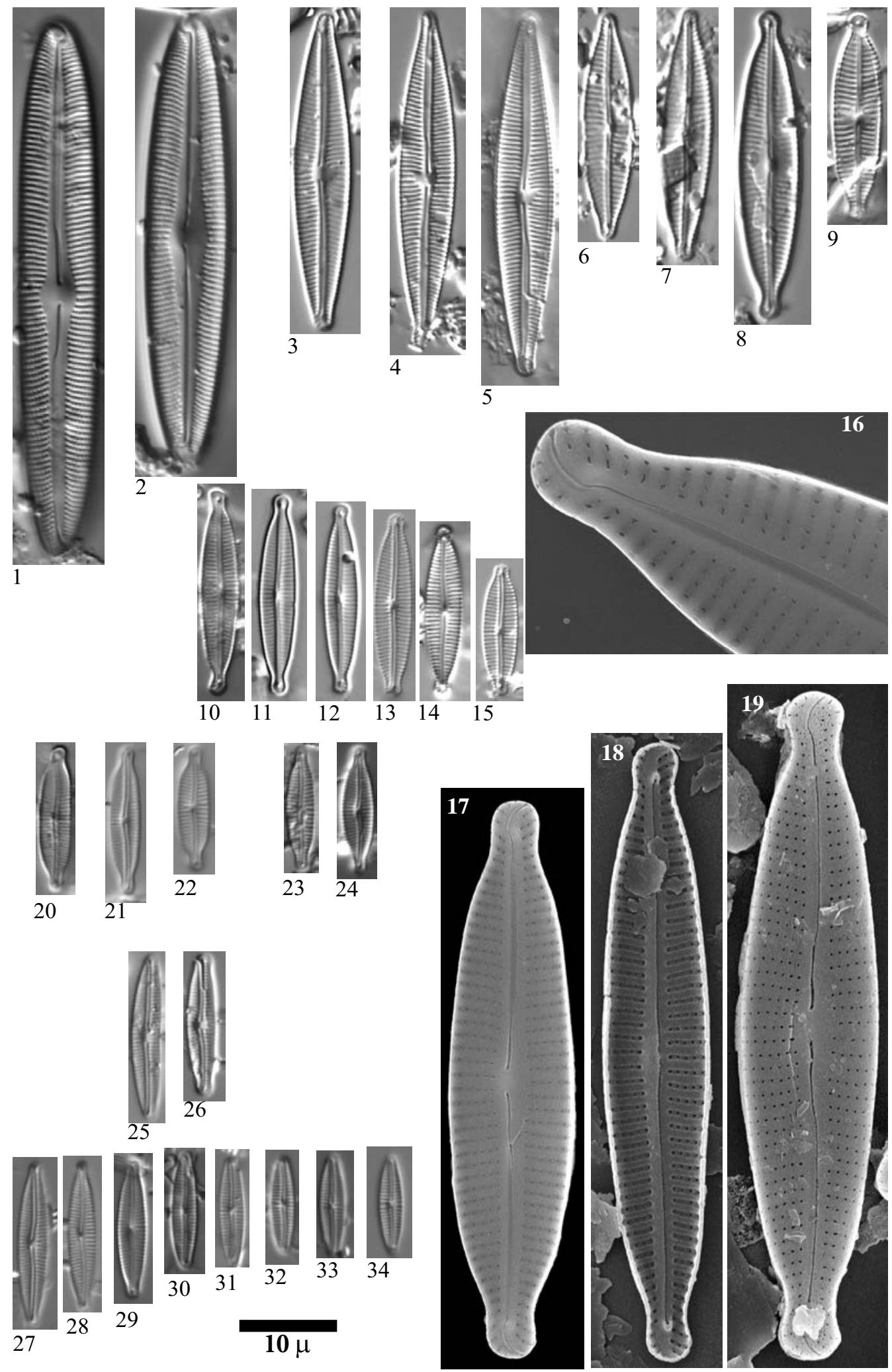

Limnetica, 36 (1): 127-395 (2017) 
Plate $104 \quad$ LM: x1500

SEM Figs 11,15 x4000, Fig. 16 x10000

Figs. 1-2 Encyonema vulgare Krammer

Figs. 3-16 Encyonema silesiacum (Bleisch) Mann

Figs. 17-19 Encyonema lange-bertalotii Krammer

Figs. 1-2 Lake Angonella, sediment PYR78

Figs. 3-5, 10, 19 Lake La Munia Sup., sediment PYR20

Figs. 6, $12 \quad$ Lake Arratille, sediment PYR11

Fig. $7 \quad$ Lake Sen, sediment PYR40

Figs. 8-9, 13-14 Lake Posets, sediment PYR42

Fig. $17 \quad$ Lake Arnales, sediment PYR09

Fig. 18 Lake Rond, sediment PYR72

Fig. 11, 15-16 Lake Port Bielh, epilithic EpiPYR28 

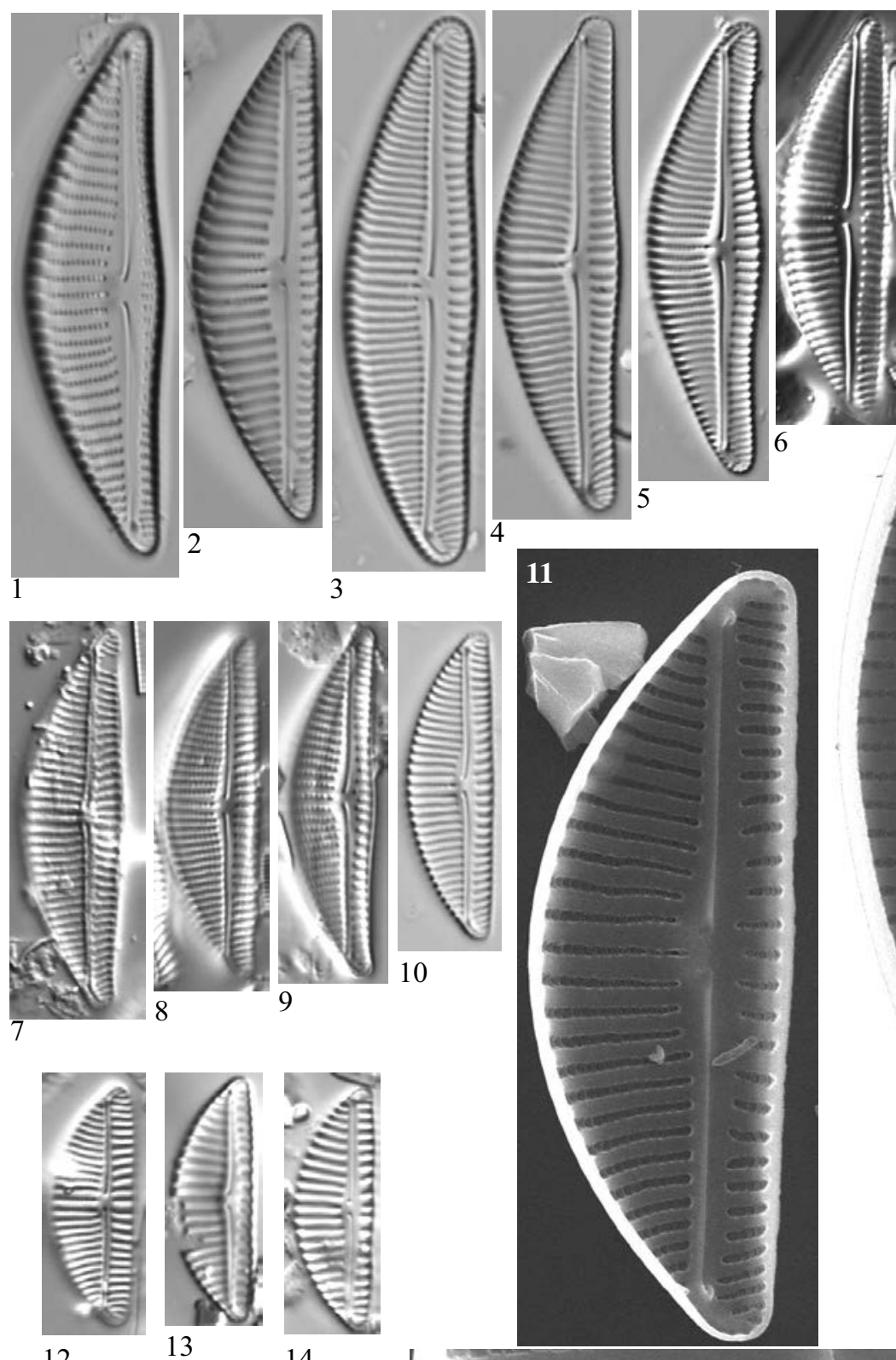

12
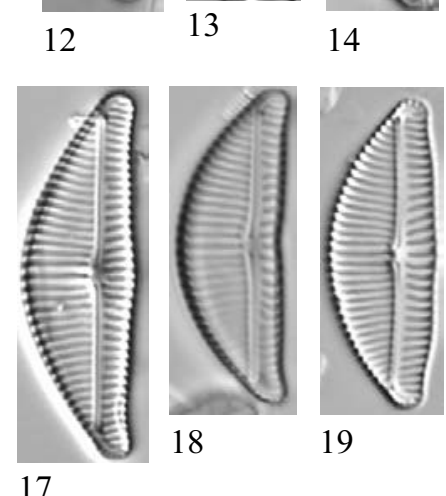

19
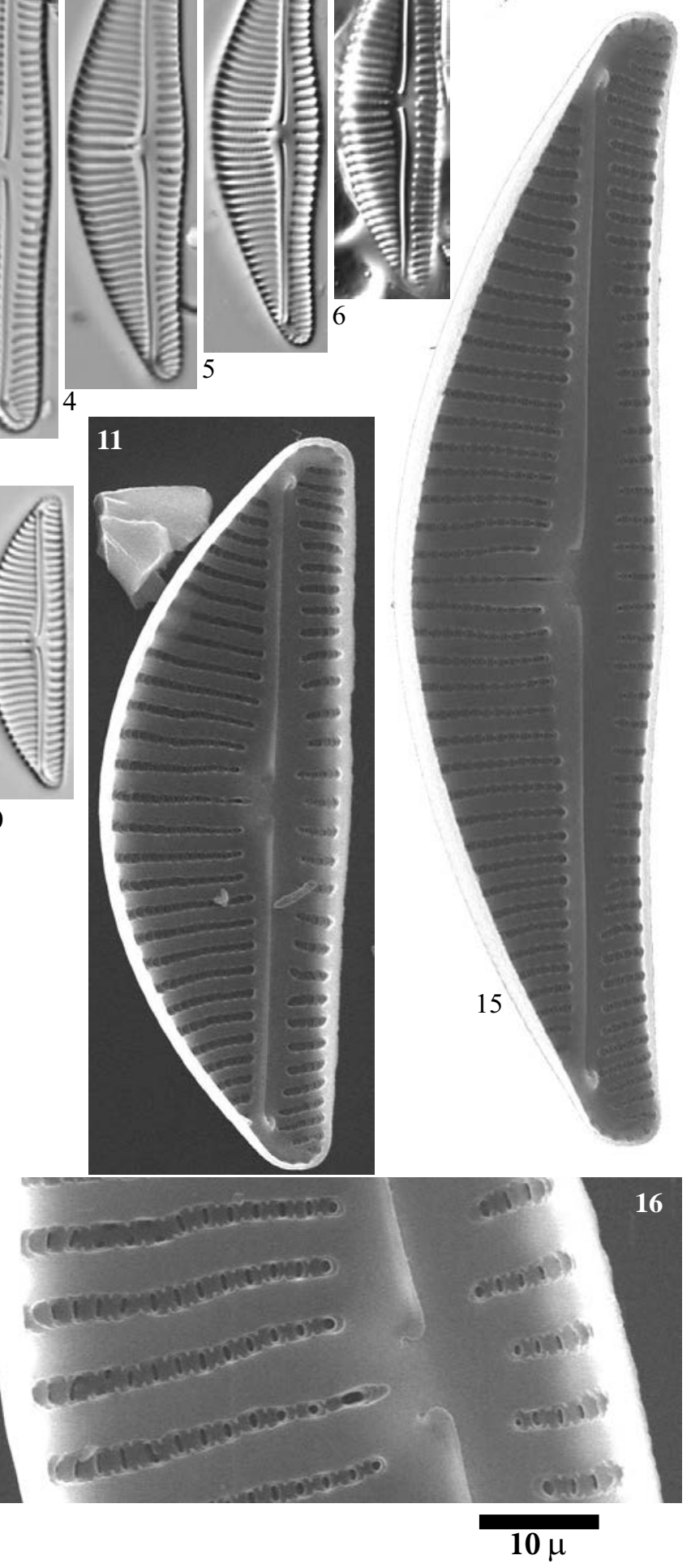

Limnetica, 36 (1): 127-395 (2017) 
LM: $\mathrm{x} 1500$

SEM: Figs. 45,46,48 x6000, Fig. 47 ×10000

Fig. 1

Figs. 2-7

Figs. 8-10

Fig. 11

Fig. 12

Fig. 13

Figs. 14-31,45

Figs. $35-40$

Fig. 41

Figs. 42-44,48

Figs. 46-47

Fig. 1

Figs. 2-4, 7, 14

Fig. 5

Fig. 6

Fig. 8

Fig. 9

Fig. 10

Fig. 11

Figs. 12, 18-19, 22

$$
25,27
$$

Figs. 13, 15-16, 20-21, 23, 26, 35-36

Fig. 24

Fig. 29

Fig. 30

Figs. 17, 28, 31

Figs. 32-34

Figs. 37-39
Encyonema sp. No. 9 Gerber

Encyonema sp. No. 10 Burg, aff. minutum (Hilse) Mann

Encyonema sp. No. 1 Mora

Encyonema sp. No. 8 Filia

Encyonema sp. No. 2 Sen

Encyonema sp.

complex

Encyonema minutum (Hilse) Mann

Encyonema ventricosum (Kützing) Grunow

Encyonema sp.

Encyonema ventricosum (Kützing) Grunow

Encyonema sp. No. 7 Barroude

Encyonema reichardtii (Krammer) Mann

Encyonema minutum (Hilse) Mann

Lake Gerber, sediment PYR63

Fig. 40

Lake Eriste, sediment PYR43

Lake Burg

Fig. 41

L. Barroude Inf., sediment PYR29

Lake Burg, sediment BURG 831

Figs. $42-43$

Lake Cap Long, sediment PYR24

L. Burg, sediment BURG 1007

Fig. 18

Lake Pixón, sediment PYR44

L. Col d'Arratille, sed. PYR12

Fig. 45

L. Roumassot, sediment EpiPYR04

L. Basa de la Mora, sed. PYR32

Figs. 46-47

Lake Roumassot, sediment PYR04

Lake Glacé, sediment PYR17

Fig. 48

L. Pondiellos Sup., sed. EpiPYR08

Lake Filià, sediment PYR71

Lake Sen, sediment PYR40

Lake Posets, sediment PYR42

Lake Bersau, sediment PYR03

L. H. Monte Perdido, sed. PYR19

L. Bleu de Rabass., sed. PYR112

L. Arratille, sediment PYR11

L. Ormiélas, sediment PYR05

L. Angonella, sediment PYR58 

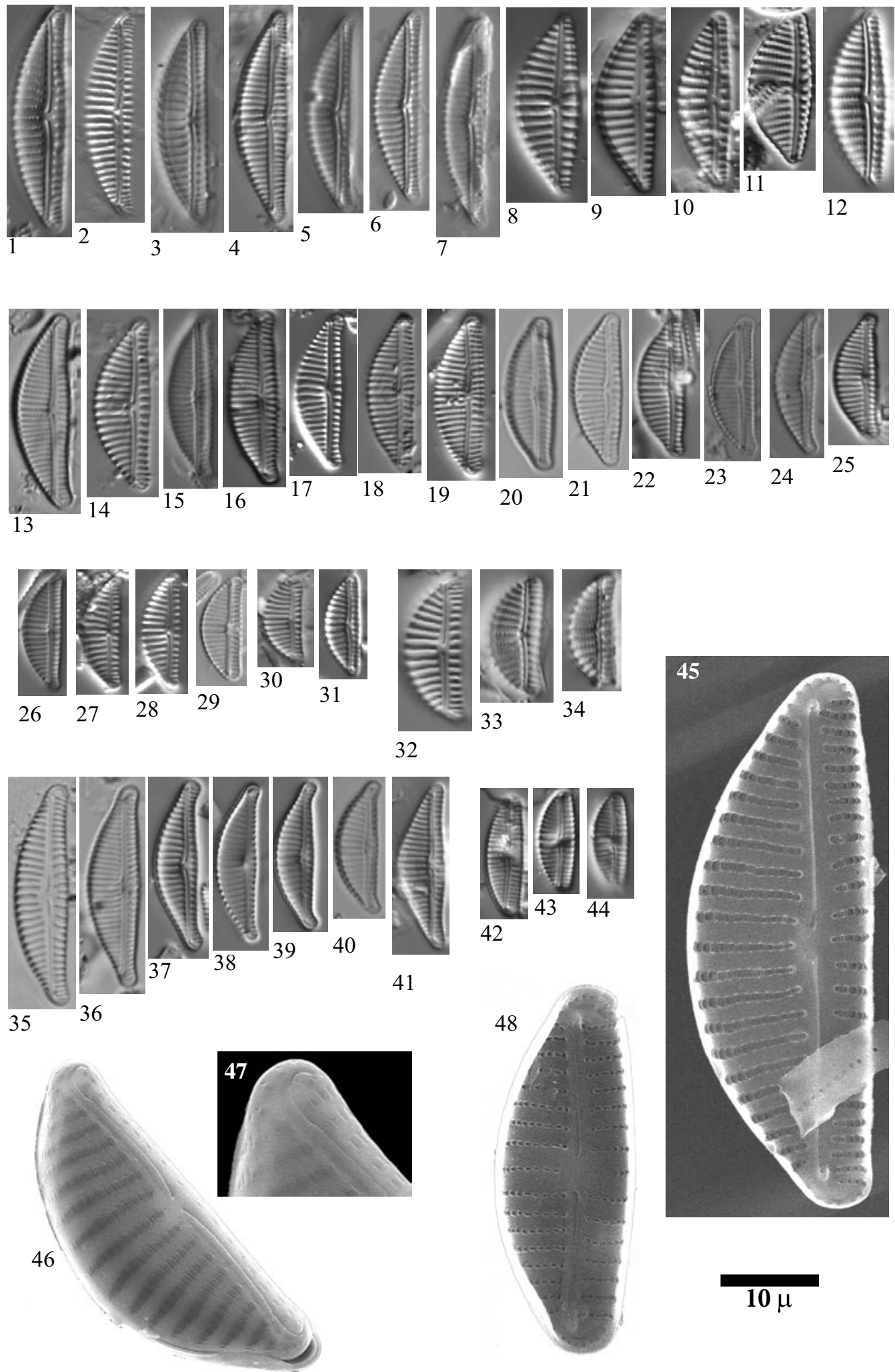

Limnetica, 36 (1): 127-395 (2017) 
Plate $106 \quad$ LM: x1500

SEM: Figs. 1, 3, 4 x10000, Figs. 5,6 x4000, Fig. 7 x2000

Figs. 1-2 Encyonema minutum (Hilse) Mann

Figs. 3-7 Encyonema ventricosum (Kützing) Grunow

Figs. 1-2 Lake Laurenti, sediment PYR111

Figs. 4-5 Lake Redon, sediment REDOM

Figs. 6-7 Lake Pondiellos, epilithic EpiPYR08

Fig. 3 Lake Posets, sediment PYR42 

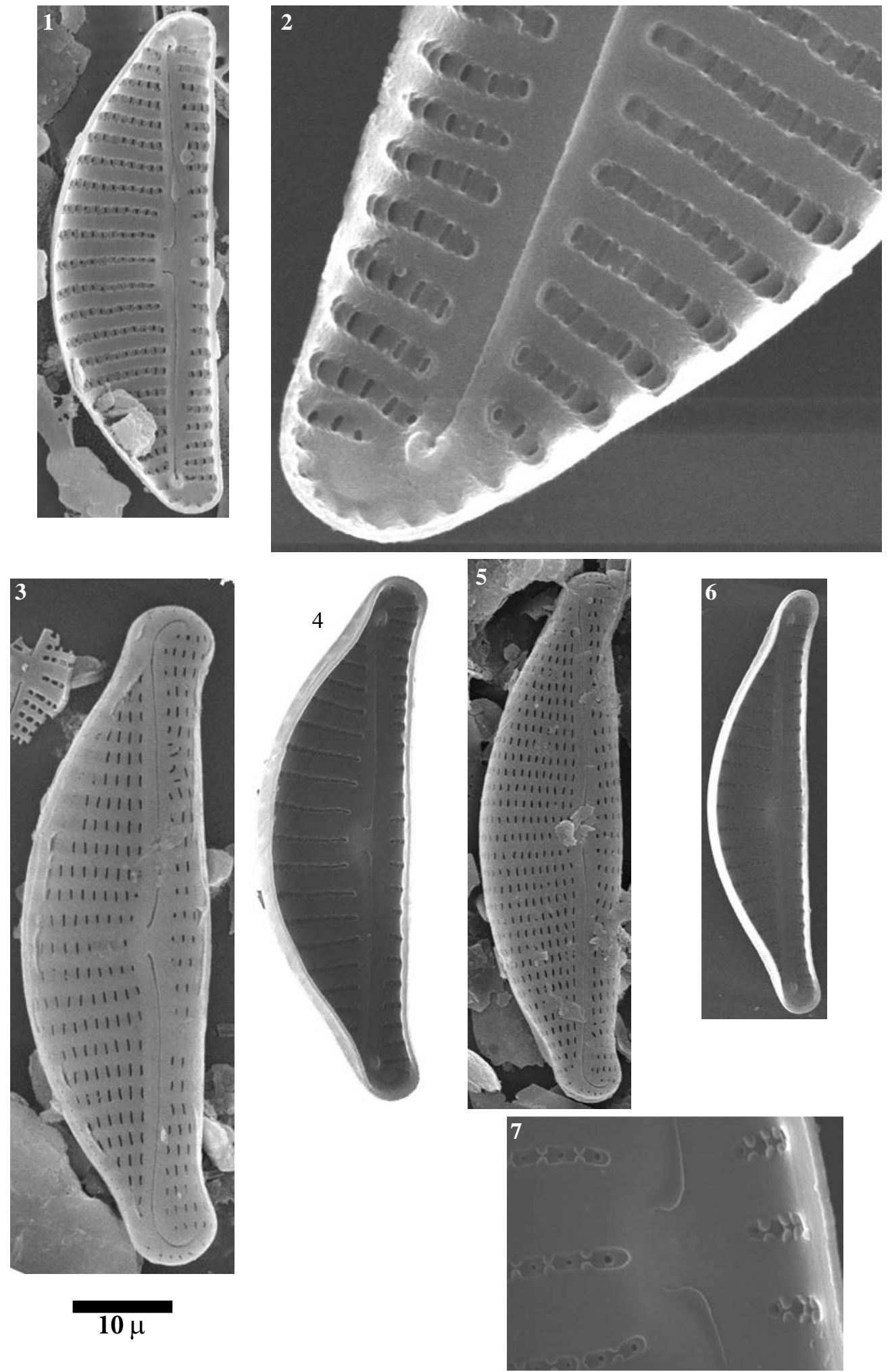

Limnetica, 36 (1): 127-395 (2017) 
Plate $107 \quad$ LM: $x 1500$

SEM: Figs. 14-16 x6000, Figs. 17-18 x4000

Figs. 1-4, 14

Encyonema perpusillum (Cleve) Mann

Figs. 5-9,

Encyonema gaeumannii (Meister) Krammer

15-16

Figs. 10-13

Encyonema neogracile Krammer

$17-18$

Figs. 1-2

Figs. 3-4

Figs. 5-7

Fig. 8

Fig. 9

Figs. 10-13

Fig. 14

Fig. 15

Figs. 16-18
Lake Aubé, sediment PYR82

Lake Monges, sediment PYR57

Lake Blaou, sediment PYR94

Lake Posets, sediment PYR42

Lake Les Laquettes, sediment PYR27

Lake Bleu de Rabassoles, epilithic EpiPYR112

Lake Illa, sediment PYR66

Lake Mariola, sediment PYR80

Lake Redon, sediment REDOM 

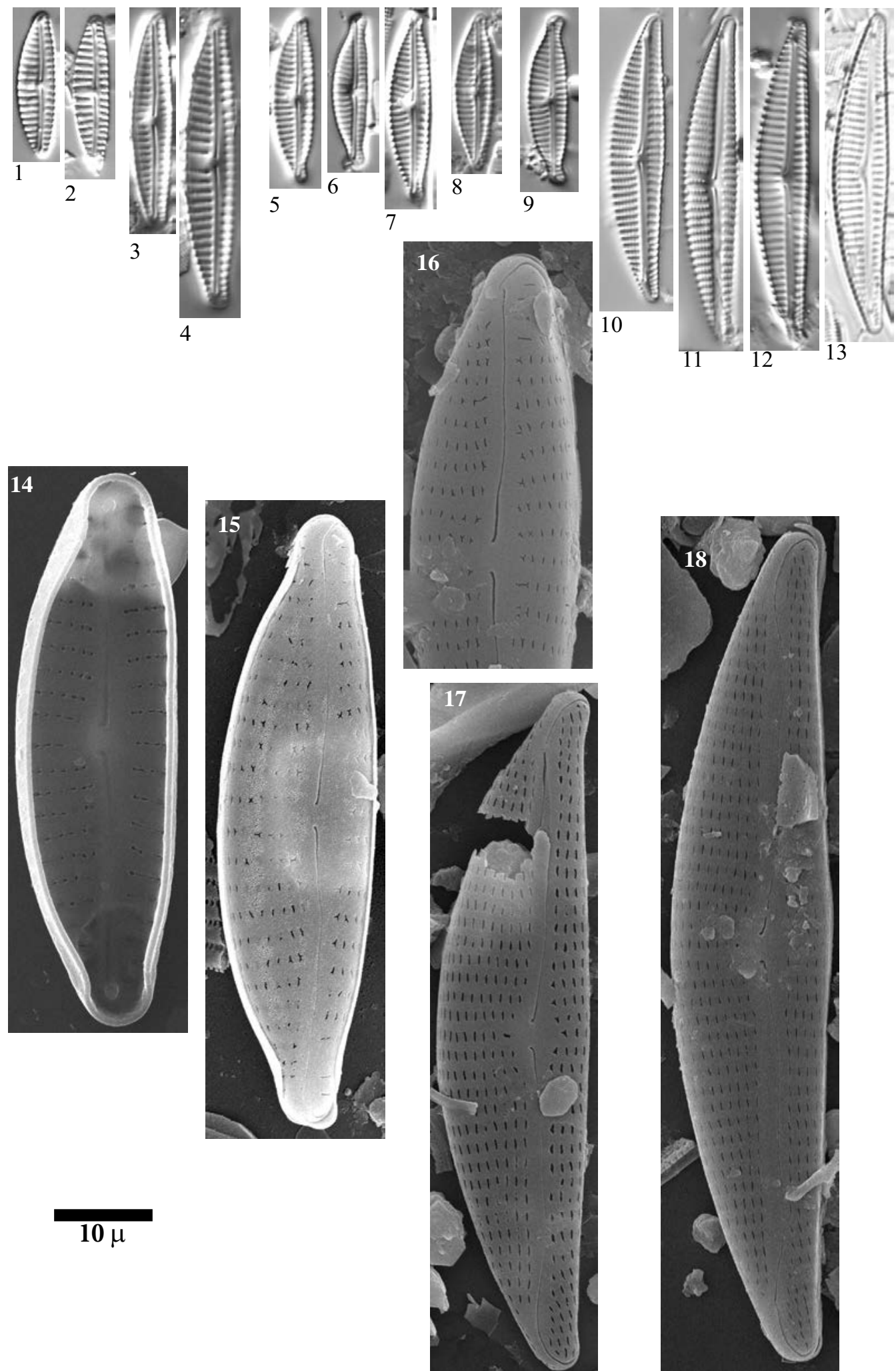

Limnetica, 36 (1): 127-395 (2017) 
Plate $108 \quad$ LM: $x 1500$

SEM: $\mathrm{x} 3000$

Figs. 1-3 Encyonema caespitosum Kützing

Figs. 4-6 Encyonema cf. obscurum var. alpina Krammer

Fig. 7 Encyonema sp. No. 3 Sen

Fig. 8 Encyonema silesiacum (Bleisch) Mann

Figs. 9-10 Primary cells?

Figs. 11-14 Encyonema sp. No. 5 Pica Palomera

Figs. 15-16 Encyonema sp. No. 6 Seno

Figs. 17-22 Encyonema hebridicum Grunow ex Cleve

Figs. 23-27 Reimeria sinuata (Gregory) Kociolek \& Stoermer emend Sala, Guerrero \& Ferrario

Figs. 1-3, 27 Lake Estom, sediment PYR15

Figs. 4-5, 7 Lake Basa de la Mora, sediment PYR32

Fig. $6 \quad$ Lake Arnales, sediment PYR09

Figs. 8, 21-22 Lake Arratile, sediment PYR76

Figs. 9-10 Lake Posets, sediment PYR11

Figs. 11, 13 Lake Pica Palomera, sediment PYR52

Fig. 12 Lake Mes Amunt de Tristaina, sediment PYR86

Fig. $14 \quad$ Lake Burg

Fig. 15 Lake Senó, sediment PYR84

Fig. 16 Lake La Munia Sup., sediment PYR20

Fig. 17 Lake Mariola, sediment PYR80

Figs. 18, 20 Lake Negre, sediment PYR79

Fig. 19 Lake Monges, sediment PYR57

Fig. $23 \quad$ Lake Laurenti, sediment PYR111

Fig. $24 \quad$ Lake Les Laquettes, sediment PYR27

Fig. 25 Lake Llebreta, sediment PYR58

Fig. 26 Lake Helado del Monte perdido, epilithic EpiPYR19 

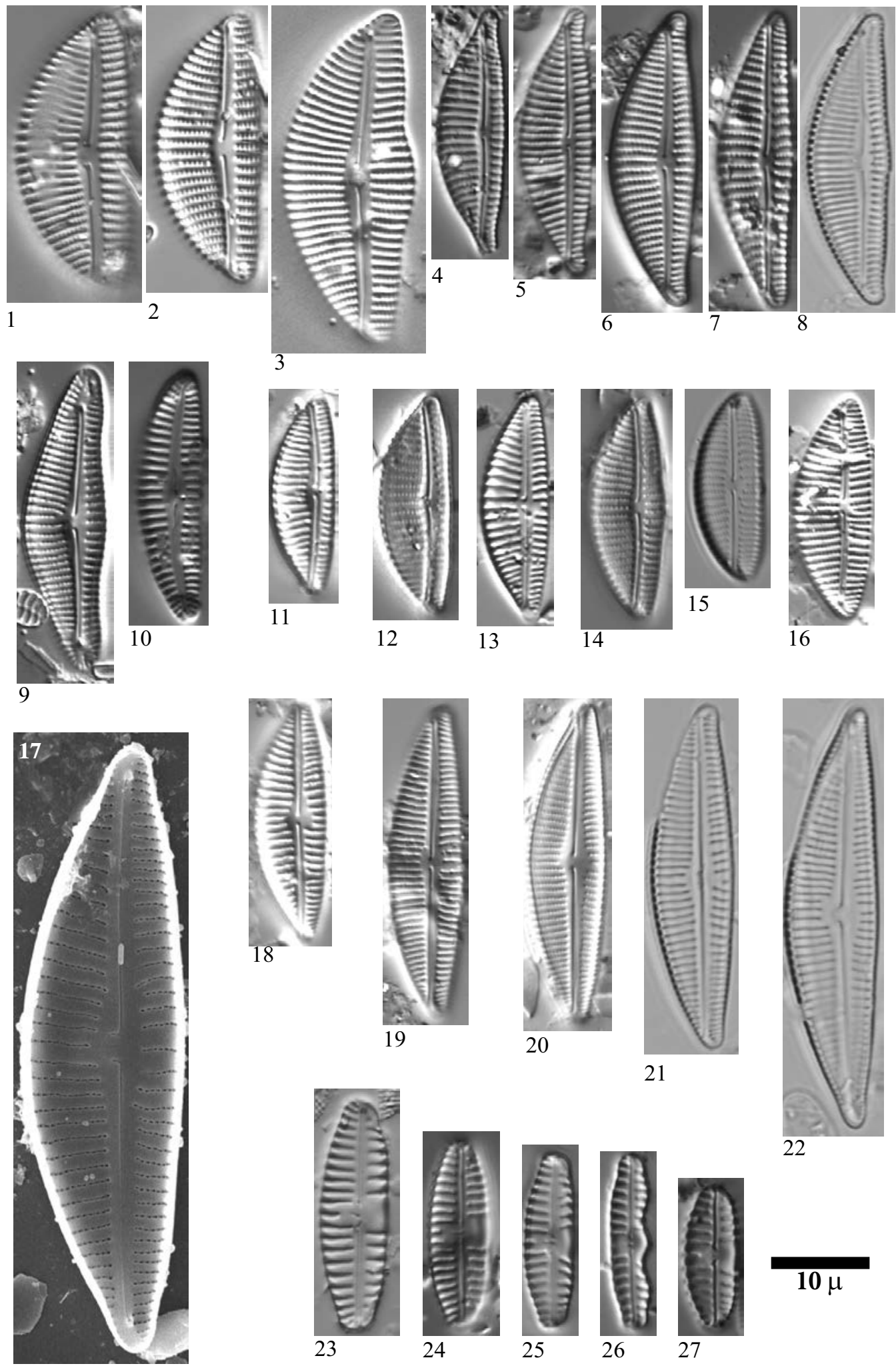
LM: $x 1500$

SEM: Fig. 14 x8000, Fig. 15 x6000, Figs. 22-24 x3000

Figs. 1-2, 14

Figs. 3-4

Figs. 5,15

Fig. 6

Figs. 7-11

Figs. 12-13

Fig. 16

Fig. 17, 22

Figs. 18-20, 23-24

Fig. 21

Fig. 1

Fig. 2

Figs. 3, 7, 8, 9, 13, 16

Figs. 4, 10-12, 19, 21

Figs. 5-6

Fig. 17

Fig. 14-15, 22, 24

Fig. 18

Fig. 20

Fig. 23
Amphora pediculus (Kützing) Grunow

Amphora neglecta f. densestriata Foged

Amphora cf. inariensis Krammer

Amphora sp.

Amphora cf. eximia Carter

Amphora sp. No. 1 Sen

Amphora oligotraphenta Lange-Bertalot

Amphora cf. affinis Kützing

Amphora copulata (Kützing) Schoeman \& Archibald

Amphora lange-bertalotii Z. Levkov \& D. Metzeltin

Lake Llebreta, sediment PYR58

Lake Estanés, sediment PYR02

Lake Sen, sediment PYR40

Lake Posets, sediment PYR42

Lake Pondiellos Sup., sediment PYR08

Lake Basa de la Mora, sediment PYR32

Lake Laurenti, sediment PYR111

Lake Arratille, sediment PYR11

Lake Pixón, sediment PYR44

Lake Arnales, epilithic EpiPYR09 

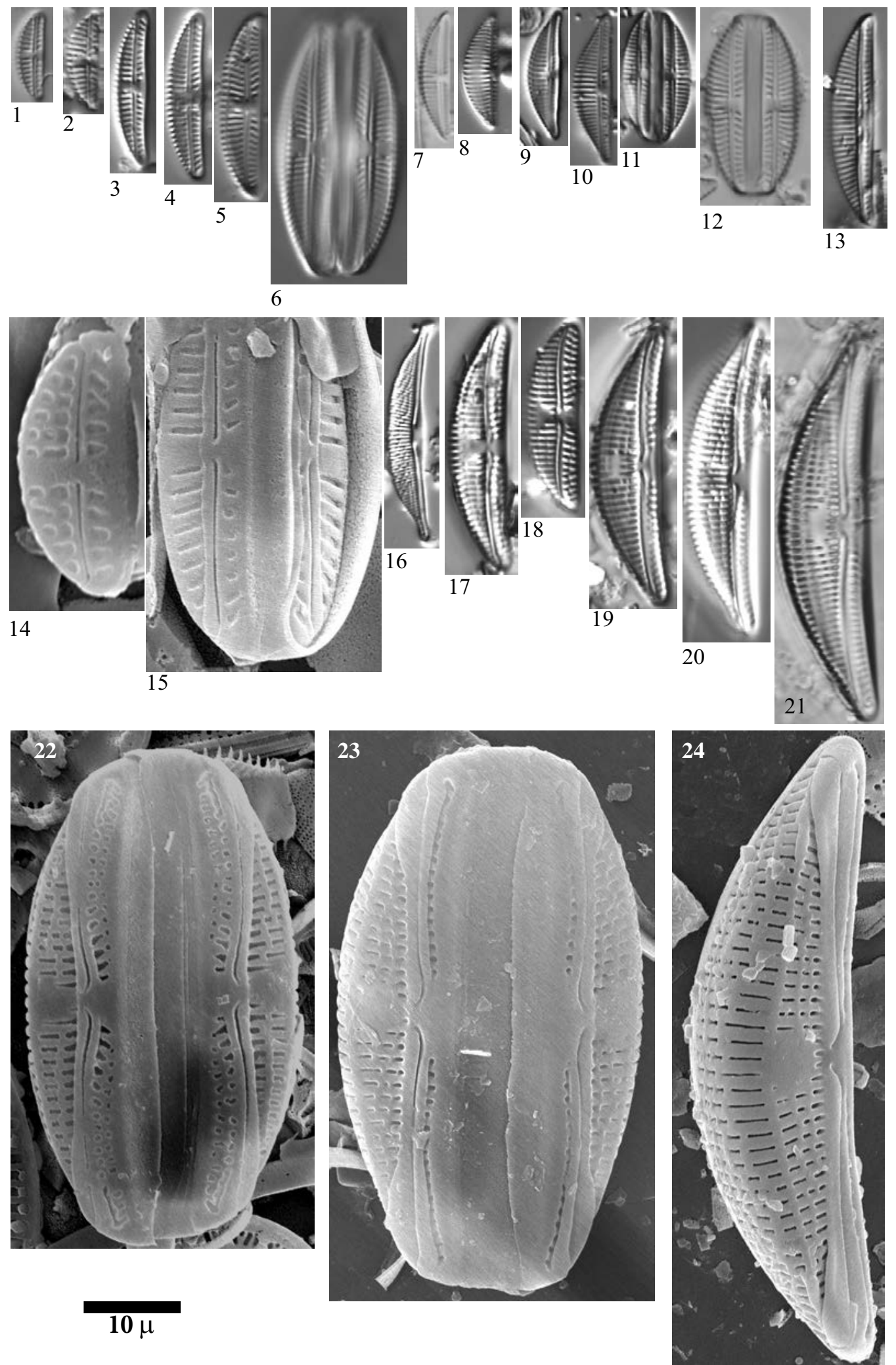

Limnetica, 36 (1): 127-395 (2017) 
Plate $110 \quad$ LM: x1500

SEM: Fig. 10 x10000 Fig. 11 x2000

Fig. 1 Gomphonema coronatum Ehrenberg

Fig. 2 Gomphonema cf. acuminatum Ehrenberg

Figs. 3-4 Gomphonema acuminatum Ehrenberg

Figs. 5-6, Gomphonema capitatum Ehrenberg 10-11

Fig. 7

Gomphonema cf. truncatum Ehrenberg

Figs. 8-9 Gomphonema truncatum Ehrenberg

Fig. 1 Lake Llebreta, sediment PYR58

Figs. 2, 5-6 Lake Posets, sediment PYR42

Figs. 3-4 Lake Burg

Fig. 7 Lake Burg, sediment BURG 1176

Figs. 8-9 Lake Angonella de Més Amunt, sediment PYR78

Figs. 10-11 Lake Gran de Mainera, epilithic EpiPYR70 

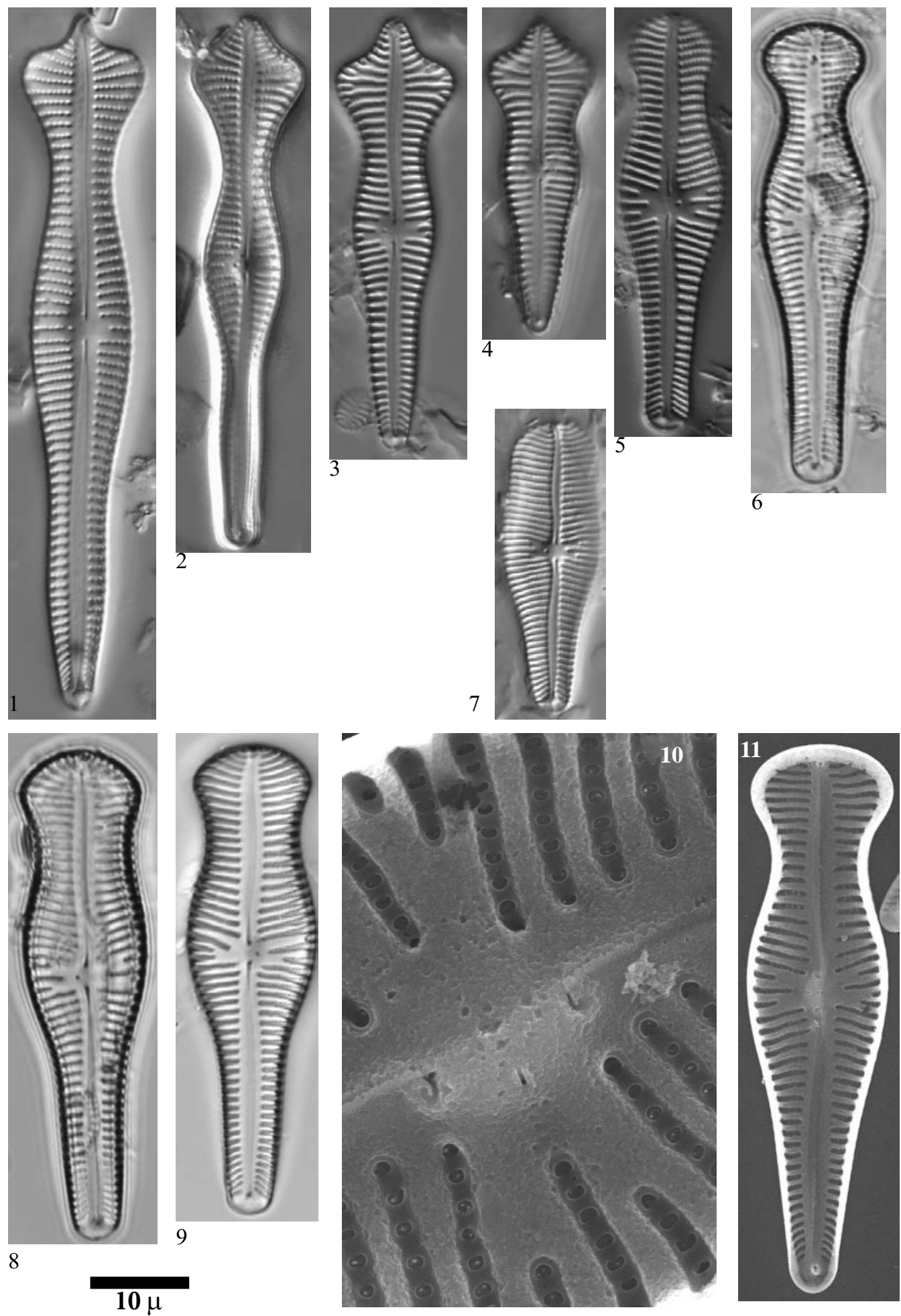
LM: x1500

Figs. 1-2

Figs. 3-5

Figs. 6

Figs. 7-8

Fig. 9-10

Fig. 11

Fig. 12

Figs. 13-25

Fig. 26-27
Gomphonema brebissonii Kützing

Gomphonema montanum Schumann

Gomphonema clavatum Ehrenberg

Gomphonema subclavatum (Grunow) Grunow

Gomphonema cf. subclavatum (Grunow) Grunow

Gomphonema sp. No. 1 Acherito

Gomphonema sp. No. 2 Laquettes

Gomphonema lateripunctatum Reichardt \& Lange-Bertalot

Gomphonema vibrio Ehrenberg

Lake PYR128

Fig. 1

Lake Gran del Pessó, sediment PYR56

Fig. 2

Figs. 3-4

Lake Cregüeña, sediment PYR49

Fig. 5

Fig. 6

Lake Més Amunt de Tristaina, epilithic EpiPYR86

Lake Compte, sediment PYR97

Fig. 7

Lake Forcat Inf., sediment PYR77

Fig. 8

Fig. 9

Fig. 10

Figs. 11, 24, 26-27

Figs. 12, 15-19, 21-22

Fig. 13

Fig. 14

Figs. 20, 25

Fig. 23
Lake Siscar, sediment PYR126

Lake Romedo de Dalt, sediment PYR85

Lake Inferior de la Gallina, sediment PYR87

Lake Acherito, sediment PYR01

Lake Les Laquettes, sediment PYR27

Lake Arratille, sediment PYR11

Lake Asnos, sediment PYR14

Lake Rond, sediment PYR72

Lake Gros de Camporrells, sediment PYR110 

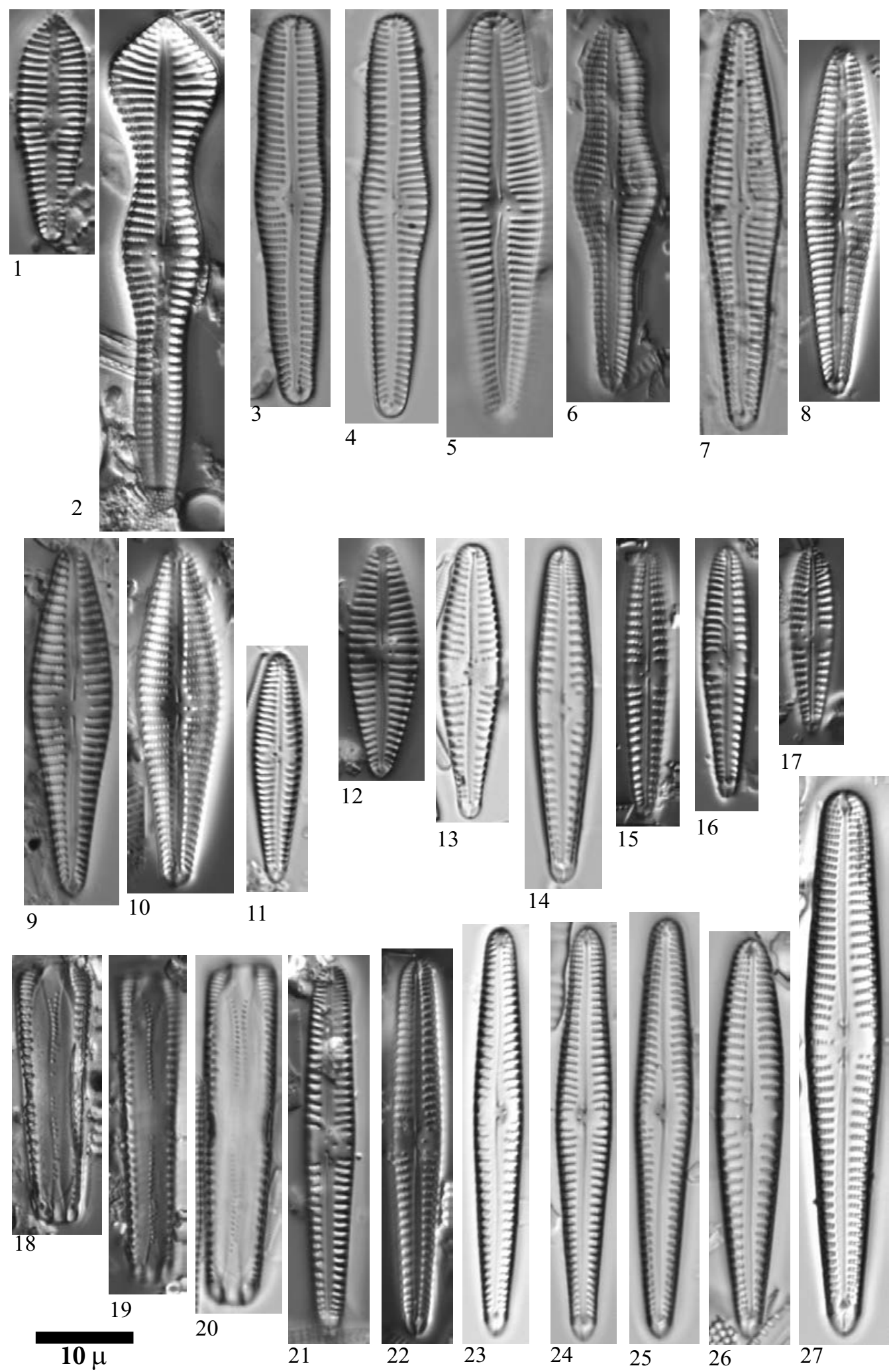

Limnetica, 36 (1): 127-395 (2017) 
Plate $112 \quad$ LM: $x 1500$

SEM: Figs. 4-5 x5000, Figs. 6-7 x15000, Fig. 8 x10000

Figs. 1-8 Gomphonema lateripunctatum Reichardt \& Lange-Bertalot

Figs. 1, 4, 6-7 Lake Roumassot, epilithic EpiPYR04

Fig. 2 Lake Les Laquettes, sediment PYR27

Figs. 5, 8 Lake Port Bielh, epilithic EpiPYR28 


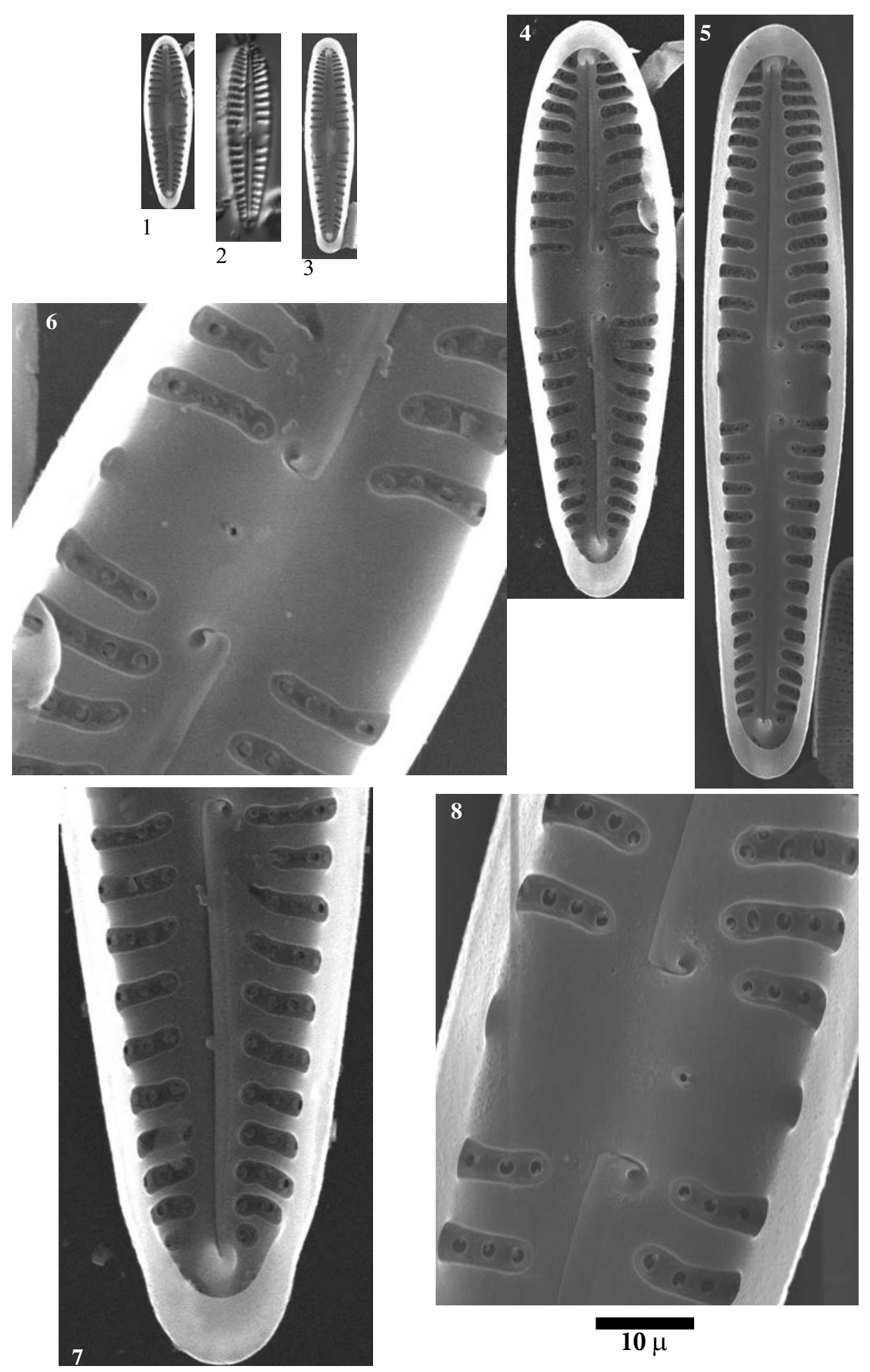

Limnetica, 36 (1): 127-395 (2017) 
Plate 113 LM: $x 1500$

SEM: Fig. 42 x 5000

Fig. 1-2 Gomphonema cf. designatum Reichardt

Fig. 3

Gomphonema cf. minusculum Krasske

Figs. 4-7

Gomphonema cf. pumilum (Grunow) Reichardt \& Lange-Bertalot

Figs. 8-10

Gomphonema cf. elegantissimum Reichardt \& Lange-Bertalot

Figs. 11-12 Gomphonema cf. pseudotenellum Lange-Bertalot

Figs. 13-17 Gomphonema cf. minusculum Krasske

Fig. $18 \quad$ Gomphonema sp. No. 4 Posets

Figs. 19-20 Gomphonema cf. lacus-vulcani Reichardt \& Lange-Bertalot

Fig. 21

Gomphonema sp. No. 5 Chelau

Figs. 22-30

Gomphonema sp. No. 6 Inferior

Fig. 31

Gomphonema sp. No. 7 Burg

Fig. 32

Gomphonema sp. No. 8 Laquettes

Fig. 33

Figs. 34-35

Gomphonema occultum Reichardt \& Lange-Bertalot

Fig. 36

Gomphonema tergestinum (Grunow) Fricke

Figs. 37-42 Gomphonema sp. No.9 Posets

Figs. 43-48 Gomphoneis cf. olivaceoides (Hustedt) Carter

Figs. 49-50 Gomphonema sp. No. 10 Inferior

Fig. 51

Gomphonema cf. rhombicum Fricke

Fig. 52

Gomphonema sp. No. 11 Ormiélas aff. G. tenue

Fig. 1 Lake Helado de Marboré, sediment PYR18

Fig. 2 Lake Pondiellos Sup., epilithic EpiPYR08

Fig. 3 Palaelake Burg, sediment BURG 804

Figs. 4, $45 \quad$ Lake Arratille, sediment PYR11

Fig. 5 Lake Tourrat, sediment PYR23

Figs. 6, 8-9, Lake Acherito, sediment PYR01 $12-13,30$

Fig. 7

Lake Compte, sediment PYR97

Fig. 10

Lake Gran de Mainera, sediment PYR70

Figs. 11, $32 \quad$ Lake Les Laquettes, sediment PYR27

Fig. 14 Lake Roumassot, sediment PYR04

Fig. 15 Lake Acherito, epilithic EpiPYR01

Sample information continued on the next page 

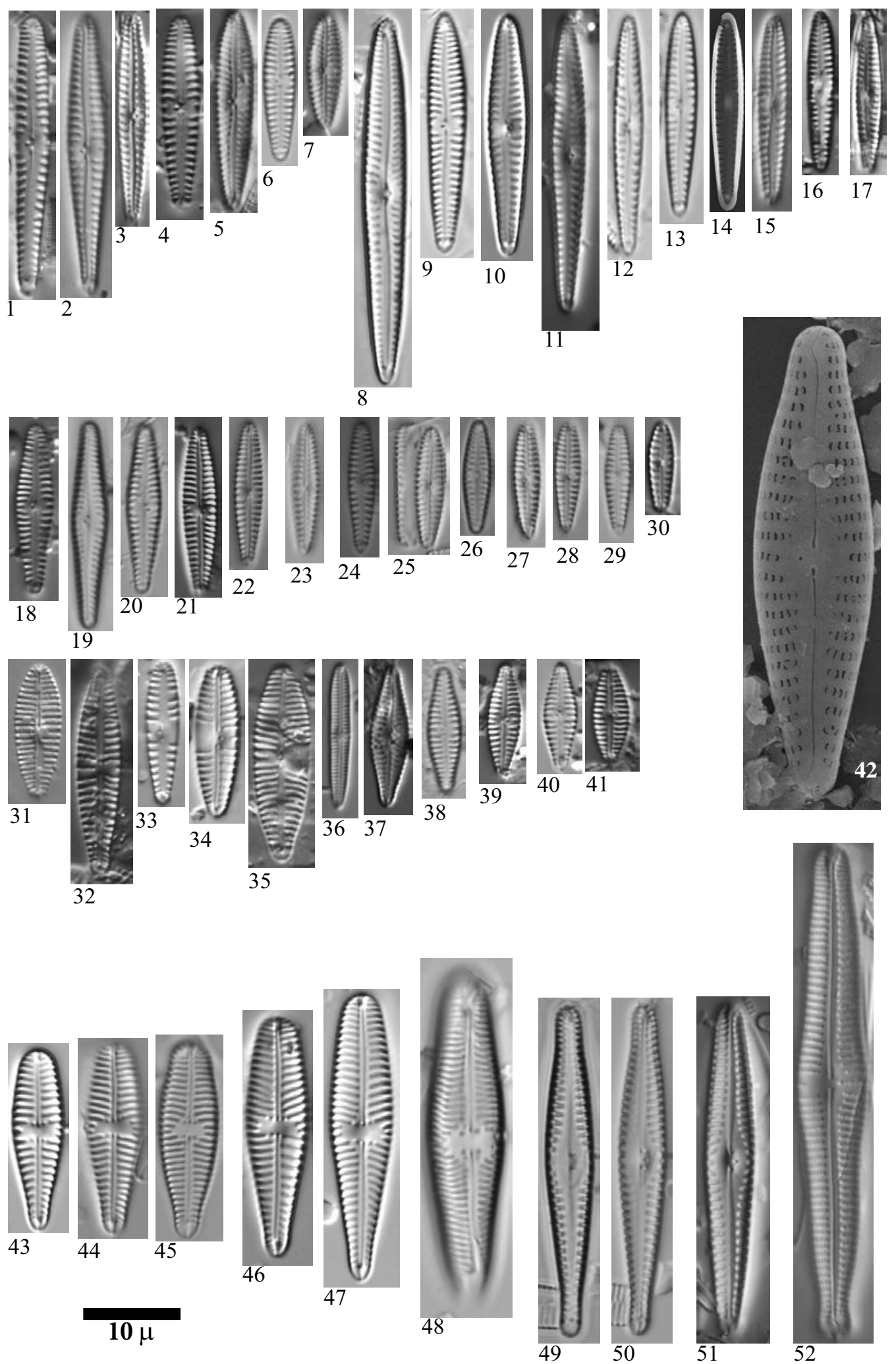

Limnetica, 36 (1): 127-395 (2017) 
Figs. 1-3

Figs. 4-7

Figs. 1-7
Gomphonema cf. minusculum Krasske

Gomphonema sp.

Lake Roumassot, sediment PYR04

Sample information of Plate 113

Figs. 16-17 Lake Port Bielh, sediment PYR28

Figs. 18-20, 37, 42 Lake Posets, sediment PYR42

Fig. 21

Lake Chelau Sup., sediment PYR41

Figs. 22-29, 49-50

Lake Inf. de la Gallina, sediment PYR87

Fig. 31

Lake Burg

Fig. 33

Lake Bleu epilithic EpiPYR22

Figs. 34, 43-44, Lake La Munia Sup., sediment PYR20 46-48

Fig. 35

Figs. 36-41

Fig. 51

Fig. 52
Lake Cap Long, sediment PYR24

Lake Compte epilithic EpiPYR97

Lake Trebens, sediment PYR114

Lake Pica Palomera epilithic EpiPYR52 

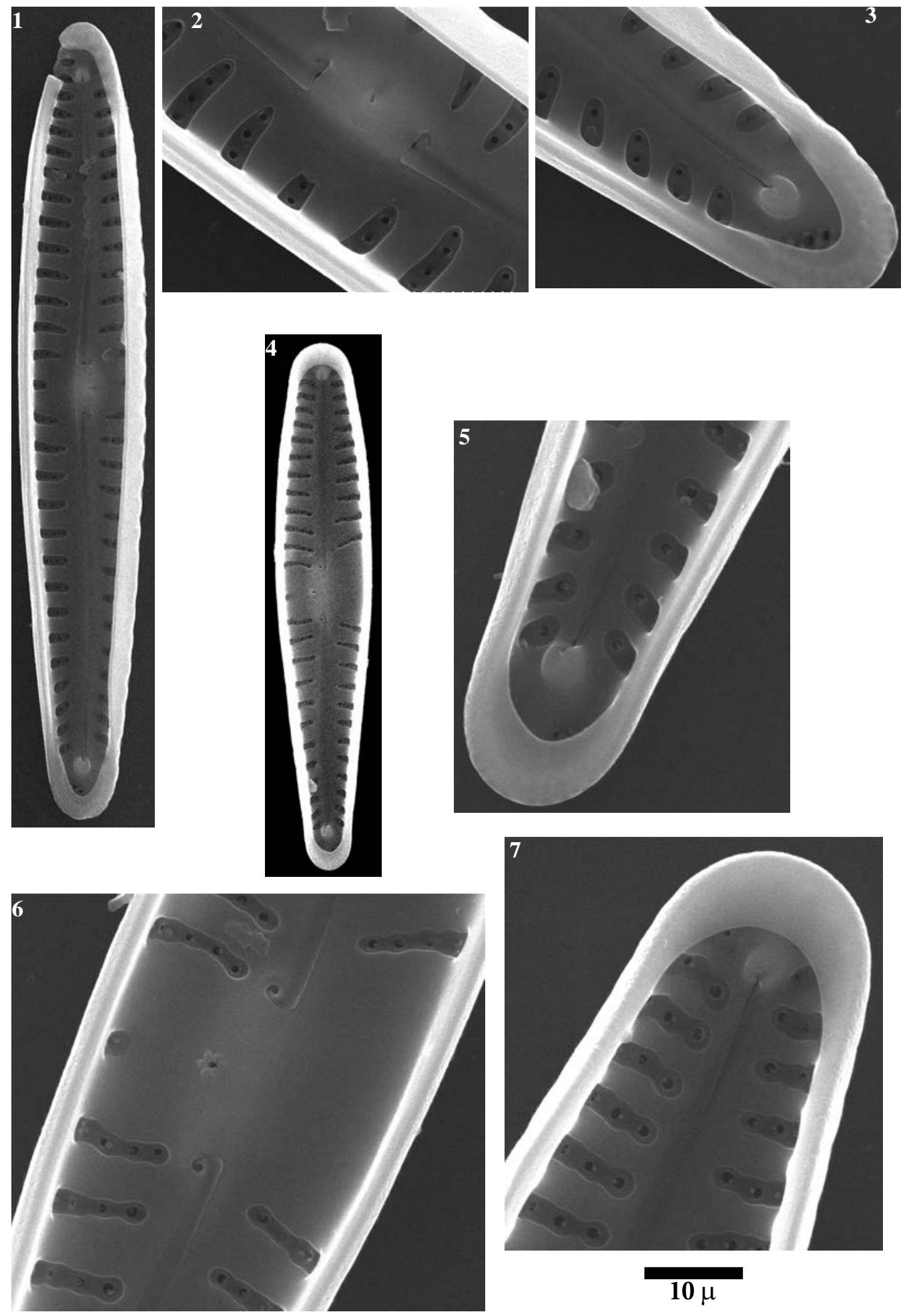
Fig. 1

Fig. 2

Fig. 3

Fig. 4

Fig. 5

Fig. 6

Figs. 7-10

Figs. 11-13

Figs. 14-15

Fig. 16

Fig. 17-21

Figs. 22-24

Fig. 25

Figs. 26-31

Figs. 32-33

Figs. 34-37

Figs. 38-44

Fig. 45-47

Fig. 48-50

Fig. 51

Fig. 1

Fig. 2

Figs. 3, 11-12, 15, 28

Fig. 4

Figs. 5, 22-23

Fig. 6

Fig. 7

Figs. 8-9, 18, 20-21

Figs. 10, 13, 19, 24

Figs. 14, 27

Figs. 16, 25

\section{Gomphonema sarcophagus Gregory}

Gomphonema sp. No. 12 Burg

Gomphonema lapponicum (Cleve) Cleve-Euler

Gomphonema micropus Kützing

Gomphonema sp. No. 13 Gerber

Gomphonema sp. No. 14 Cap Long

Gomphonema sp. No. 15 Coronas

Gomphonema cf. cymbelliclinum Reichardt \& Lange-Berta-

lot

Gomphonema parvulum (Kützing) Kützing sensu lato

Gomphonema parvulum (Kützing) Kützing sensu lato

Gomphonema sp. No. 16 Estagnol

Gomphonema sp. No. 17 Gerber

Gomphonema cf. gracile Ehrenberg

Gomphonema cf. hebridense Gregory

Gomphonema auritum Braun

Gomphonema sp. No. 20 Laquettes

Gomphonema spp

aff. Gomphonema parvulum (Kützing) Kützing sensu lato

Gomphonema cf. acidoclinatum Lange-Bertalot \& Reichardt

Gomphonema sp. No. 18 Laquettes

Gomphonema sp. No. 19 Laquettes

Lake Burg, sediment 970

Lake Burg, sediment 473

Lake Cregüeña, sediment PYR49

Lake Rond, sediment PYR72

Lake Gerber, sediment PYR63

Lake Cap Long, epilithic EpiPYR24

Lake Coronas, sediment PYR47

Lake L'Estagnol, sediment PYR119

Lake Inf. de la Gallina, sediment PYR87

Lake Bachimala Sup., sediment PYR31

Lake Mariola, sediment PYR80

Sample information continued on the next page 

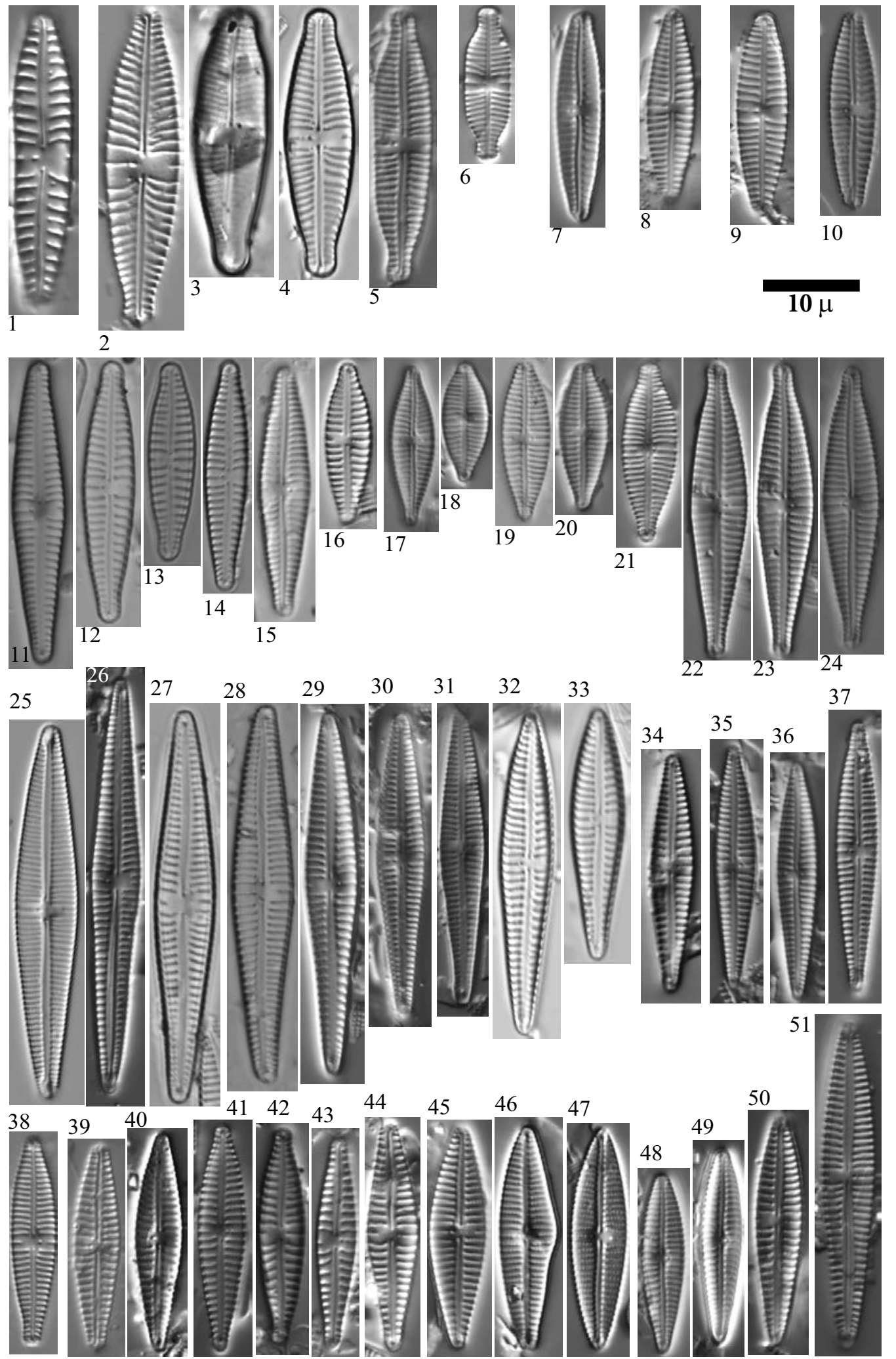

Limnetica, 36 (1): 127-395 (2017) 
Figs. 1-4

Gomphonema hebridense Gregory

Figs. 1-4

Lake Port Bielh, sediment EpiPYR28

Sample information of Plate 115

Figs. 17, 38-39 Lake Burg

Figs. 26, 29-31, 35-37, Lake Les Laquettes, sediment PYR27 $41-43,48-51$

Fig. 32

Lake Arratille, sediment PYR11

Fig. 33

Lake Acherito, sediment PYR01

Figs. 34,40

Lake Sen, sediment PYR40

Fig. 44

Lake Eriste, sediment PYR43

Fig. 45

Lake Pixón, sediment PYR44

Figs. 46-47

Lake Pica, sediment PYR100 

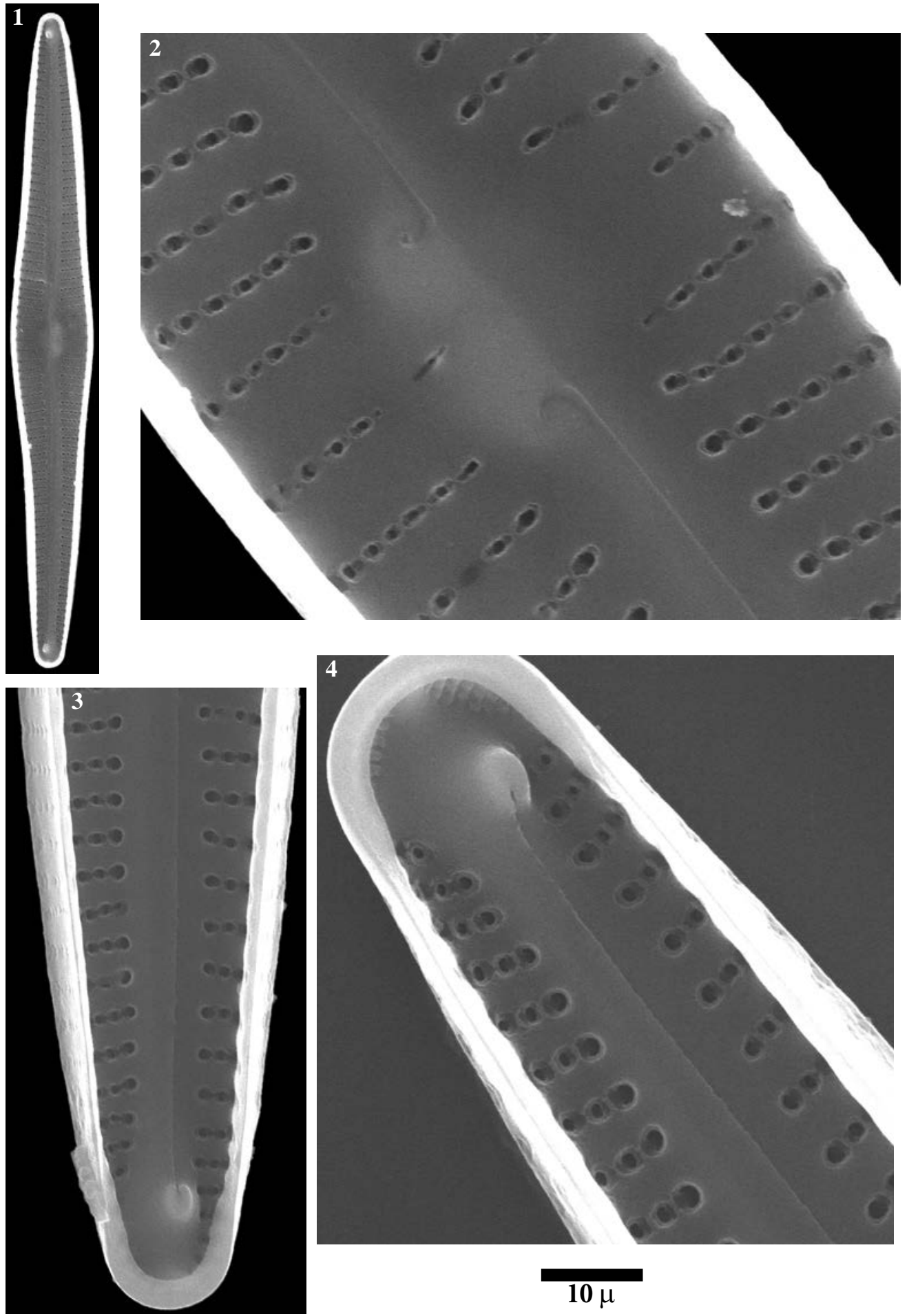
SEM: 4-7, 8, 13, 14 x8000 $20 \times 5000$

Figs. 1-6

Figs. 7-15

Figs. 16-20

Figs. 1, 3, 19-20

Fig. 2

Fig. 4

Fig. 5

Fig. 6

Fig. 7

Fig. 8

Figs. 9, 15

Fig. 10

Fig. 11

Figs. 12, 16-18

Fig. 13

Fig. 14
Nitzschia sp. No. 4 Airoto

Nitzschia spp

Fig. 9-10, 12 Nitzschia sp. No. 6 Sen

Fig. 11 Nitzschia sp. No. 5 Arratille
Nitzschia sp. No. 1 Sen

Lake Posets, sediment PYR42

Lake Illa, sediment PYR66

Lake Redon, sediment REDOM

Lake Roumassot, epilithic EpiPYR04

Lake Roumassot, sediment PYR04

Lake Port Bielh, epilithic EpiPYR28

Lake Garbet, sediment PYR81

Lake Gran de Mainera, sediment PYR70

Lake Rond, sediment PYR72

Lake Arratille, sediment PYR11

Lake Sen, sediment PYR40

Lake Laurenti, sediment PYR111

Lake Arnales, epilithic EpiPYR09 

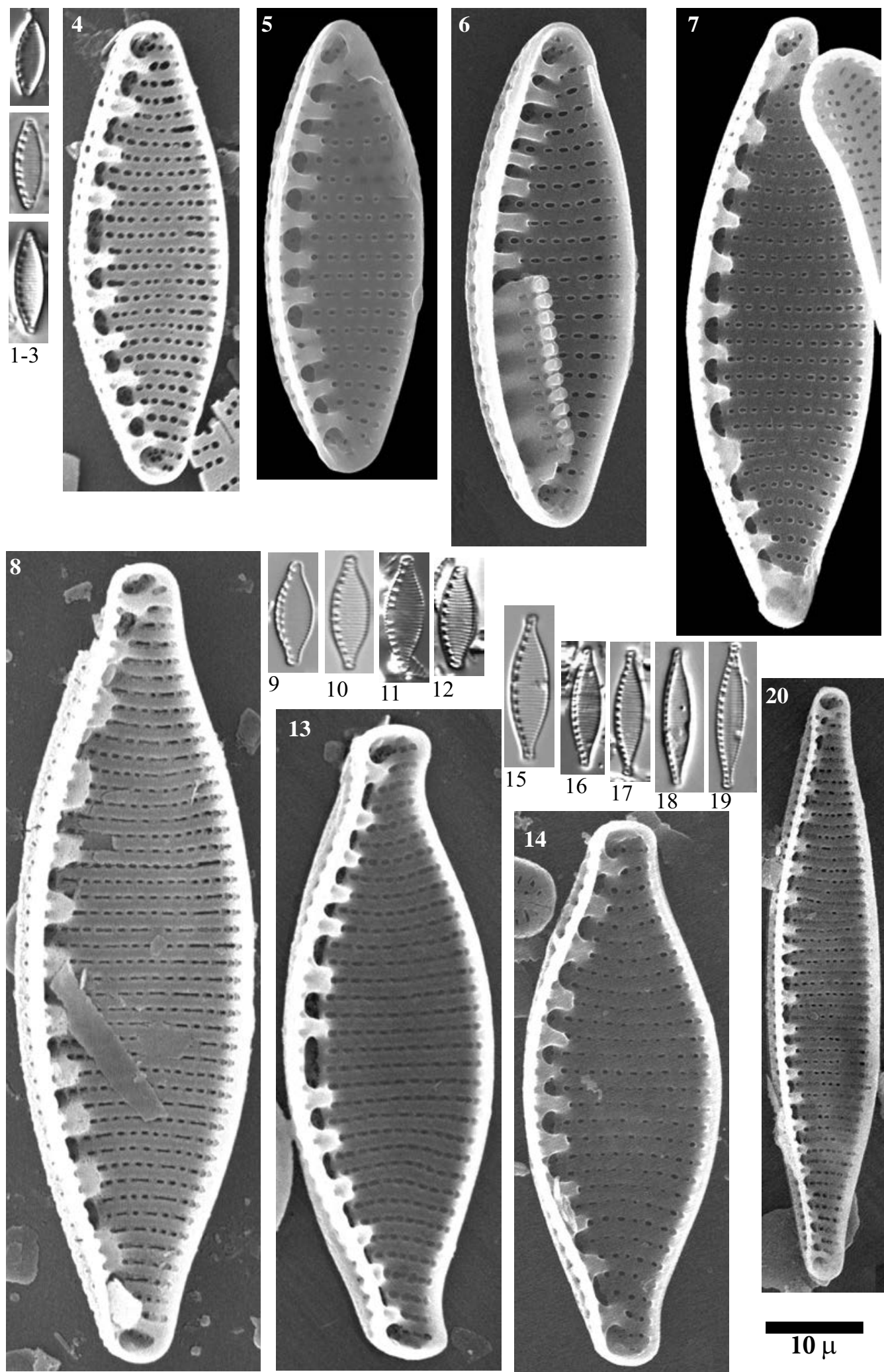

Limnetica, 36 (1): 127-395 (2017) 
Figs. 1-4

Figs. 5-6

Figs. 7-13

Figs. 14, 27-30

Figs. 15-18

Fig. 19-22

Figs. 23-26, 31

Fig. 1

Fig. 2

Fig. 3

Figs. 4-6, 16

Fig. 7

Fig. 8

Figs. 9-13, 14,

28-30

Figs. 15,17

Figs. 18, 20-22

Fig. 19

Fig. 23

Fig. 24

Figs. 25-26

Fig. 27

Fig. 31
Nitzschia cf. frustulum (Kützing) Grunow

Nitzschia cf. inconspicua Grunow

Nitzschia cf. alpina Hustedt

Nitzschia sp

Nitzschia acidoclinata Lange-Bertalot

Nitzschia cf. perminuta (Grunow) Peragallo M1

Nitzschia cf. perminuta (Grunow) Peragallo M2

Lake Roumassot, epilithic EpiPYR04

Lake Etriste, sediment PYR43

Lake Estom, sediment PYR15

Lake Burg

Lake Filià, sediment PYR71

Lake Llebreta, sediment PYR58

Lake Posets, sediment PYR42

Lake Bersau, sediment PYR03

Lake Inf. de la Gallina, sediment PYR87

Lake Arratille, sediment PYR11

Lake Sen, sediment PYR40

Lake Gelat Bergús, sediment PYR65

Lake Basa de la Mora, sediment PYR32

Lake Redon, sediment REDOM

Lake Port Bielh, epilithic EpiPYR28 

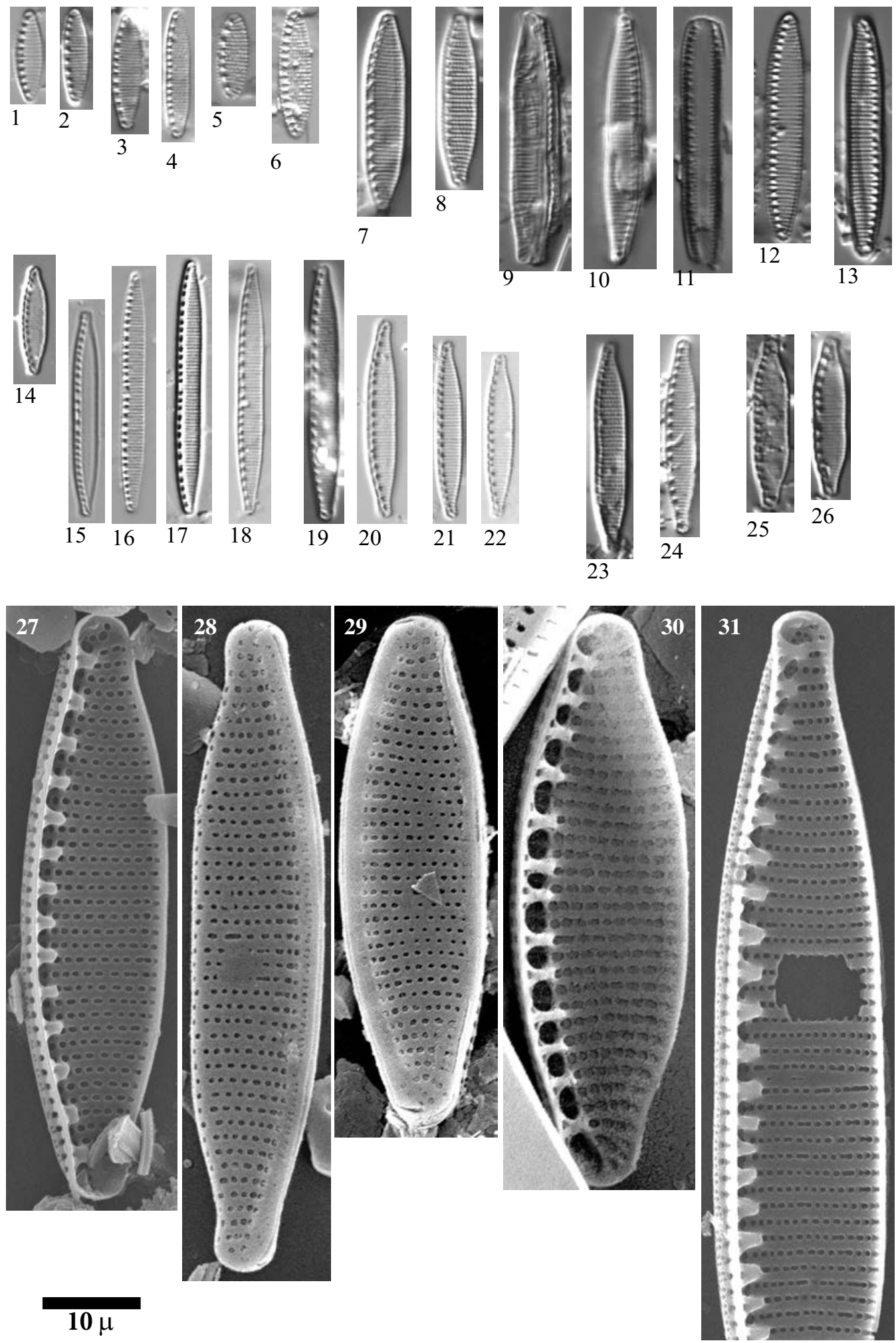
Plate $119 \quad$ LM: x1500

SEM: Figs. $8-9$ x6000, Fig. 10 x4500

Fig. $1 \quad$ Nitzschia sp. No. 16 Mora

Figs. 2-3 Nitzschia cf. pumila Hustedt

Figs. 4-5, $10 \quad$ Nitzschia cf. pumila Hustedt

Figs. 6-9 Nitzschia sp. No. 2 Posets

Figs. 11-17 Nitzschia cf. bryophila (Hustedt) Hustedt

Figs. 18-23 Nitzschia sp. No. 15 Burg, aff. bryophila (Hustedt) Hustedt

Figs. 24-28 Nitzschia cf. bryophila (Hustedt) Hustedt

Fig. 29 Nitzschia sp.

Fig. $30 \quad$ Nitzschia sp. No. 13 Coronas

Fig. $31 \quad$ Nitzschia sp. No. 3 Airoto

Figs. 32-33 Nitzschia palea var. debilis (Kützing) Grunow

Fig. 1 Lake Basa de la Mora, sediment PYR32

Fig. 2 Lake Arnales, sediment PYR09

Fig. 3 Lake Burg, sediment BURG 1116

Figs. 4-8, 29 Lake Posets, sediment PYR42

Fig. 9 Lake Pondiellos Sup., epilithic EpiPYR08

Fig. $10 \quad$ Lake Port Bielh, epilithic EpiPYR28

Fig. 11 Lake Inf. de la Gallina, sediment PYR87

Fig. 12 Lake Illa, epilithic EpiPYR66

Figs. 18-23 Lake Burg

Figs. 24-26 Lake Sen, sediment PYR40

Fig. 27 Lake Més Amunt de Tristaina, sediment PYR86

Fig. 28 Lake Pixón, sediment PYR44

Fig. $30 \quad$ Lake Coronas, epilithic EpiPYR47

Fig. $31 \quad$ Lake Airoto, sediment PYR73

Fig. 32 Lake Pondiellos Sup., sediment PYR08

Fig. 33 Lake Lliterola, sediment PYR33 

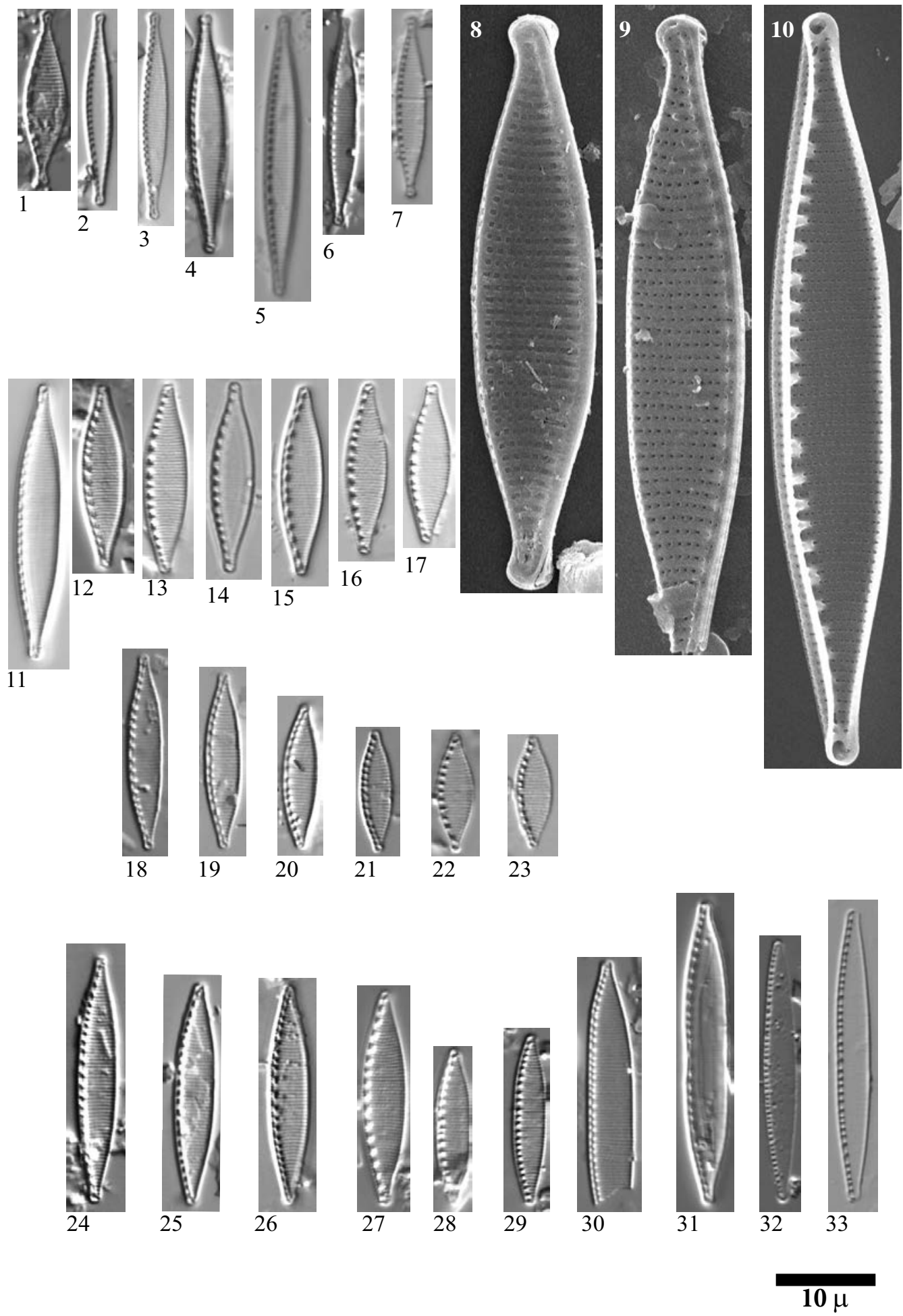
Plate $120 \quad$ LM: $\times 1500$

SEM x3000

Figs. 1-3 Nitzschia sp. No. 7 Posets

Figs. 4-6 Nitzschia cf. paleacea (palacea) Grunow

Figs. 7-8 Nitzschia sp. No. 11 Burg

Figs. 9-10 Nitzschia sp. No. 12 Burg

Figs. 11-12 Nitzschia sp. No. 8 Bergeus

Figs. 13-16 Nitzschia gracilis Hantzsch

Fig. $17 \quad$ Nitzschia cf. linearis var. subtilis Hustedt

Figs. 18-19 Nitzschia pura Hustedt

Figs. 20-22 Nitzschia cf. dissipata (Kützing) Grunow

Fig. 23 Nitzschia garrensis Hustedt

Fig. $24 \quad$ Nitzschia sp. No.10 Mora

Fig. $25 \quad$ Nitzschia rectiformis Hustedt

Fig. $26 \quad$ Nitzschia sp. No.9 Mora

Fig. $27 \quad$ Nitzschia sp. No. 14 Burg

Fig. 28 cf. Nitzschia amphibia var. fossilis Grunow

Figs. 29-30 Hantzschia cf. amphioxys (Ehrenberg) Grunow

Figs. 31-32 Hantzschia cf. rhaetica Meister

Figs. 1-3, 18, Lake Posets, sediment PYR42 Fig. $20 \quad$ Lake Sen, sediment PYR40

21, 28-29

Figs. 4-6

Lake Compte, sediment

Fig. 22

Lake Albe, sediment PYR96

Fig. 7 L. Burg, sediment BURG 1006

Fig. 23

Lake Acherito, sediment PYR01

Fig. 8 L. Burg, sediment BURG 1007

Figs. 9-10 Lake Burg

Figs. 24, 26

L. Basa de la Mora, sediment PYR32

Fig. 25

L. Més Amunt de Tristaina, sediment PYR86

Fig. 11

Lake Eriste, sediment PYR43

Fig. 27

L. Burg, sediment BURG 755

Figs. 12, 30

L. Gelat Bergús, sed. PYR65

Figs. 31-32

L. Burg, sediment BURG 1195

Fig. 13

L. Inf. Gallina, epilit. EpiPYR87

Fig. 14 L. Urdiceto, sediment PYR125

Fig. 15 Lake Illa, sediment PYR66

Fig. 16 L. L’Estagnol, epil. EpiPYR119

Fig. 17 L. Pondiellos Sup., epil. EpiPYR08

Fig. 19 Lake Mariola, sediment PYR80 

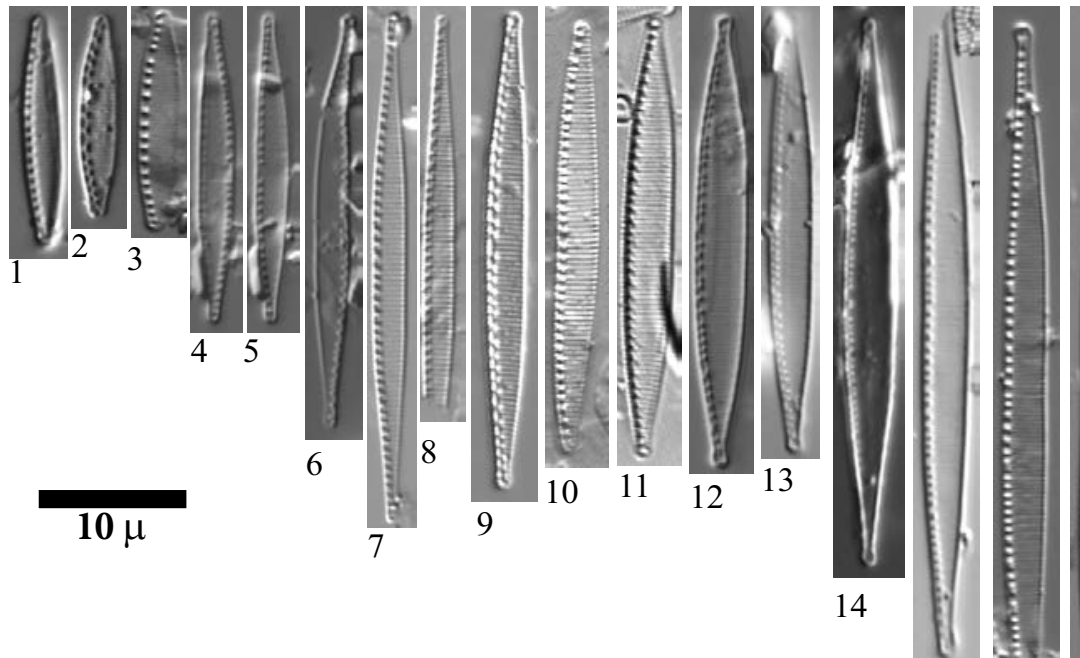

23
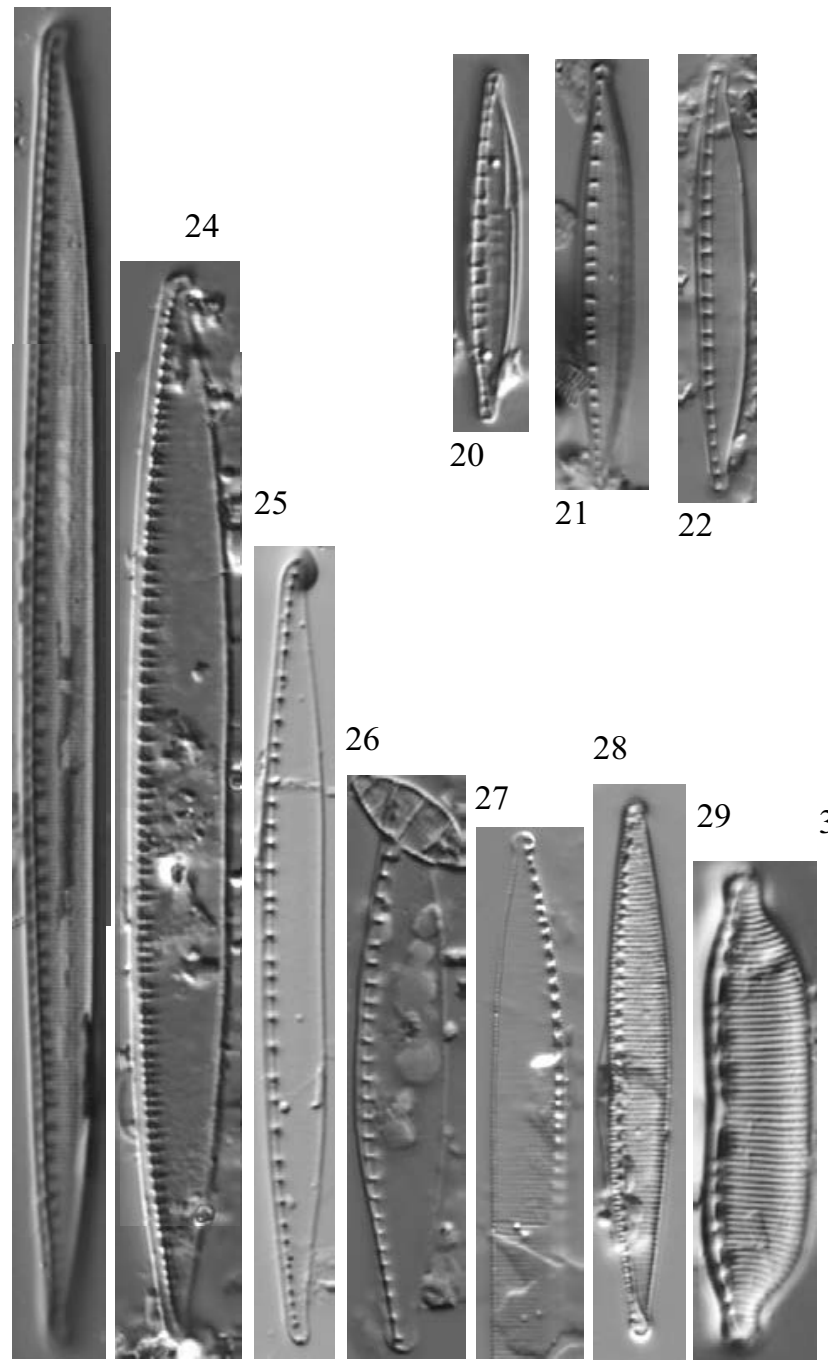

30
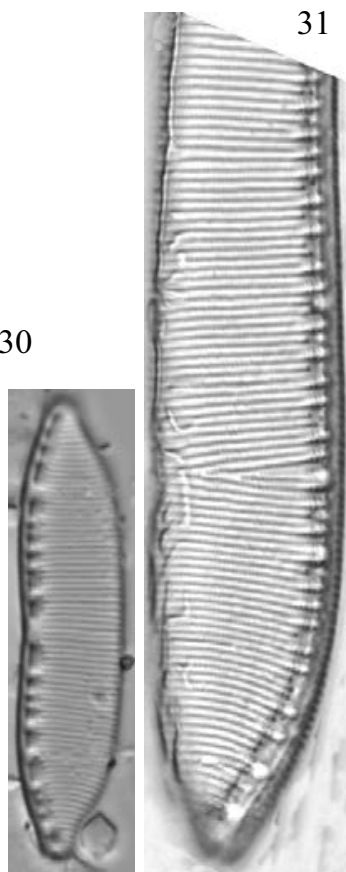

32

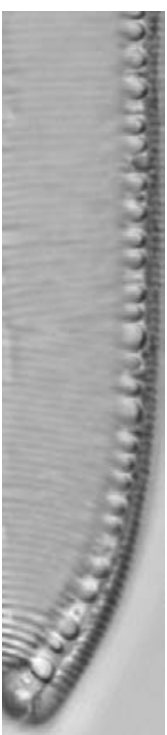

Limnetica, 36 (1): 127-395 (2017) 
Plate $121 \quad$ LM: $x 1500$

SEM: Figs. 6, 7 x3000, Fig. 8 x400, Fig. 9 x2000, Fig. $10 \times 10000$

Figs. 1-7 Nitzschia angustata (W. Smith) Grunow

Figs. 8-10 Nitzschia rectiformis Hustedt

Figs. 1, 3-5 Lake Estom, sediment PYR15

Fig. 2 Lake Posets, sediment PYR42

Figs. 6-7 Lake Port Bielh, epilithic EpiPYR28

Figs. 8-10 Lake Redon, sediment REDOM 

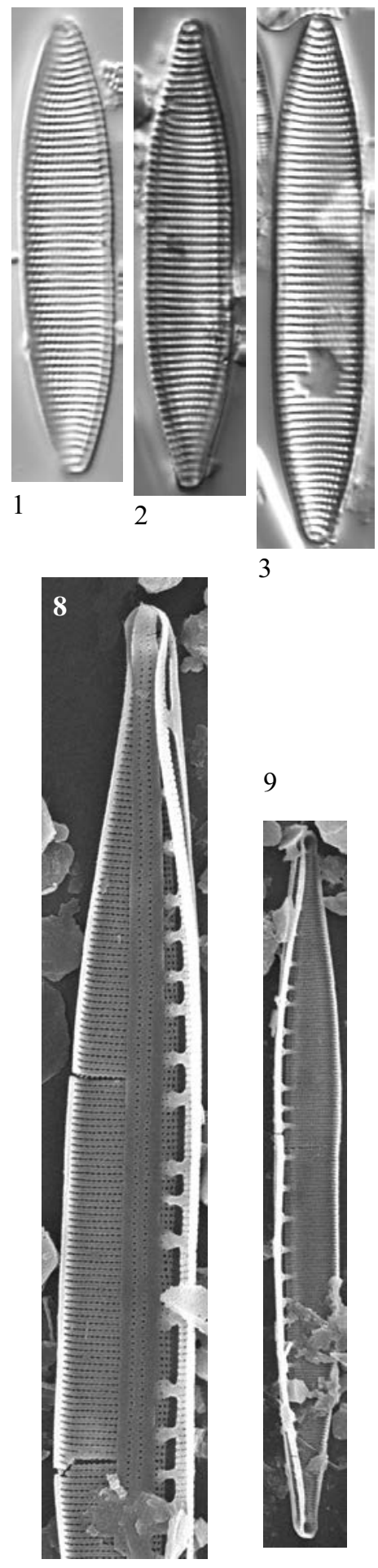
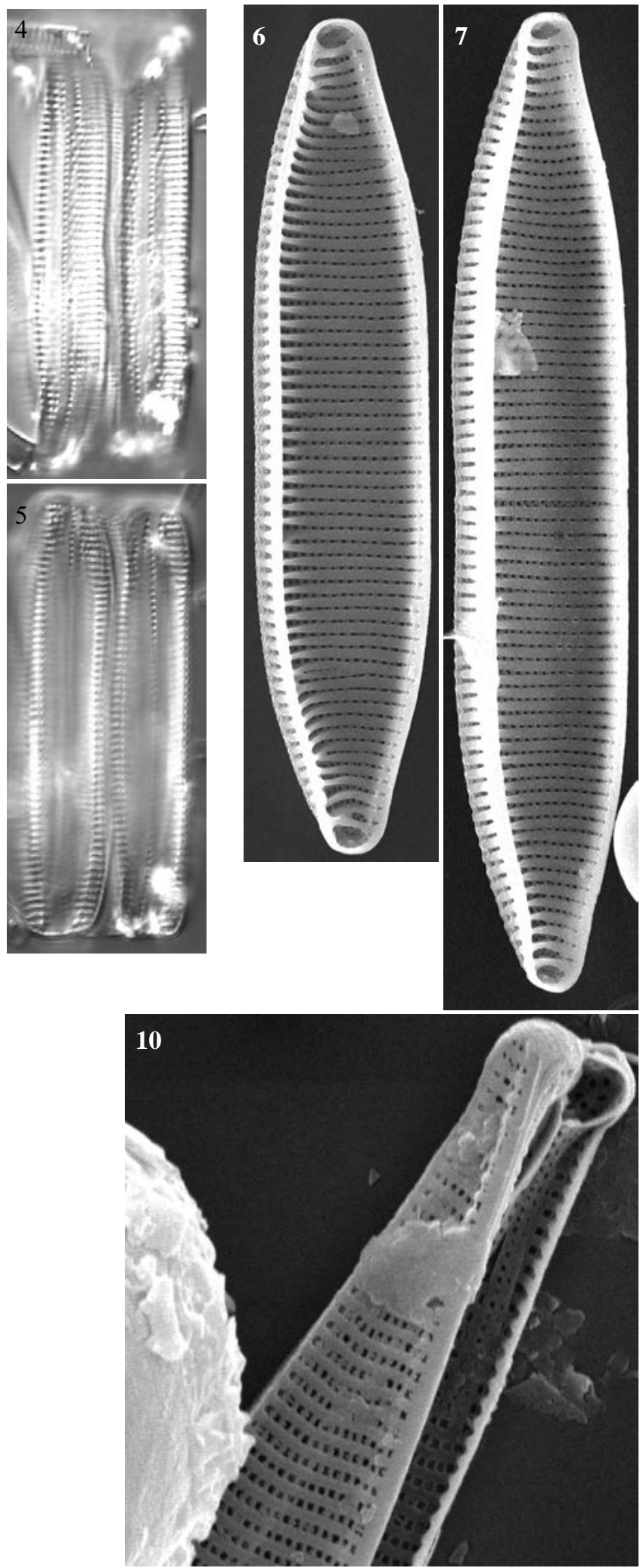

Limnetica, 36 (1): 127-395 (2017) 
SEM: Figs. 6,7,12 x10000, Figs. 13-15 x5000

Figs. 1-15

Denticula tenuis Kützing

Figs. 1-2, 5, 8-9, 12, 14 Lake Posets, sediment PYR42

Figs. 3-4, 10-11

Lake Sen, sediment PYR40

Figs. 6, 7

Lake Roumassot, sediment PYR04

Figs. 12, 15

Lake Pondiellos Sup., epilithic EpiPYR08

Fig. 13

Lake Port Bielh, epilithic EpiPYR28 

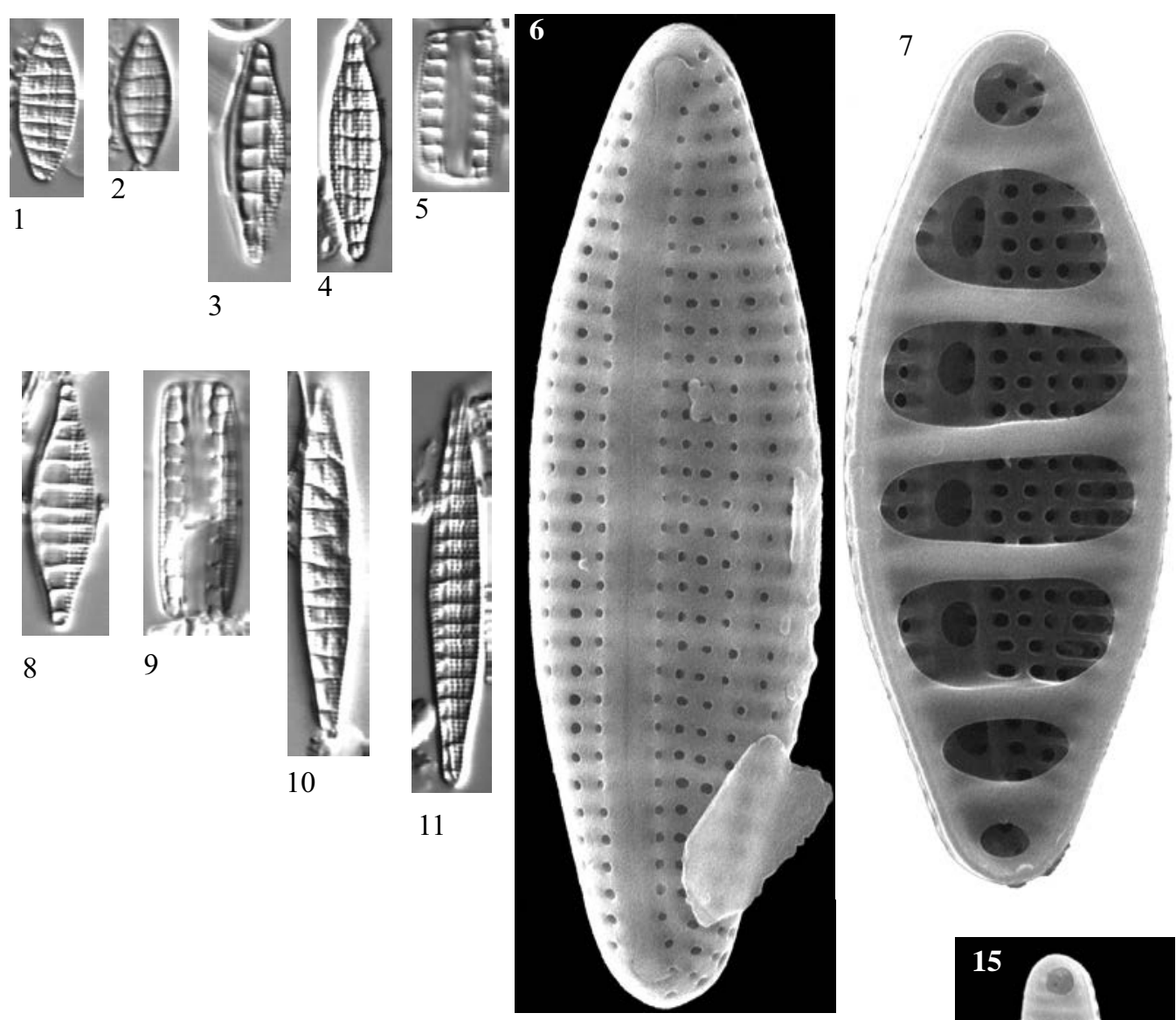

11
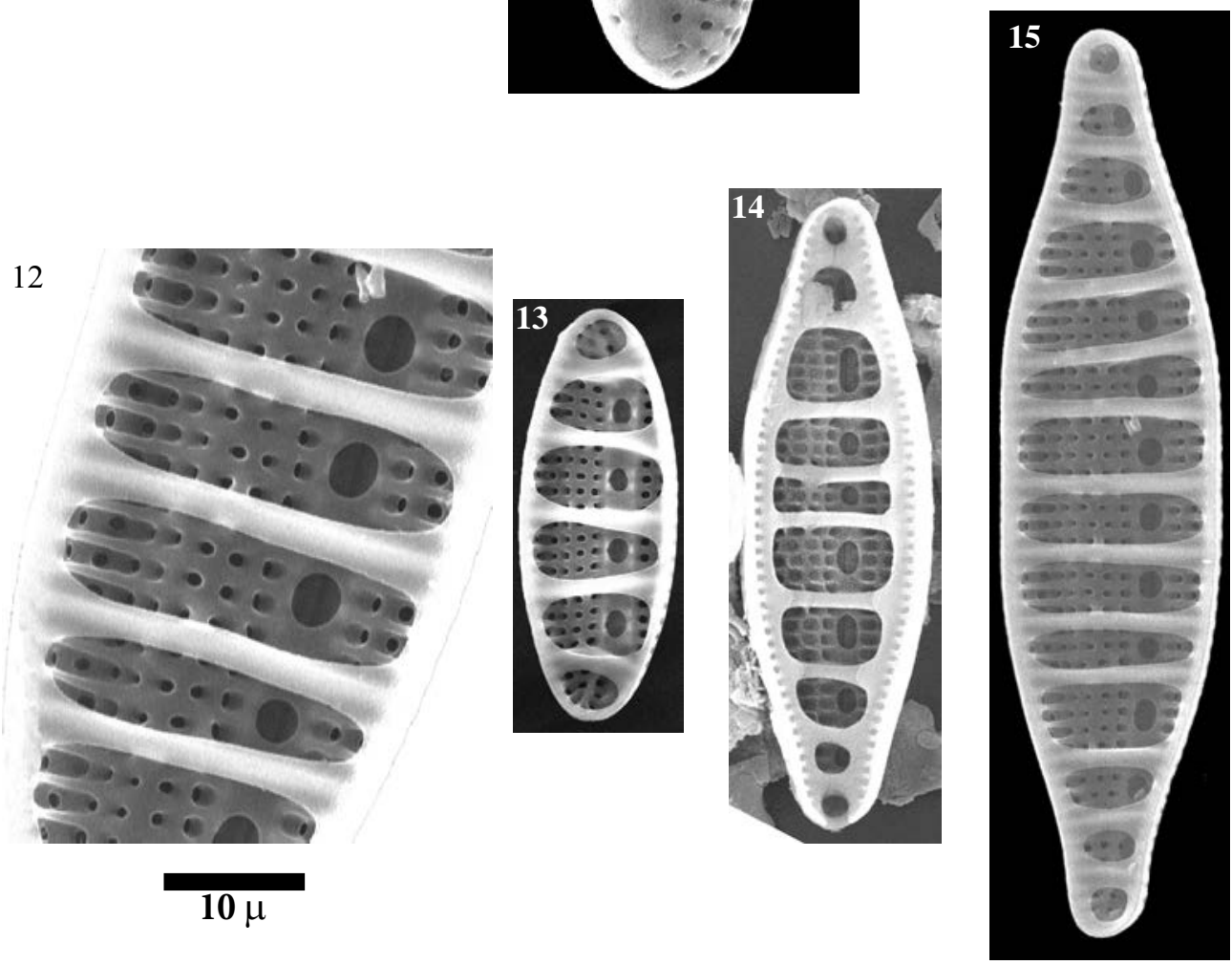

Limnetica, 36 (1): 127-395 (2017) 
Plate $123 \quad$ LM: $x 1500$

SEM: x3000

Figs. 1-6 Epithemia turgida (Ehrenberg) Kützing

Figs. 7-10 Epithemia cf. adnata (Kützing) Brébisson

Figs. 11-17 Epithemia sorex Kützing

Figs. 1-9, $11 \quad$ Lake Burg, sediment BURG 1115

Figs. 10, 15-16 Lake Burg, sediment BURG 1104

Fig. 12 Lake Burg, sediment BURG 1116

Fig. 17 Lake Roumassot, sediment PYR04 

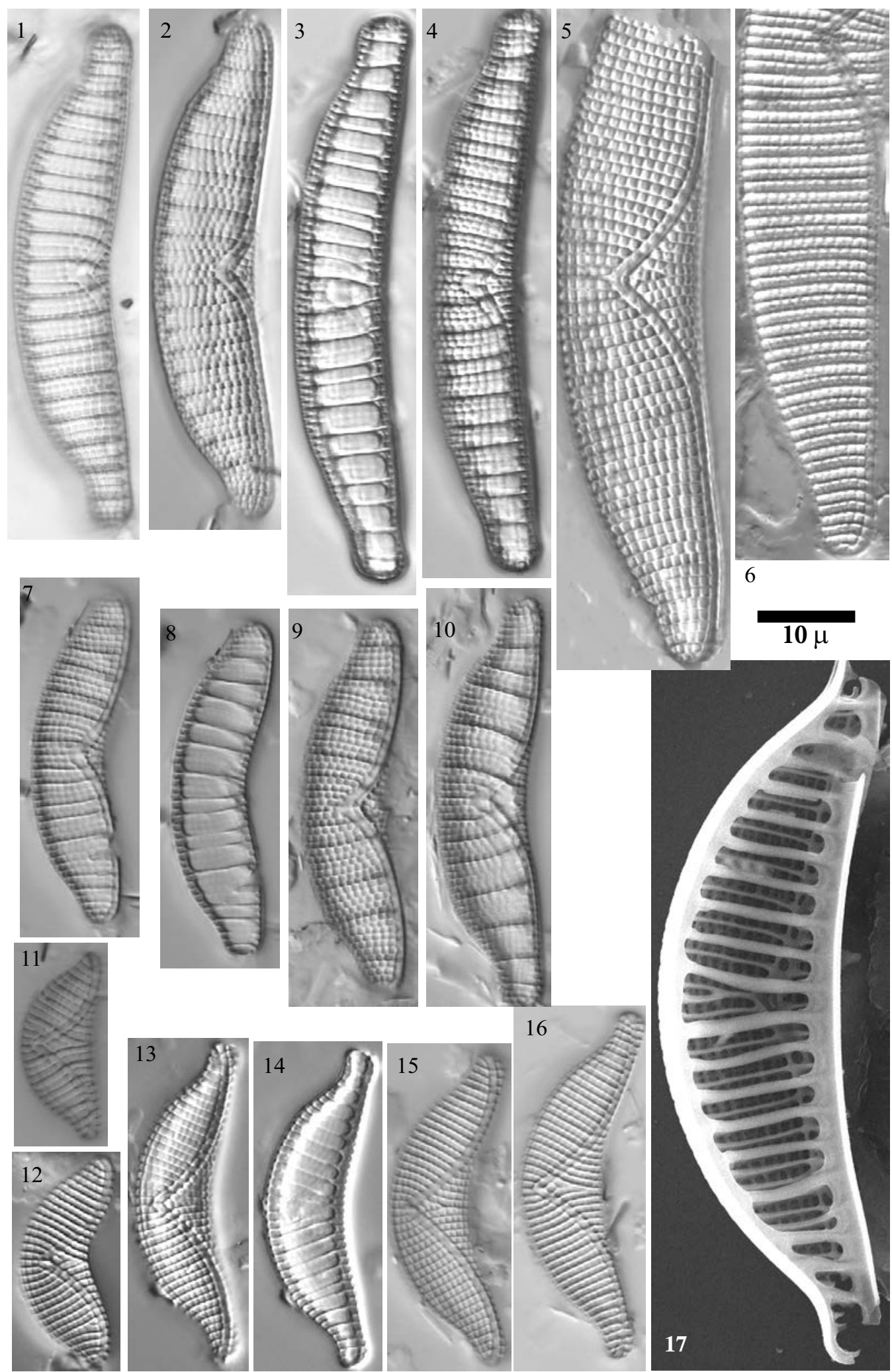

Limnetica, 36 (1): 127-395 (2017) 
Plate $124 \quad$ LM: $x 1500$

Figs. 1-2, Epithemia goeppertiana Hilse

$7-8$

Fig. 3 Epithemia cf. goeppertiana Hilse

Figs. 4-6 Rhopalodia gibba (Ehrenberg) Müller

Fig. 9 Epithemia cistula (Ehrenberg) Ralfs

Figs, 1-2, 7-8 Lake Estom, sediment PYR15

Fig. 3 Lake Burg, sediment sample

Figs. 4-6 Lake Burg, sediment BURG 519

Fig. 9 Lake Acherito, sediment PYR01 

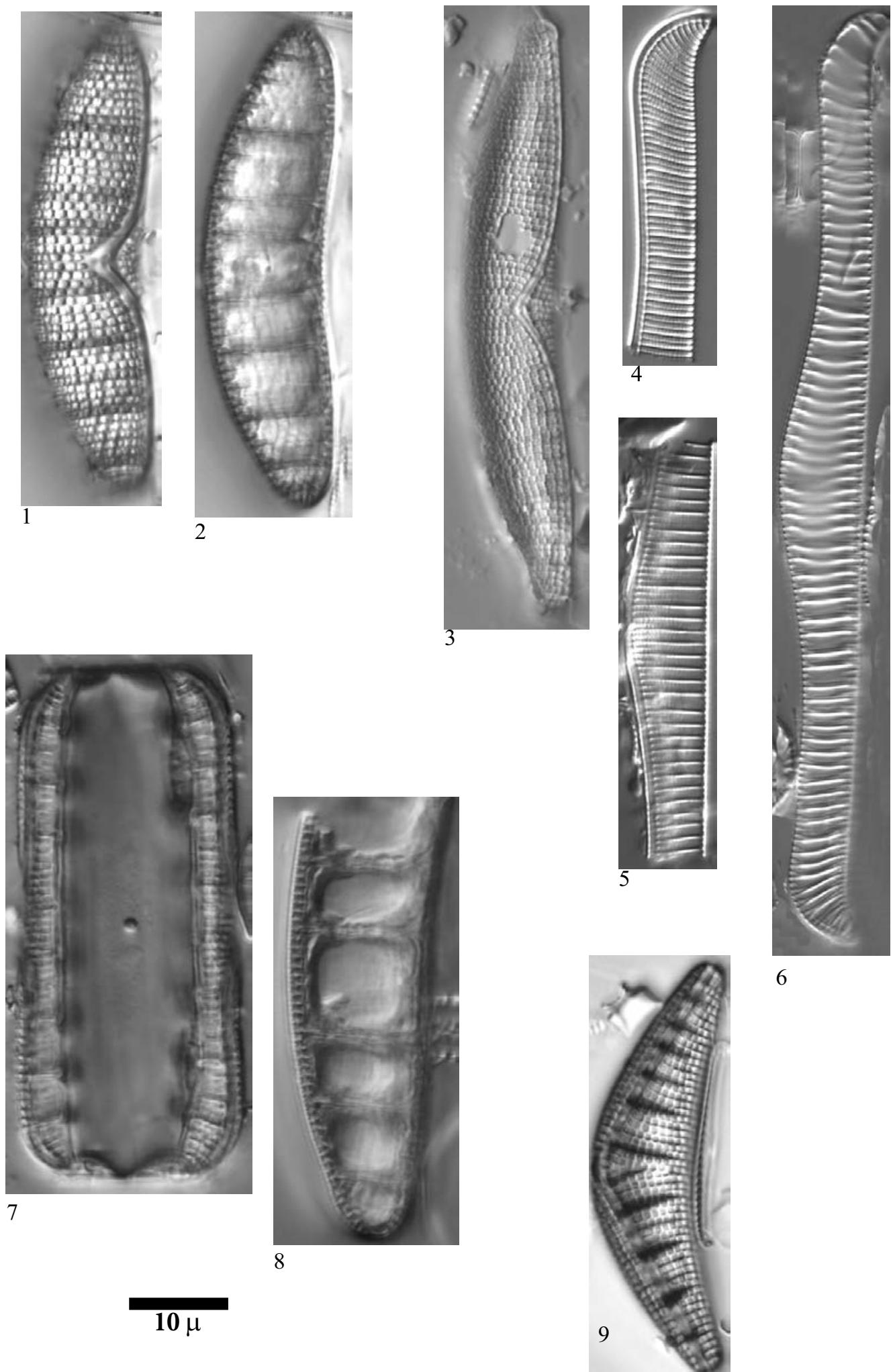

6

Limnetica, 36 (1): 127-395 (2017) 
Plate 125 LM: $x 1500$

SEM: Fig. 2 x8000, Fig. 3 x4000

Figs. 1-3 Surirella cf. roba Leclercq

Figs. 4-5 Surirella cf. bohemica Maly

Figs. 6-7 Surirella angusta Kützing

Fig. $8 \quad$ Surirella helvetica Brun

Figs. 1-2 Lake Posets, sediment PYR42

Fig. 3 Lake Redon, sediment REDOM

Figs. 4-5 Lake Forcat Inf., sediment PYR77

Fig. 6 Lake Coronas, sediment PYR47

Fig. 7 Lake Les Laquettes, sediment PYR27

Fig. 8 Lake Tourrat, sediment PYR23 

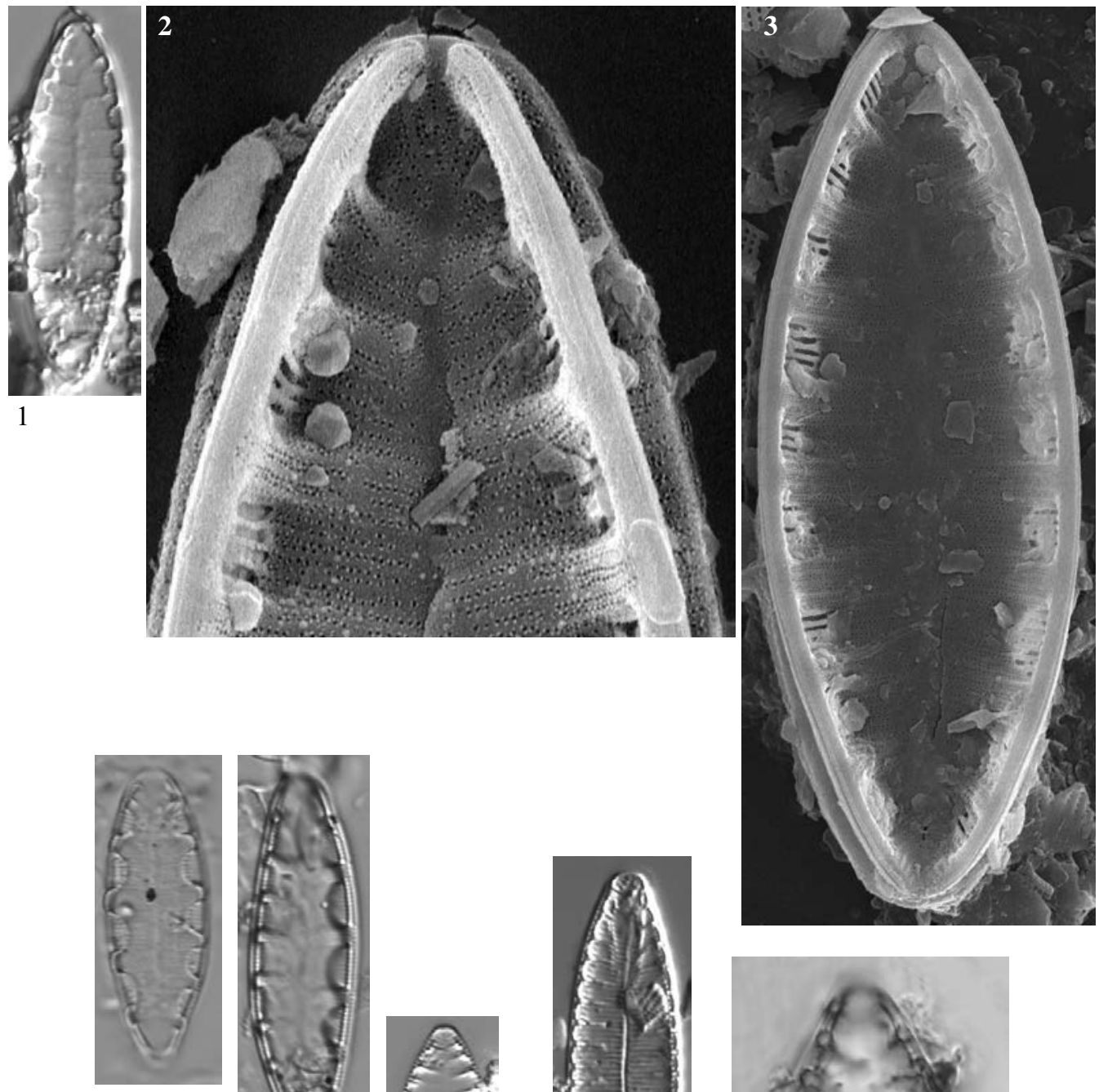

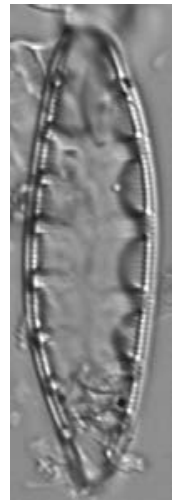

5

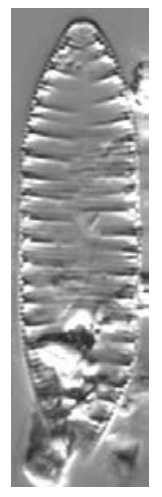

6

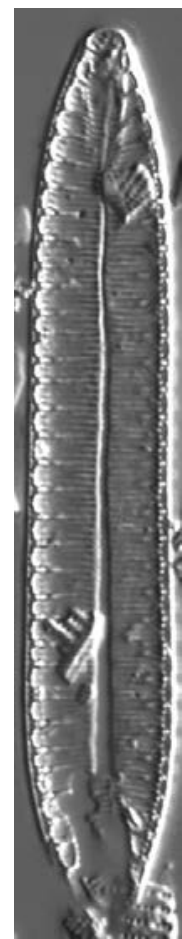

7

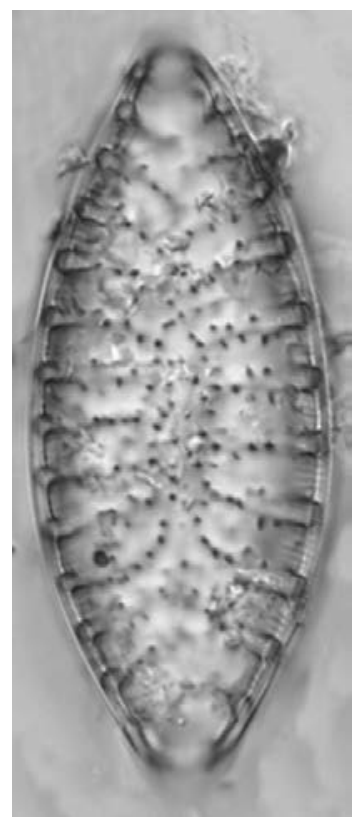

8

Limnetica, 36 (1): 127-395 (2017) 
Plate $126 \quad$ LM: x1500

Fig. 1

Fig. 2

Figs. 3-5

Figs. 6-11

Figs. 1, 7

Fig. 2

Figs. 3-6

Figs. 8-11
Surirella aff. robusta Ehrenberg

Surirella cf. linearis Smith

Stenopterobia densestriata (Hustedt) Krammer

Stenopterobia delicatissima (Lewis) Van Heurck
Lake Les Laquettes, sediment PYR27

Lake Gran de Mainera, sediment PYR70

Lake Romedo de Dalt, sediment PYR85

Lake Bleu de Rabassoles, sediment PYR112 

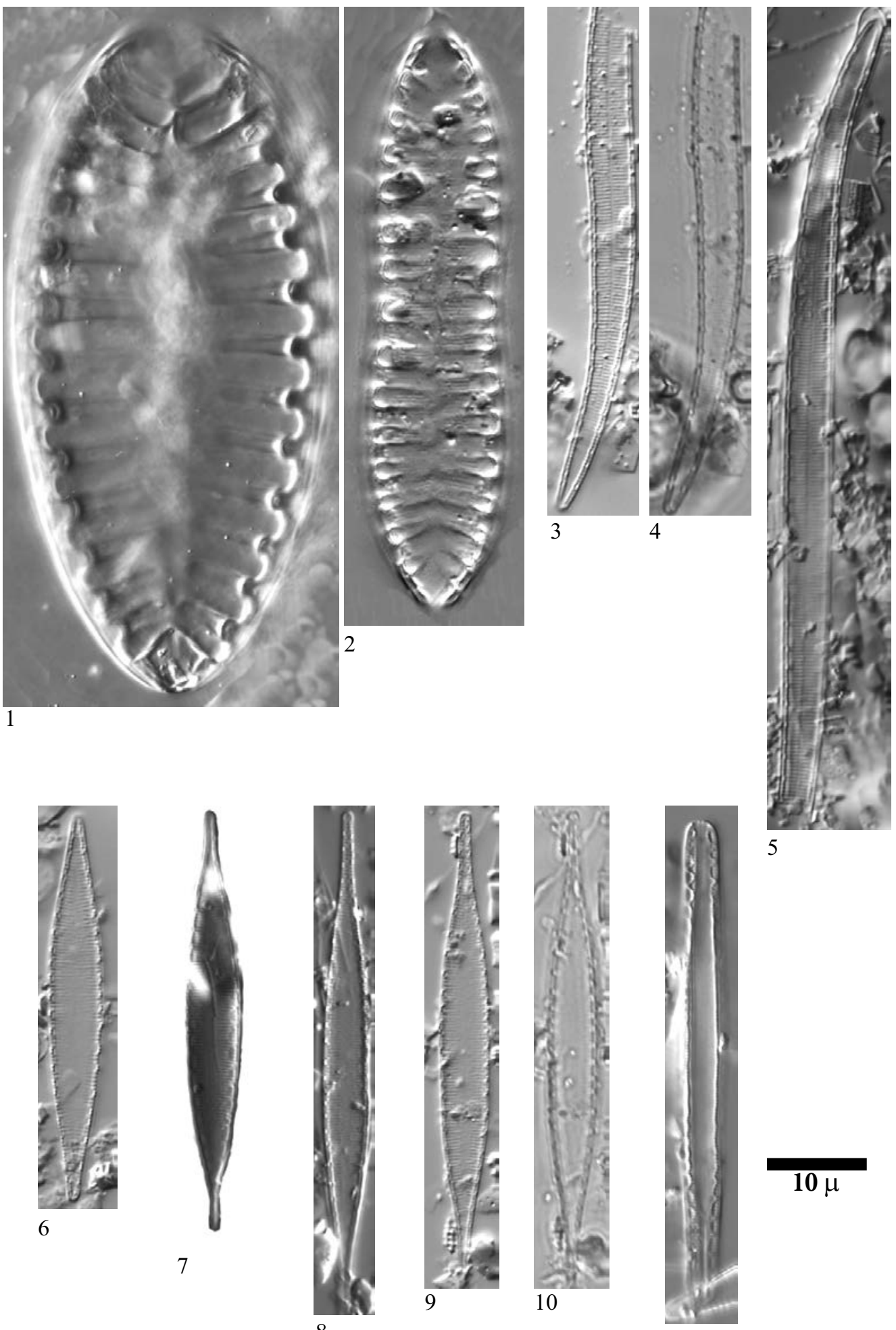

5

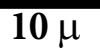


Plate $127 \quad$ LM: x900

SEM: Fig. 1 x900, Fig. 3 x4500

Figs. 1-3 Cymatopleura elliptica (Brebisson) Smith

Figs. 1, 3 Lake Laurenti, sediment PYR111

Fig. 2 Lake Col d'Arretille, sediment PYR12 

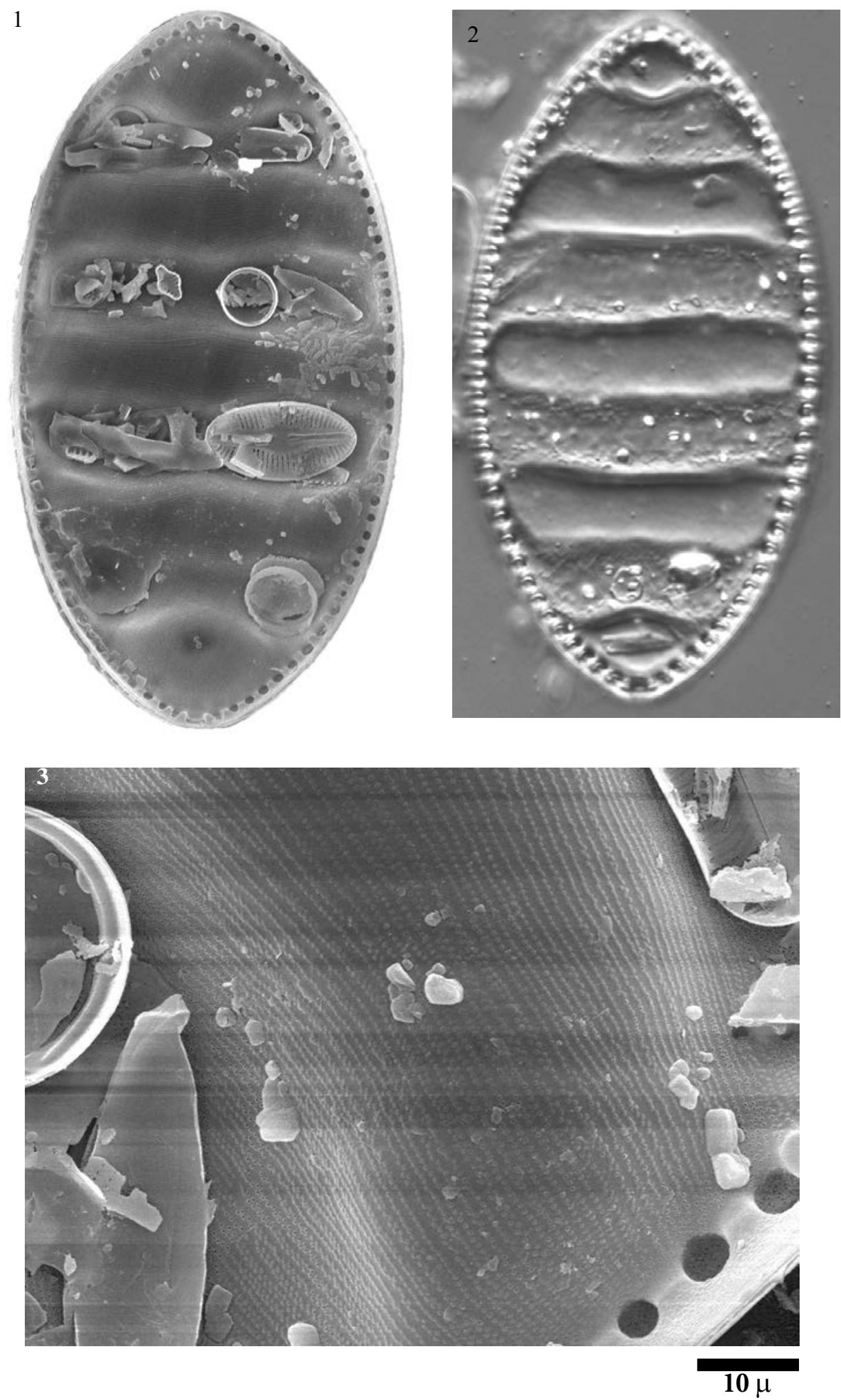

Limnetica, 36 (1): 127-395 (2017) 